\title{
Sketching for Conceptual Design
}

\author{
Empirical Results and Future Tools
}

\author{
Martin Walter Pache \\ Vollständiger Abdruck der von der Fakultät für Maschinenwesen \\ der Technischen Universität München \\ zur Erlangung des akademischen Grades eines \\ Doktor-Ingenieurs \\ genehmigten Dissertation.
}

Vorsitzender:

Univ.-Prof. Dr.-Ing. Hartmut Hoffmann

Prüfer der Dissertation: $\quad$ 1. Univ.-Prof. Dr.-Ing. Udo Lindemann

2. Univ.-Prof. Dr. rer. nat. habil. Winfried Hacker

Die Dissertation wurde am 30.09.2004 bei der Technischen Universität München eingereicht und durch die Fakultät für Maschinenwesen

am 20.01.2005 angenommen. 
This book is available at Verlag Dr. Hut, München, www.dr.hut-verlag.de (ISBN 3-89963-176-5) 
And when you draw a line, it goes where you want.

John Frusciante (2001)

This book is available at Verlag Dr. Hut, München, www.dr.hut-verlag.de (ISBN 3-89963-176-5) 
This book is available at Verlag Dr. Hut, München, www.dr.hut-verlag.de (ISBN 3-89963-176-5) 


\section{Preface of the Editor}

\section{Problem}

Today's companies are exposed to competition in a global market and ever rising expectations of customers. These circumstances compel companies to be innovative and - at the same time - to minimize the time to market.

Digital design tools seem to provide the opportunity to realize ever shorter product cycles. The CAD model may be regarded as the center of a digital design environment. The evolving model can be managed by PDM-systems and handed over to various design and production processes. Thereby, it allows for the development of highly complex products in concurrent and distributed design processes. Yet, these circumstances do not necessarily enable innovation. Innovation requires new ideas and the development of ideas demands creativity.

The most common design tool for that purpose eludes from the digital design environment: the paper sketch. The sketch is probably the most powerful design medium for the development of conceptual design ideas. Yet, there had not been enough knowledge about its mode of functioning in order to develop a digital counterpart of that design medium. While today's CAD-systems are further developed, the gap between the paper sketch and CAD is getting wider and wider.

\section{Objectives}

The overall goal of this thesis is to provide comprehensive insight into the complex processes of creativity that take place when sketches are used for conceptual design. From this insight, the basic characteristics of sketching media emerge, so new sketching tools, which are both effective and efficient, can be developed.

For this purpose, the use of different design media in industry is investigated by the help of two surveys. The sketch, which has been indicated as being most helpful for idea development, is investigated in great detail by the help of 120 design experiments. On the basis of the experimental results, a comprehensive framework for regarding the process of sketching is developed. Moreover, the requirements are formulated, which need to be met by a future medium for conceptual design. The 3D-sketcher, which is the prototype of a digital sketching tool, shows that it is possible to meet these requirements in one new medium.

\section{Results}

The surveys among designers have shown that CAD and sketches are both integral parts of design. Sketches mainly serve for idea development, while CAD is rather used for concretion, verification and documentation purposes. Hence, the impact from CAD on the efficiency and effectiveness of conceptual design is rated lower than the impact from sketches.

The experimental study has revealed that conceptual design sketches may contain elements on various levels of abstraction. Those different levels of abstraction may be mixed up and 
combined within a single sketch. Moreover, the elements within a sketch show linguistic characteristics, so they may be compared to words in a sentence. The relations between the elements in a design sketch bring about highly complex technical "stories," that even pose questions or represent irrational statements. Visual perception and the sequential character of depiction may cause the reinterpretation of those "stories," so the designer may discover completely new concepts within his own sketch. The efficiency of the sketching process is increased by the way that the pencil-tip is moved through the sketch, especially when the designer is not actually drawing any line.

In order to integrate all these findings into one consistent framework, the sketching process needs to be regarded as the designer's communication with himself. That framework is used to elaborate those characteristics of the paper sketch that make it such a powerful tool for conceptual design. A new sketching medium needs to meet certain requirements in order to be an adequate equivalent for paper sketches. Still, such a medium is also constrained by the digital design environment.

The 3D-sketcher is the prototype of a digital sketching tool that has been developed at the Institute of Product Development at the Technical University in Munich. It features a very simple functional principle that meets the requirements mentioned afore. By the help of virtual reality, this device allows for the creation of a conceptual sketch that is truly spatial. A desktop-based version of the sketcher is suitable for a usual office surrounding. Still, when used with different hardware configurations, this medium may be applied to other purposes and disciplines.

\section{Consequences for industrial practice}

Designers, design managers and CAx-managers may benchmark their design environment by the help of the survey results on the industrial use of design media and tools. The designers critically evaluate their actual CAx-tools and make demands on future tools. Softwaredevelopers should carefully consider these statements when they intend to improve actual CAD-systems or develop new design tools. The elaboration of the functional principle of conceptual design sketches should inspire designers to reflect and improve their own sketching techniques. Moreover, the demonstration of the effectiveness of sketches should encourage designers to use this medium until there is a comprehensive equivalent at hand.

The 3D-sketcher is a medium that is capable of replacing the paper sketch sooner or later. As a stand-alone system, the sketcher could be used in a productive manner in the near future. Ideally, the functional principle of the sketcher is integrated into future CAD-system, so sketches can be used at any step of the design process, right within the CAD-model. Workshops with designers, design manager, CAx-managers and software developers are actually carried out at the Technical University in Munich in order to discuss all the matters mentioned afore. 


\section{Consequences for research and science}

This thesis presents numerous scientific results that may inspire other researchers to further investigate sketching for conceptual design. The adaptability of these results on other design disciplines, as well as on other sketching purposes may be validated and specified. The comprehensive framework on sketching as the designer's communication with himself may be used to systematically develop hypotheses, design experimental setting and develop new sketching tools.

The 3D-sketcher needs to be analyzed and evaluated as a medium for conceptual design. On the basis on such a consideration, the sketcher may be further developed. Even the actual prototype provides numerous factors that may be varied in order to optimize its usability. The integration of the sketcher into actual design environments demands the development of a suitable methodical approach. The integration of a digital sketch into the overall flow of design information requires the creation of applicable data formats and interfaces. At the date of publication of this thesis, there are ongoing research projects at the Technical University in Munich that have emerged from the work that is presented in the following.

April 2005

Prof. Dr.-Ing. Udo Lindemann

Lehrstuhl für Produktentwicklung

(Institute of Product Development)

Technische Universität München 
This book is available at Verlag Dr. Hut, München, www.dr.hut-verlag.de (ISBN 3-89963-176-5) 


\section{Acknowledgements}

This Dissertation was submitted to the Technische Universität München in September 2004. It is the result of research work completed at the Institute of Product Development between July 1998 and June 2003.

First of all, I would like to thank my doctoral advisor Prof. Udo Lindemann for his great encouragement and his interest in my work. He allowed me great freedom in following up all my ideas. Due to his style of leadership, there was a great atmosphere of creativity and team work at the institute. Prof. Winfried Hacker, my second examiner, also contributed to this dissertation to a great extent. Throughout four years of interdisciplinary collaboration, he provided me with a fascinating insight into psychology. He always condoned my bold approaches to this complex domain and I thank him for supporting me with his unique combination of expertise and ingenuity. I also want to thank Prof. Hartmut Hoffmann for being the chairperson of the examination committee.

There are many other scientists in the field of empirical design research, who contributed to this dissertation with comments and discussions. Especially the Bamberg project meeting group provided me with lots of inspiration. Very special thanks go to Prof. Petra BadkeSchaub, who introduced me into that group. She has accompanied my occupational development since my studies and has always been the most helpful advisor. Prof. Dietrich Dörner always motivated me with his feedback on my presentations and so did Prof. Herbert Birkhofer and Prof. Gerhard Pahl. Especially Prof. Klaus Ehrlenspiel motivated me with his great interest in my research on creativity and sketching. I enjoyed the lively discussions that we had on our train trips to Bamberg. In his compelling manner, Prof. Ehrlenspiel even involved numerous other travel passengers into the conversation.

I am indebted to my project partner Anne Römer for the most valuable cooperation. I really appreciate her diligence and I thank her for the patience that she exercised with me and my work style.

My very special thanks go to my colleagues at the Institute of Product Development. They provided me with support, critical feedback, ideas, comradeship, and even friendship. I will never forget the good times we had at the ICED's, in Dallas, Hannover, and Coventry, at the Oktoberfest, Nockherberg, and Dienstsport, and in the Keller. Representatively for all colleagues, I want to thank Udo, Bernhard, Heiko, Joachim, Ralf, Ludwig, Gert, Manuel, Heiner, Hans, Bernd, Jens, Christoph, Markus, Ulrike, Marc, Peter, Helmut, Rainer, and Nic.

I thank all the designers and students who participated in the study. They allowed me to have an intimate look at the way they think and act. I also thank the student assistants Holger Emberger, Iassen Raykov, and Maik Maurer, who contributed to the research project and I thank Esther Bley, who proof-read my thesis.

I thank all my friends, who helped me through some hard times while writing this thesis. In this regard, special thanks go to the Meinecke family. Last but not least, I thank my parents, who enabled me to write this thesis in the first place. 
This book is available at Verlag Dr. Hut, München, www.dr.hut-verlag.de (ISBN 3-89963-176-5) 


\section{CONTENTS}

1 An Introduction to Sketching in Mechanical Engineering...................................... 1

2 Conceptual Design in Mechanical Engineering ..................................................... 5

2.1 Sequences of Conceptual Design Work within the Product Development Process .. 6

2.2 Design as a Process of Thought and Action ................................................. 9

2.2.1 Basic Prerequisites of the Human Mind ....................................................... 10

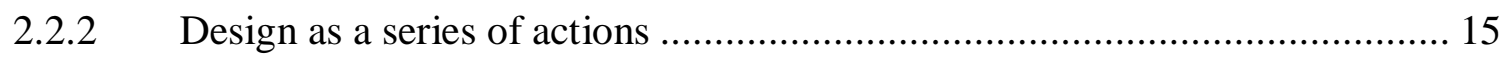

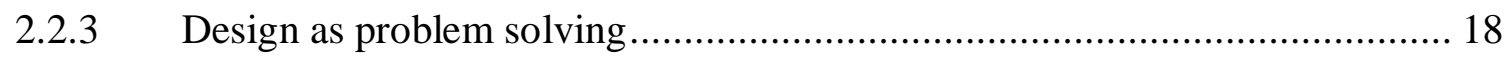

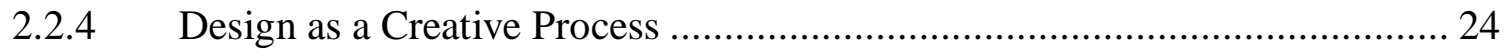

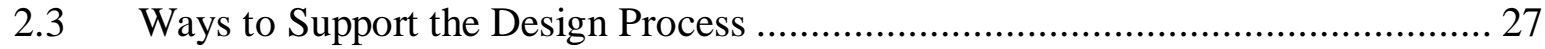

3 Media and Tools for Conceptual Design in Mechanical Engineering ........................... 33

3.1 Illustration of Common Media and Tools for Design ....................................... 34

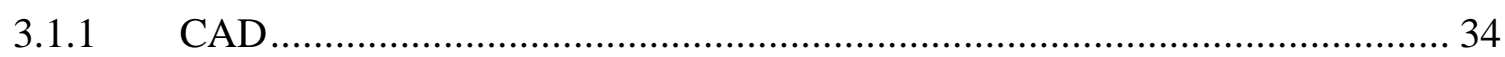

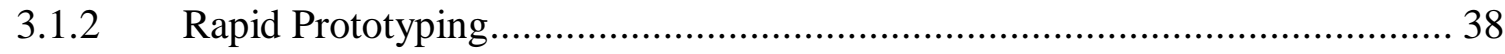

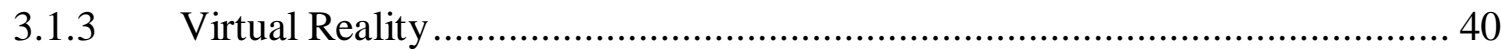

3.2 Actual Use of Media and Tools for Design in Mechanical Engineering ............... 45

3.2.1 Characteristics of Design Tasks in Mechanical Engineering ........................ 46

3.2.2 Use of Common Media and Tools in Mechanical Engineering ...................... 50

3.2.3 Specific Application of CAD and Sketches to Conceptual Design ............... 52

3.2.4 Benefits from the Application of CAD and Sketches ................................. 57

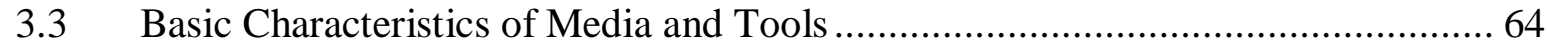

3.3.1 A Model for Soliloquy using Media as Communication Channel .................. 64

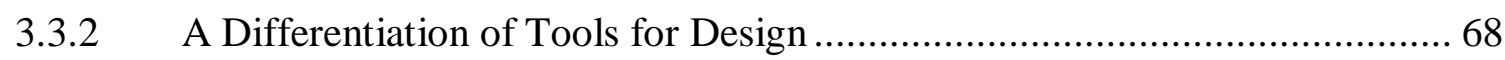

4 Sketching - A Major Element of Conceptual Design............................................. 71

4.1 An Experimental Investigation on Design Sketching ..................................... 71

4.2 The General Characteristics of a Sketching Process in Conceptual Design ........... 78

4.3 Depiction Modalities and Information Categories in Sketches ............................. 83

4.4 Perceiving Conceptual Design Sketches ...................................................... 95

4.5 The Meaning of the Pencil's Motion in Sketching ........................................ 102

4.6 A General View on the Sketching Process .................................................... 116 


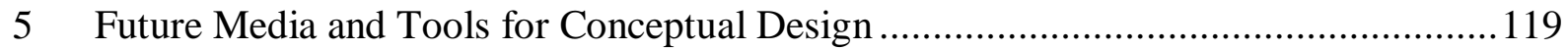

5.1 CAx - A Necessity in Product Development ..................................................... 119

5.2 Characteristics of Future Sketching Media ..................................................... 123

5.3 The 3D-Sketcher - An Example of a Digital Medium for Conceptual Design ..... 125

5.3.1 Sketching Lines in Space - The Basic Idea............................................ 125

5.3.2 Further Functionalities and Features of the 3D-Sketcher.............................131

5.3.3 Different Hardware Configuration of the 3D-Sketcher.............................. 133

5.3.4 Integrating the 3D-Sketcher in a CAx-Environment for Engineering Design134

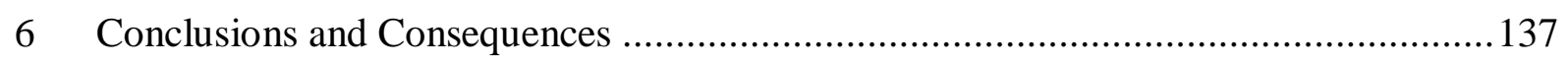

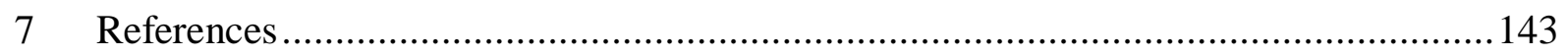

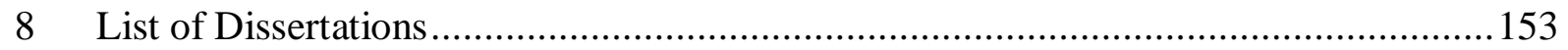




\section{An Introduction to Sketching in Mechanical Engineering}

At the Technical University of Munich, a workshop was held that aimed to bring together designers from industry and developers of design software. At that workshop, a designer, who worked at an automotive supplier for clutches, stated that he would like to be more creative in his design work. Yet, he complained that the design software restricted him in doing so. On this complaint, the representative of a company that distributed one of the major software packages responded that "for a supplier, the restrictions from the automotive manufacturer are so severe, that there is no room for creativity anyway." This reply may reveal a potential major misunderstanding of creativity. This designer may not be designated to invent a new functional principle for clutches, but does he therefore not need to be creative?

In mechanical engineering, creativity does not only take place in design from scratch and a creative solution does not necessarily need to include some revolutionary new functional principle. Strong restrictions may exclude the development of new functional principle and may necessitate the reuse of a highly optimized working principle. The adaptation of a given main solution may still require new concepts for subsidiary systems and therefore demand creativity in particular. Conceptual design does not need to occur only when it has been planned to occur. At the very least, methods and tools for design should not distinctively preclude the emergence of new conceptual ideas.

The designer's statement quoted above also reveals the dissatisfaction of designers with their design tools' support of creativity. Innovation has always been a driver for technological, economical and social progress. Computer-aided design tools have surely paved the way for the development of today's products; yet, they do not create the corresponding ideas. At the time being, it is still the designer, who comes up with new ideas and who has done so at all times - even before computers were invented. But there is another design tool, which has been in use for a much longer time and still serves as a powerful design aid (as we will see in the course of this thesis): it is the ordinary paper sketch.

A sketch is available almost everywhere at any time- even when it is actually just a napkin or a beer coaster. Researchers who investigate design disciplines other than mechanical engineering (such as architecture $^{1}$ ) often emphasize the benefit from sketches for creative design. This tool supports the occurrence of design ideas in manifold ways, although it is a rather simple device itself.

But what is the state of affairs in the case of mechanical engineering? Should the dissatisfied clutch designer use sketches in order to be more creative? And if he still needs to use computer-based design tools (which is likely to be the case), then how should sketches interact with those tools? Let us consider one finding from the following thesis beforehand: Sketches and computer-based tools both play important roles in today's design processes in

\footnotetext{
${ }^{1}$ see numerous contributions to the conference "Visual and Spatial Reasoning in Design" (GERO \& TVERSKY, 1999).
} 
mechanical engineering. However, there is no sufficient methodological and technological framework that integrates both these design tools in such processes.

What needs to be clarified, is the particular benefit that may be derived from the use of sketches in mechanical engineering. Moreover, the mechanisms of problem solving that are facilitated or even enabled by the sketch need to be revealed and understood. On basis of such an insight, those characteristics, which actually determine effectiveness and efficiency of a sketch as a design tool, may be identified. Only then may sketches best be integrated into today's and tomorrow's design environments. The development of a new type of digital design device that combines the advantages from paper-sketches and computer-based tools in one single medium would be possible. In the following course of this thesis, we will regard findings from a four-year research project that has dealt with all these subjects.

The project was a cooperative effort between researchers from the areas of mechanical engineering $^{1}$ and psychology ${ }^{2}$ studying future tools and media for conceptual design ${ }^{3}$. The researchers carried out two surveys, several workshops and more than 120 experiments with students and designers. Based on the results from these activities, the prototype of a new type of future design tool was set up. The so called "3D-sketcher" allows for the creation of "truly" spatial sketches in a completely intuitive way with the help of Virtual-Reality-technology.

In the second chapter, we will explore some scientific background from both the areas of design and psychology. At first, we will clarify the term "conceptual design" in general (as far as possible and necessary). Starting from a look at the human mind from the angle of cognitive psychology, we will explore design as a series of actions, as problem solving and as an application of creativity. Against this background, we will regard the support for design processes that is offered from engineering design methodology.

The third chapter provides a closer look at common media and tools for conceptual design in mechanical engineering. A survey is presented that highlights the way in which those media are applied by designers from industry. It elaborates the benefits and drawbacks from the use of sketches and computer-based tools, as well as the designer's claims against future design media. Based on these results and on some theoretical consideration, we will get to know a scientific framework that will help us to fit together the experimental findings from the next chapter.

The results from an extensive experimental study on the use of sketches in mechanical engineering are presented in chapter four. We will start from statistical analyses that examine commonalities and differences between the sketching behaviors of 61 designers and students, respectively. Based on these results, we will choose some very characteristic experiments for further analysis by means of case studies. While viewing vivid design sequences and actual design sketches, we will go further and further into the details of creative conceptual design with the help of sketches. We will gain insights in various ways to depict abstract and even

\footnotetext{
${ }^{1}$ Institute of Product Development, Mechanical Engineering, Technical University of Munich

${ }^{2}$ Workgroup „Knowing - Thinking - Acting“, General Psychology, Technical University of Dresden

${ }^{3}$ The project was funded by the German Research Foundation (Deutsche Forschungsgemeinschaft)
} 
irrational information entities and how these entities are arranged in sketches like the words of a "graphical language." The perception and interpretation of design sketches and the way these processes may lead to the (unintentional!) occurrence of new conceptual ideas will be a subject matter. Even a single motion of the pencil, which spans only a fraction of a second, contributes to the efficiency of the use of sketches for conceptual design. In summary, numerous different aspects of sketching can be identified that interact and make up this unique design medium.

As a consequence, we will get to know the concept of the "3D-sketcher" in chapter five. This future design medium aims to comprise the characteristics of classical sketches and the advantages from digital environments. This medium provides very intuitive creation and handling of sketches with the help of Virtual Reality. Such 3D-sketches provide completely new ways of representing any kind of spatial abstract information. Considering the actual prototype of the 3D-sketcher as just one example, we will consider other possibilities to configure such a medium. Thereby, we will explore several different applications of the sketcher in mechanical engineering as well as in other design-related and even artistic disciplines.

After having gone through this thesis, we may not be able to answer all the questions that we raised some paragraphs before with one hundred percent confidence. However, this is not the goal of this thesis. It is probably neither possible to treat such an extensive subject within one thesis, nor possible to achieve necessary results in one research project. In the first place, the objective of this thesis is to open up a comprehensive framework that allows for correlating all the different factors involved in sketching. That framework may be used for discussion, reasoning and forming one's own mind about this interesting subject. Thus, it may help other researchers to generate hypotheses and set up research projects about sketching, creativity and conceptual design. Nevertheless, this framework may help students and professional designers to reflect upon their own procedures in order to work more creatively and efficiently.

Beyond this framework, this thesis provides numerous well-founded hypotheses about sketching in design that could not be verified within this project. These hypotheses are open for verification, modification and even refutation by anyone. Yet, there are also a number of scientific findings that were gained, verified and which are presented in depth in this thesis. These results may well be used within other research projects that deal with subjects that are related to sketching and creativity. They may also be used by the developers of soft- and hardware for design in order to ensure the applicability, effectiveness and efficiency of their future products. The presentation of the 3D-sketcher and its functional principle especially aims in that direction. Again, these results may be of great interest for designers and designers-to-be in order to better understand those parts of their challenging profession that elude from conscious perception.

Lastly, this thesis provides a number of vivid examples of real sketching processes that may even arouse interest in a reader who is not directly related to this interesting topic. 
This book is available at Verlag Dr. Hut, München, www.dr.hut-verlag.de (ISBN 3-89963-176-5) 


\section{Conceptual Design in Mechanical Engineering}

This thesis will mainly deal with processes, media and tools for conceptual design in mechanical engineering. In order to go into the author's ideas on this subject, it is essential for the reader to share a common understanding or at least a common definition about the term "conceptual design" with the author. So what is conceptual design? Viewing existent definitions, it shows that it is even hard to say what conceptual design is in general. It may either be a phase of the design process, the procedure of developing a certain kind of product definition or a certain kind of product definition itself (see for example PAHL \& BEITZ 1996). To gain a first insight to "conceptual design", one might split this term into its components, which are concept and design.

In this context, design seems to be the more comprehensive and basic term, so we consider its definition at first. According to MILLER (1996), "design is the thought process comprising the creation of an entity." This definition points out several important characteristics of design still, we will not adopt every one of them as the basis of our further consideration. In this definition, "process" probably is the most essential term, because it shows what Miller considers design to be in general: it is a series of actions, changes or functions bringing about a result. We will not determine whether design is only a process of thought or also includes essential processes of act, which not just supplement or support processes of thought. In the following considerations, all (mental and actual) sub-processes within a design process will be taken as being a part of design. Furthermore, this definition names what happens when a process of design takes place: a - not further specified - entity is being created, which means it is brought into existence and therefore has not existed before. Miller indicates an entity as the "thing" being created and thereby leaves open what category of "thing" emerges from a design process.

In case of a conceptual design process, exactly this "thing" is specified by the term concept. Following the dictionary, a concept can be an idea, a plan, or a scheme (MERRIAM-WEBSTER 2003). In mechanical engineering, such a concept is not just a general idea such as "Let's build a car, how about that?". PAHL \& BEITZ (1996) specify a concept as a principle solution for a product based on working principles and working structures. It may have been generated out of functional considerations that have been undertaken beforehand and may afterwards result into a constructional definition. Figure 2-1 shows the functions structure, the working structure and the construction structure of a clutch. The working structure exemplifies the conceptual stage of the design. But methodology does not only describe the elements and the structure of a concept; it also points out at what time a concept emerges. Prescriptive schemes for design procedure, such as those suggested by industrial standards and norms (see for example VDI-RICHTLINIE 2221, 1993), usually state that creating a concept should be one of the early steps within design procedure. In conjunction with the definition from dictionary, a concept in mechanical engineering may be seen as an idea for the later product and also as a plan for further complementation of the ongoing design process. So, a concept is a preliminary stage of the later, final product description that concludes the design process. 


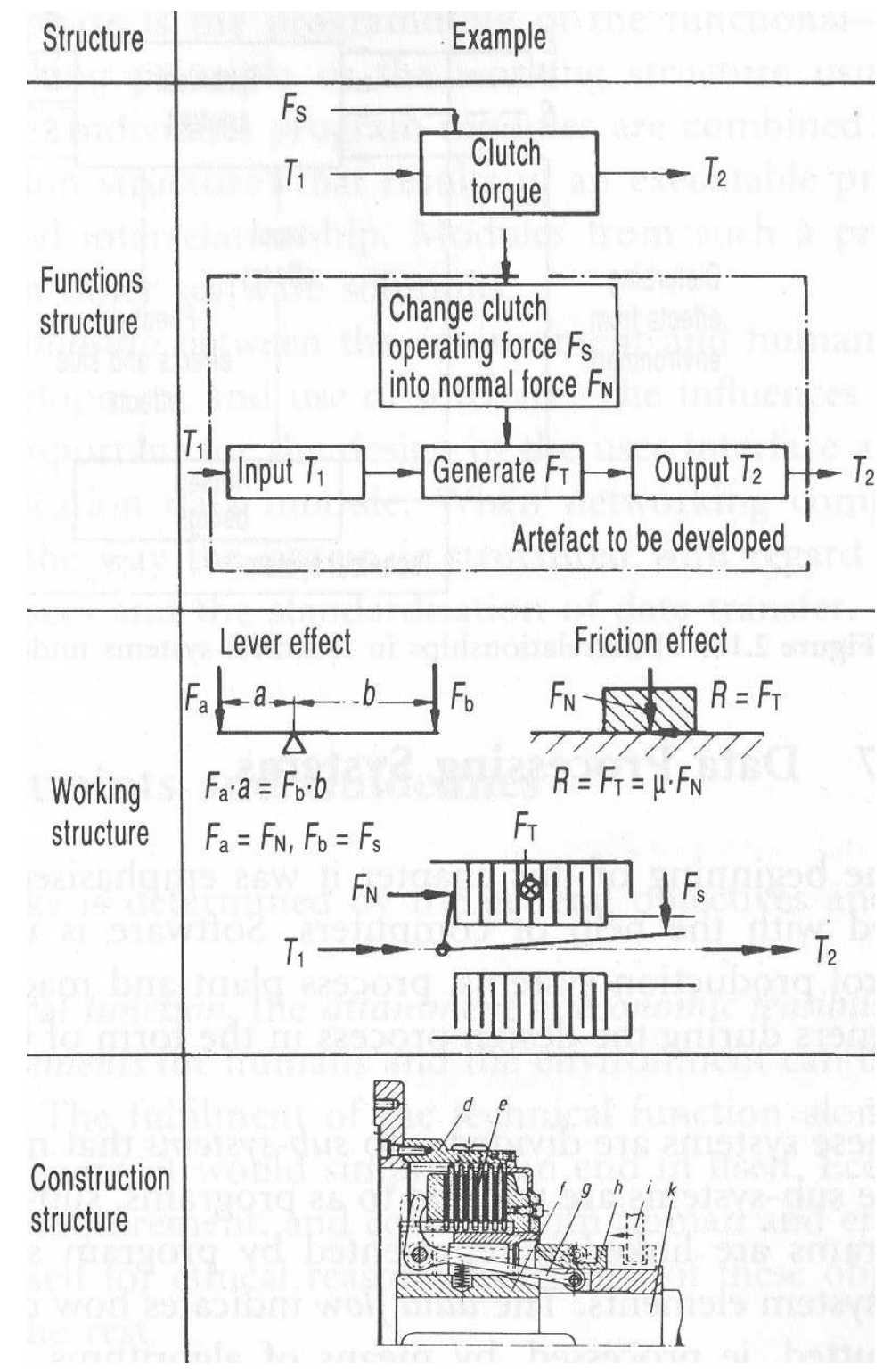

Figure 2-1: Functions structure, working structure and construction structure of a clutch (PAHL \& BEITZ 1996, p. 43)

\subsection{Sequences of Conceptual Design Work within the Product Development Process}

If a product description is preliminary, what does this mean for its elements and structure? Generally spoken, there are - at least - three ways in which a product description can be preliminary. At first, it can be incomplete, which means that essential parts of the description are not defined, so elements are actually missing. Still, even if the description of some future 
product is completed, it may be preliminary. And that is, secondly, if it is false or irrational. It may be false according to the demands made on the future product or may be irrational in itself if elements contradict each other and if the structure is inconsistent. Thirdly, a product description is preliminary if it is more abstract than the final description. The process of abstraction is to omit certain features of an object. DÖRNER (1987, p. 18) defines abstraction (similar to the definitions used in engineering design research) as "omitting inessential attributes respectively accentuating essential attributes". An abstract entity summarizes all (more concrete) entities that have those features in common which are not left blank in the abstract entity. The other features may have any specific value. It is essential to carefully differentiate abstract from incomplete since both these information modalities may appear to be similar although they are not (ARNHEIM 1969, pp. 98). An abstract description may contain all necessary elements, but they are not defined entirely. So, an abstract element within a product description represents a variety of more concrete elements, which may be part of the final description, yet it leaves open which one of them it is going to be. This decision still has to be made and therefore the product description is preliminary.

All these potential characteristics of a preliminary product definition hold for a concept in engineering design. We will thoroughly address incompleteness and irrationality later, since these characteristics may be important stimuli for creativity. Still, the most important characteristic of a concept probably is abstractness, since it enables an essential process of solution development: By using abstractness, a designer can define a limited number of solution variants and depict his choice using only one representational element. Additionally - and this is even more important - he only needs to remember one piece of (abstract) information that represents a variety of concrete elements. Thereby, the designer can stepwise confine a huge number of possible solution variants to reach the final product definition. And this shows the importance of decisions made on an abstract level: limitations made to the solution space that are constituted by abstract predefinitions elude other possible solutions from further consideration within the latter design process and design freedom is reduced (UlLMANN 1997). The more abstract the predefinition is, the less the properties of the future concrete solution possibilities may be estimated (BERNARD 1999, pp. 28). Likewise, the more difficult it is to say whether a good solution was just being excluded. The further the design process goes on, the higher the effort to reparate wrong decisions will become (EHRLENSPIEL et al. 1998, pp. 10). So, all decisions made on an abstract, conceptual level are potentially essential for the result and therefore for the success of a design process. So the importance of conceptual design should not be underestimated.

Guidelines for design often suggest the definition of the entire future system on a conceptual level before proceeding to a more concrete level. Consequently, the phase of conceptual design has become a commonly used term when design processes are described (PAHL \& BEITZ 1995). Although the iterative nature of those guidelines is emphasized, one might assume that in real design processes a comprehensive conceptual design phase is always performed. However, this is not compulsory and observations have shown a variety of possible procedures (GÜNTHER 1998, VON DER WETH 2001). Consequently, such procedure schemes are considered to be too strict and rigid (EHRLENSPIEL 2003, p. 296). Deliberately, no such conceptual design phase is considered within this thesis. Observations and considerations made within this thesis rather refer to conceptual design sequences, which do 
not need to have a distinct duration and may appear at any point of time within the overall design process. Regarding concept development as a comprehensive phase that is finished by a milestone may be helpful, yet necessary for process management. Still, methods and tools for conceptual design cover every conceptual design sequence, and must be available whenever it happens.

In the run of this thesis, we will consider conceptual design from two very basic points of view. At first, we will follow the tradition of design methodology research and look upon conceptual design as a process of information processing. Hence, we will apply theories from cognitive psychology, assuming that essential parts of design may be seen as design problem solving.

Let us briefly discuss this approach: According to DöRNER (1987, pp. 10), a problem is given if there is an undesired actual state and barriers of any kind, which (at least in the beginning) prevent the transformation of the actual state into a desired goal state. He describes two variables that determine the nature of a problem. These are:

- the degree of clarity of the goal state and

- the degree of awareness of possible means.

When a design task starts, there is usually a list of requirements given, which states the desired behavior of a technical object. Still, this information does not reveal the structure of the future technical object, which would be a clear definition of the goal state. Defining the goal state properly is part of the design problem, as well as getting to this goal state. Therefore, design is problem solving with a low clarity of the goal state and design problems may be called ill defined problems.

Additionally, not all possible means are aware to the designer at all times during design. Let us consider a designer who is sketching in order to develop a concept. One might say that this designer is very well aware of every action he is capable of, since this is basically moving the pencil in every direction within $360^{\circ}$. By these means he can sketch every kind of shape or symbols and thereby represent every possible technical element. This "awareness of all possible means" is only true for the most basic level of action. Conceptual design can be seen as finding the right combination of technical elements to achieve a certain behavior of the overall working structure. The number of known technical elements is certainly huge and there is probably an infinite number of combinations that alter the behavior of the system they are added to. In theory, the designer would need to be able to overview all possible combinations, but this is impossible due to limitations of human memory (SACHSE \& HACKER, 1997).

The definition of design from Miller that we adapted before emphasizes the process of creation. Therefore, we will also consider conceptual design as an application of creativity. Although creative processes may be seen as being "only" one special case of problem solving, this point of view provides us with some very interesting effects, such as the sudden idea.

Yet, designers in mechanical engineering can hardly be seen independently from their industrial context. Design is a set of goal-oriented working tasks and requires specific and complex processes of action to ensure the final realization of the technical system being 
designed. Design involves essential components of external psychomotor activity, such as sketching, typing, mouse-clicking and speaking. Therefore, our second point of view on design will be from the prospective of action theory. HACKER et al. (2003) proposes a framework for research in engineering design in terms of a complex working activity. $\mathrm{He}$ claims the consequent application of action theory in the European tradition of such researchers as LEWIN (1926) and LEONTJEW (1979). With the help of this approach, influences from numerous working tools, methods and procedures on the design process can be regarded. The author carried out many workshops with designers from industry and it has shown that a number of designers feel strong restrictions and biases being exerted upon them from CAD-systems (VDI-NACHRICHTEN 2001). Theoretical considerations in design research approve this finding (STACEY et al. 1999). Regarding design from a purely cognitive point of view might not cover this effect in a sufficient manner.

In the author's opinion, information processing theory and action theory need to be combined to a rather general framework for research on individual design in mechanical engineering. In engineering design, problem solving needs to result in action that embodies the emerging information and this embodiment again feeds back into the problem solving process. The effect from one of these processes on design cannot be determined without the consideration of the other process. The "gap" between observable design action and idealized algorithms for information processing needs to be closed by a consistent model. Then it may become easier to explore the effect from those numerous influencing factors that seem to make design processes so unpredictable. The individual with its declarative and procedural knowledge forms the background to the cognitive process and the action process, which are both determined by the task, the actual situation, the working environment and so on. Hence, it is so hard to support designers by appropriate tools and methods. For design teams, the approach even needs to be broadened to include theories of social and communicational interaction. In order to improve media and tools for individual design, we will not focus on team design.

In this thesis, the attempt is made to show some aspects of the relation between cognition, action and creativity for conceptual design in mechanical engineering. Through the observation of actions carried out by sketches and through the analysis of the change of information represented in the sketch, a fundamental and close interlocking between thought and action in design is demonstrated.

\subsection{Design as a Process of Thought and Action}

In the last chapter, we have discussed what conceptual design is. Now we will have a closer look on how the design process in general may be carried out and what may be the special characteristics of conceptual design sequences in particular.

The way design processes are carried out is strongly dependent on the media through which they take place. Starting from the mind as the medium and container of mental processes, we will explore other representational media in a general way. Against this background, we will then look upon the design process as a series of actions, as a problem solving process and as an application of creativity. 


\subsubsection{Basic Prerequisites of the Human Mind}

The human mind or memory respectively, in which information of any kind is stored and processed, may be viewed from numerous psychological and medical angles. In this case, we will mainly draw upon models from cognitive psychology, which provide a very functional point of view for our purpose.

At first, we will regard the functional structure of the human memory. ATKINSON \& SHIFFRIN (1968) propose a model to describe human memory, which names different areas of data storage that fulfill different purposes in information processing (see top of Figure 2-2). At first, there is the sensory register which receives information coming from the sense organs. The register holds this information for a fraction of a second and provides it for the other memory areas to be taken up. The short-term memory accesses the sensory register and stores those information entities that are important for further processing. As long as these entities are within the short-term memory, the person is aware of the information. The capacity of this memory area is fairly limited and, as the name implies, information is not stored in perpetuity - we will consider the characteristics of this important memory area in detail later on. From short-term memory, information can be transferred to long-term memory, which is comparable to the generally known process of "learning something by heart". Still, certain information can switch over directly from the sensory register to long-term memory without being aware. Long-term memory corresponds to the colloquial meaning of the term "memory". Long-term memory is virtually unlimited (DÖRNER 1987, p. 29), since nobody has yet been able to specify or at least estimate its capacity (HUSSY 1984A, p. 187). Information within this storage is not aware, but it is available for being made aware respectively being remembered.

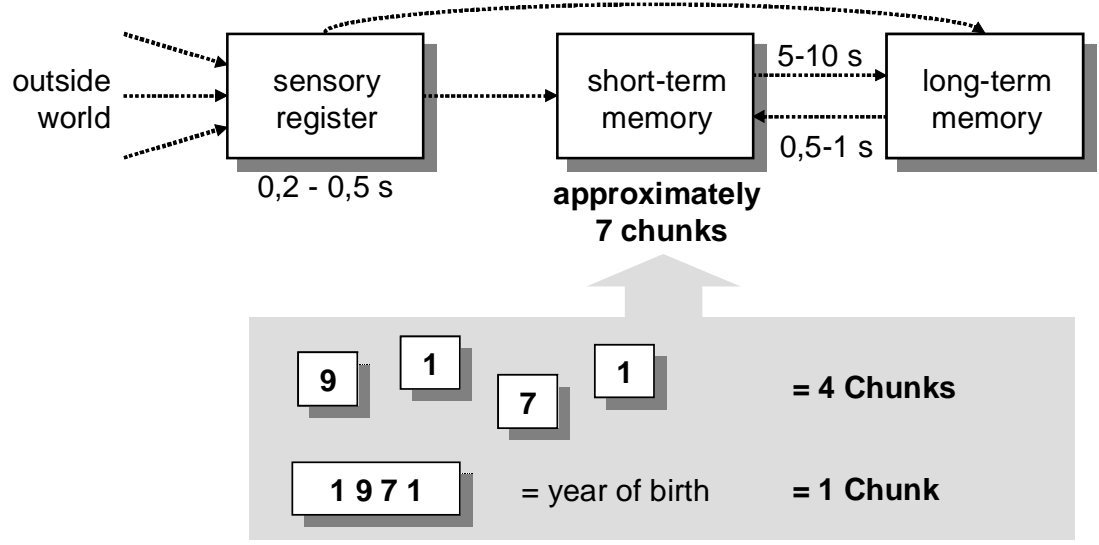

Figure 2-2: Model of human memory (following ATKISON \& SHIFFRIN 1968) and the aggregation of chuncks.

Let us now have a closer look at the way information is processed within short-term memory. It may be supposed that an information processing unit called working memory uses this storage (HUSSY 1983). The term "working memory" implies the importance of its role for 
processes of thought. It is comparable to the working memory known from PCs, since only in this area information can consciously be altered. The mental process that most people would commonly characterize as reasoning, takes place within short-term memory. Although great achievements have been accomplished by human beings due to reasoning, the capacity of short-term memory is surprisingly small. Only about seven (plus/minus two) independent information units, called chunks, can be kept aware simultaneously within the short-term memory (MILLER 1956). Compared to the working memory of today's PCs, this number is frighteningly small, recalling numerous Science Fiction movies that predict the reign of machines over mankind.

Still, the human mind has developed ways to cope with just seven chunks. One chunk may contain much more information than one bit within digital data storage. An example may clarify this: The sequence of numbers 1,9,7 and 1 occupies four chunks if the numbers are kept aware independently. But when the numbers are interrelated, they form the four-digit number 1971, which is the author's year of birth. So for him, this sequence represents just one piece of data and therefore occupies only one chunk (DÖRNER 1999A). The aggregation of several pieces of information into fewer units provides relief of short-term memory (see bottom of Figure 2-2).

Aggregated information units only make sense for reasoning as long as its individual elements are still accessible. The initial elements, as well as their relations among each other, can be stored in the long-term memory. Thereby, the components of an aggregation are still available. Actually, long-term memory especially supports certain kinds of aggregation by its information structure. According to DÖRNER (1987, pp. 27), factual information are stored within the epistemic structure. Information is stored in the form of a semantic net, which means that any information item can have any number of contextual relations to other items (based on CoLLins \& QUILLIAN 1969). The items may be seen as the knots of the net, the relations between the items are represented by the strings between the knots. There are three general kinds of relations between information items that are represented in the epistemic structure (DÖRNER 1987, p. 32):

- part-/whole-relation:

$\mathrm{X}$ “is part of" $\mathrm{Y}$ respectively $\mathrm{Y}$ "consists of" $\mathrm{X}$

- subordination-/superordination-relation:

$\mathrm{X}$ “is a (general kind of)" $\mathrm{Y} \quad$ respectively $\mathrm{Y}$ “is a (specific kind of)" $\mathrm{X}$

- spatial and temporal relations:

$\mathrm{X}$ "follows" $\mathrm{Y}$ respectively $\mathrm{Y}$ "precedes" $\mathrm{X}$

$\mathrm{X}$ "is above" $\mathrm{Y}$ respectively $\mathrm{Y}$ "is underneath" $\mathrm{X}$

Figure 2-3 schematically shows the representation of a technical system as a semantic net that contains part-/whole-relations and subordination-/superordination-relations (spatial and temporal relations are not represented to simplify matters). These relations suggest the way that the previously addressed aggregation of information could work: instead of keeping aware of a lot of single information units, a person thinks of the whole, which is formed by the aggregation of the units. Sometimes, such an aggregation needs to be generated 
beforehand. That is the case if the aggregation has not existed or at least has been unknown to the person before. This new aggregation then needs to be remembered ("saved" to the longterm memory) so it can be split up in turn if necessary. This special process of aggregation which generates a new combination of elements may actually be a very basic process of creativity - we will get back to this subject later on.

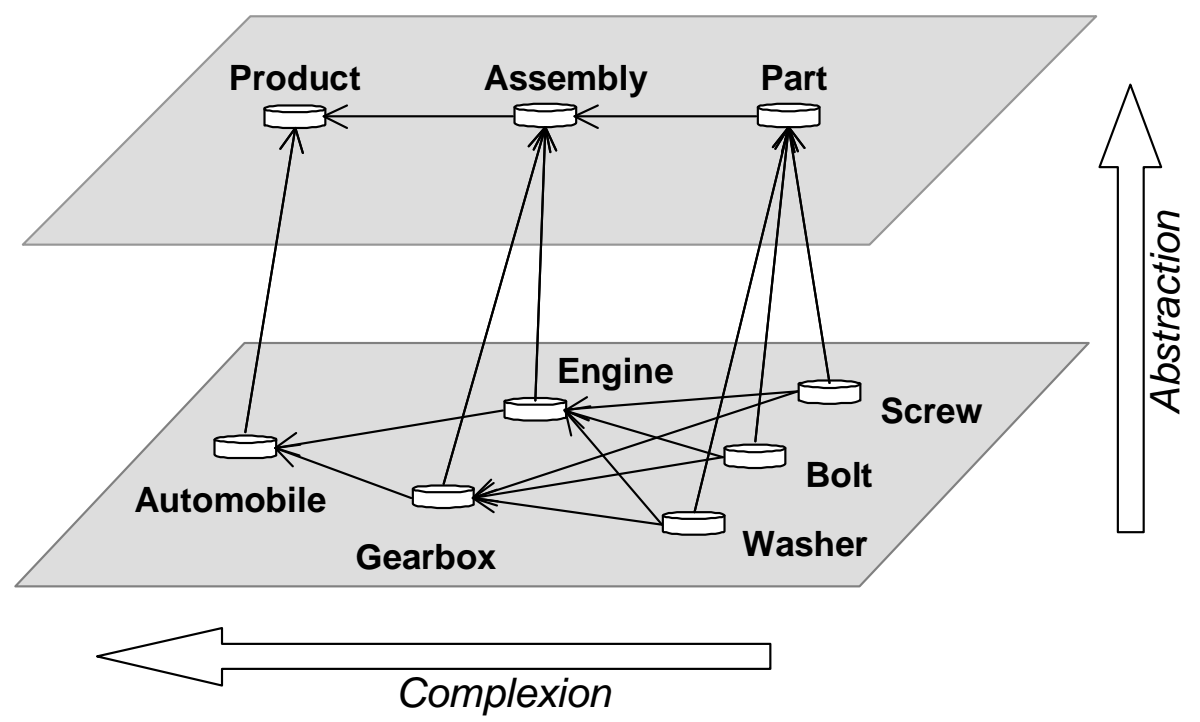

Figure 2-3: Semantic net containing part-/whole-relations and subordination-/superordination-relations (following DÖRNER 1987, p. 32)

More generally considered, the semantic structure lays ground for some basic operations of information processing within short-term memory. Processing a certain information unit may be carried out by scanning all knots in the adjacency of the initial knot and testing them according to certain criteria(s). Another way of processing may be the movement from knot to knot along a certain kind of relation and thereby activating (making aware) all (information) units along the way (DÖRNER 1987, p. 104).

The approach of proposing that the working memory is a system that processes verbal information (as we have implied in the preceding paragraph) is not yet sufficient. A rather similar system is proposed for the processing of visuo-spatial information, which we will refer to as imagery in the following considerations. BADDELEY (1997, pp. 71) calls this instance the visuo-spatial sketchpad. There has been an intense debate over the last years about whether the mental representation of such images should be regarded as being analogous ${ }^{1}$ (as stated by

\footnotetext{
${ }^{1}$ An analogous representation is comparable to usual pictures consisting of pixels. The basic element of an analogous representation is a point with a location and a value. The spatial relations between the elements of the representation correspond to the spatial relations between the actual elements.
} 
KOSSLYN et al. 1979) or propositional ${ }^{1}$ (as stated by PYLYSHYN $1973^{2}$ ). There are several implications on the characteristics of the processing of images from this differentiation. Yet, there seems to be counter-evidence for both approaches, such that mental images seem to contain both propositional and analogous aspects.

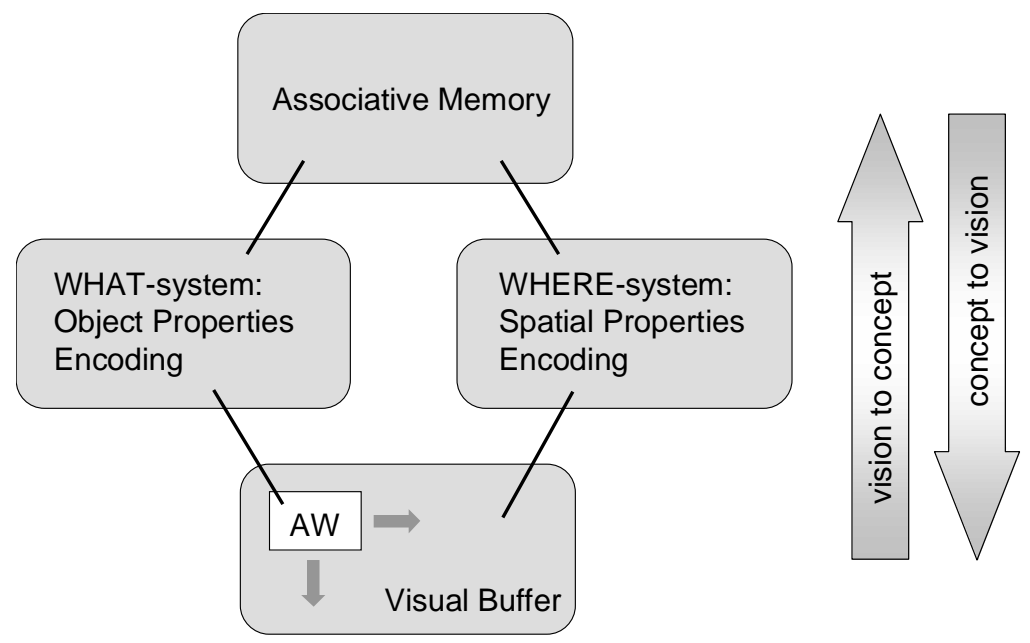

Figure 2-4: Vision system according to KOSSLYN (1994, pp. 70)

In order to gain experience on how a mental medium for visuo-spatial information might be like, we will briefly introduce some possible characteristics derived from KosSLYN (1994, pp. 70). He describes a vision system (see Figure 2-4) that consists of four basic components. The Visual Buffer (VB) stores a mental image and allows for the segregation of some scene into regions associated with different objects. There are two separate processing modules each responsible for what is in an observed scene (WHAT-system) and for where the objects in the scene are (WHERE-system). The VB is scanned by an Attention Window (AW), which permits the system to focus on a single object at a time. The AW provides the interface between the VB and the WHAT-system, which extracts features and categorizes the contents of the AW. This process permits the assignment of noun labels to the objects. By the WHERE-system, output from the VB is assigned a 3D coordinate system (viewer-centered, object-centered or both), and the location, size, and orientation of each object in the scene are

\footnotetext{
${ }^{1}$ A propositional representation is a "mental sentence" that specifies unambiguously the meaning of an assertion (KOSSLYN 1979, p. 5). Propositions have relations that connect entities. Symbols may be seen as the basic element of propositions.

${ }^{2}$ PyLYShyn (1973) disagrees with the idea of depictive representations. He maintains that if mental images were depictive, a 'little man' would be needed to look at the pictures to interpret them in the way that "real" pictures are interpreted when they are perceived (this is the homunculus problem). If a man looks at the pictures and sees and interprets the pictures - how do we interpret what is going on in the little man's head? For this reason, Pylyshyn believes in propositional representations.
} 
extracted. The WHERE-system is responsible for classifying the relations between objects in the scene. The visual system makes contact with non-visual cognition in an Associative Memory (AM). It is in this AM that vision and language come together and both the WHATand WHERE-system play a role in the AM. This process is meant to account for the direction from vision to concepts, but it may run in the opposite direction as well, so imagery can be created from concepts. Hence, the WHAT- and WHERE-system are in turn under the influence of the AM.

KOSSLYN (1994, pp. 74) supposes that the medium for mental imagery

- is spatially limited,

- has an area of highest resolution at its centre and

- features a "granularity" that may blur details.

He claims that mental images are processed in the same way as perceived images with three exceptions:

- Mental images fade rapidly,

- they are created from stored information and

- such images are malleable (they can be rotated, zoomed and altered in many ways).

The long-term memory may serve as a relief for the short-term memory (no matter if it is spatial, visual or conceptual) in terms of an overflow basin. But furthermore, information from long-term memory may be the object of reasoning itself. Still, there is a broad variety of other media that may fulfill these purposes as well: external representational media, such as a scripture or a sketch on a piece of paper for example. Later on, we will go into detail according to the specific characteristics of certain media and their use. But even generally considered, there are factors that fundamentally distinguish different media. Without the claim to be exhaustive, such factors are:

- reliability of storage

(In contrast to the human memory, external media usually do not forget. However, computer-based media sometimes $d o$ forget. Anyone, who has had a system crash yet, knows about that...).

- time for filing (It may be faster to write down a poem than to learn it by heart...)

- information structure

(A written text is a single chain of terms, but some diagrams allow to arrange information units in a net-like structure...)

- capacity

(A sketch is limited by the size of the piece of paper, the capacity of digital media is determined by its hard drive, but there are no limits known for human long-term memory...) 
- time for access

(Seeing a picture happens quicker than reading the verbal description of the same picture...)

Still, the most fundamental difference between internal media and external media probably is the functional principle of filing and accessing information. On the one hand, filing requires certain translational processes to make mental information compatible to a "real" medium's structure. On the other hand (and probably even more fundamental), filing into an external medium requires action, which is observable motor activity that is regulated by very specific, partly unconscious processes.

\subsubsection{Design as a series of actions}

A very useful approach to describe design is to consider it as a complex process of goaloriented actions, using models known from action theory. The most common model for action regulation is probably the TOTE (Test-Operate-Test-Exit) unit that has been introduced by MILLER et al. (1960). Its structure is represented in top of Figure 2-5, exemplified by the action "drawing a square".

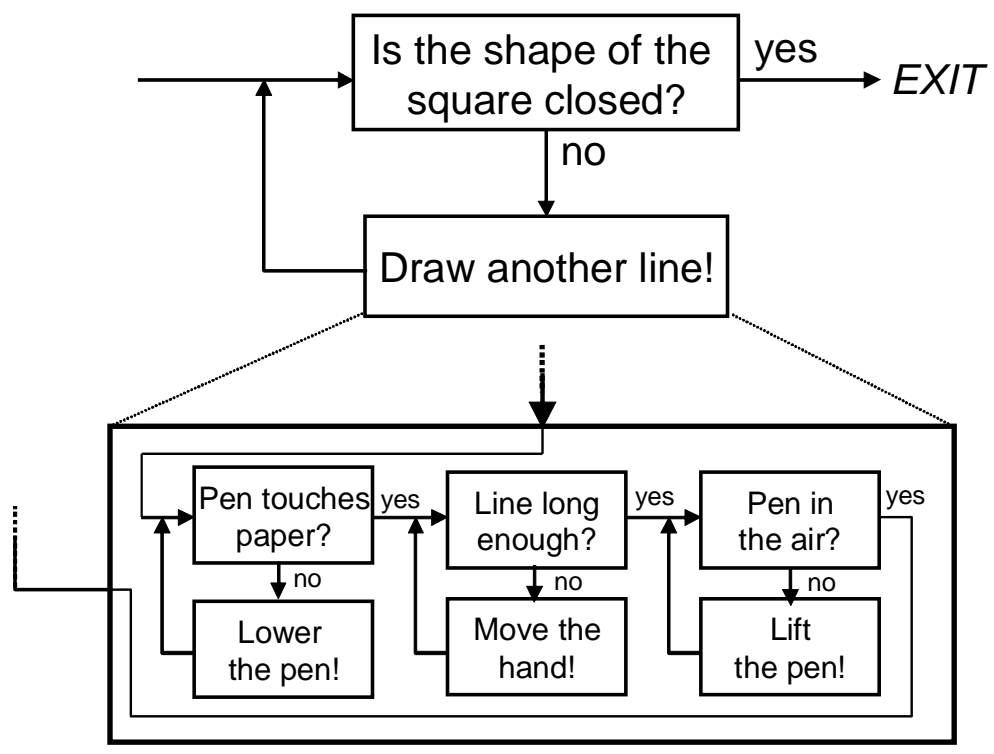

Figure 2-5: TOTE unit (according to MILLER et al. 1960)

A TOTE unit consists of only two basic steps: an operation step and a testing step. These steps can be used to describe any intentional human action. In a TOTE unit, it is firstly tested if a desired goal state is achieved and if not, then a certain operation is performed. Subsequently, another testing step is carried out to see if the operation has brought about the goal state. If this is the case, the unit is exited, otherwise another operation and testing cycle 
follows. TOTE units can be stringed one after another, forming chains, and may be embedded within one another, forming subroutines (see bottom of Figure 2-5). HACKER (1998, pp. 213) introduces $\mathrm{VVR}^{1}$ units that are control cycles similar to TOTE units ${ }^{2}$. Still, he supplements the work task as the context, in which action is embedded and which accordingly gives the conditions for action. With the help of TOTE units, even such complex processes as design may be described in a rather simple way on various levels of resolution.

How could the TOTE unit of a typical action in design look like? Let us clarify this with the help of the sketching process as a representative example. On a very basic contextual level (and accordingly, a level of high resolution), certain phases of a design process may be described as "drawing lots of single lines on pieces of paper". For each line, the operation step equals the depiction motion of the pencil with certain direction, length and position on the paper. Through the testing step, it is checked if the line constitutes the goal state of this action, e. g. if the line is straight or if the designer has been shaking during sketching. In design processes, a drawn line does not only need to fulfill demands on such a motor activity level. It also must suffice demands within the higher context of depicting certain patterns. In this case, the testing step may clarify, if the line contributes to the overall shape that the designer wants to draw. Within an even higher context, it may be tested whether the line helps to represent a satisfactory technical solution or if it introduces irrationality to the solution.

The depiction of a single line is an observable, external action and the regulation of such an action may be described by the respective "line-drawing" TOTE unit. Still, it may be (together with other actions) embedded within another TOTE unit, which regulates a more comprehensive process, such as depicting a complex pattern. This higher-level process specifies the goals of all enclosed TOTE units and the order of their execution. Likewise, this unit may again be embedded within another one and so forth. Thereby a hierarchical structure arises that contains observable actions on the bottom level, which are regulated by interwoven TOTE units of different complexity.

HACKER (1998, pp. 203) specifies the resulting structure and defines a basic level of observable actions within such a structure. The actions on the basic level are part of psychic processes of regulation on higher levels. Thereby, a hierarchical structure is formed that represents the abstract-logical structure of complex tasks. Figure 2-6 a) exemplifies this structure for the task "draw a picture of a bicycle". The structure shows in which way the complex picture of the bicycle is decomposed in single lines. Within this structure, the actual psychic processes "move from knot to knot", as shown by the thin arrows in Figure 2-6 b). The sequence of observable actions is shown by the thick grey arrows. With this consideration, Hacker lays the consideration of TOTE- or VVR-hierarchies on a solid foundation (SCHAUB 1993, p. 27) and allows for approaching the psychic processes behind action.

\footnotetext{
${ }^{1}$ VVR means "Vorwegnahme-Veränderung-Rückkopplung”, engl.: “anticipation-alteration-feedback”

${ }^{2}$ To simplify matters, we will use TOTE-units for further consideration of design action. Still, TOTE units could be replaced by VVR units in the following.
} 
a)

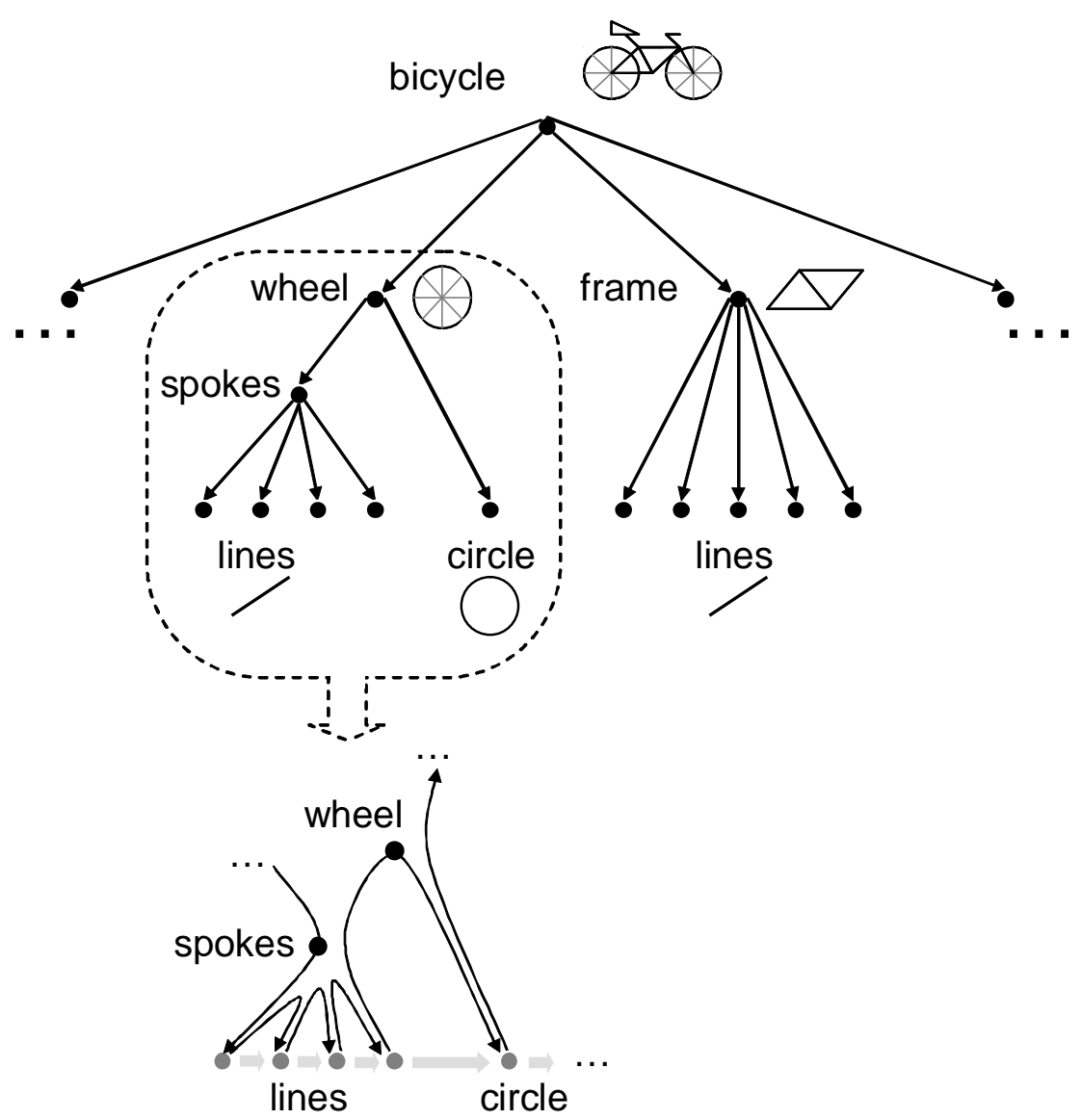

Figure 2-6: Abstract-logical structure representing the action „draw a picture of a bicycle” (a) and structure that shows the actual sequence of action (b) (according to HACKER 1998, pp. 203)

What benefit does the consideration of design via action theory provide for understanding design? This point of view allows the observation of design as a series of actual actions. Action theory is a consistent system appropriate for summarizing these actions into comprehensive processes and vice versa in an almost arbitrary way. The resulting structure of interwoven TOTE units represents the complex plans and strategies pursued by the designer. Thereby this structure is a gateway to the analysis and comprehension of the psychic processes underlying action in design.

Moreover, Hacker provides a useful approach to differentiate and categorize actions. Most people may agree that depicting a complex technical system, considered as one sole action, demand different (and probably higher) skills than drawing a (single) straight line. Different actions require different psychic regulation processes and according to HACKER (1998, pp. 237), three levels of regulation can be distinguished. The sensory-motor level of regulation holds automated actions that cannot be carried out consciously. An example for such an action is maintaining balance while riding a bicycle. Actions, which can, but do not 
need to be carried out consciously, are located on the perceptive-conceptual level. An example for such an action is throwing a basketball into the rim. The motion that needs to be performed can be described in textbooks and may be carried out very consciously during basketball practice. Still, good players will be able to perform this motion unconsciously during a game and instead concentrate on the run of the overall game. Lastly, there is the intellectual level, which contains complex actions and processes that can only be carried out consciously. Most of the actions performed in order to design a complex technical system may be regarded as actions on the intellectual level.

\subsubsection{Design as problem solving}

We have seen that design may be seen as a series of actions, which are related to complex processes of thought. We have also seen that design tasks show characteristics of problems. Cognitive psychology deals with problem solving and provides numerous approaches to analyze design.

According to DÖRNER (1987, pp. 10), a problem is defined by an undesired current state, a desired goal state (that differs from the current one) and certain barriers that prevent an instant transformation of the current state into the goal state. The existence of such barriers differentiates a problem from a task.

Notice: The colloquial use of the term "task" differs from this definition. The formulation of a problem is often referred to as a task - this especially holds for "design tasks", which usually represent challenging problems. In the following considerations, we will use both terms - "design problem" and "design task", while the first will refer to the problem characteristic of design. The latter will only refer to the formulation of a design problem.

\begin{tabular}{|c|c|c|c|}
\hline & & \multicolumn{2}{|c|}{ clarity of the goal criteria } \\
\hline & & high & low \\
\hline \multirow{2}{*}{$\begin{array}{r}\text { familiarity } \\
\text { with the } \\
\text { means }\end{array}$} & high & $\begin{array}{l}\text { interpolation- } \\
\text { barrier }\end{array}$ & $\begin{array}{l}\text { dialectic } \\
\text { barrier }\end{array}$ \\
\hline & low & $\begin{array}{l}\text { synthesis- } \\
\text { barrier }\end{array}$ & $\begin{array}{l}\text { dialectic barrier \& } \\
\text { synthesis-barrier }\end{array}$ \\
\hline
\end{tabular}

Figure 2-7: Problem barriers in dependence on the characteristics of a problem (DÖRNER 1987, p. 14)

Dörner states that problem barriers are characterized by two factors, which are "clarity of the goal criteria" and "familiarity with the means" (see Figure 2-7). Foremost, the definition of these factors shows that the character of any problem must not be seen independently from 
the problem solving person. Still, the individual perception of those factors determines the problem at hand and therefore, it determines the way a person individually deals with the problem. Let us now try to briefly clarify these factors and thereby classify common design problems according to these factors.

A classic example of a problem with a high clarity of goal criteria is a jigsaw puzzle of a photo, which is printed on the top side of the box. The clarity of a goal criterion is high if it implies a clear rule to determine whether a state is the goal or not. Furthermore, the quantity of possible states must be reduced by the application of this rule in such a way that it is possible to aim at a goal state from the beginning. Playing chess may serve as a counterexample: there are very distinct rules to identify a checkmate situation; still the number of such situations is incredibly high. In the beginning of the game, no chess player knows the checkmate situation that he might be able to accomplish. Design problems fall in the same category, as they are even ill-defined problems in many cases ${ }^{1}$. This means that in the beginning, there is no consistent system of such rules at all. In design, there may be several requirements on a future product that contradict each other. In the beginning, it is not clear in which way these requirements will be adapted in order to enable the realization of a consistent product.

The second criterion, familiarity with the means, may again be characterized by the jigsaw example. In that case, the jigsaw pieces that are spread over the table are all applicable means. The set of means is self-contained and can be overviewed - it is not allowed to saw new pieces (in fact, this would be a rather creative solution approach...). The set of means for handling design problems is practically unlimited. New technologies are developed due to efforts in research and numerous suppliers provide machine elements in countless versions. Above all, geometry provides endless opportunities to design an infinite number of technical elements with different properties. So in design, the set of means is usually not self-contained and cannot be overviewed.

We have seen that design problems may require the clarification of the goal state and may include the synthesis of elements, which are new to the designer. Nevertheless, solving such problems may also include (as sub-problems) phases, when a limited number of elements need to be combined in the right way in order to achieve a well-defined (sub-)goal state. Regarding design as a process of problem solving provides us with a number of models to analyze the procedure of problem solving. Probably, none of these models represents "the one and only" problem solving process - although one of them is even called "General Problem Solver" - and none of them fully covers design either. However, there are certain subprocesses that turn up in different problem solving models as well.

From the problem solving models proposed in psychology, we will choose two representative ones for further use: the General Problem Solver (GPS) by NEwELL et al. (1960), which was already mentioned before, and the "ARASKAM"-procedure by DOERNER (1999A). The GPS can be seen as a very fundamental approach to describe problem solving processes in the form

\footnotetext{
${ }^{1}$ According to DöRnER (1979, p. 13), the term "ill-defined problems" goes back to MCCARTHY (1956). The set of ill-defined problems is a subset of problems with low clarity of goal criteria.
} 
of algorithms. Its application on specific problems can be simulated via computer programs. This model is based on observations and describes the way operators are chosen and executed in order to achieve a certain goal state. It consists of three processes that are embedded within one another (see Figure 2-8).

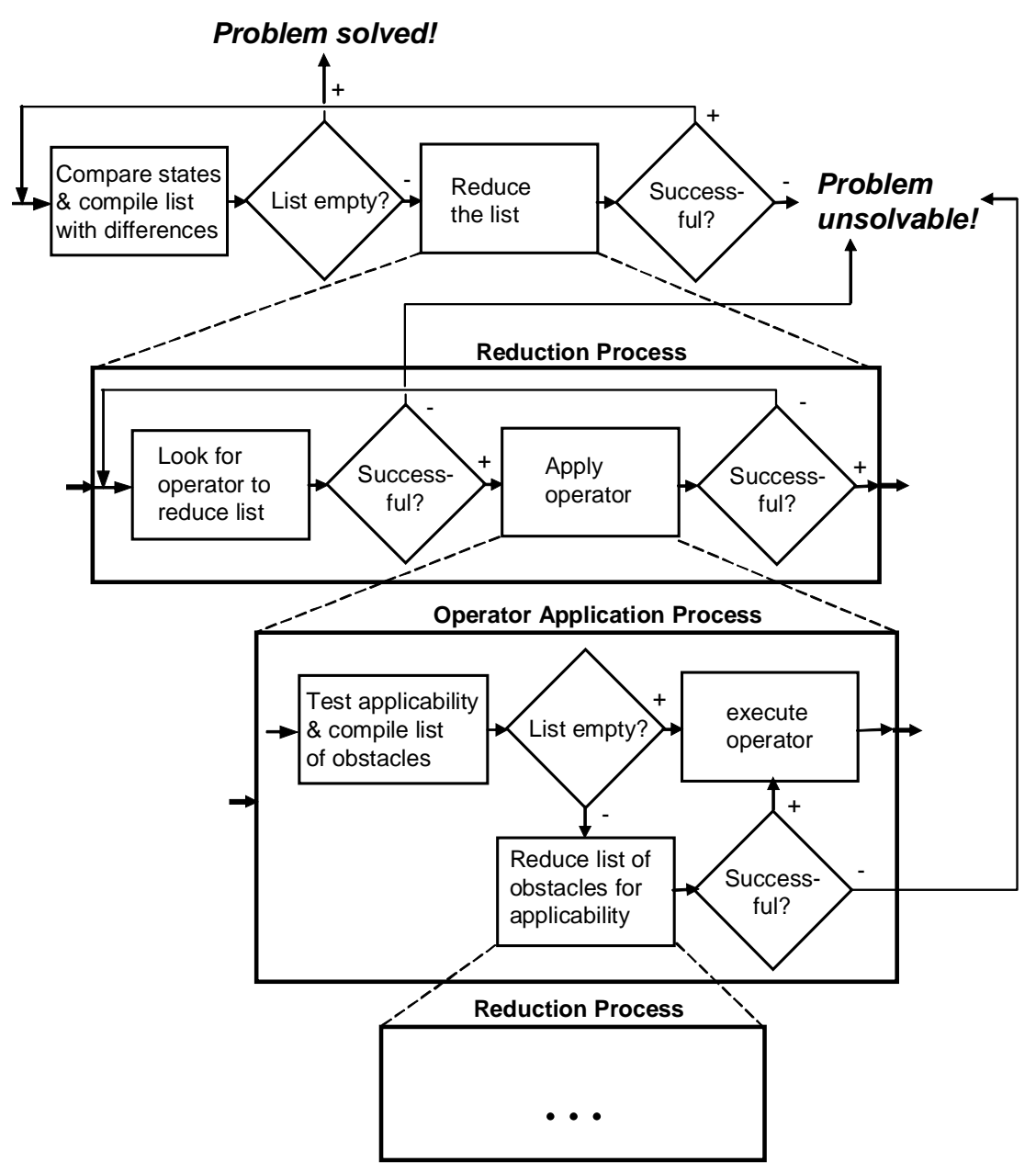

Figure 2-8: General problem solver (NEWELL et al. 1960)

The transformation-process compares the actual state to the goal state and compiles a list with the differences. If there is no difference on the list, then the problem is solved. Otherwise the reduction-process is accessed, which looks for an operator that is capable of reducing the list of differences. If no such operator can be found, then the problem is considered to be unsolvable. Otherwise the operator-application-process is accessed, which tests the applicability of the operator in the actual situation. If the operator fits the situation, it is executed. If the situation does not allow the application of the operator, then a subordinate reduction-process is accessed that in turn tries to alter the situation. As soon as the operator becomes applicable, it is executed. Thereby a new actual state is produced, which in turn 
serves as input for the transformation-process on the upper-level. So the process starts over again until all differences between actual state and goal state are eliminated.

The GPS is (unfortunately) not as general as its name implies. Right at the beginning of each cycle, the properties of the actual state and the goal state are compared ("means-endanalysis"). Of course, this requires knowledge about the goal state's properties, which shows that GPS is only applicable to problems with high clarity of goal criteria. Furthermore, it is dependent on a self-contained database of operators. There is no process for the creation of new operators; hence the GPS applies to problems with a high familiarity with the means (DÖRNER 1987, p. 76).

Although we have seen that the GPS does not apply to design problems comprehensively, there are some basic characteristics that do apply to design problem solving. Parts of GPS are recursive, which means that a sub-process may contain itself on a lower level of resolution. In case of the GPS, the operator-application-process contains a reduction-process, which contains another operator-application-process in turn and so on. We will see later on that recursivity is a characteristic of many problem solving strategies, even those that are especially intended for design. Recursivity implies generality to a procedure, since it becomes applicable on different levels of resolution. Another basic characteristic of the GPS applies to many problem solving processes as well. The GPS is processed in loops respectively cycles so the sequence may be repeated as often as necessary. Such an iterative character implies certain flexibility to react upon failure without adding more complexity to the procedure in principle. Lastly, we have seen that a situation may be altered in the course of GPS processing just to enable the application of an operator. This means that a sub-goal is established and followed up interim. The creation of sub-goals helps to reduce the complexity of the process.

Other conceivable problem solving strategies are not implemented in the GPS, yet their benefit for problem solving seems to be obvious. DÖRNER (1987, pp.66) lists several useful strategies for problem solving: Instead of establishing a sub-ordinate goal to make an operator applicable, it may be useful to look for other operators first. An algorithm could be integrated that decides which one of those possibilities is more favorable under actual circumstances. Even when it is possible to reach a certain (sub-)goal, it may be useful to change the goal. During the course of problem solving, it may show up that a different sub-goal opens up a better way to the same overall goal. Likewise, it may be useful to change the starting-point of the actual (sub-)process, e. g. by going back one cycle of transformation and then head into a different direction. This may be a useful measure to avoid getting stuck in a "one-way-street".

A problem solving process may be planned at first before it is carried out de facto. If that is the case, then going from actual state to goal state is not the only direction to work on a problem. It is also possible to start from the goal state and try to reach the actual state by "reverse-execution" of operators. Even a mixed direction of search is imaginable and may be advantageous. Starting in an alternating way from both actual state and goal state in order to make both paths meet in between may be called "two-sided problem solving" (LEHMANN 1972, p. 51) and helps to reduce the search space as shown in Figure 2-9. 


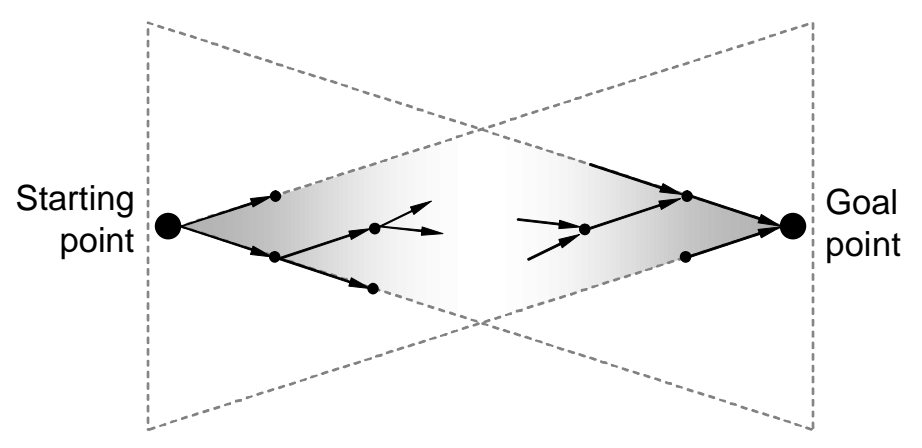

Figure 2-9: Reduction of the search space with the help of two sided problem solving (LEHMANN 1972, p. 51)

Although The GPS and its variations do not apply directly to design problems, we have seen certain characteristics that will recur in procedures intended for design. We will now have a look at the so-called "ARASKAM" procedure by DÖRNER (1999A). It can generally be used for problems of composition, and DÖRNER (1999B) exemplarily applied it to design. "ARASKAM" is a German acronym for "general, recursive, analytical-synthetical conceptamplification". It may be described as a dialogue, starting with a question, that the designer asks himself. Revealingly, the chapter of Dörner's book, in which this ARASKAM is described, is called "The inner conversation of the soul with itself" (DÖRNER 1999A). Solving problems in dialogue form seems to be a characteristic of creative problem solving processes since it is also described by others in this context. SCHOEN (1983) describes professionals conducting dialogues with given situations as a source of creativity, WULF (2002) describes design as a "discursive process."

DÖRNER (1999B) exemplifies a design processes carried out by means of the ARASKAM procedure (see Figure 2-10). The design task is (briefly formulated): "Design a mounting in order to attach an optical device to the wall!" Now the designer may ask himself "What actually is a mounting anyway?" The answer to that question may be a subordinate concept, such as "A flange with a wall plug and a screw is a specific kind of mounting." It may also be a superordinate concept such as "A mounting is some kind of fixture or connection." In that case, the superordinate concept can be specified again, such as "magnetic force can provide a fixture as well." Thereby, a coadjunction ${ }^{1}$ of the concept "mounting" is being discovered.

\footnotetext{
${ }^{1}$ A coadjunction is a "neighboring" concept, which falls in the same subordinate concept, but is different from the initial concept. "German sheperd dog" is a coadjunction to "bull terrier", since they are not the same, although they are both dogs.
} 
The initial question offers several ways to gain specific elements for a design solution - either directly or indirectly - via sub-processes of abstraction and concretion ${ }^{1}$. According to Dörner, these processes are mainly carried out by means of imaginary terms respectively words. Still, there are also pictorial imaginations involved in the overall process. The elements, which have been produced as terms, are converted into pictures and composed to an overall picture of the solution approach and its surroundings. This picture may emerge on the "inner" (visuospatial) sketchpad (see page 12) or may be sketched on a "real" piece of paper. By means of this picture, the composition is tested for its compatibility with the requirements on the design solution. If this test fails in any aspect, sub-cycles of the ARASKAM-procedure search for new elements in order to substitute faulty parts of the solution - in the worst case, the whole solution is revised.

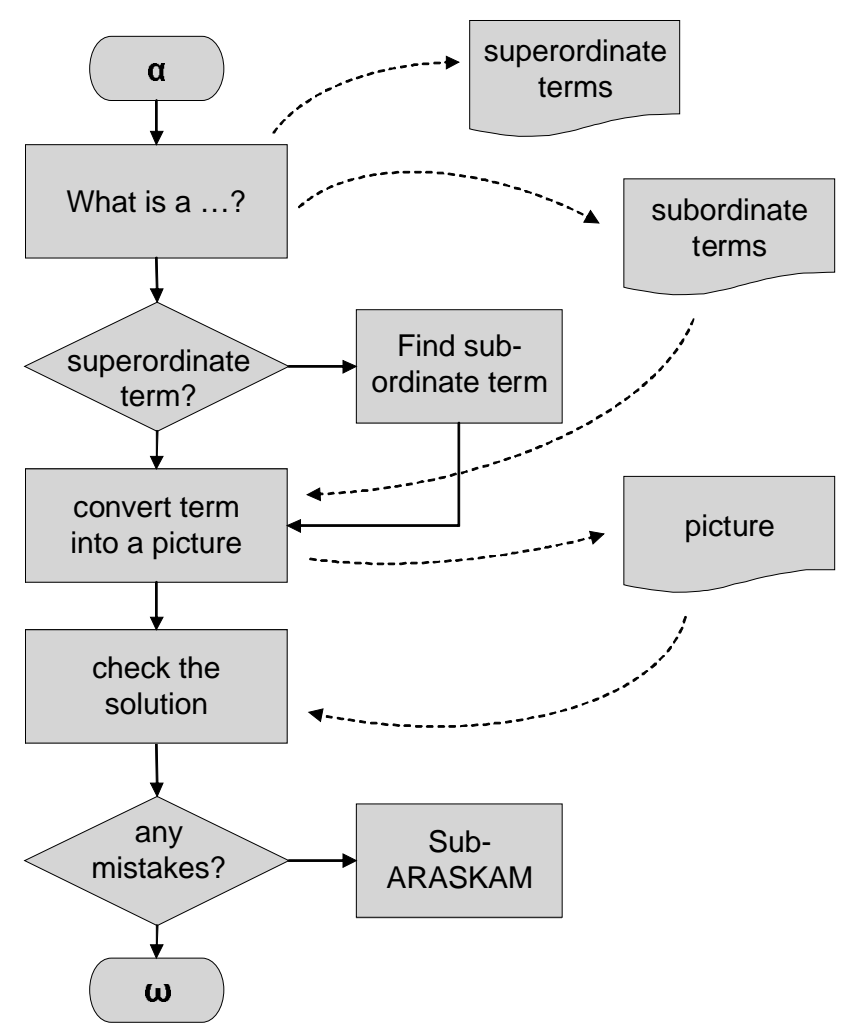

Figure 2-10: ARASKAM-procedure, exemplified by the design of a mounting for an optical device (DÖRNER 1999B)

An ARASKAM procedure may contain itself again on a lower level and is recursive therefore (as a reminder - that is where the letter R in ARASKAM comes from!). Recursivity has

\footnotetext{
1 Dörner names those processes of abstraction and concretion in this context finding "superordinate" and "subordinate" concepts.
} 
occurred within the GPS as well, so this seems to be a rather general characteristic of models for problem solving processes.

We will see later on, that some characteristics of problem solving processes in general also appear in procedure plans for design in particular. Since it is generally agreed that design is (at least partially) problem solving, this may be seen as an indicator for the usability of a design procedure plan. But beforehand, we will view design from yet another perspective, which is creativity. After all, design processes should be carried out in a way that the occurrence of new ideas is likely to happen or at least not be suppressed.

\subsubsection{Design as a Creative Process}

The term creativity is only vaguely specified, but - probably for this reason - often used (and abused) in the business area. Psychologists mainly agree on two criteria to distinguish creative processes from others: Solutions emerging from creative processes shall be new and/or unusual and they shall be useful (HUSSY 1984B, p. 65). DöRNER (1987, p. 79) states that creative problem solving represents a special case of solving problems with low clarity of goals. So in the strict sense, the viewpoint on the design process that is addressed by this headline has been covered by the previous chapter already. Still, the term creativity raises expectations about a very important aspect of conceptual design: the idea. This "phenomenon", as well as the conditions, under which it may occur have not been addressed properly, yet. We will do this in the following paragraphs against the background of creativity.

According to EHRLENSPIEL (2003, p. 382), creativity is "the ability of a person to create ideas, concepts, products and combinations which are new in essential aspects and have been unknown to the person so far". This definition may be supplemented by definitions from psychology, which often point out that the result of a creative process should comply with social requirements by being "useful for society" (DORSCH 1982). In engineering design, this term can be replaced by "meeting the requirements to the future product".

From a purely rational angle, it is hard to imagine that one may depict something that he has not known before ${ }^{1}$. The mechanisms that were described in the previous chapter are capable of finding elements for potential solution by following up associations between terms (such as abstraction and concretion). Still, these elements are known and they are somewhat associated to the problem, otherwise these mechanisms would not work.

Therefore, in Ehrlenspiel's definition, the term "combinations" seems to be essential. A new product should provide new functions, which are realized by a new combination of machine elements. Even if these elements are known, the number of possibilities for combination is virtually infinite. The basic principle from Gestalt psychology can be applied to technical

\footnotetext{
${ }^{1}$ In natural science, there are laws of conservation, which claim that the outcome from a system cannot be bigger than what has been included in respectively fed to the system before!
} 
solution: The whole is more than the sum of its parts (KOFFKA 1935, p. 176) ${ }^{1}$. Accordingly, the novelty of a solution is not determined by the novelty of its elements, but by its overall gestalt. For this reason, the mechanisms described in the previous chapter may still serve for developing creative solutions.

However, there is an important mechanism of solution development that may lead to elements of a solution that have not been associated to the problem before and this is conclusion by analogy (DREISTADT 1968). Starting from one known concept, another concept may come to the designer's mind that is somehow similar in some outward attribute. From that outward similarity, the hypothesis is stated, that there are similarities in behavior as well. And sometimes this hypothesis proves (at least partly) true and a solution element can be transferred from a completely different area of expertise. The nowadays "popular" method of bionics is an example for provoking such a conclusion by analogy. While working on the problem to optimize the air resistance of a plane, the designer might be reminded of a shark because of the similarity in shape or maybe because the gliding motions resemble. The shark has a unique skin surface that provides this animal with a low flow resistance. The hypotheses of analogy then would be: "a plane with such a surface might also have a low air resistance!" Ideas from other disciplines can seldom be applied directly - we will probably never make a plane grow skin, less than ever with a defined surface ...but a lacquering is somewhat similar to skin (here we got the next analogy!). If we derive the principle behind the low resistance of shark skin, we may be able to apply it to the lacquering.

The advance from this process is that it is able to link into a domain that is completely different from the one that contains the problem. Any similar feature between two concepts may be sufficient to awake an analogy and it is not necessary that there has been any association before - the association is being established by the analogy. This process may be carried out systematically by using the method of bionics or synectics (GORDON 1961), but it may also be initiated by accident. The most popular story of accidental analogy probably is the discovery of the ring-shaped structure of benzene by Kékulé. It is said that he had the vision of the molecule's configuration while sitting by the fireside and watching sparks flying in curved trajectories (see for example DöRNER 1987).

If we go back to creativity and to the analogies that are awoke by this term itself, another accidental factor in problem solving comes to one's mind: the sudden idea. Everyone probably knows from experience that ideas can unexpectedly occur in peculiar places and days after the initial contact with the problem. This makes it so hard to investigate this cognitive mechanism. And this probably is the reason why DöRNER $(1987$, p. 80) once called the sudden idea one of the "darkest spots in cognitive psychology".

The sudden idea does not emerge purely accidentally. OSBORN (1963) portrays cases of idea generation that involved several stages of development. WALLAS (1926) suggests four different phases of idea generation: Usually in the beginning, there is an unsuccessful

\footnotetext{
${ }^{1}$ The complete quote from KoFFKA (1935) is: "It has been said: The whole is more than the sum of its parts. It is more correct to say that the whole is something else than the sum of its parts, because summing up is a meaningless procedure, whereas the whole-part relationship is meaningful."
} 
preoccupation with the problem - in view of the idea to come, this phase is called preparation. After this engagement, the problem is laid aside - actually as well as mentally and the incubation period follows. This period is what makes an idea "sudden", since usually there is no perceptible further elaboration of the problem during this period. Often there is also no noticeable reference or clue to the problem within the person's surrounding. Then the idea emerges seemingly out of nowhere - significantly, this moment is called illumination. So the process of "having a sudden idea" is organized in 3 stages so far:

1. Preparation - unsuccessful preoccupation with the problem

2. Incubation - no preoccupation with the problem

3. Illumination - sudden and unforeseen idea

The characterization of the stages by the means of such terms as "unsuccessful" and "unforeseen" shows why this process is so hard to control. As a logical consequence, the last stage has been added:

4. Verification - testing the idea on its usability

This is the only stage that can be carried out systematically. Although it is difficult to analyze the other stages scientifically, there are assumptions according to the mechanisms leading to a sudden idea.

DÖRNER (1987, pp. 92) interprets assumptions from POINCARÉ (1973) in a way that the right solution - the idea - has already been developed during the preparation phase (among other wrong solutions). Working on a problem can be seen as the attempt to form a picture that contains the right elements with the right linkage in between. During unsuccessful problem solving in the preparation phase, many new linkages have temporarily been established. In turn, many of those linkages have been regarded as being inapplicable, yet they have not vanished. So at the end of this process, an unclear picture has emerged, which shows a lot of elements and links. The picture may contain the correct solution, but it does not obtrude, since it is absorbed by the "background noise" of other elements and links.

During the incubation period, links and elements in the mental picture are forgotten bit by bit. Dörner assumes that those links, which form the correct solution, are less affected by the process of forgetting than others. How should this kind of "intelligent oblivion" work? The more often a link is activated, the stronger the connection is and the more resistant it is against vanishing. Dörner supposes that the elements of the correct solution are each activated more often than others during the preparation period. The reason therefore may be that every one of those elements has been identified as being necessary for a correct solution itself. Yet, they just have not been activated together at once, otherwise the correct solution would have been recognized. Since each activation of any element also activates its links to other elements, the connections between the correct elements are activated more often (alternately from both sides). Therefore, after a certain process of oblivion, the links between the correct elements "come forward" - all at once for the first time. The recognition of this picture is the moment of the sudden idea. 


\subsection{Ways to Support the Design Process}

Designers do not work self-determined, independently and on their own. They are integrated in a working environment, so they work in teams and those teams are part of superordinated organization, such as the department of a company. The individual contributes partial work for the team and the team contributes partial work for the organization (HACKER 1998, p. 49). The designer (as well as the team) is supported in his work by design methodology. Integrated product development (EHRLENSPIEL 2003) provides different forms of support, along with the appropriate framework of product development that allows for their specific application. Support from integrated product development relates to (LINDEMANN \& KLEEDÖRFER 1997, p. 115):

- cost management

- project management

- process organization

- strategies and methods

- procedure plans

- design media and tools

We will address the latter two in the following paragraphs and we will regard procedure plans first. Procedure plans recommend a certain structure of work steps and give partial goals for those steps (GIAPOULIS et al. 1995, p. 477). In the previous chapter, we became acquainted with certain procedures for problem solving. They were supposed to describe actual problem solving behavior, but they may also serve as prescriptive procedure plans for problem solvers to improve their performance. Design methodology provides procedure plans that are intended for the increase of design performance in particular. We will exemplify some characteristic ones now.

The design procedure according to VDI-GUIDELINE 2221 covers the whole design process from start to end and divides the process into phases. These phases differ in the degree of abstraction of the product documentation in progress. The degrees of abstraction and the corresponding phases are shown in Figure 2-11. VDI 2221 shows the goal for each design phase and integrates the phase in the overall course of the design process. Still, it does not provide support for the specific procedure of the designer within a phase. 


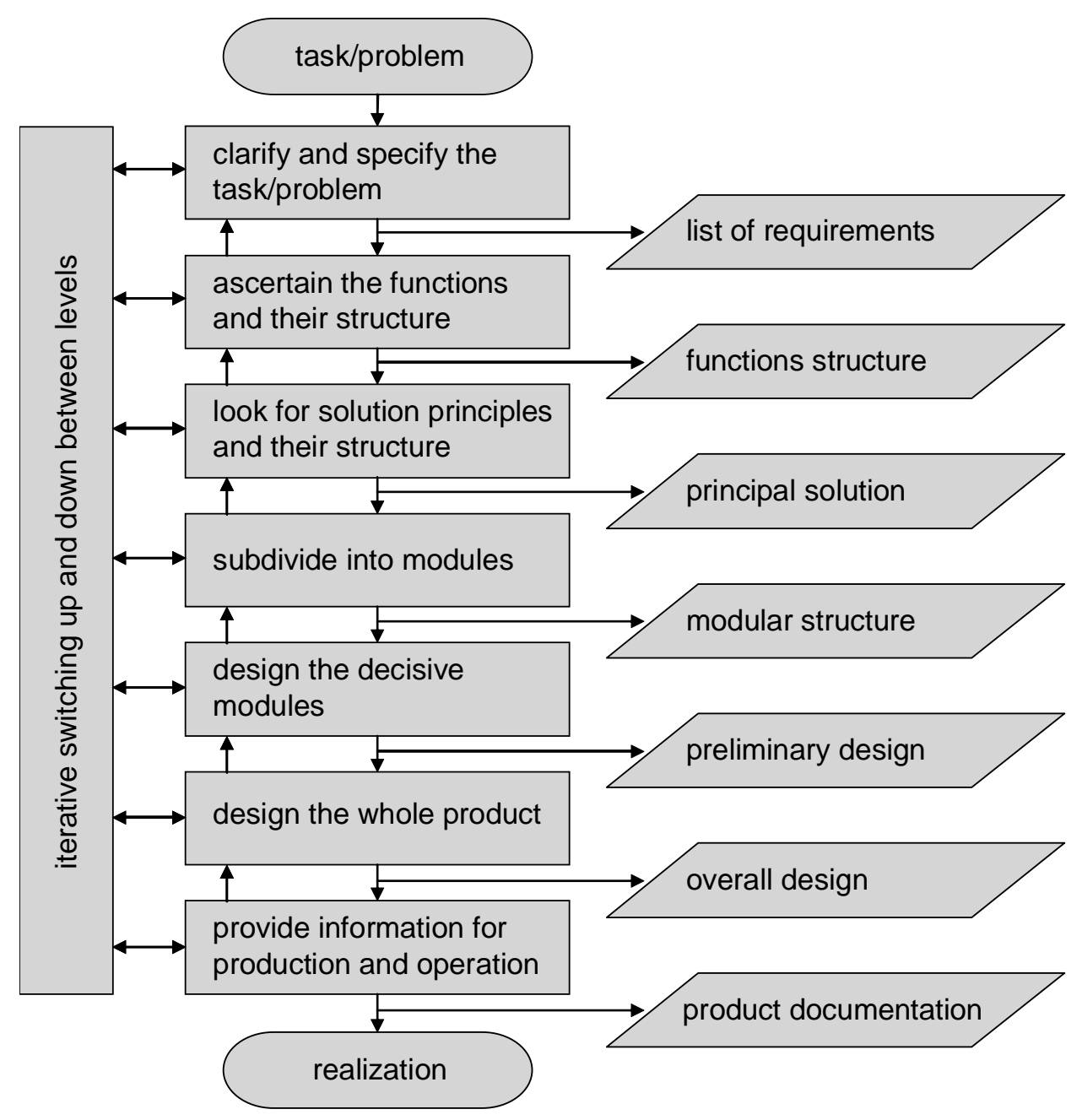

Figure 2-11: Procedure in design according to VDI 2221 (1993, p. 9)

The Munich Procedural Model MPM (LINDEMANN 2003C) aims at problem solving within a design process. In contrast to VDI 2221, it is formulated rather independent from the specific object of the overall design process. Hence, it can be used on various levels of resolution at any time within a design process. The coverage of the model's application depends on what is regarded as a problem by the designer and it may be applied to any kind of work step within the product development process. 
The MPM offers seven work steps, which are:

- plan the target

- clarify the target

- structure the target

- find solutions

- analyze properties

- select the solution

- ensure achieving the target

The MPM is a very flexible suggestion of work steps that may be processed in a flexible order in accordance to the actual situation, although there are some preferable sequences. Figure 2-12 shows this with the help of dots, each representing a certain work step. The "dots" can be processed in any desired order to reach the goal state.

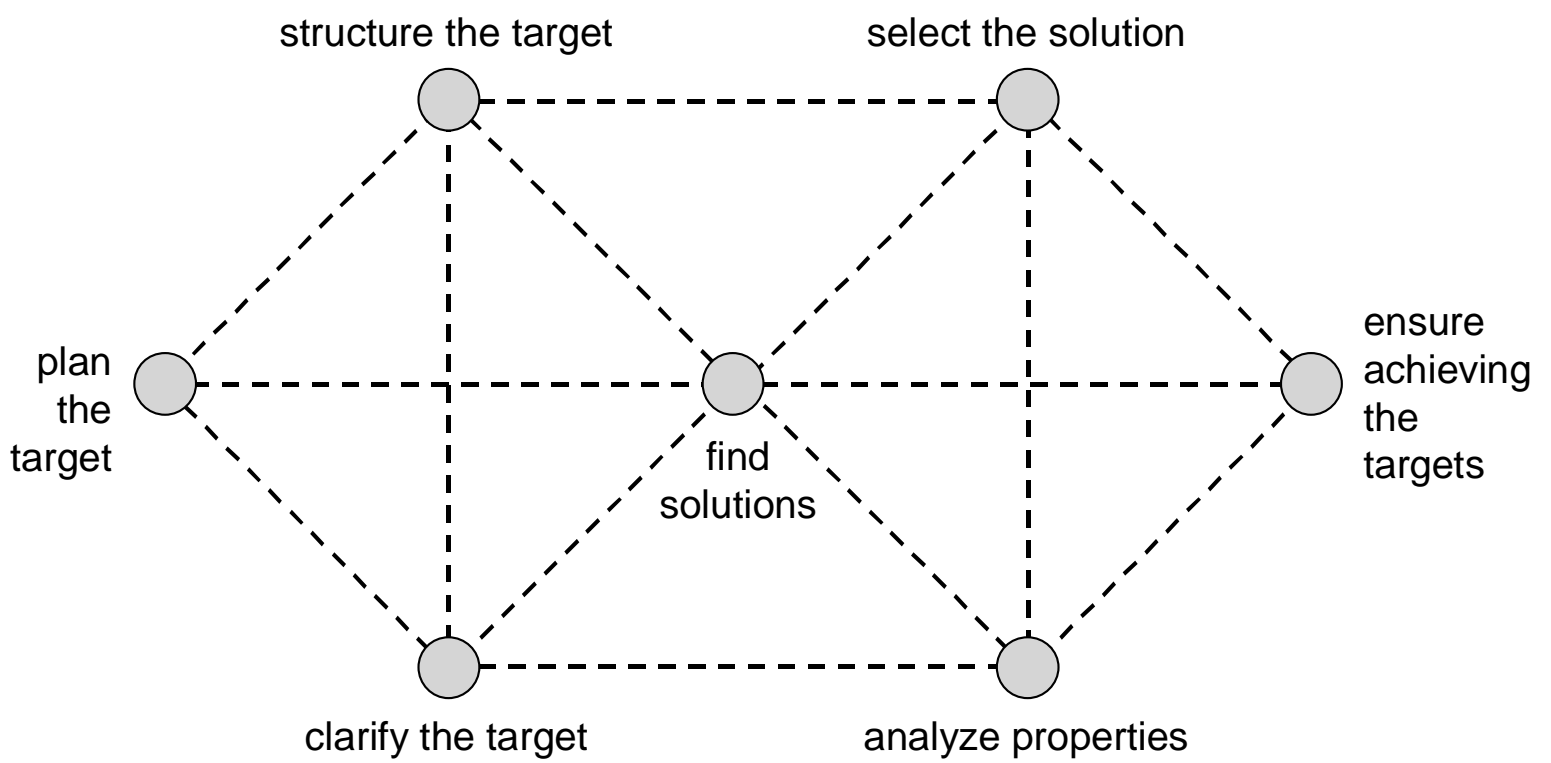

Figure 2-12: Munich Procedural Model (MPM) according to LINDEMANN (2004)

In contrast to VDI 2221, MPM is recursive. The MPM can itself be applied for processing its own work steps, in case that a sub-problem occurs. The MPM is based on the procedure-cycle for problem solving by EHRLENSPIEL (2003, pp. 79), which consists of the three main steps clarify the problem, search solutions and choose a solution (see Figure 2-13). 


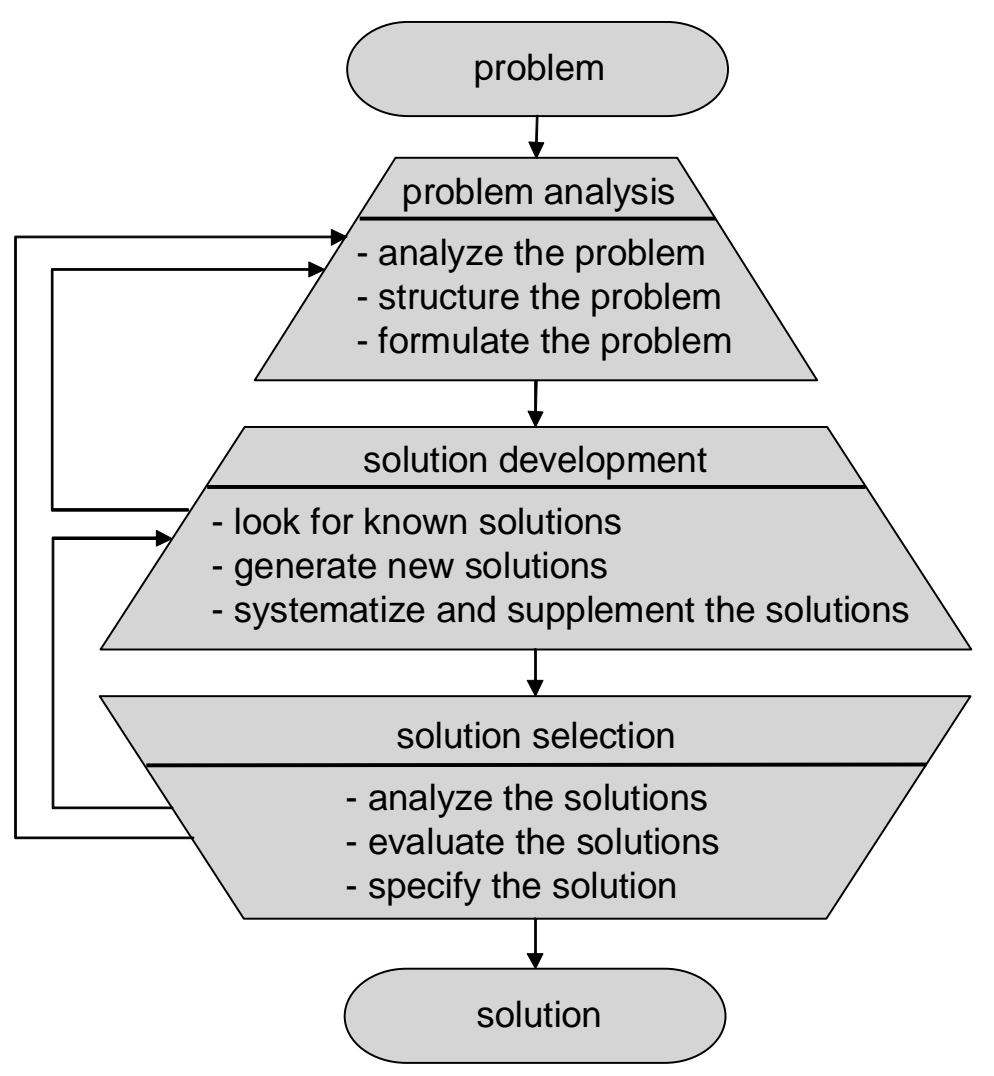

Figure 2-13: Procedure cycle according to EHRLENSPIEL (2003, p. 83)

Both these procedures for problem solving in design mirror some basic operations of problem solving in general: synthesis, analysis and comparison. Furthermore, the objects of these operations are aspects of the actual state and the goal state. Operations can be processed in an alternating matter, which results in algorithms similar to the ones described in a previous subchapter (see 2.2.3). So we see that essential characteristics of problem solving procedures from psychology appear in design procedure plans as well. This results from the fact that to some extent, design is problem solving indeed. However, such design procedure plans advise the designer what to do at a certain time, but they do not tell him how to do it.

As we have seen before, the crucial "working memory" of the human mind is severely limited. From a theoretical point of view, the use of external design media may be considered as being nearly indispensable when complex products are designed. Design media, which represent the current state of a product definition, determine in which way this definition can be manipulated. Therefore, design media crucially influence how design action is actually carried out. Design tools are meant to help the designer in carrying out such design activities more efficiently and effectively (in some cases, media and tools can even hardly be distinguished from another). 
In the next chapter, an extensive survey is presented, which investigates the actual use of different design media and tools in mechanical engineering. On the basis of this survey, we will elaborate the purposes for their use along with the benefits and drawbacks that arise from their application. These results reveal the designers' demands, needs and wishes according to their media and tools.

Both, the theoretical conclusions from the preceding considerations and the practical insights from the next chapter will then provide us with a framework that allows us to actually fathom out the mechanisms that underlie sketching. 
This book is available at Verlag Dr. Hut, München, www.dr.hut-verlag.de (ISBN 3-89963-176-5) 


\section{Media and Tools for Conceptual Design in Mechanical Engineering}

The working memory is the area of the human mind where creative reasoning takes place. Therein, information can be altered, interrelated, combined and decomposed. It is the area where new information can purposefully be created and, unfortunately, this area is rather limited. As we have seen before, the working memory can hold about seven information units, so-called chunks, at the same time. In order to handle more complex systems comprehensively, information units need to be retained temporarily within an external storage. It is clearly apparent that relief of the working memory is one basic function of external product representations in design (SACHSE \& HACKER 1997).

Sophisticated design tools such as CAD (Computer Aided Design - we will regard this in greater detail soon) provide complex product representations along with numerous possibilities for their manipulation. Accordingly, CAD is a tool that directly effects a momentary product definition, such as it allows the generation, representation and alteration of product features. In this thesis, we will concentrate on such design tools (hence, we will disregard tools for management of design processes for example). Moreover, we will confine our consideration to those tools that are applied on conceptual design sequences. Yet before we examine which digital and analogous tools apply to conceptual design in particular, we need to clarify the term tool.

The common use of "tool" in the engineering design domain does not correspond to the comprehension that we will adapt in the following considerations. Usually, design tool stands for any kind of device, auxiliary means or software that aids in accomplishing a design task. At first, we extract two very basic functionalities of such design tools: representing a product definition and facilitating the processing of such a definition. One might argue that representing a product definition should be seen as just one special way of processing it. In design, it is necessary to see representation in a much broader sense. Due to the limitation of the human memory, the product definition often would not be there at all without its representation. Certain design tools (such as CAD and sketches) serve as a medium for the representation of a product definition. Hence, they strongly influence the definition itself, since certain media can only represent information in a certain way. In the following chapters, we will concentrate on design media in the first place, yet we will still pay attention to the procession of the product definitions that are represented by these media.

As the heading of this chapter reveals, we will still keep up the use of the term "tool." A tool is regarded as any device that supports, facilitates or carries out the procession of a product definition, hence the manipulation of a medium. Many media serve as tools as well, since they provide tool-like characteristics. Both these classifications should not be seen as being mutually exclusive; they may label different characteristics of one and the same object. Actually, we will even distinguish tools into instruments and automats. The term instrument corresponds to the original sense of the term tool as any kind of device that intensifies human action. In contrast to a tool, an automat substitutes human action (HACKER 1998, pp. 159). We 
will discuss the subdivision into these functionalities later on, but the reader may keep this differentiation in mind meanwhile.

In the following chapter, we will have a detailed look at the actual use of design media and tools in industry. On this account, a survey among professional designers is presented, which investigates the benefits and drawbacks from the use of the most common design aids. Yet beforehand, we will briefly illustrate those media and tools so all readers share a common understanding on the technical backgrounds.

\subsection{Illustration of Common Media and Tools for Design}

Sketching is a major subject of this thesis - still, we will refrain from explaining or even defining the design medium "sketch" in detail at this point. Actually, one may see a sketch as being no more than a piece of paper, complemented by a pencil and sometimes there is a rubber as well - and that is it. Any further characteristics of sketches in particular accrue from the way this medium is used. We will explore the use of sketches for conceptual design in great detail later on and thereby, the reader may gain his own definition of the design medium sketch. Before we analyze the way that media and tools for design are actually used in industry, we will turn our attention to common digital ones.

Design media need to be viewed from two points of view: They may serve as contemporary representation of a provisional result as well as a "container" for the final result in order to transfer it to subsequent processes. The latter point of view is inevitable since in mechanical engineering the result of a design process needs to be manufactured. Therefore, the media needs to fulfill certain requirements resulting from manufacturing processes. These processes mainly base on digital product information. Moreover, the management of product and process data may be rationalized when digital data is available. Therefore, we will view the description of CAD, Virtual Reality and Rapid Prototyping in the following chapters. Those readers, who feel that they are fairly familiar with these design aids may skip these chapters and go on reading with chapter 3.2.

\subsubsection{CAD ${ }^{1}$}

CAD is meant to serve as a tool for supporting the designer's reasoning, but in the first place, it surely is a medium for modeling a future product. Early CAD-systems were developed as digital equivalents of drawing boards; hence they represented lines in order to model 2dimensional (sectional) views on the future product. Emanating from those 2D-CAD-systems, so called $2 \frac{1}{2} 2 \mathrm{D}$-CAD-systems were developed, which allowed to attach information about the extension in the 3rd dimension to a 2-dimensional cross section. Then, "true" 3-dimensional systems were created, which allowed for the definition of lines in space in order to model the edges of a 3-dimensional body. As a next step, surfaces in space could be defined that

\footnotetext{
1 The descriptions according to CAD in this subchapter follow LINDEMANN (2003A, part 3 and 4) unless otherwise noted.
} 
represented either thin sheet metal parts or the boundary surfaces of a voluminous body. Consequently, the next generation of CAD-systems annotated surfaces with a vector that indicated the inside of a body's boundary surface. Hence, solids could be modeled that included information about physical properties of the future product, such as its mass. Actual development aims on integration of all product features into the digital model, e. g. information about its operation and recycling. Yet, 2D and 3D systems are both in use in mechanical engineering industry (as we will regard in detail later on in chapter 3.2).

Early 2D-systems required the input of the coordinates of the starting point and the endpoint for each line (together with additional information in case of curved lines). Nowadays, the designer can choose numerous standard shapes (rectangles, circles, etc.), which can be defined with rather little effort by means of a graphical user interface.

Actual CAD-systems using 3D-solids allow for the definition of bodies by (see Figure 3-1)

a) choosing (relatively simple) given volumes, called primitives or CSG (= Constructive Solid Geometries)

b) expanding 2-dimensional cross sections into the $3^{\text {rd }}$ dimension (the cross sections are previously defined similar to shapes in 2D-CAD systems),

c) combining solids with the help of bool's operations or

d) joining surfaces together.
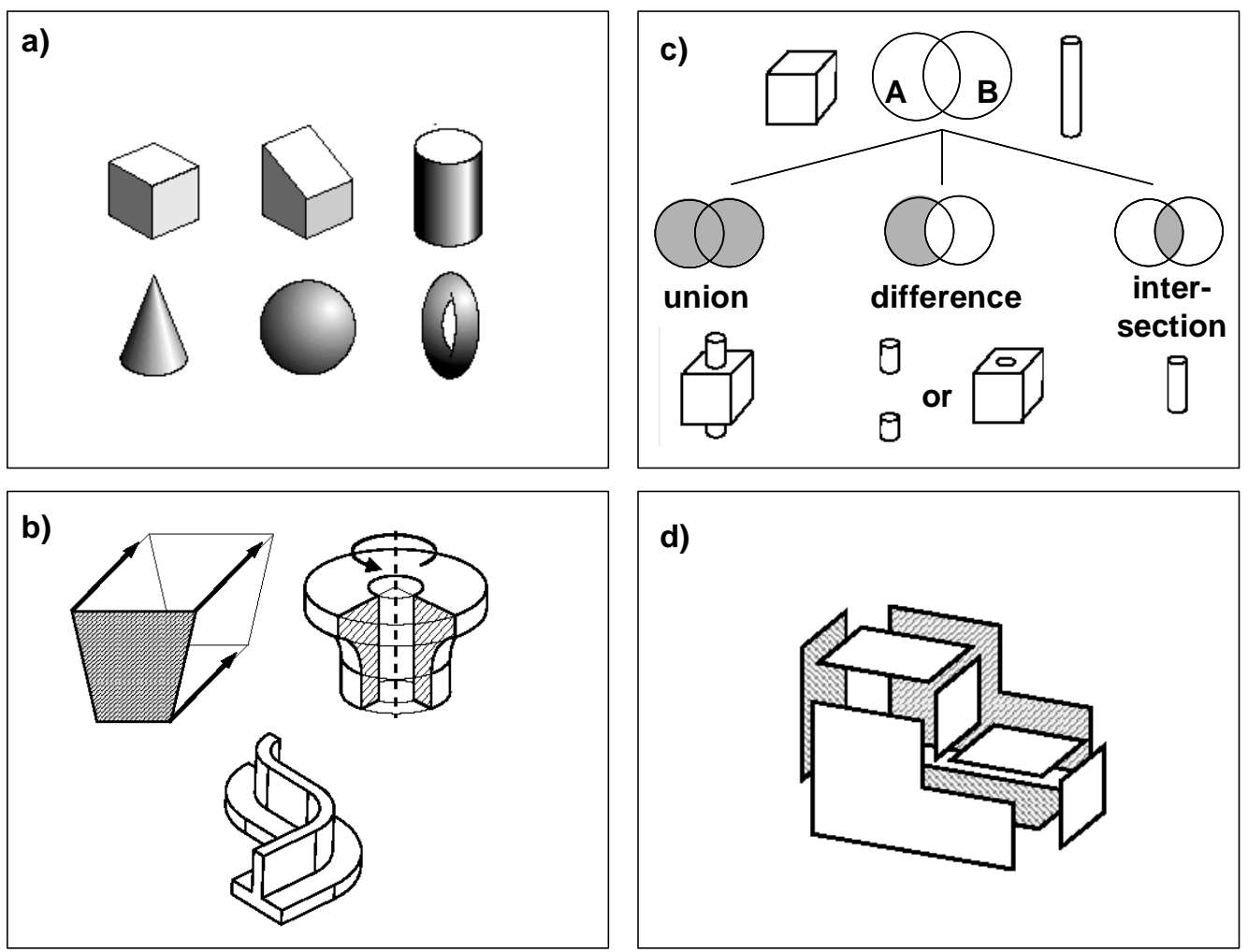

d)

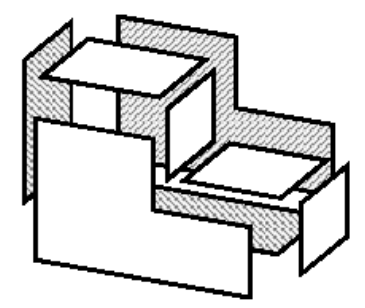

Figure 3-1: Different principles of body generation in CAD 
Moreover, most actual systems provide features in order to make modeling the product definition more efficient. In the context of CAD-modeling, a feature is an aggregation of a number of attributes that the designer can specify as a whole. Features may contain shape information and/or certain semantics. Examples for pure shape-features are primitives that are mentioned above. A cylindrical body can be created by specification of diameter, length and information about its position; it is not necessary to model the body by defining and combining all its boundary surfaces. A pure semantic feature is a draft symbol indicating the processing of a surface. This feature does not alter the geometry of the CAD-model (the surface structure of a product is not geometrically represented in CAD), but it provides information about the process of manufacturing. A feature that contains shape and semantic information is a tapped hole with a drilling cone. The use of this feature alters the model's geometry and in addition, it specifies the manufacturing process since it determines the necessary drill (see Figure 3-2).

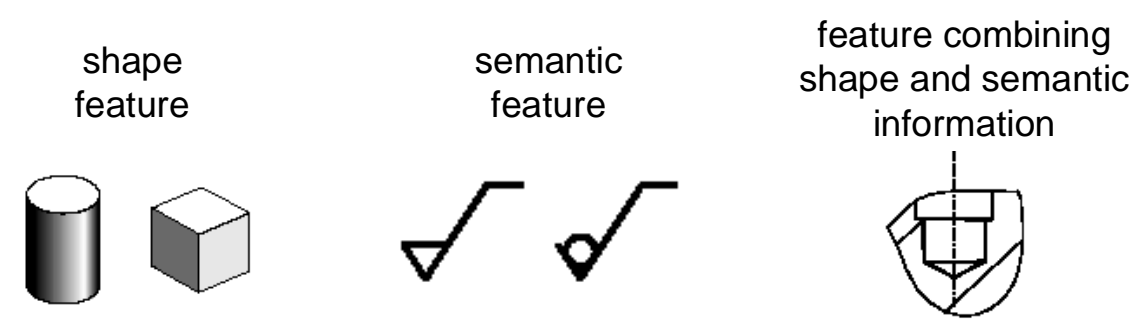

Figure 3-2: Features in CAD

In order to facilitate the designer in making quick changes to a CAD-model and in generating product variants from a given $\mathrm{CAD}$-model, parametric links may be added to CAD-models. Parameters within a model may be geometric (coordinates, measures, etc.), technological (tools, feed rate, etc.) or may be related to the material (density, stability, etc.) for instance. Such parameters may be connected to each other and thereby, a dependency of one parameter from another may be created. CAD-systems provide parametrical dependencies in order to enable the designer to model his design intent, e. g. "this edge should be parallel to that edge" or "this measure should be double that measure". Changes made to one parameter then bring about certain changes made to other parameters automatically. 
Yet, it seems that parametric design, that is somewhat obtruded by actual CAD-systems does not apply to all design activities. When innovative design is in demand, the parametric fortunes of computers cannot be used much until solutions for optimization exist (OTTOSON 2003). Figure 3-3 illustrates this matter. The "design window" shows the application of CAD in dependency from levels of completion and abstraction of the respective (part of the) product definition.
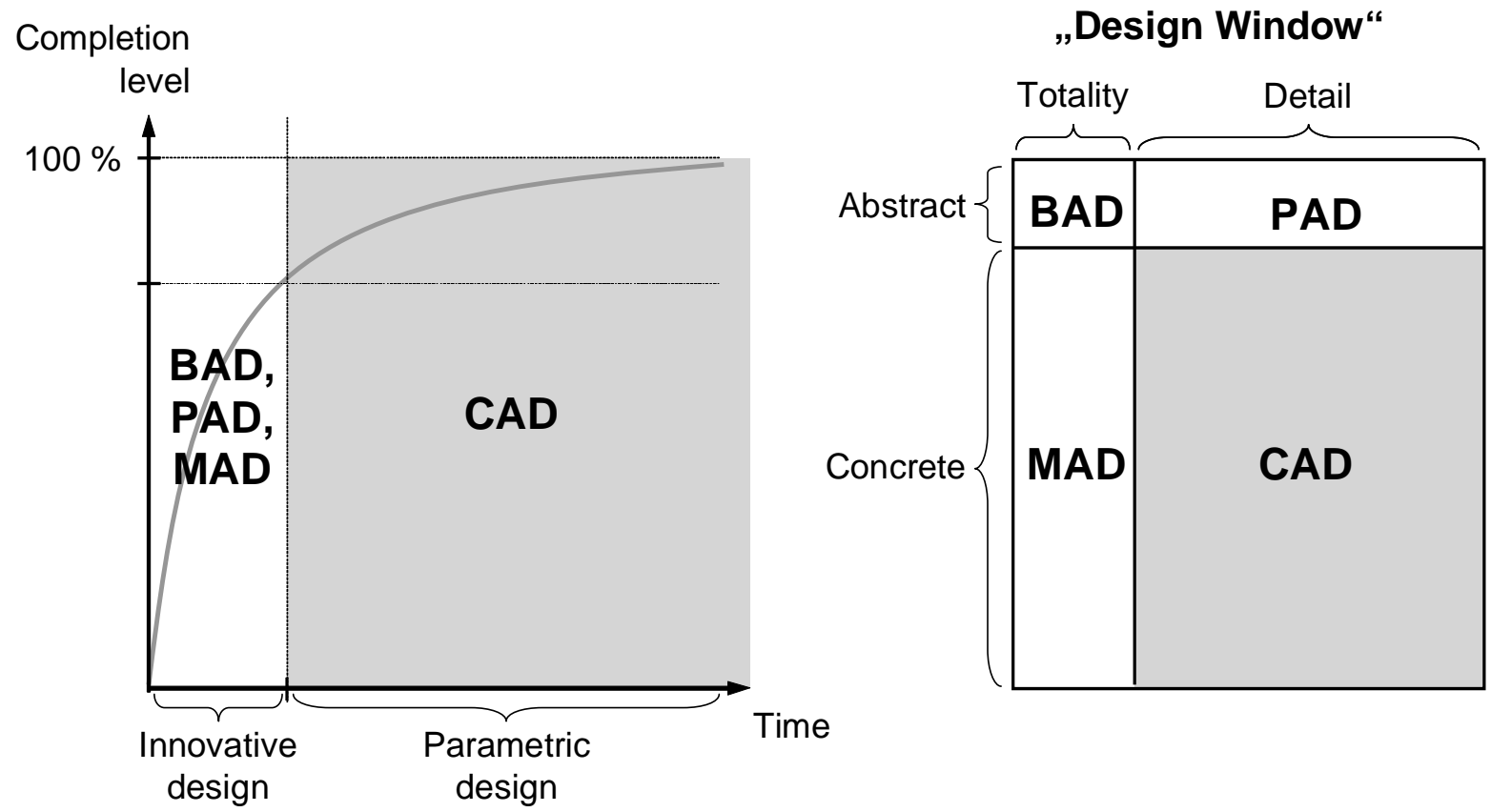

Figure 3-3: Application of different tools in Design according to OTTOSON (2003) with BAD = Brain Aided Design, $P A D=$ Pencil Aided Design, MAD = Model Aided Design, $C A D=$ Computer Aided Design

CAD is an integrative element of virtual product development (SPUR \& KRAUSE 1997), which is a technology-oriented framework for design tools. Elements of virtual product development are (SPUR \& KRAUSE 1997, p. 48):

- systems for geometric modeling

- systems for processing of features

- systems for graphical representation

- data management systems

- simulation systems

- knowledge-based systems 
Within the environment of CAD, there are other computer-based tools for design. These systems are often summed up by the expression CAx (in some literature, CAx is wittily called "Computer Aided Everything"; see CONAWAY 1995 for instance). Examples for such systems are:

- Finite-Element-Analysis (FEA),

- Computer Aided Styling (CAS),

- Computer Aided Manufacturing (CAM),

- Computer Aided Quality Control (CAQ),

- Product Data Management (PDM),

- Computer Aided Industrial Design (CAID),

- Computer Aided Production Engineering (CAPE),

- Computer Aided Testing (CAT)

- Rapid Prototyping (RP)

- Virtual-Reality-Simulation (VR)

CAD itself provides a number of "tool-like" advantages beyond pure representation of the product description (EVERSHEIM et al. 1996); which are for example:

- collision control in assemblies

- kinematical analysis

- simulation of production processes

- automated generation of models for FEA (Finite Element Analysis)

- availability of exact data for production

- integration of Rapid Prototyping

- ...

\subsubsection{Rapid Prototyping}

Rapid Prototyping (RP) is a technology that automatically creates of physical models based on 3D-CAD-data in a short amount of time. At the present time, there are at least eight different Rapid Prototyping procedures that may be used efficiently and effectively in mechanical engineering design (FÄHRER 2002). The resulting models may strongly differ in achievable accuracy, possible size and variety of usable material, as well as in the resulting strength and durability of the model. For that reason, it is not easy to choose the procedure and the point of time within a design process to apply such a model for a certain purpose. Internet portals may provide users with necessary information about the technology and corresponding service industries (e. g. RP-NET 2003 in Germany). 
The procedures can be fundamentally distinguished by the raw material that is used to build the physical model. The building material can be solid, liquid or in powder form. Common RP-procedures build the model stepwise in layers that are attached to each other. In the following paragraphs, for each of these kinds of procedure, one example is briefly described ${ }^{1}$ :

- Layer Object Manufacturing (LOM):

LOM uses paper, plastics, metals or ceramics in the form of sheets as building materials. The basic components of a LOM-machine are a component platform, a $\mathrm{CO}_{2}$-laser, a 2D-plotter mechanism, a heatable press roll and an equipment to supply sheet building material. The model is built up on the platform. For this purpose the $\mathrm{CO}_{2}$-laser continuously cuts the film building material along the components contour. Thereby the laser is directed by a computer-controlled 2D-plotter mechanism. The layers are interlinked by glue, which is applied to one side of the film and activated by a heated roll mechanism. After generation of a layer the component platform is lowered by a sheet thickness and covered with a new layer by the material supply.

- Fused Deposition Modelling (FDM):

The FDM-procedure uses AABS or wax building materials. The basic components of a FDM-machine are a component platform, two extrusion nozzles and a 2D-plotter mechanism. The model is built up on the platform. The thermoplastic material is heated up (until it is liquid) and extruded by a robotic nozzle according to the component geometry. Thereby the extrusion nozzle is directed by a computercontrolled 2D-plotter mechanism. After building a layer the component platform is lowered by a layer thickness and the process is repeated. When the material cools down and thereby hardens up, the layer becomes solid and connects top the layer underneath. It is necessary to generate support structures in correspondence with undercuts and cavities in order to prevent these areas from subsiding. The support material is applied by a second extrusion nozzle along with the building material and can be removed after the procedure.

- Selective Laser Sintering (SLS):

SLS uses nylon, waxes, polycarbonate or polystyrene in powder from as building material. Furthermore, synthetic coated metal powder (a special mixture of metals) or very fine-grained croning sand may be used. The SLS- machine consists of a construction platform, a wiping mechanism, a $\mathrm{CO}_{2}$-laser, a mirror galvanometer and a powder tank. The $\mathrm{CO}_{2}$-laser selectively cures the free surface of the powder which is evenly spread out on the platform. Thereby, the laser is directed by a computercontrolled mirror galvanometer. After exposure of a layer the platform is lowered by a predefined layer thickness into the powder tank. A wiper mechanism eases the application of a new powder layer. The laser penetrates so deep into the powder that the actual model layer as well as the previous layer underneath are partially smelted. When the smelted powder hardens subsequently, the particles within one layer

\footnotetext{
${ }^{1}$ The description of the procedure is adapted from RP-NET (2003)
} 
connect as well as the particles on top of each other. Therefore, no glue to connect the layers is necessary. Still, the particles are not smelted completely, so a subsequent post-curing of the model is required. The generation of support structure is not necessary since undercuts and cavities may rest on the surrounding powder sufficiently.

\subsubsection{Virtual Reality}

Virtual Reality (VR) is a computer-based technology that aims to represent "virtual worlds" as realistic as possible. Those worlds are usually called "scenarios" in which the user may perceive virtual objects and surroundings.

In the ideal case, VR distinguishes itself by the following aspects (ENCARNAÇAO 1995, pp. 592): Virtual Reality ...

- ... is interactive.

The user can manipulate virtual objects and surroundings.

- ... is intuitive.

Special input devices enable the user to manipulate the scenario as they might do in reality.

- ... works in real time.

The representation of the manipulated virtual objects happens without any perceivable time delay according to the input of the manipulation.

- ... is a multimedia application.

Due to special output devices, the representation of scenarios affects several human senses.

Most VR-systems take advantage of the visual sense of the user, which is probably the most important sense for the recognition of objects. In reality, most objects are spatial and usually they are seen in a 3-dimensional way. In the case of VR, a 3-dimensional visual perception is achieved by affecting the user's eyes separately. This principle is called stereo view. Figure 3-4 shows a schematical illustration of such a VR-system. The computer calculates the representation of an object for two slightly different points of view. Each representation matches the view from one of the user's eyes. With the help of certain data output devices, the different views are displayed only to the appropriate eye (view 1 and 2). This is usually achieved by certain kinds of goggles - we will specify these devices later on. Thereby, the user seems to perceive the object in a 3-dimensional way. Many VR-systems take into account that in reality, users do not only watch the surrounding world but also move within the world. Every motion of the user alters the angle and the distance of view on the surrounding objects. Some VR-systems determine the user's position with the help of socalled tracking-systems and accordingly modify the represented views on the virtual object. The tracking system usually localizes the position and the direction of the stereo view goggles for that reason (tracking system 1). 


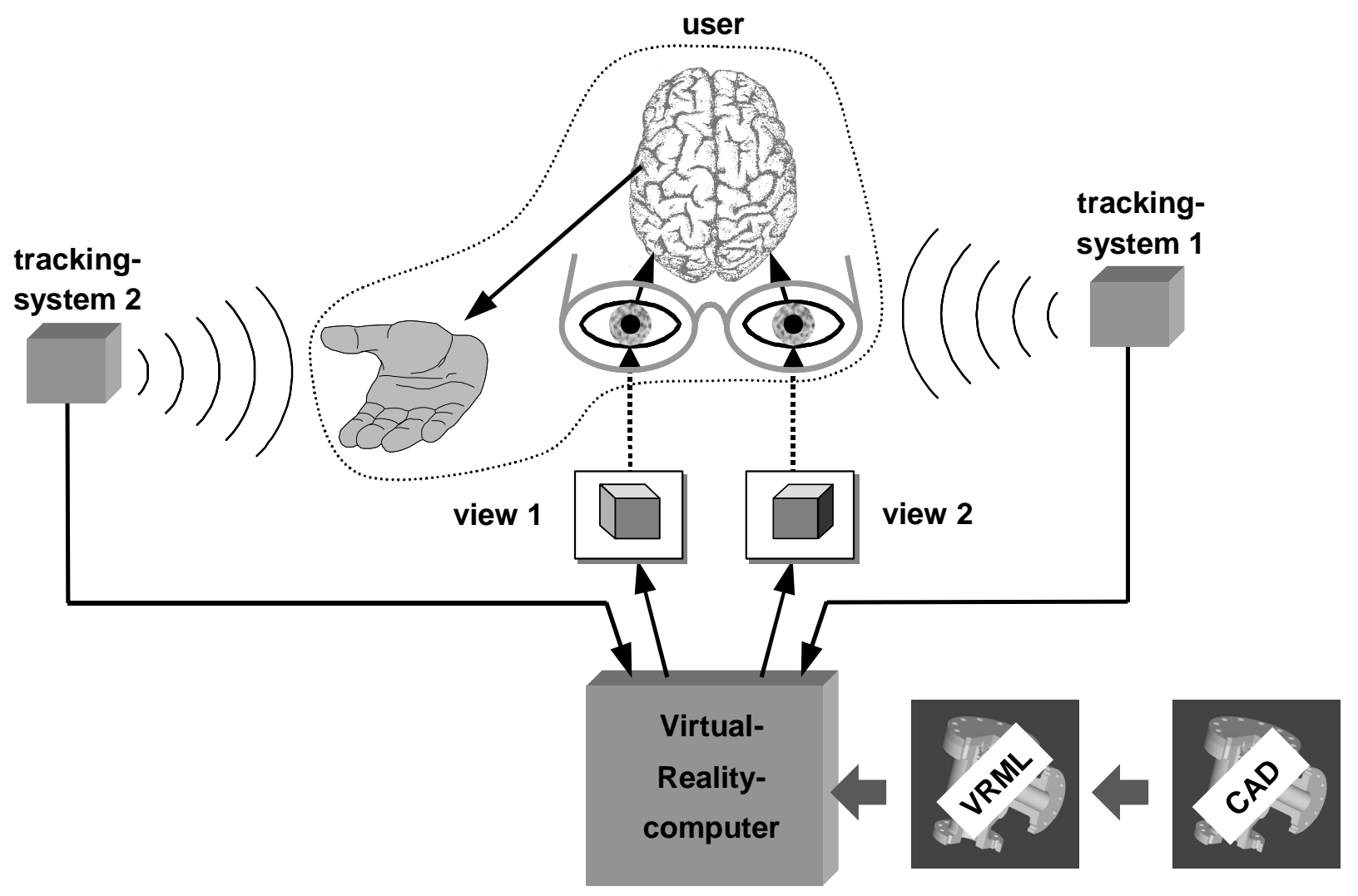

Figure 3-4: Schematical illustration of the functional principle of Virtual Reality

To achieve the user's ability to interact with virtual objects in an intuitive way, special devices for data input record the user's actions and gestures. Usually, the motion of the user's hand is tracked for that reason (tracking system 2). Some systems determine position and direction of the hand and even ascertain the posture of the fingers. A gesture that seemingly touches or grasps a virtual object is recognized and the representation of the object is altered in accordance to this virtual manipulation of the object.

A common data output device is the so-called shutter goggle that is worn by the user while watching any kind of display (such as a monitor). The display represents the two different stereo views in an alternating manner with a high frequency. The goggles are controlled by some kind of emitter and turn each of the user's eyes blind in the same alternating manner (usually by glasses that can be darkened). Therefore, each eye perceives the view with such a high frequency that the alternation can hardly be sensed. Hence, the represented object appears to be truly 3-dimensional. Another possibility to achieve a stereo view is the use of a Head-Mounted-Display (HMD). This device places miniature monitors right in front of each of the user's eyes. Each of these monitors constantly displays a specific view such that the object is perceived in 3-dimensions (see Figure 3-5). 


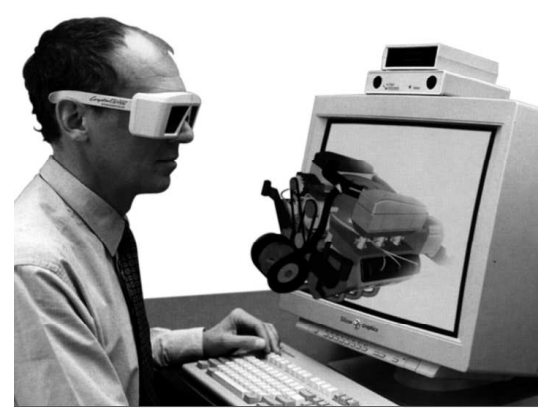

shutter goggles with monitor

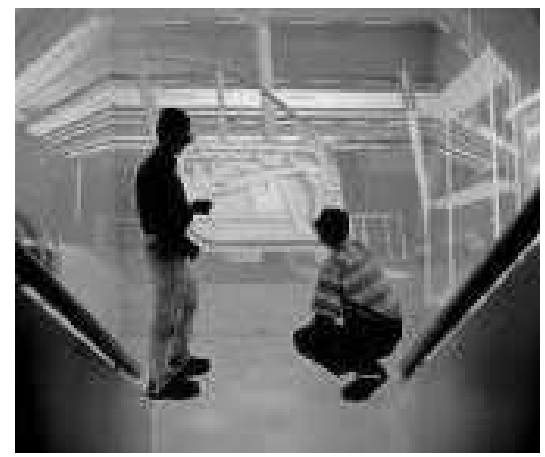

cave

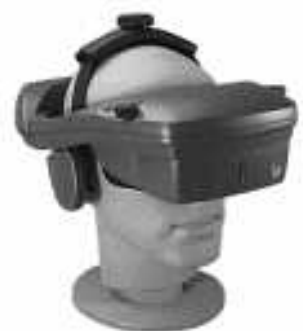

Head-Mounted

Display (HMD)

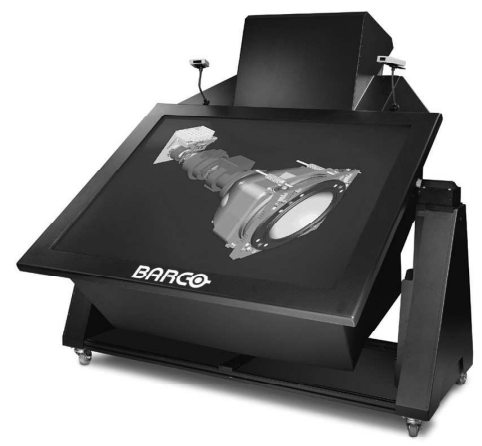

workbench

Figure 3-5: Common data output systems for Virtual Reality

The previous consideration of output devices concerns another characteristic that is associated with VR and this is immersion. An HMD allows for complete immersion in a virtual world; no information from the real world is (visually) perceived by the user anymore. Nearly the same applies to a cave, which is a room with walls that serve as projection screens for the virtual scenario. The user needs to wear shutter goggles that provide stereo view (as described above) and seems to be surrounded by the scenario. A strong feeling of immersion is created, but the user can still see his own body. By contrast, watching a monitor via shutter goggles allows watching the virtual world "through a window", but the real world that surrounds this window is still there. In this case, the user only becomes partially immersed into a virtual scenario (the size of the window may vary - a workbench is actually a huge monitor shaped as a table). MiLgRAM \& KISHINO (1994) describe the combination of real and virtual aspects in VR by the Reality-Virtuality-Continuum that is shown in Figure 3-6. While Mixed Reality (MR) describes the whole range between reality and virtuality, Augmented Reality (AR) consists of more real components than virtual ones. Accordingly, Augmented Virtuality (AV) 
consists of more virtual components. We will use VR as a generic term for the whole idea and technology respectively that enables this continuum.

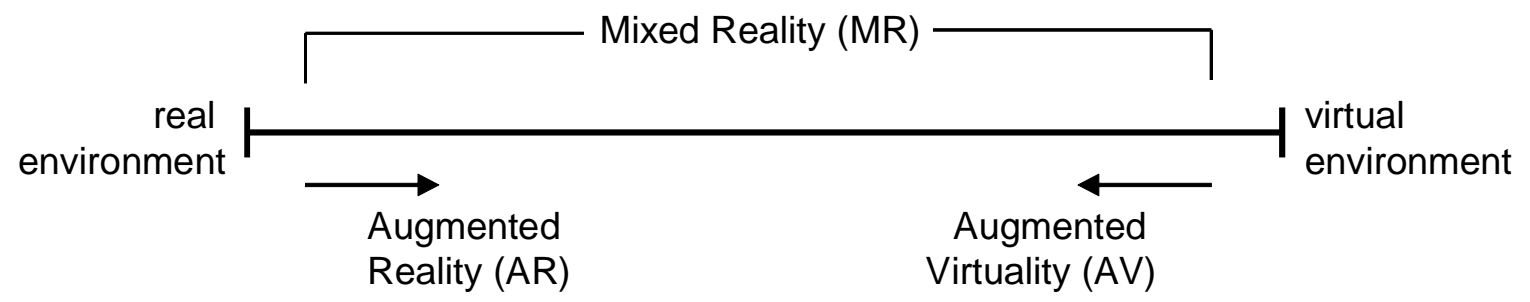

Figure 3-6: Reality-Virtuality-Continuum according to MILGRAM \& KISHINO (1994)

Examples for VR-input devices are the space mouse and the data glove. The space mouse provides a grasp that can be moved in the direction of three translatory and three rotational degrees of freedom. Thereby, a virtual object can be shifted and rotated in space. However, a data glove allows for manipulation that goes beyond shifting and rotating. The glove is localized in space by a tracking system and moreover, sensors that are integrated in the glove determine the posture of the fingers. The computer can integrate the user's hand completely into the VR-scenario and therefore, manipulation that equals the process of actually grasping an object may be achieved (see Figure 3-7).
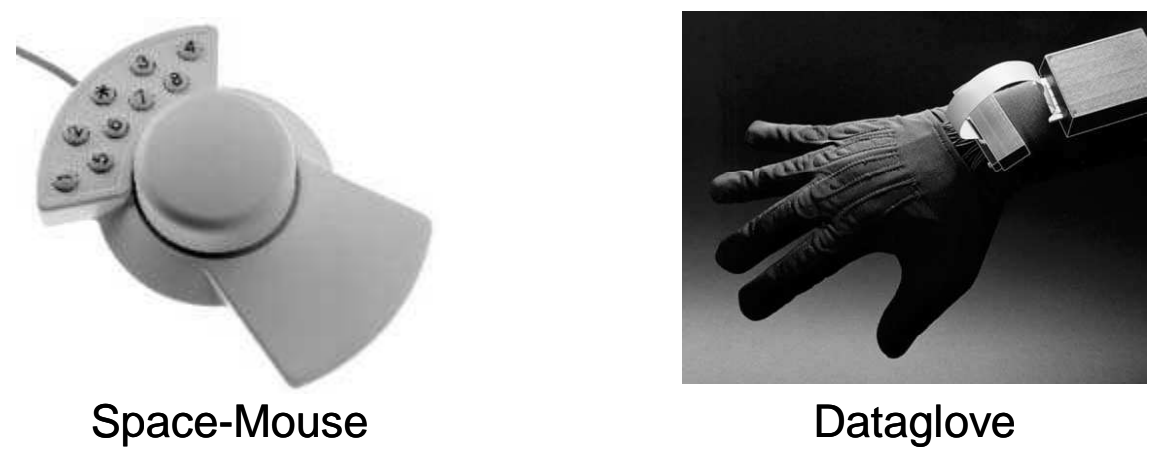

Figure 3-7: Common data input devices for Virtual Reality

In engineering design, VR-scenarios may be used for several kinds of simulations, such as the representation of product properties concerning assembling, ergonomics and industrial design. Future products and components can be represented in a realistic way without the need to actually manufacture a prototype. VR-scenarios are usually based on VRML ${ }^{1}$-data, which can be derived from CAD-data.

\footnotetext{
${ }^{1}$ VRML = Virtual Reality Modelling Language
} 
A basic problem of most VR-simulations that require the intuitive interaction of the user (such as the simulation of an assembling process) is the absence of tactile, haptic and force feedback from virtual objects. At first, objects are visually perceived by the user but as soon as he tries to grasp the object with the help of a data-glove, he cannot feel the object. This may distinctly spoil the impression of seemingly real objects. For this matter, special "feedback" devices are being developed (see Figure 3-8), such as a glove with an exoskeleton that may inhibit the finger's motion (top left). Thereby, the user cannot close his hand as soon as he grasps an object in virtuality and he receives a haptic feedback from this virtual object. Moreover, there are devices that aim to give a tactile feedback to the user. A so-called tactile display may apply pressure to distinct regions of the user's fingertip (top right). Thereby, he may be able to feel virtual surfaces and edges while stroking a virtual object with his fingertips.

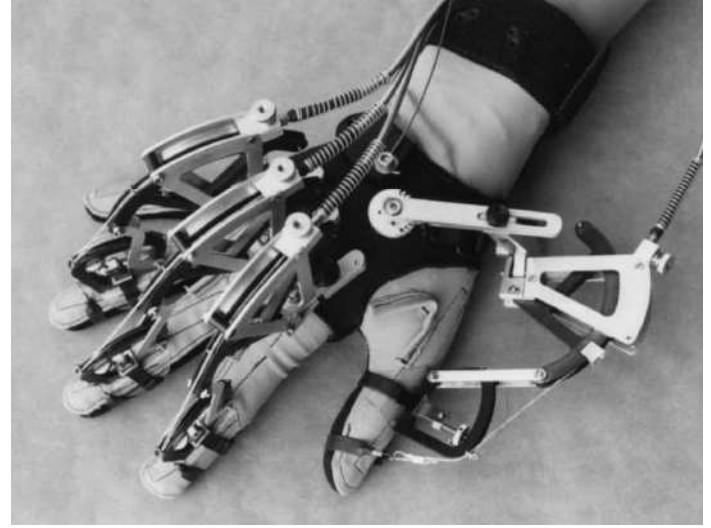

haptic feedback

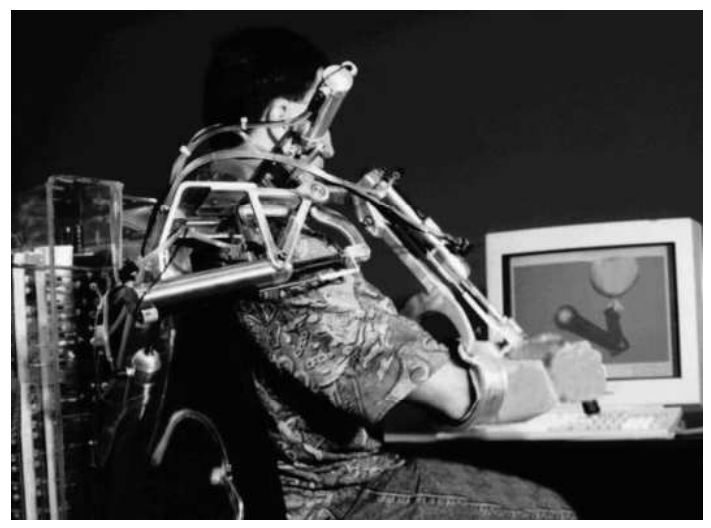

force feedback

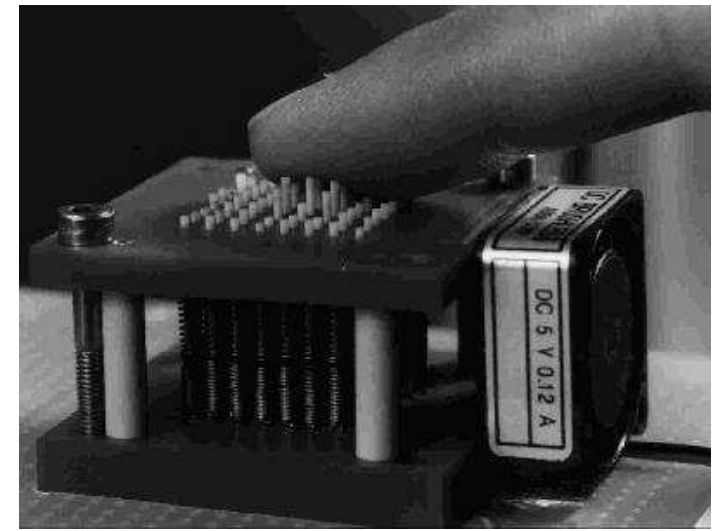

tactile feedback

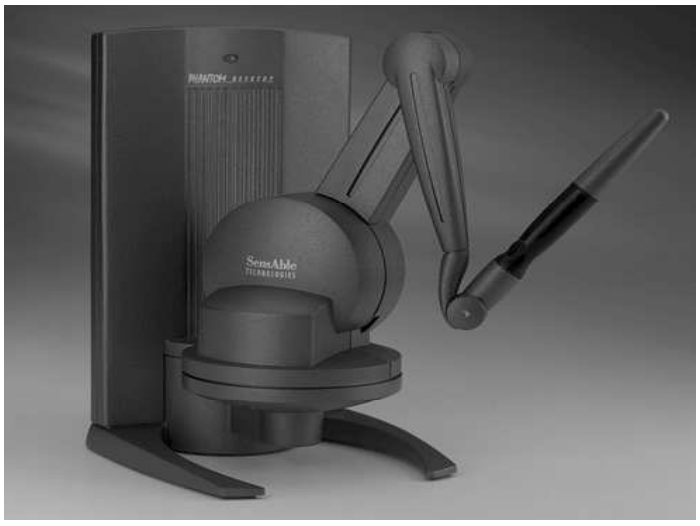

force feedback

(Phantom Desktop)

Figure 3-8: Feedback devices for Virtual Reality 
Still, with the help of these devices, the user cannot sense the weight and the inertia of an object he interacts with in virtuality. Force feedback devices for that matter would not only be attached to the user, but they would need to prop up against the surrounding as well. They may apply an outer force to the user and thereby give the impression weight and inertia. Such devices may distinctly restrict the user in his freedom of action (bottom left). An example for a relatively simple force-feedback device is the Phantom Desktop (bottom right). It consists of a handle that is connected to a stand by a system of levers. Each joint between the levers contains a servo motors that may block up or move the joint. The user can grasp the handle and either tap it against objects in virtuality or slide the handle along the surface of virtual objects. The servo motors are controlled in such a way that the handle is prevented from penetrating the virtual object and thereby, the user seems to feel the object through the reaction force of the handle.

In summary it may be said that VR seems to be an appropriate gateway for accessing any kind of future digital world. However, the more precisely this world tries to copy reality, the more costly it is to provide a persuasive representation of that world. The next chapter will deal with the use of VR (among other media and tools) for simulation purposes in engineering design. Thereby, we need to keep in mind that VR may still require a great deal of development to fulfil those functions that are already being demanded from this technology.

Nevertheless, it will turn out later on that VR may already serve for purposes other than those that are considered in the following chapter. This is because simple, but highly abstract VRrepresentations may be achieved rather easily and still be very useful in design. That remains to be seen in chapter 5 , though.

\subsection{Actual Use of Media and Tools for Design in Mechanical Engineering}

This thesis aims to lay an empirical and scientific foundation for improvement of design media and tools used in industry. Therefore, we need to investigate the benefits and deficits of common design media and we need to know the basic demands and wishes from designers according to their daily work. With the help of two surveys among designers in mechanical engineering, we will give answers to the following questions:

- What are the characteristics of design tasks that designers usually work on?

- Which are the most common media and tools that are actually used by designers?

- In which way do the designers use those media and tools?

- For what purposes do designers use those media and tools?

- What are the effects of the use of media and tools?

- How satisfied are designers with their media and tools?

The first survey mainly investigated the use of sketches and physical models. The questionnaire was sent to German-speaking designers from various branches including 
mechanical and facility engineering, electrical engineering, automotive engineering, process engineering, precision engineering, textile machines, photo technology and industrial design. The questionnaire was completed by 107 designers. The sample mainly consists of designers who have attended an advanced technical college (39\%) or a university (37\%). $11 \%$ have additionally completed a doctorate study. The designers have an average working experience of about ten years, although it actually ranges from half a year to 43 years.

The second survey was carried out by the use of a digital questionnaire that was sent out via email or was downloaded from a website that was publicly accessible. It investigated the use of CAD in relation to other media and tools, such as sketches, Rapid Prototyping and Virtual Reality. Completed questionnaires were received from 65 designers mostly working in the domains mechanical engineering, facility engineering and automotive industry. The work experience of the designers again averages ten years, ranging from one to 42 years. In average, the designers work with $\mathrm{CAD}$ in general for eight years and work with the actual system for 5 years. 3D-CAD-systems are used by $87 \%$ of the designers, while $48 \%$ use a 2D-system (multiple answers were allowed). FEM is available to $55 \%$ of the designers, rapid prototyping is available to $34 \%$ and only $17 \%$ have the opportunity to use virtual reality.

In general, both samples of designers may be regarded as representative cross sections. In the following chapters, we will mainly view results from second survey referring to design tasks in general and to the explicit use of sketches and CAD. If results from the first survey are presented, then they are labeled by the index (1). In both surveys, two types of questions were asked. According to the subject, either several items with suggested answers were given that could be chosen and individually supplemented, or a range from "completely true" to "not at all true" (respectively "always" to "never") was given. The actual formulation of each question is indicated by a footnote. For further details of the procedure used to carry out the first survey see RöMER et al. (2001). The second survey was carried out basically in the same way (except for the differences described above).

\subsubsection{Characteristics of Design Tasks in Mechanical Engineering}

Most of the designers usually work on tasks that involve theories of classical mechanics (91\%). Furthermore, about a quarter of the tasks involve aspects from industrial design $(26 \%)$, ergonomics $(25 \%)$ and fluid mechanics $(23 \%)$. Fewer tasks require knowledge from electrical engineering and thermodynamics (each $15 \%$ ). Those fields of knowledge suggest certain characteristics of the product to be designed. When theories from mechanics are involved, then it may be expected that designers need to pay attention to product attributes that depend on force and motion. Therefore, the designers were asked to choose statements according to the product attributes that are most essential for the design tasks. The results for this question are presented in Figure 3-9. 


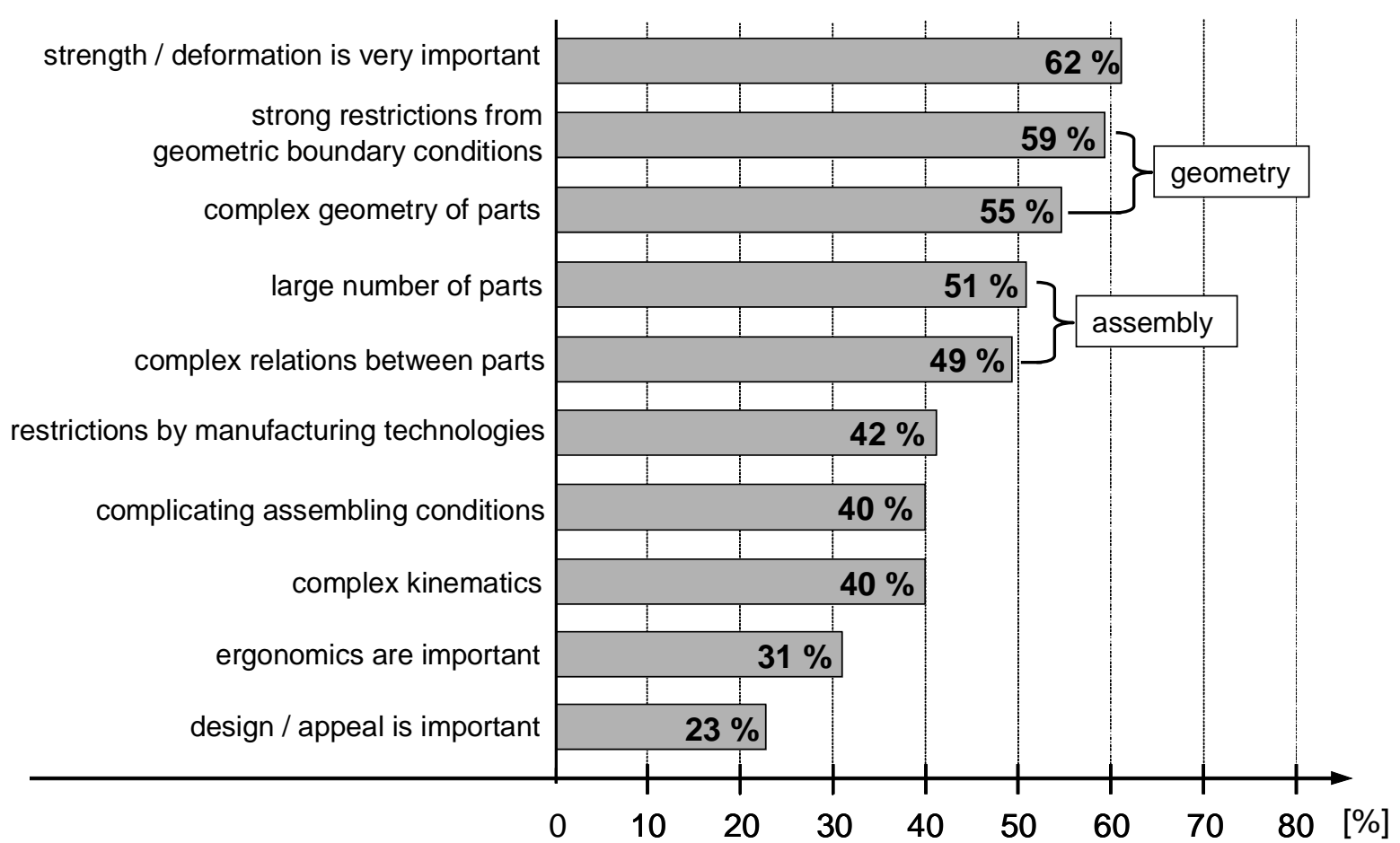

Figure 3-9: Characteristics of the design tasks usually processed by the designers ${ }^{1}$

The importance of the strength and deformation behavior of parts is seen as a main characteristic of design tasks by a majority of the designers (62\%). It is the task characteristic that is named most often. Additionally, strong restrictions from geometric boundary conditions $(59 \%)$ and the complexity of the geometry of parts $(55 \%)$ were named. Both the latter statements can be summarized as aspects of "pure" geometry. They are followed by aspects that refer to assembly characteristics: The large number of parts within an assembly group $(51 \%)$ and the complexity of relations between parts (49\%). Strong restrictions from processes of manufacturing (42\%), assembling (40\%) and complex kinematics (40\%) are mentioned less often, but still seem to be relevant.

In the case of most engineering disciplines, those attributes of a product which are constituted directly by the designer are shape, dimension and material (EHRLENSPIEL 2003, pp. 24). Aspects of manufacturing are usually specified in the second place. The product usually consists of an assembly of parts, so the spatial arrangement of the parts as well as the interconnections between them is directly constituted by the designer. In turn, the designer also has to specify the process of assembling.

Other attributes of the product are determined indirectly by the designer - they are a consequence of the combination of attributes he constituted directly (LINDEMANN 2003B).

\footnotetext{
${ }^{1}$ Question: "Which characteristic(s) do a design task that you usually work on describe at best?"
} 
Such attributes describe the behavior of the product. For example, a designer cannot directly specify the power of an engine. But he may directly specify such attributes as the stroke and the diameter of a cylinder along with the number of cylinders (along with many, many other direct attributes in fact). With the help of these specifications, he tries to cause a certain power, yet he cannot directly constitute it. Indirect attributes result from direct attributes according to logical, physical and technical principles.

Figure 3-10 outlines this matter. A product definition may be seen as a jigsaw puzzle in which the pieces are the direct attributes. The combination of different pieces of the puzzle determines the value of a certain indirect attribute. Still, the pieces have to fit together in order to form a consistent product that is producible. In general, the abstractness of the product description that the designer works on determines which attributes are direct and which ones are indirect. On a very abstract, functional level, single functions can be constituted directly by the designer. In the further course of design, those functions may turn into indirect attributes that need to be achieved by material, geometry, etc.

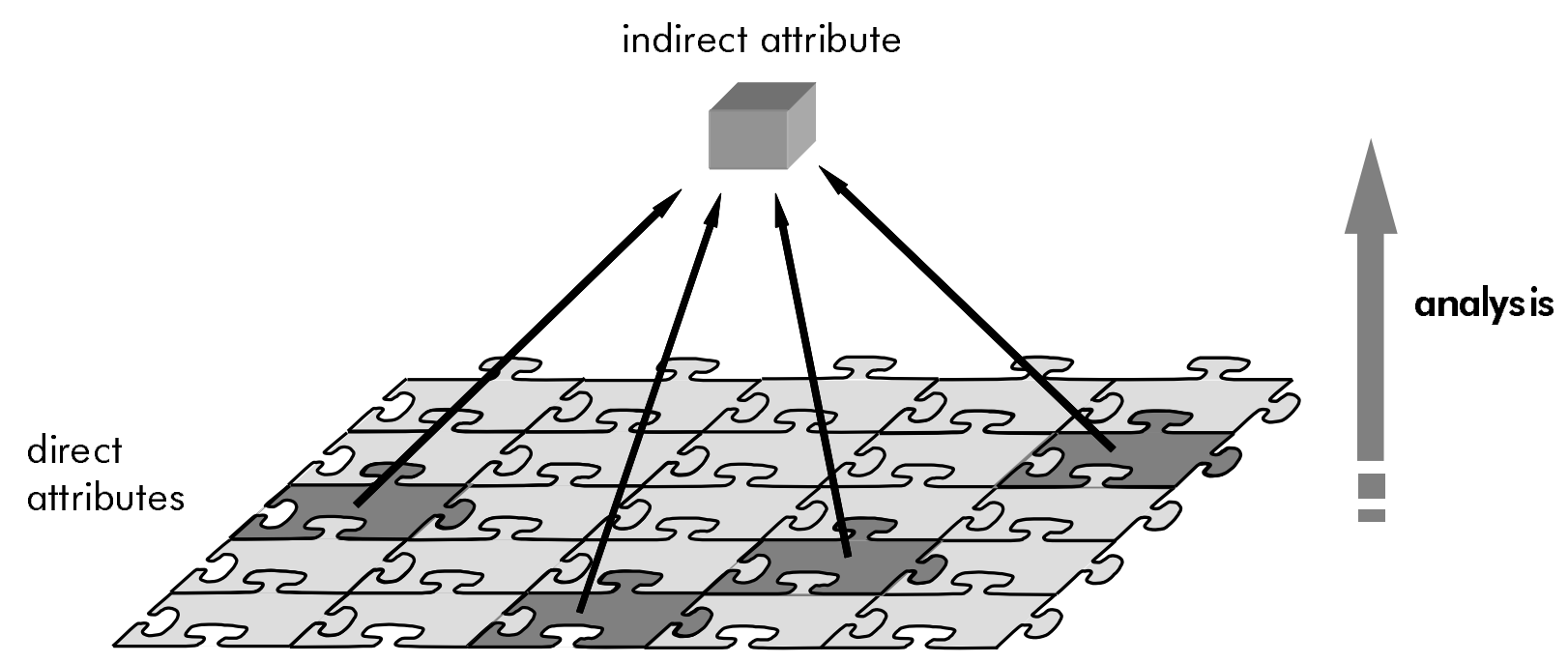

Figure 3-10: Connection between direct and indirect product attributes

Requirements on a future product usually refer to its desired behavior and its indirect attributes respectively (EHRLENSPIEL 2003, p. 25). For different products, there are different indirect attributes that need to be determined in order to evaluate the success of a design process. The checklist for drawing up a requirement list from PAHL \& BEITZ (1996, p. 133) provides a great number of possible indirect attributes of a product (see Figure 3-11). This list summarizes examples of product attributes into groups that refer to certain superordinate classes such as geometry and kinematics. Let us now have a brief look at both these two classes. 


\begin{tabular}{|c|c|}
\hline Geometry & $\begin{array}{l}\text { height, length, thickness, diameter, need for space, number, structure, } \\
\text { connection, enlargement }\end{array}$ \\
\hline Kinematics & kind of motion, direction of motion, speed, acceleration \\
\hline Forces & $\begin{array}{l}\text { amount, direction, alternation in time, weight, load, deformation, stiffness, } \\
\text { flexibility, stability, resonance }\end{array}$ \\
\hline Energy & $\begin{array}{l}\text { power, efficiency, loss, friction, ventilation, pressure, temperature, humidity, } \\
\text { heating, cooling, storage, energy transformation }\end{array}$ \\
\hline Material & $\begin{array}{l}\text { physical, chemical and biological properties, standards, flow of material, } \\
\text { transport of material, logistics }\end{array}$ \\
\hline Signal & input, output, display mode, control system, monitoring system \\
\hline Safety & active and passive safety system, protection, environmental safety \\
\hline Ergonomics & man-machine-interface, operation, shape, clearness, lighting, manuals, \\
\hline Production & $\begin{array}{l}\text { restrictions by the production plant, preferred method of production, largest } \\
\text { producible dimensions, attainable quality and tolerances }\end{array}$ \\
\hline Controlling & available controlling systems, standards and restrictions (ISO, DIN, ASME, ...) \\
\hline Assembly & installation, dismantling, ergonomics, standards \\
\hline Transportation & Restrictions by cranes, maximum size and weight, transportation charges \\
\hline Use & $\begin{array}{l}\text { noise, attrition, effects from the environment (humidity, temperature, weather } \\
\text { conditions) }\end{array}$ \\
\hline Maintenance & $\begin{array}{l}\text { Maintenance-freedom, maintenance cycles, inspection, replacement, duration } \\
\text { of maintenance }\end{array}$ \\
\hline Recycling & reuse, recycling, dismantling, devastation, wrecking, storage, disposal \\
\hline Costs & $\begin{array}{l}\text { life cycle costs (development, production, assembly, use, ...), investment, } \\
\text { amortization }\end{array}$ \\
\hline Time & milestones, deadlines, date of delivery, effects from time pressure \\
\hline
\end{tabular}

Figure 3-11: Checklist for drawing up a requirement list (PAHL \& BEITZ 1996, p. 133)

The class geometry contains all kinds of dimensions, which are direct attributes that can be constituted by the designer. Still, the need for space may be an indirect attribute if the product is an assembly. Need for space is a consequence of the way that each part was designed and the way these parts are connected. The logical principle that determines the overall dimension of an assembly is the "simple" addition of each part's dimension. 
The direction of a part's motion within an assembly may be even harder to determine. This may be exemplified by the design of a mechanism for the foldable roof of a convertible automobile that took place at the institute of product development at the Technische Universität München (STETTER \& PACHE 1998). The frame of a convertible's roof often consists of three linkages that are coupled to each other. Each linkage consists of four bars that are connected by four joints. A requirement within this design process was to achieve a defined line of motion for the endpoint of the frame during operation. This was necessary to allow for an easy-to-use closure between the frame and the windshield. Slight changes to the frame's geometry caused an alteration of the motion line that was nearly unpredictable for the designers. For that reason, the designers built a simple physical model of one four-bar linkage in order to better understand the relation between design changes and alterations of the motion line. The principles of mechanics provide this relation, but the designers still could not constitute the motion line directly.

\subsubsection{Use of Common Media and Tools in Mechanical Engineering}

Graphical, physical and digital models (EHRLENSPIEL et al. 2003) were regarded as possible design media for conceptual design in the first survey. The results show that CAD and freehand sketches are the most common design media for conceptual design and task clarification $^{1}$ (see Figure 3-12). Sketches and CAD are used significantly more often than drawings that are true to scale and (simple and complex) physical models.

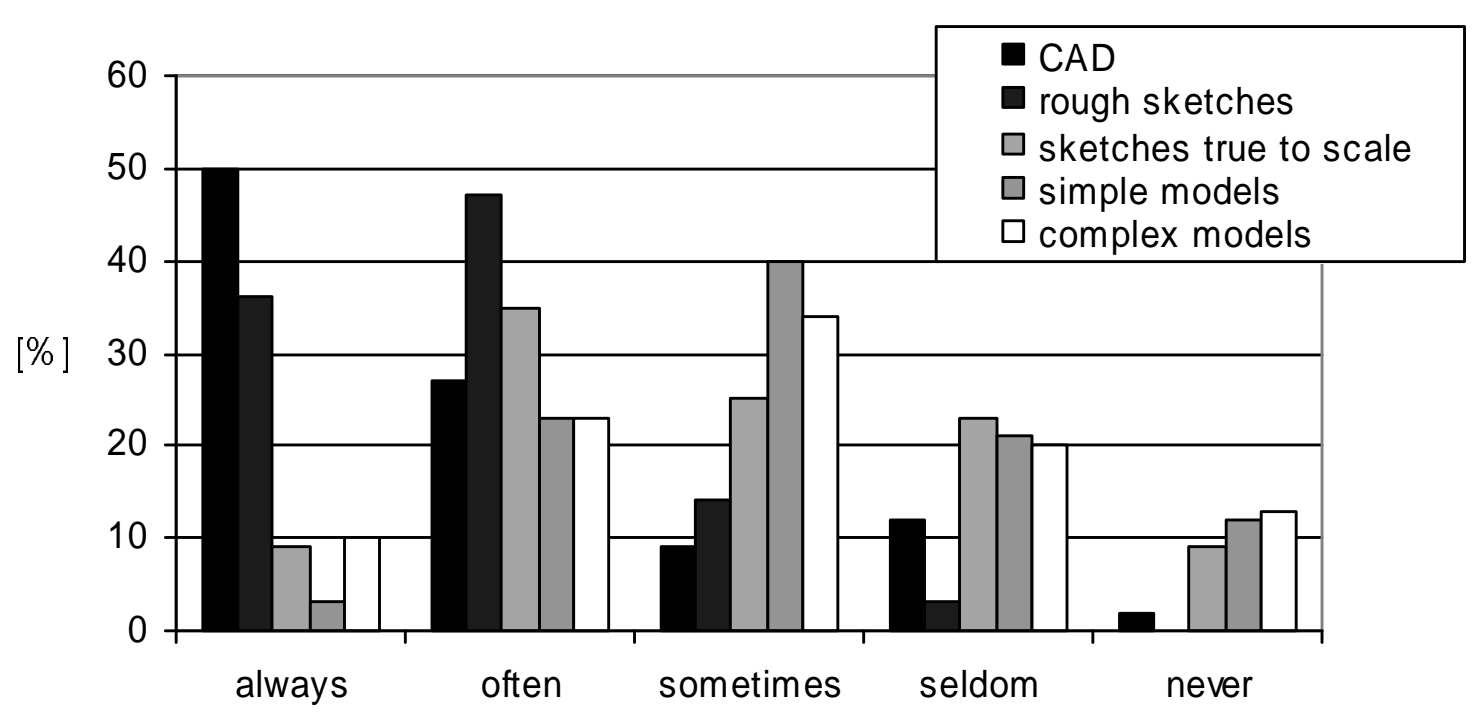

Figure 3-12: Frequency of use of design media during conceptual design and task clarification ${ }^{2}$ (1)

\footnotetext{
${ }^{1}$ This question item does not differentiate between task clarification and conceptual design. It was assumed that both these design activities can be regarded together as the early phase of the design process.

${ }^{2}$ Question: "How often do you use ... for clarification of the task and for development of conceptual solutions?"
} 
Sketches and CAD are media that are not used independently from each other. More than half of the designers $(62 \%)$ stated that they use sketches "always" or "often" as a preparation for CAD work. Designers use sketches less frequently during CAD work, but still more than half of the designers $(63 \%)$ sketch parallel to CAD at least "sometimes." And $65 \%$ of the designers at least "sometimes" plot the actual CAD-model and draw within this plot (see Figure 3-13).

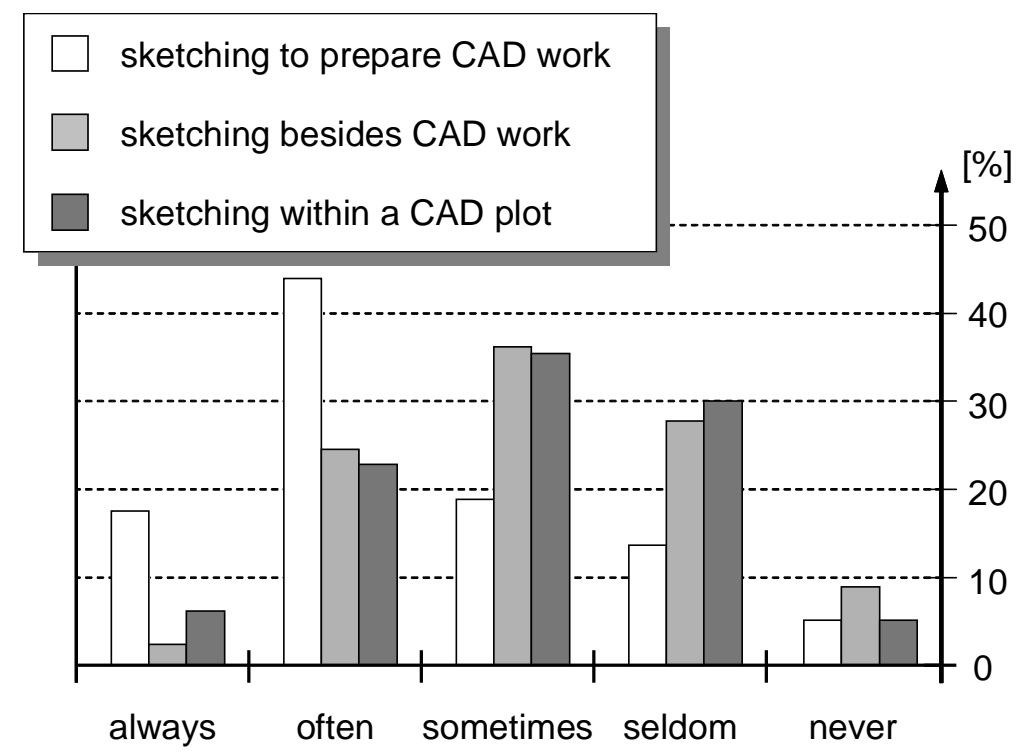

Figure 3-13: Frequency of use of sketches before and during CAD work ${ }^{l}$

The results presented above show that there is a certain need for some designers to supplement their CAD work with the help of sketches. This corresponds with statements in literature, such as Fish (1994), who suggests that "CAD-systems make it hard to be vague" and therefore designers use sketches despite of CAD-systems. To assess the capabilities and most of all the drawbacks in actual CAD-systems, it is most interesting to clarify in which way and for what reason such a supplementation takes place.

In the following considerations, we will focus on CAD and sketches as the most dominant media for conceptual design. Still, we will regard rapid prototyping and virtual reality systems as possible supplementations.

\footnotetext{
${ }^{1}$ Questions: "How often do you draw sketches especially to prepare CAD work?"

"How often do you draw sketches during CAD work?"

"How often do you sketch within a plot of the actual CAD model?"
} 


\subsubsection{Specific Application of CAD and Sketches to Conceptual Design}

The designers were asked questions according to the intentions for the use of CAD (see Figure 3-14). Almost all the categories given in the questionnaire were chosen by at least a third of the designers. Only the use of CAD as a short-term reminder was chosen by just $9 \%$ of the designers. Besides communication support (89\%) and presentation (86\%), the concretion of solutions was named as one of the main intentions for the use of CAD (85\%). Still, only about half of the designers develop conceptual solutions with the help of CAD (49\%), although this may be seen as a major activity in any design process. BOLTE (1998) points out that CAD-systems may bring about drawbacks and weaknesses when certain design actions are carried out. STACEY \& ECKERT (1999) argue that CAD-systems may provoke bias in design procedure that may obstruct designers.

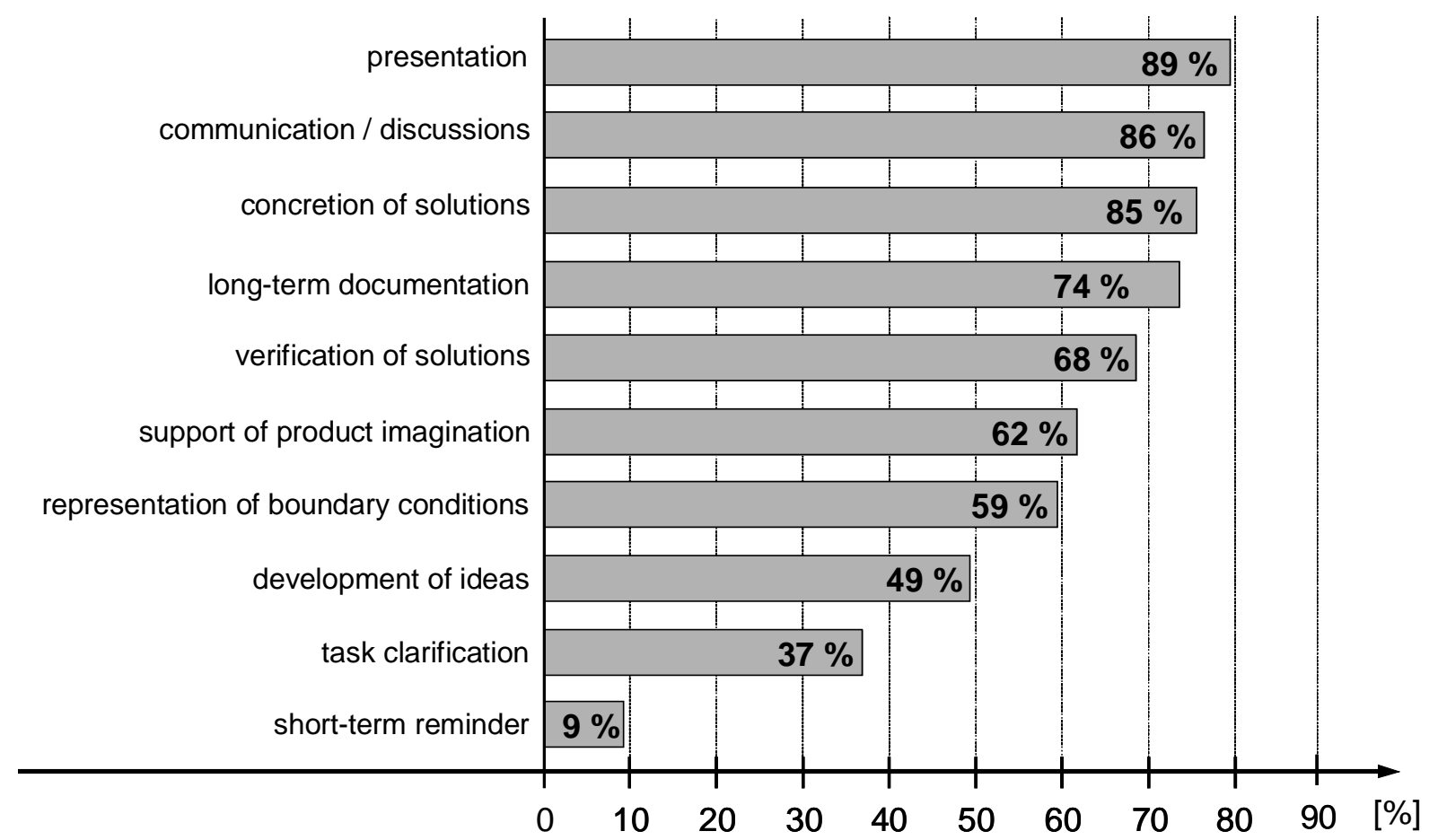

Figure 3-14: Intentions for the use of $C A D^{l}$

Since sketches are used parallel to CAD, it may be supposed that sketches serve as functional supplementation. Sketches that are used in parallel to CAD (see Figure 3-15) are mainly used for the development of ideas $(71 \%)$ and for communication purpose $(66 \%)$. Beyond that, sketches are also used as short-term reminders (54\%) and for further concretion of conceptual solutions $(55 \%)$. All in all, it is remarkable that the profile of design actions processed with the help of sketches clearly differs from the profile of actions processed with the help of

\footnotetext{
${ }^{1}$ Question: "For what purposes do you use CAD-models?"
} 
CAD. This is most noticeable in the case of idea development; it seems as if the most important benefit from sketches arises due to this function.

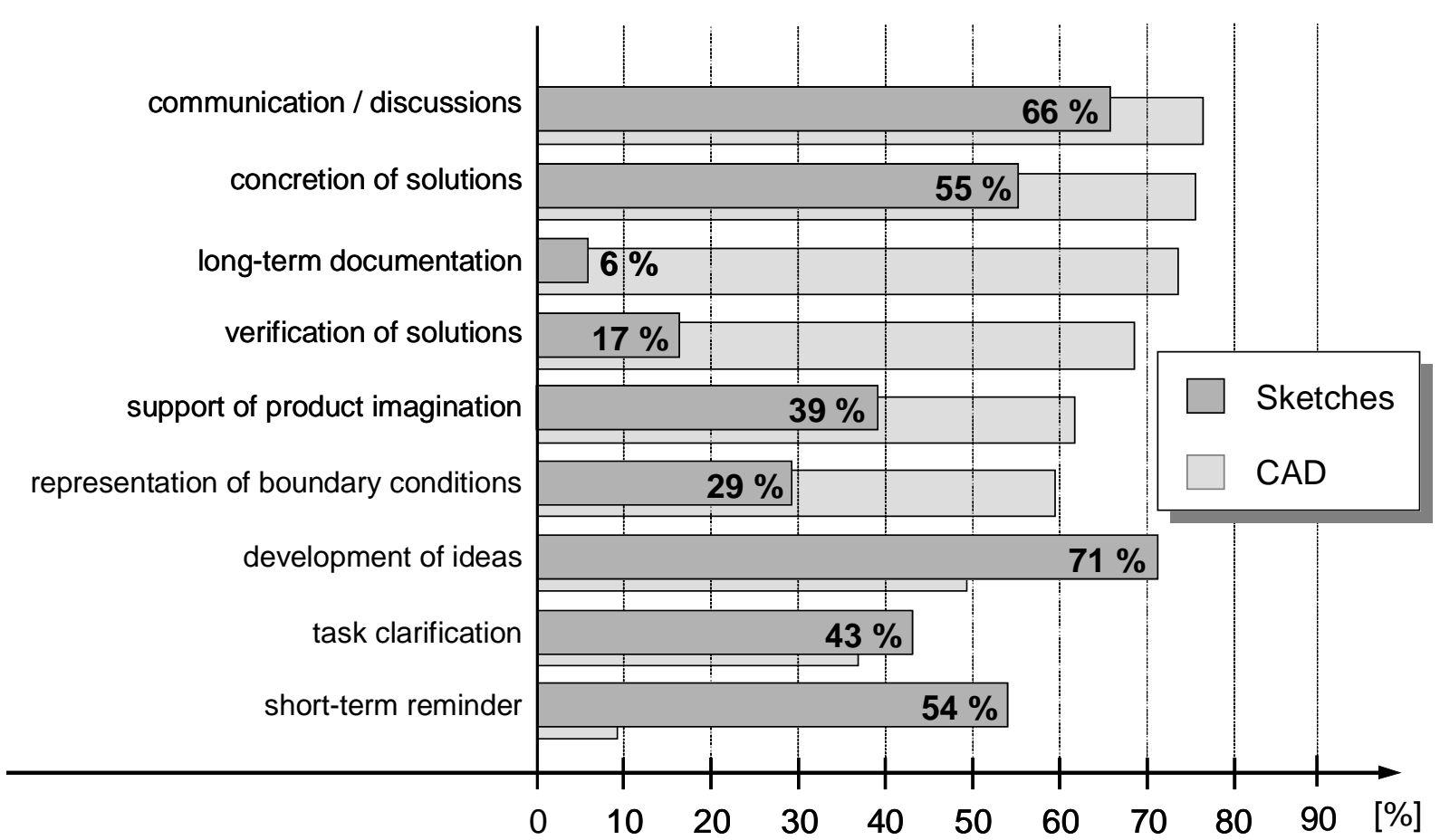

Figure 3-15: Intentions for the use of sketches during CAD work ${ }^{1}$ (in comparison with CAD - see Figure 3-14)

We have seen that CAD is not used in the same extent for all design actions, but may be supplemented by the use of sketches. In such a case, a sketch may serve as a tool providing the possibility for additional design action. Furthermore, it is possible that sketches are also used to represent certain product attributes that cannot be represented by CAD.

Going back to the results according to the characteristics of design tasks once more (see Figure 3-9), it shows that there are some indirect product attributes that need to be considered intensely during typical design processes. These are:

- material behavior (strength and deformation)

- geometric attributes

- assembly attributes

- attributes according to the manufacturing process

- attributes according to the assembling process

- kinematic behavior

\footnotetext{
${ }^{1}$ Question: "For what do you use sketches in parallel with CAD?"
} 
Strength and deformation are attributes that are "classically" analyzed with the help of FEMsystems, but the product features determining this behavior are predefined by CAD-systems. Since FEM is a rather time consuming tool and requires CAD-data as an input, it needs to be investigated how designers achieve certain strength and deformation attributes when designing via CAD. A similar question arises when kinematic behavior is concerned. This attribute is usually analyzed by simulation tools which again need CAD-data as input.

The designers were asked to judge the possibility of ascertaining specific product attributes directly from CAD models. There was a 3-point rating scale available ranging from "good" over "mediocre" to "bad". An additional category named "not relevant" could be chosen if an attribute does not apply to the products that the designer works on. The results are shown in Figure 3-16 (the category "not relevant" is not charted).

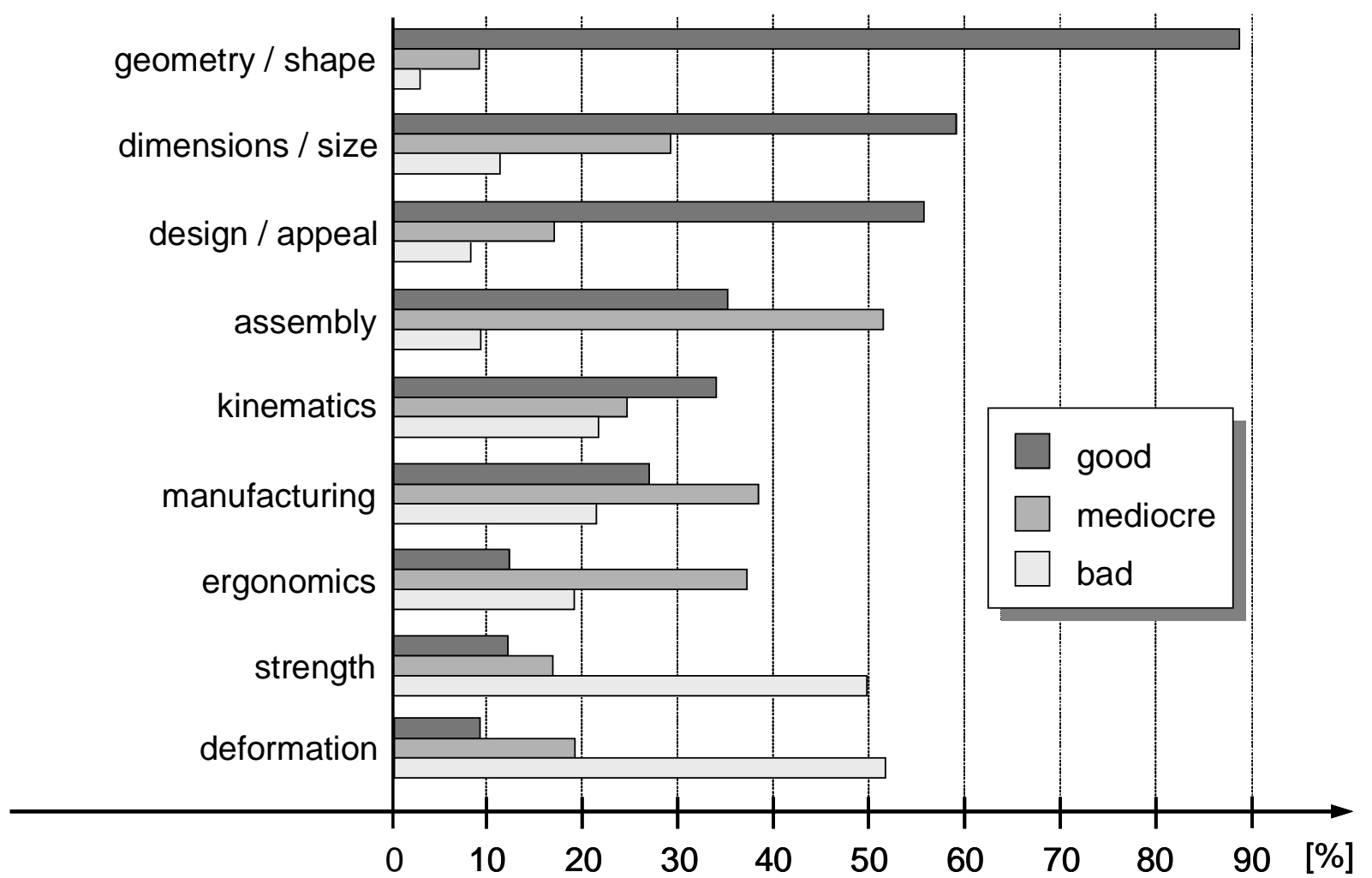

Figure 3-16: Possibility to ascertain specific product attributes with the help of CAD models ${ }^{l}$

With the help of CAD, the designers seem to be capable of recognizing the relevant geometry and shape of their solutions in a "good" way, since $88 \%$ agree to this statement. It seems that other geometry-related attributes, such as dimensions, size and appeal, cannot be ascertained to the same degree as pure geometry. Still, the quality is rated "good" by the majority of the

\footnotetext{
${ }^{1}$ Question: "How well can those product attributes, which are relevant for you, be identified with the help of a CAD model?"
} 
designers. Information regarding assembling attributes, kinematic behavior, manufacturing attributes and ergonomics are rated rather mediocre in average. Yet, only about $10 \%$ of the designers state that aspects of deformation and strength can be ascertained in "good" way with the help of CAD. In fact about $50 \%$ of the designers consider the possibility of ascertaining each of these attributes as "bad". This is very remarkable, since $62 \%$ of the designers claim that deformation and strength are important aspects of the design tasks they usually process.

The results presented above show that there is at least a potential lack of quality in the representation of certain product attributes with the help of CAD. Since sketches are used parallel to $\mathrm{CAD}$, it might be possible that sketches serve as an aid to represent those attributes. Therefore the designers were asked about which product attributes they usually represent with the help of sketches that are used parallel to CAD (see Figure 3-17).

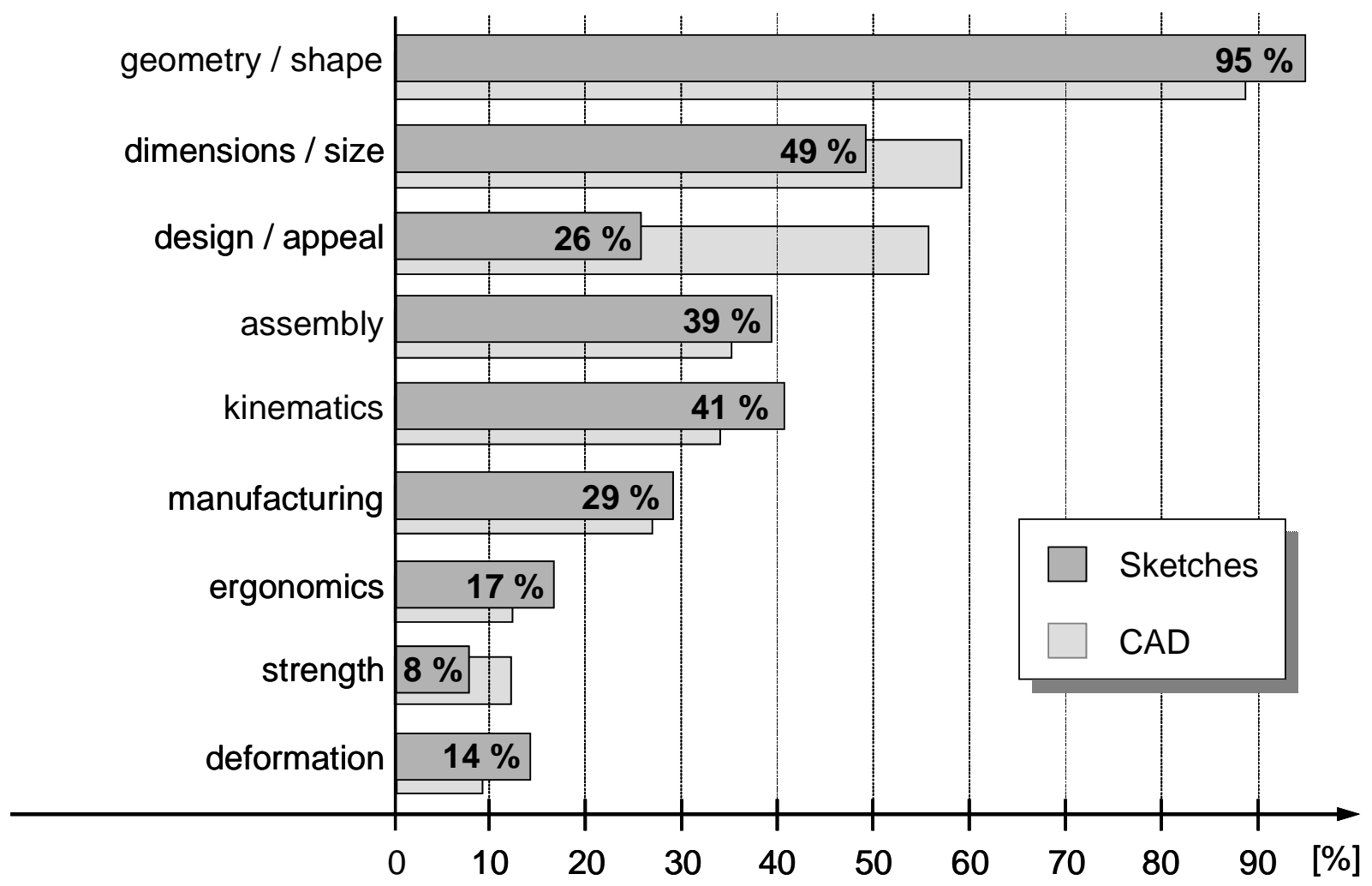

Figure 3-17: Product attributes represented by sketches ${ }^{1}$ (in comparison with those product attributes that are ascertained in a good way by CAD models - see Figure 3-16)

It shows that geometric information (95\%) dominate in sketches. Therefore it may be supposed that sketches, similar to CAD (as shown by light grey bars in Figure 3-17), represent this product property in a good or at least practical quality. Dimension and size

\footnotetext{
${ }^{1}$ Question: "What do you usually represent with the help of sketches that are used in parallel with CAD?"
} 
respectively are represented less often by sketches, probably due to the lack of accuracy in freehand sketches. Design, appeal or attractiveness is only represented by a quarter of the designers within sketches. For this purpose, CAD is probably the more practical tool due to its rendering functionality. Besides dimension/size and appeal, the profile of product attributes represented by sketches is rather similar to the profile of those attributes represented by CAD in a good way. So it does not seem as if sketches are used to represent those product attributes that are represented by CAD in a bad or impractical way. Sketches seem to supplement CAD in providing other functions (see Figure 3-15) but not in providing the representation of other product attributes.

A different picture emerges when the product attributes represented by Rapid Prototyping and Virtual Reality are considered (see Figure 3-18). However, these results should be used carefully, since only a rather small number of designers ever used Virtual Reality and/or Rapid Prototyping (Virtual Reality $17 \%$, Rapid Prototyping $52 \%$ ). The other designers were asked to answer the question about those tools hypothetically. Still, these results reflect the designers' expectations according to those tools. In the case of Rapid Prototyping, this especially applies to assembling properties and, in a minor extent, to manufacturing properties and ergonomics. In the case of Virtual Reality, this is noticeable for dimensions respectively size, assembling properties and ergonomics.

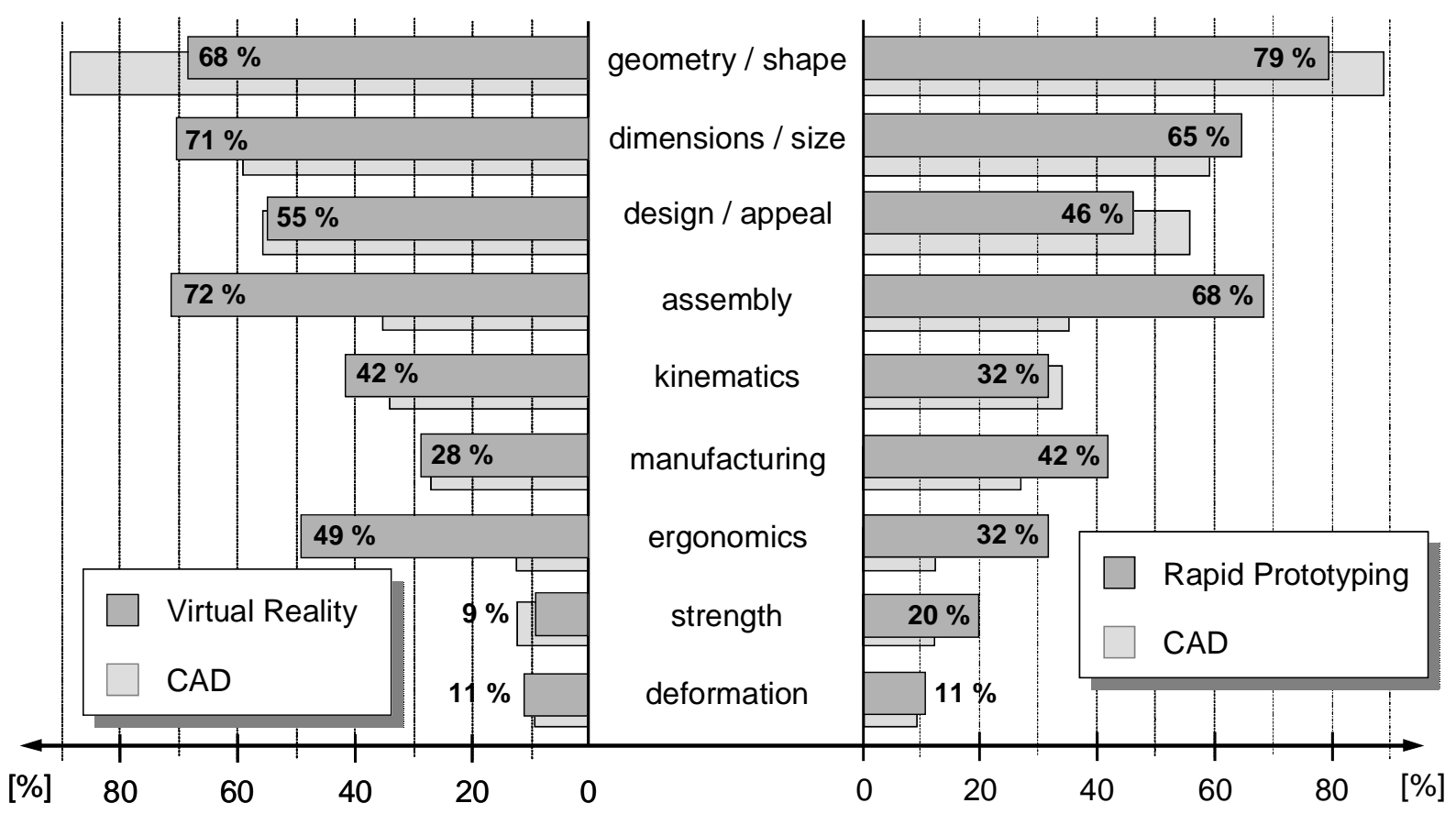

Figure 3-18: Product properties represented by Virtual Reality and Rapid Prototyping ${ }^{1}$ (in comparison with those product attributes that are ascertained in a good way by CAD models - see Figure 3-16) 
Although $20 \%$ of the designers (hypothetically) use Rapid Prototyping to represent aspects of strength, material behavior in general is represented rather seldom by Rapid Prototyping or Virtual Reality. Again, this is rather remarkable, since $62 \%$ of the designers claim that deformation and strength are aspects with great significance for the design tasks they usually process (see Figure 3-9). Asked for the characteristics of typical design tasks, this was even the item that was mentioned most often.

According to the results discussed above it seems that attributes of deformation and strength cannot be ascertained in a satisfying way with the help of CAD and sketches. Those media do not give an immediate feedback about these aspects of a product. Even rapid prototyping and virtual reality are used rather seldom to represent or analyze those aspects. It may be supposed that FEM serves the purpose to ascertain strength and deformation attributes since this is the main functionality of that tool. But this tool can only be used with a completed CAD-model and still requires further treatment such as the generation of a net. It remains uncertain, in which way designers determine a product's characteristics of deformation and strength. Designers probably use rough calculations, intuitive estimations and experience from previous design processes. Still, these processes are evidently not supported by common design media in a satisfying way. Due to the importance of aspects of deformation and strength in typical design tasks, developers of CAD should improve their systems by implementing such a functionality. But beforehand, design methodology should strive for the development of appropriate procedures and methods. There are scientific approaches to the integration of rough calculations in CAD (AMFT 2002), but they have not been implemented yet.

Besides strength and deformation, there is another indirect attribute that is regarded as being important in typical design tasks and is represented with the help of sketches rather often, which is kinematics. $41 \%$ of the designers state that they use sketches to represent kinematic behavior of products. Besides geometry/shape and dimensions/size (which are direct attributes), this is the attribute comes third and it is the indirect attribute that is named most often (see Figure 3-17). So the investigation of kinematics in sketches might be the best way to achieve an understanding on the representation of indirect attributes in general. From the way that kinematics are represented in sketches, it may be possible to infer suggestions on how to represent deformation and strength.

\subsubsection{Benefits from the Application of CAD and Sketches}

Designers seem to receive numerous positive benefits from the use of sketches and CAD. Considering the use of sketches, it seems that designers actually experience those advantages they want to achieve. The two benefits from the use of sketches that are named most often are improved communication (88 \%) and occurrence of new ideas $(77 \%)$. This corresponds to both the main intentions for the use of sketches parallel to $\mathrm{CAD}$, which are developing ideas

\footnotetext{
${ }^{1}$ Question: "Which attributes of your conceptual solution do you mostly wish to analyze or represent with the help of...?"
} 
and communication purposes. Furthermore, the simple comparison of solution alternatives (66\%) and the concretion of ideas with minor effort (53\%) are pointed out (see Figure 3-19).

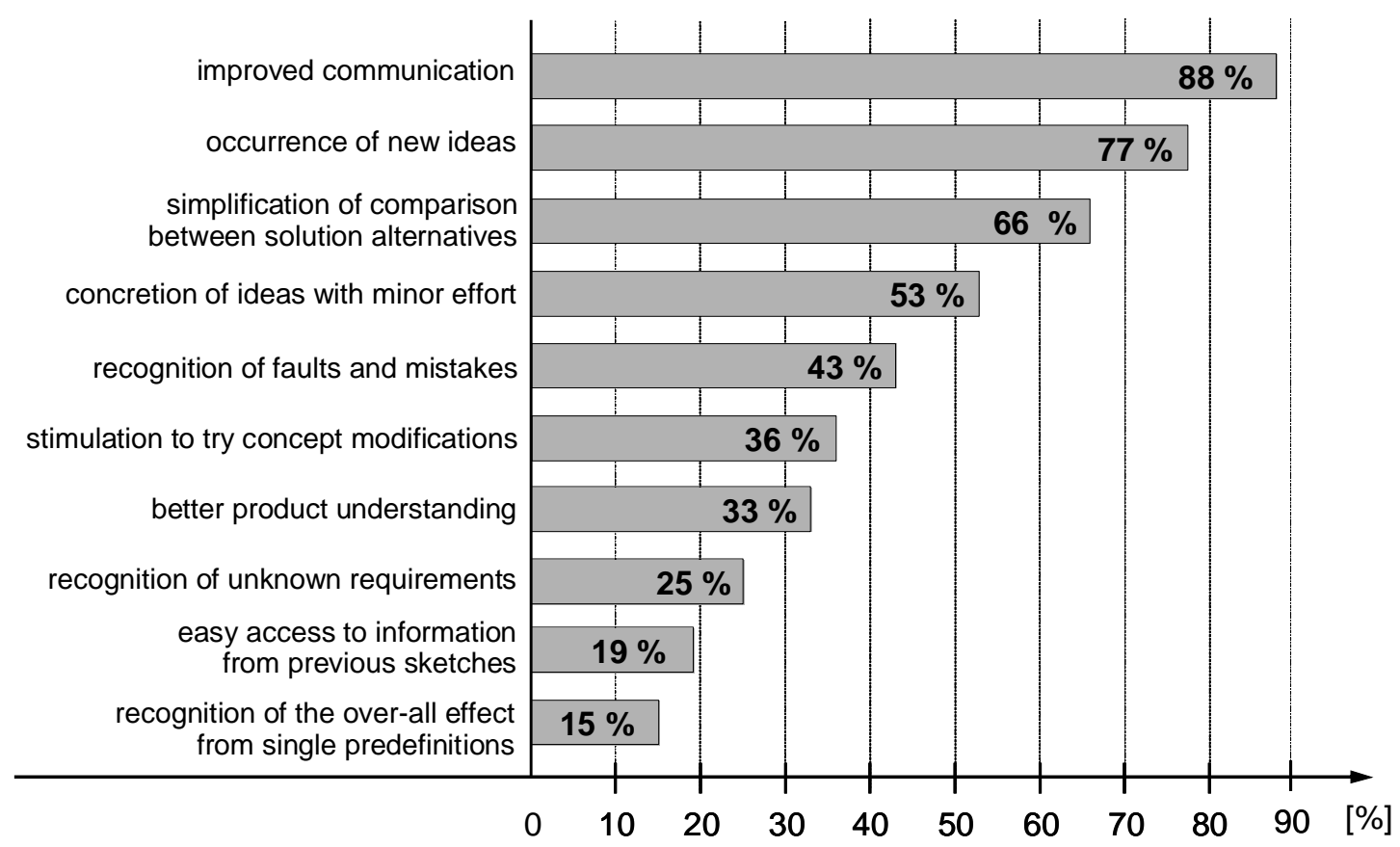

Figure 3-19: Effects from the use of sketches for conceptual design ${ }^{1}$

A different picture emerges when the benefits of utilizing CAD are considered. The recognition of faults and mistakes (75\%) is named most often as a common effect when CAD is used. Improved communication (72\%) and better understanding of the product are named often as well. The difference in the influence from CAD and sketches on the conceptual design process may be exemplified by the fact that occurrence of new ideas $(51 \%)$ only ranks sixth in the list of common benefits from the use of CAD (see Figure 3-20).

\footnotetext{
${ }^{1}$ Question: "Please choose a maximum of five statements that represent the support you received from sketches"
} 


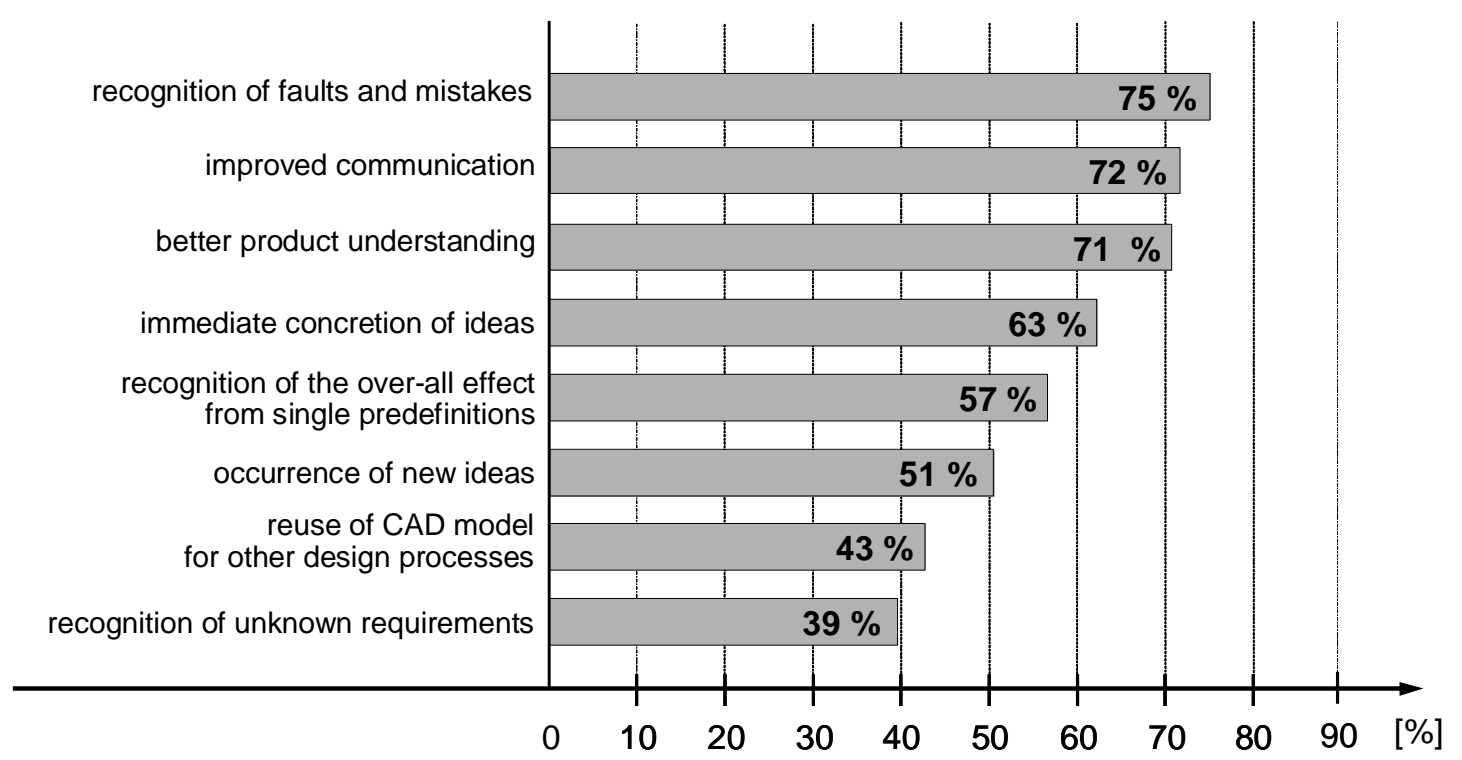

Figure 3-20: Effects from the use of CAD for conceptual design ${ }^{1}$

Regarding the overall impact from sketches and CAD on time and quality in conceptual design, the tools are subjectively judged in a different way by designers (see Figure 3-21). A large majority of designers $(85 \%)$ mainly agrees ${ }^{2}$ to the statement that better conceptual solutions arise when sketches are used. A different picture emerges for the designers' judgment concerning the impact of $\mathrm{CAD}$ on the quality of the conceptual solution. A narrow majority of the designers (54\%) again mainly agrees to the statement that better conceptual solutions arise when CAD is used, but there are $20 \%$ who mainly disagree. This result shows certain dissatisfaction with $\mathrm{CAD}$. It alludes either to the occurrence of mistakes when CAD is used or to obstacles on the way to the best solution when CAD is used.

\footnotetext{
${ }^{1}$ Question: "Which effects did you often perceive when you used CAD?"

2 In this context, "mainly agree" means that the statement is considered "absolutely right" or "mostly right". Likewise, "mainly disagree" means that the statement is considered "absolutely wrong" or "mostly wrong".
} 


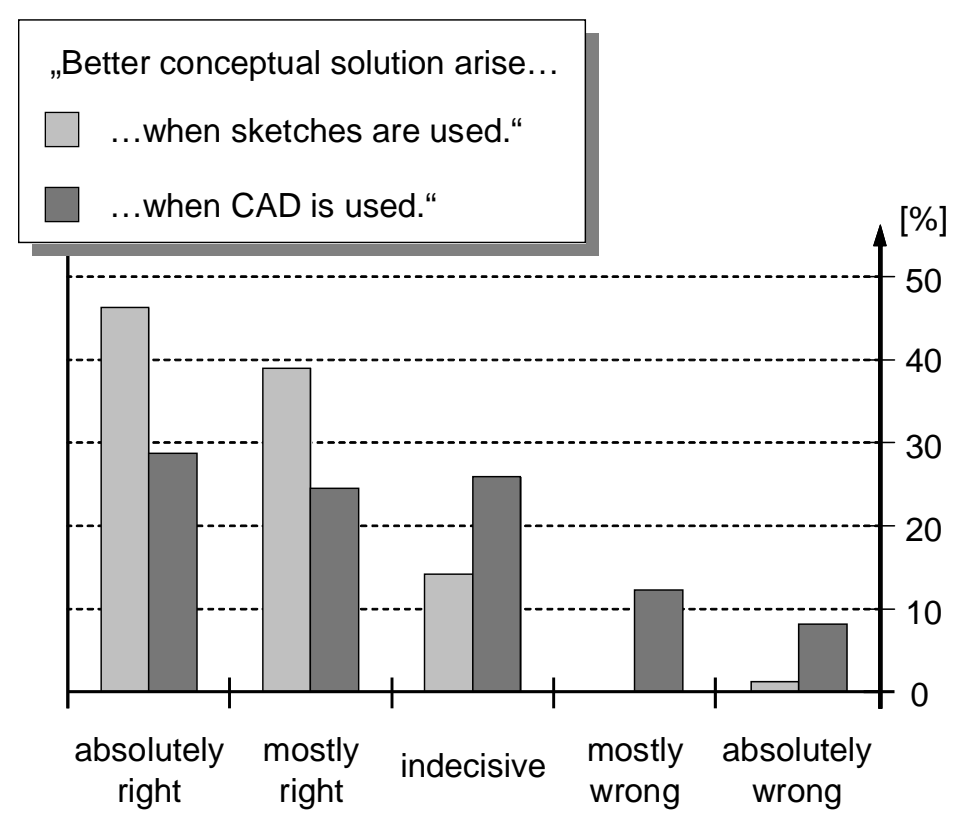

Figure 3-21: Designers' subjective judgment according to the general impact from sketches and CAD on the quality of conceptual solutions ${ }^{1}$

The results for the impact from sketches and CAD on the efficiency of conceptual design look rather similar (see Figure 3-22). Over $90 \%$ of the designers agree to the statement that the use of sketches leads to satisfactory conceptual solutions within a shorter amount of time than when no sketches are used. Although the majority of the designers (65\%) agree to the statement that satisfactory conceptual solutions arise faster when CAD is used, there are again $20 \%$ that disagree to this statement. This result may allude either again to the occurrence of time-consuming mistakes or to a general slowing down when CAD is used.

\footnotetext{
${ }^{1}$ Question: "Do you think that you develop better conceptual solutions whit ... than without ...?"
} 


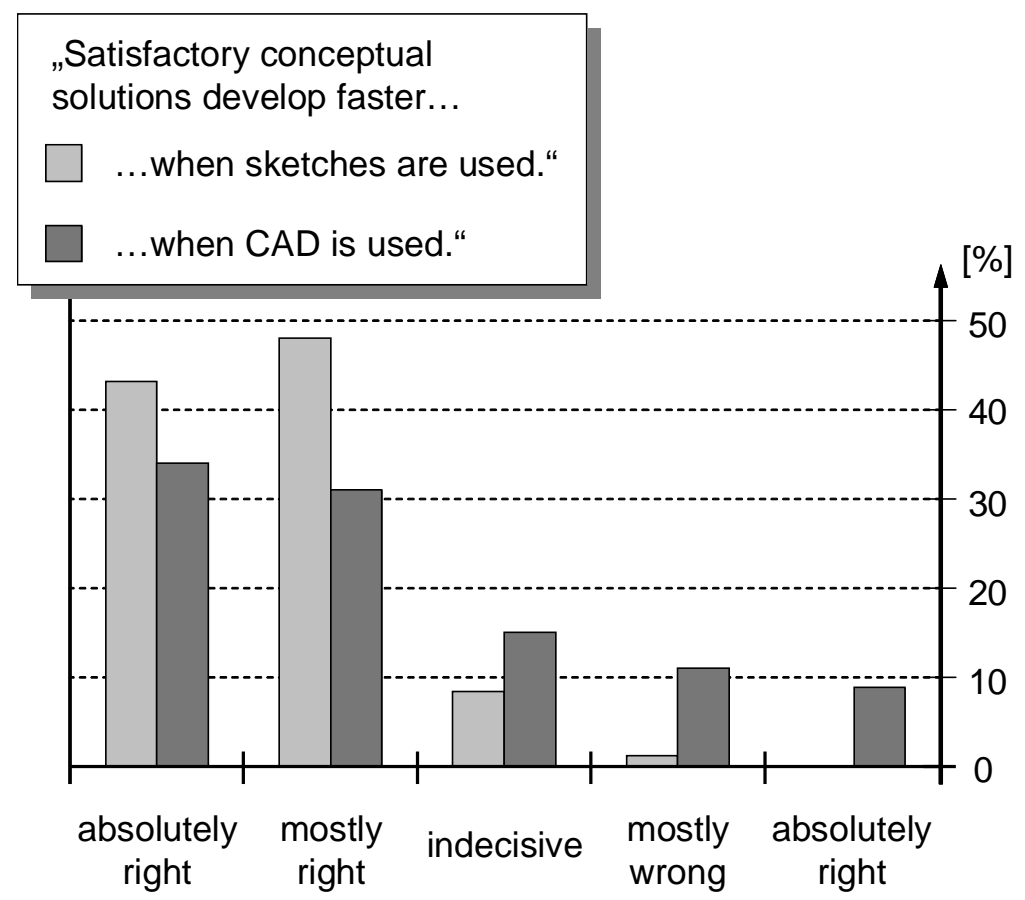

Figure 3-22: Designers' subjective judgment according to the general impact from sketches and CAD on the efficiency of conceptual design ${ }^{l}$

Further results provide clues to possible reasons for the less positive judgment of CAD by the designers. The survey also investigated more specific reasons for possible drawbacks from the use of CAD. The designers were asked for their subjective judgment according to the following questions:

- How intuitive is the operation of the CAD-system (see Figure 3-23)?

- How often does it occur that product predefinitions on CAD cannot be made in accordance with the own imagination (see Figure 3-24)?

- How high is the effort to change a CAD-model (see Figure 3-25)?

The results generally show a mediocre judgment. Still, $41 \%$ of the designers consider the intuitive character of their CAD-system's operation as low or even very low. Not one designer stated that the operation is very intuitive. And $19 \%$ of the designers state that predefinitions cannot be made in the desired way often or even very often. This is not the majority, but such an obstacle may prevent the designer in some cases from realizing the best solution concept although he may have developed it with the help of sketches already.

\footnotetext{
${ }^{1}$ Question: "Do you think that you develop a satisfactory conceptual solution faster with ... than without ...?"
} 


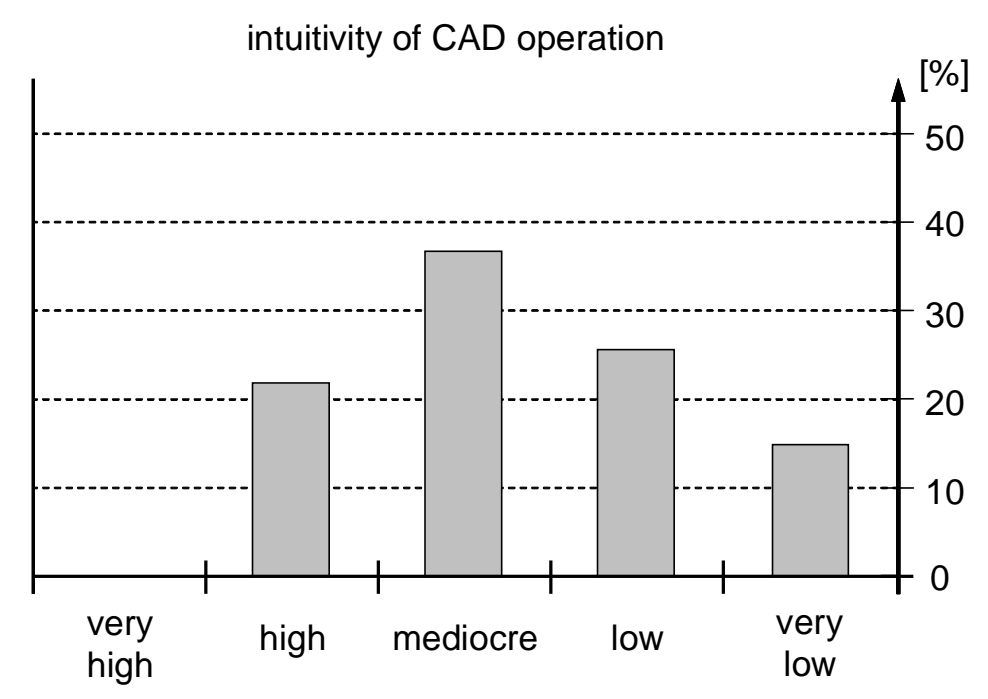

Figure 3-23: Designers' subjective judgment according to the intuitive character of CAD operation ${ }^{l}$

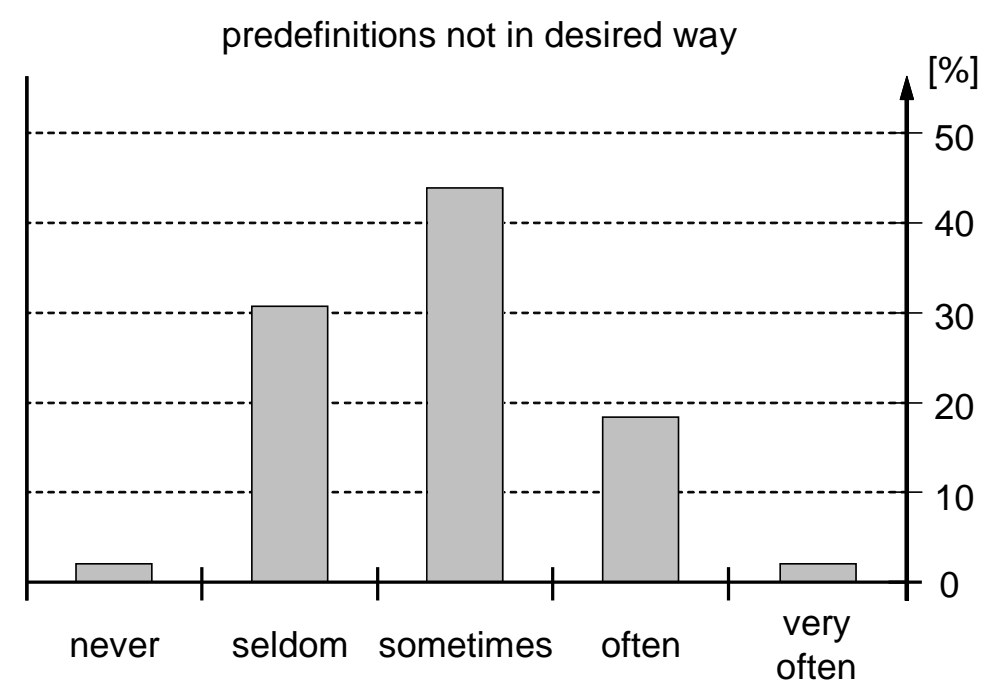

Figure 3-24: Frequency of situations, in which product predefinitions on CAD cannot be made in accordance with the imagination ${ }^{l}$

\footnotetext{
${ }^{1}$ Question: "How high do you consider the intuitive operability of the CAD-system?"
} 


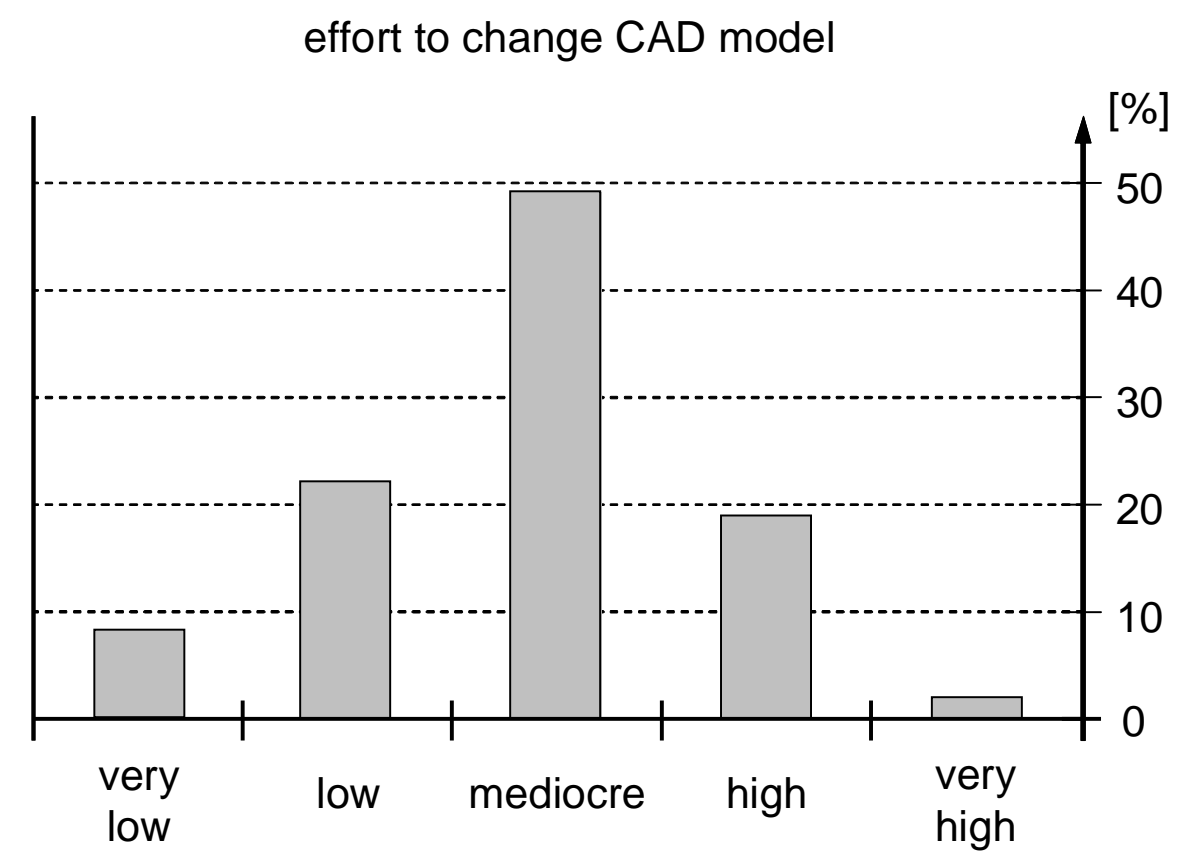

Figure 3-25: Designers' subjective judgment according to the effort for changes on a $C A D$ model $^{2}$

Although the results shown above only represent subjective judgments of designers (that were not gained by analyzing CAD itself), a dissatisfaction of designers becomes apparent that cannot be neglected. According to the European standard for software usability ISO 9241-1 (1997), usability of software is a product of effectiveness, efficiency and satisfaction of the user. Likewise, the dissatisfaction of the designers with their CAD-systems results in a lack of usability of the systems for conceptual design. One might argue that this lacking is constantly reduced e.g. by increasing speed of computation, growing memory capacity and by ergonomically (and aesthetically) improved user interfaces. At this point, we propose that this dissatisfaction reveals a general lack of usability of CAD-systems for conceptual design that results from the very core of the system. We propose that the CAD modeling methods and the ensuing design procedures are not suitable for conceptual design.

In the following considerations, we will outline a very basic view on design media and tools in general. This will provide us with an approach to examine creative mechanisms in conceptual design that occur on account of the medium or tool being used. We will use this

\footnotetext{
${ }^{1}$ Question: "How often can you not make predefinitions on CAD in accordance with your imagination?"

${ }^{2}$ Question: "How high do you consider the effort for changing CAD models?"
} 
approach to analyze the use of sketches and thereby elaborate why a sketch (as opposed to common CAD-systems) is a medium that is extremely suitable for conceptual design.

\subsection{Basic Characteristics of Media and Tools}

The characteristics of a medium determine in which way it can be used during design. Likewise, they determine the effect - positive or negative -on the result of the design process. We may consider a design medium just as storage for information, serving as a "backup" for the working memory. Yet, not only the storage of information within the medium needs to be considered, since the information needs to be entered into the medium before. Furthermore, if the medium is used as a temporary memory relief, the information has to be read out of the medium again. So the use of a design medium may include externalization of information and subsequent internalization of information. Both these processes comprise a number of complex sub-processes that depend on the user, the medium and the information. It needs to be regarded that the information undergoes certain alteration thereby.

Let us have a look at some examples of externalization: When a designer imagines a straight line and wants to draw it on a piece of paper, he needs to derive the motion of his hand from that imagination. This motion needs to have a certain direction, speed and duration in order to draw a line that represents the imagination. Such a derivation usually happens rather unconsciously but it may be necessary to carry out the process consciously once the imagination becomes more complex. In order to draw the imagination of a spatial object, it may require the conscious application of laws of perspective projection and drawing techniques. Yet, this process of derivation is completely different when a different medium is used. The advantage of 3D-CAD is that the application of such laws for depiction is not necessary. However, in that case a sequence of mouse clicks and entered values (according to the modeling mode of the system) needs to be derived from the imagination.

Especially in the case of $3 \mathrm{D}-\mathrm{CAD}$, it is obvious that internalization requires different subprocesses than externalization does. Inspecting and conceiving a spatial CAD-model demands the knowledge about the navigation mode of the system. Internalization should not just be regarded as the reverse-process of externalization. A solitarily working designer internalizes the same information he himself externalized before, but these processes are determined by the medium and may strongly differ for externalization and internalization. In the case of $\mathrm{CAD}$, it is imaginable that a person knows how to model geometric information, but does not know how to inspect the resulting CAD-model.

In the following chapter, we will consider the processes of externalization and internalization in theory and thereby develop a framework for subsequent empirical investigation of sketching in chapter 4 .

\subsubsection{A Model for Soliloquy using Media as Communication Channel}

For further consideration of the use of media, we will consult a model that explicitly allows to differ between externalization and internalization. Furthermore, it offers interesting clues for 
further reasoning that will be taken up later on. We will adapt the model of communication by SHANNON \& WEAVER (1949) to the use of design media. According to Shannon and Weaver, a general communication system consists of five elements:

- message source

- transmitter

- channel

- receiver

- message destination

Figure 3-26 shows the communication model schematically. The message source chooses a message for communication and hands it to the transmitter. The transmitter translates the message into a signal that is transferred to the receiver via a channel. The receiver retranslates the signal into a message and hands it over to the destination. Furthermore, the channel (and likewise the signal that passes the channel) may be subject to noise that is exerted on the channel.

Shannon \& Weaver $(1949$, p. 12) specify the problems that may occur during communication. They define three problem levels, which are characterized by the following questions:

- Level A:

How accurate is the signal transferred? (technical problem)

- Level B:

Do the transferred signals represent the initial meaning? (semantic problem)

- Level C:

How effectively does a message influence the receiver's behavior? (effectiveness problem)

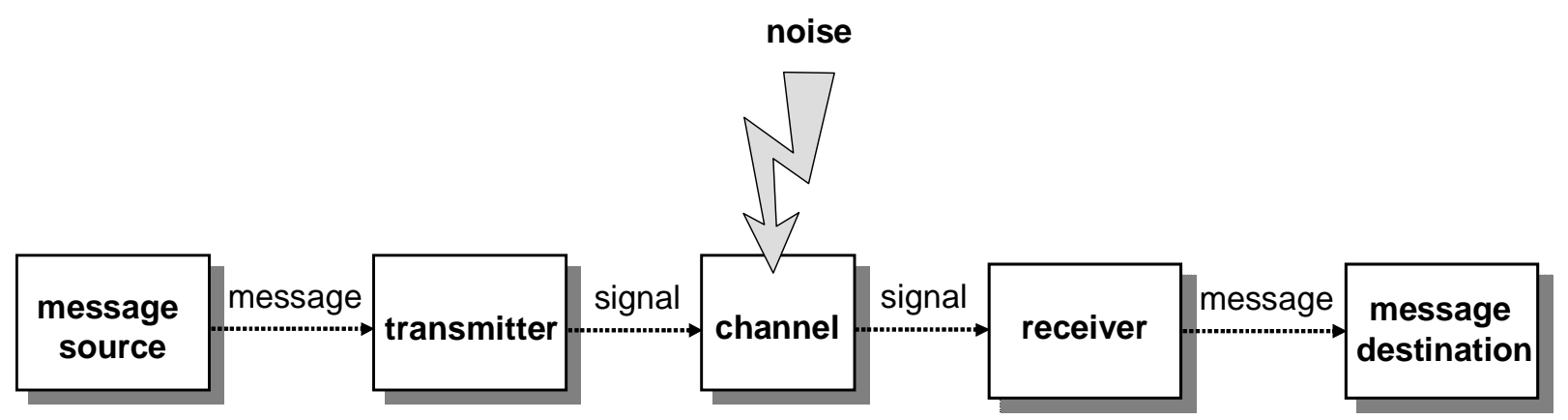

Figure 3-26: Communication system according to SHANNON \& WEAVER (1949)

Communication is defined as an exchange of information between individuals (MERRIAMWEBSTER 2003). So how could any form of communication take place when a designer 
sketches on his own while working solitarily on a design task? After all, there are not even two different individuals to exchange anything. Still, if the designer would collaborate with another, he could use a sketch for communication as well. According to surveys, $90 \%$ of the designers use sketches for communication to others, so a sketch is a feasible language for design communication. Still, another dominant intention for the use of sketches is the development of solutions, which is named likewise by more than $90 \%$ of the designers (PACHE et al. 1999).

There are results from studies that suggest that problem solving in general and design in particular may be seen as a process of communication with oneself. BARTL \& DÖRNER (1998) have shown through experiments that human beings tend to soliloquize while solving problems even when working on graphic problems. The manner in which this soliloquy is carried out effects the performance of problem solving. BERTAU (1999) showed that the more difficult the problem is, the more distinct this behavior becomes. The portion of expressions that show characteristics of a dialogue (questions, affirmations, etc.) increases with the difficulty of the problem. This even happens if there is no other person around. Bertau argues that the dialogue between the child and its mother is the first form of problem solving that is learned by a human being. Since more sophisticated methods for problem solving evolve from this process they still show characteristics of a dialogue as a relict from this origin. As a reminder: DÖRNER (1999) refers to problem solving as an "inner conversation of the soul with itself"!

SCHOEN (1983) describes professionals conducting dialogues with given situations as a source of creativity. Especially in the case of design, Schoen describes an architect having a reflective conversation with his own sketches, in which he externalizes his vague ideas and in turn finds useful clues for further refinement.

If Shannon and Beaver's communication model is applied to the soliloquy via sketches, then the information source and the destination would be the same - it would be the designer's mind. The resulting model is shown in Figure 3-27, in which the design media is the channel for communication.

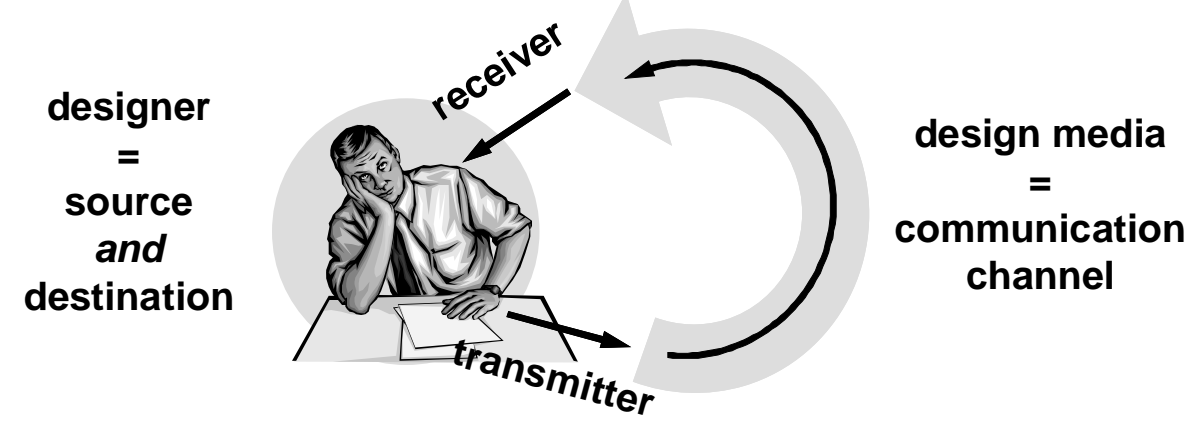

Figure 3-27: Application of the communication model according to SHANNON \& WEAVER (1949) on a designer sketching solitarily 
The "transmitter" contains all physical, psychical and technical processes of depicting information in a sketch. In the opposite manner, the "receiver" contains all processes of perceiving information from the sketch. Although information does not travel over long distances via sketches, some kind of "noise" may occur that is exerted upon this communication channel. Thereby the information may be altered during the soliloquy without the ability of the designer to prevent or affect this alteration.

A simple example can be used to show why "noise" may occur in the case of sketching. Figure 3-28 (a) shows two straight lines that run parallel. If one would have drawn these lines with the help of a ruler and then added the bundle of rays shown in Figure 3-28 (b), the result would be that the lines are not perceived as being parallel anymore (see Figure 3-28 c) This optical Illusion is known as the Hering effect ${ }^{1}$. One may still remember that the lines are parallel, but then the depiction of the lines is no memory relief because he or she had to keep that in mind. So the noise that we have discovered in this case results from the interference between different pieces of information that are received from the channel at the same time.

a)

b)
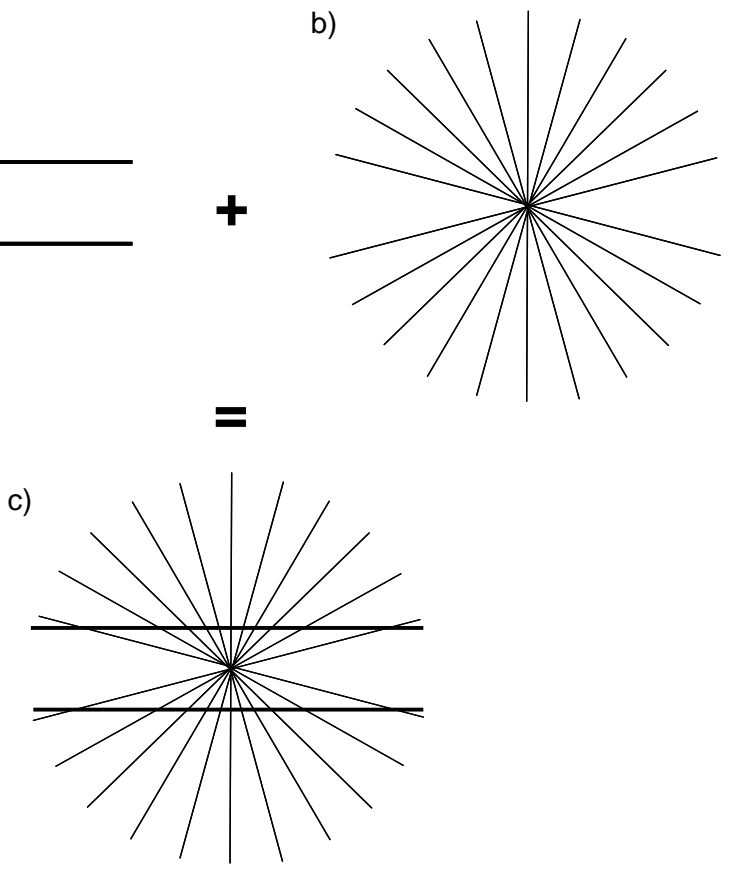

Figure 3-28: Geometrical-optical illusion resulting from the interference between different pieces of visual information

We will consider the application of this communication model on sketching media in greater detail later on. At first, we will have a differential look at tools for design.

\footnotetext{
${ }^{1}$ This effect was discovered by Ewald Hering in 1861 (see BACH 2003 for a digital animation of numerous effects of optical illusions).
} 


\subsubsection{A Differentiation of Tools for Design}

At the end of the preceding chapter, a simple tool has been mentioned and that has been a ruler. It allows the designer to draw a straight line that is more precise than a free-hand line, so the ruler intensifies the designer's accuracy in drawing. In the following considerations, we will regard any object or device that intensifies the human action as an instrument (HACKER 1998, pp.159). A hammer is a classic example for an instrument - it intensifies human strength by the effect of inertia ${ }^{1}$. We will use this definition also in a more broad sense for devices that intensify not only mechanical, but also mental action.

Notice: In the context of sketching, one might regard the pen as an instrument as well. Without the pen, the sketch would be no medium at all so the pen is considered as being a part of the medium sketch. Therefore, the pen will not be regarded as an instrument.

In contradiction to an instrument, an automatic device substitutes human action (HACKER 1998, pp.159). Thereby, such a device undertakes to regulate the action and this is actually the essence of automation. We will call such a device an automat and a functionality that substitutes human action as an automatism. CAD acts at least partially as an automat, since it provides the designer with many automatisms. The system may take over the calculation of volume, mass and moment of inertia for the designer or it may check an assembly for collisions.

Still, the differentiation between an instrument and an automat does not categorically depend on the device. The example of drawing a line can be used to show that this differentiation rather depends on the action that is considered. If the designer simply wants to produce a straight line than both the ruler and the CAD-system may be regarded as an instrument. It does not matter whether the regulation of the motion's direction is made unnecessary by the ruler or the motion itself is substituted by the CAD-system. They both simply intensify the accuracy of the line in comparison to not having one of those devices.

If the process of drawing a line of any shape is considered - maybe a line that represents the curve of the nose in a portrait - then the ruler is quite useless, since it inhibits drawing a curved line. A CAD-system may be used for that purpose, but the production of that line is the application of an automatism. The designer specifies several points that the line shall pass through and he gives some information about the run of the line between those hold points. He sets the goal for the process, but the system carries out the execution of the process. The designer is not able to vary the run of the line simultaneously while it is being created.

The previous example of drawing a line that is part of a portrait shows a basic problem of automation. The author supposes that portrait painters agree that it is hardly possible to draw a portrait with the help of vector-based graphics software or even a CAD-system. Drawing such a line is a continuous process that requires continuous regulation. At every moment of the

\footnotetext{
${ }^{1}$ The term tool would better correspond to the colloquial understanding of such device as a hammer. But in the technical area, the term tool is so universally and yet incorrectly used, so we will also use it as a term for any support device.
} 
process, the actual object that is portrayed and the part of the line that has been drawn so far determine the continuation of the line. Portraying is different from taking a photo. The visual extraction of a characteristic line out of the complex sight of a human face is a complex process of perception. The author has to admit that he himself cannot separate the visual extraction and isolation of the line from the process of drawing. For him, the line disengages from the sight while he sketches, so he would not be able to imagine the run of the line in advance. The reader is invited to carry out this experiment on oneself (the author would appreciate any comment on this subject).

In the following chapter, we will investigate real sketching processes that have been carried out during conceptual design. The free-hand line, which is explicitly drawn without the help of a tool that intensifies its accuracy, will play an essential role then. The soliloquy model that has been introduced just before will help us to integrate case-based observations on sketching into the greater context of creativity and conceptual design. These observations will lay ground for the conception of the new sketching media that will be presented in chapter 5 . At that point, the question will reemerge whether or not such a medium should provide intensification or even automation of human action. 
This book is available at Verlag Dr. Hut, München, www.dr.hut-verlag.de (ISBN 3-89963-176-5) 


\section{Sketching - A Major Element of Conceptual Design}

The results from both the surveys presented in chapter 3 showed the dominance of sketches and $\mathrm{CAD}$ as major representational media for conceptual design. It seems that sketches support the process of idea development for conceptual solutions to a greater extent than CAD. Furthermore, it seems that sketches are used to supplement CAD concerning this function when used parallel to CAD. Sketches serve this purpose because the occurrence of new ideas is stated as being the second most important benefit of sketches besides improved communication. In order to examine the creative mechanisms initiated and supported by sketches, an extensive experimental study was carried out to examine the use of sketches.

Notice: In the following chapters, numerous scans of design sketches will be presented. The scanning process was set up in such a way that all elements of the sketch - even very slight lines - are visible. Therefore, some sketches appear to be darker and richer in contrast than in reality. Nevertheless, this seemingly poor quality of the scan serves the purpose of better understanding the content of the sketch.

\subsection{An Experimental Investigation on Design Sketching}

The sample of test subjects for the experimental investigation consisted of 61 persons overall. It contained 46 students of an engineering course of studies, of which $78 \%$ studied mechanical engineering. $20 \%$ studied electrical engineering but gained knowledge in mechanical design through practical training or lectures. One student $(2 \%)$ studied process engineering. On average, the students were at the age of 23.5 years. Furthermore, 15 designers from industry were investigated. About half of them worked in branches that could be assigned to mechanical engineering ( $47 \%$ ), the others worked in the field of automotive engineering $(53 \%)$. The majority of the designers was educated at a German "Fachhochschule" $(67 \%)$ and $20 \%$ studied at a technical University ${ }^{2}$. The others were educated as draftsmen for example. The majority (73\%) stated that they mainly work on original design tasks while the others mostly carry out adaptive design. On average, the designers were 35 years old and had 9 years of working experience on average.

For the experimental investigation each test subject had to work on two design tasks in order to find a conceptual solution. The design tasks were worked out in a way that they hypothetically make different demands on the design ability of the test subjects ${ }^{3}$. The less

\footnotetext{
${ }^{1}$ The diploma from a „Fachhochschule“ is more or less comparable to a bachelor degree.

${ }^{2}$ Likewise, the diploma from a university is comparable to a master degree.

${ }^{3}$ The design tasks showed different values according to the method for analysis of the demand structure by SCHRODA (2000). This method determines the degree of demand as a combination of complexity, intransparency, degrees of freedom, dynamism, required knowledge and the existence of contradictory goals.
} 
difficult design task ${ }^{1}$ concerns the design of an adjustable barbecue grill that is infinitely variable according to the distance between the coals and the grilled meat. Thereby no manual contact to the grate and the coal tub should be necessary for adjustment (even not with a pair of pliers or any similar removable handle). Figure 4-1 exemplifies these circumstances schematically. Additionally, both the grate and the tub should be easily removed for cleaning. All components near the coal tub should be heatproof. Furthermore, weather resistance was demanded and the use of electrical components was prohibited. The conceptual solution for the grill was supposed to be achieved within approximately one hour.

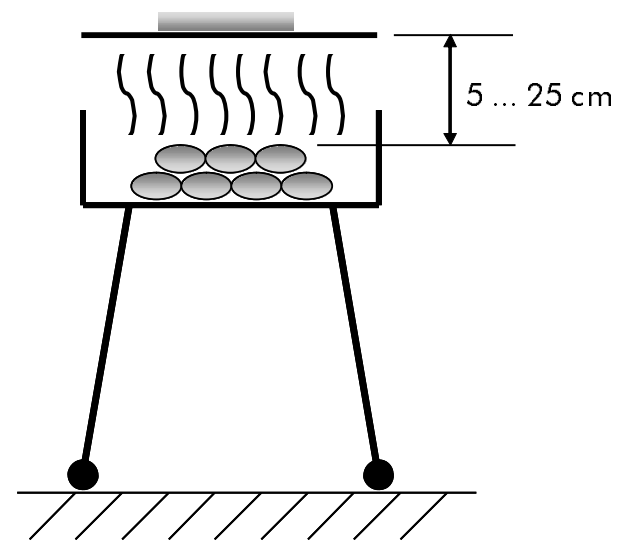

Figure 4-1: Exemplification of the design task "barbecue grill"

In the second, more difficult design $\operatorname{task}^{2}$, the test subjects were asked to design the conceptual solution for a device for laser welding of car body sheets. As boundary conditions, the laser and a linear motor for actuation of the laser were given, as well as shape and dimension of the sheet metal (see Figure 4-2). Each test subject had to develop a device that made the laser move with a defined speed and a defined distance along the sheet metal. The laser beam had to hit the metal's surface at right angles all the way. For cost reasons, the linear drive was the only external actuation that was allowed to be used for motion of the laser (Still, the test subject could try to use e. g. gravity for actuation).

\footnotetext{
${ }^{1}$ According to the method from SCHRODA (2000), this design task can be regarded as "simple adaptive design".

${ }^{2}$ According to the method from SCHRODA (2000), this design task can be regarded as "complex adaptive design".
} 


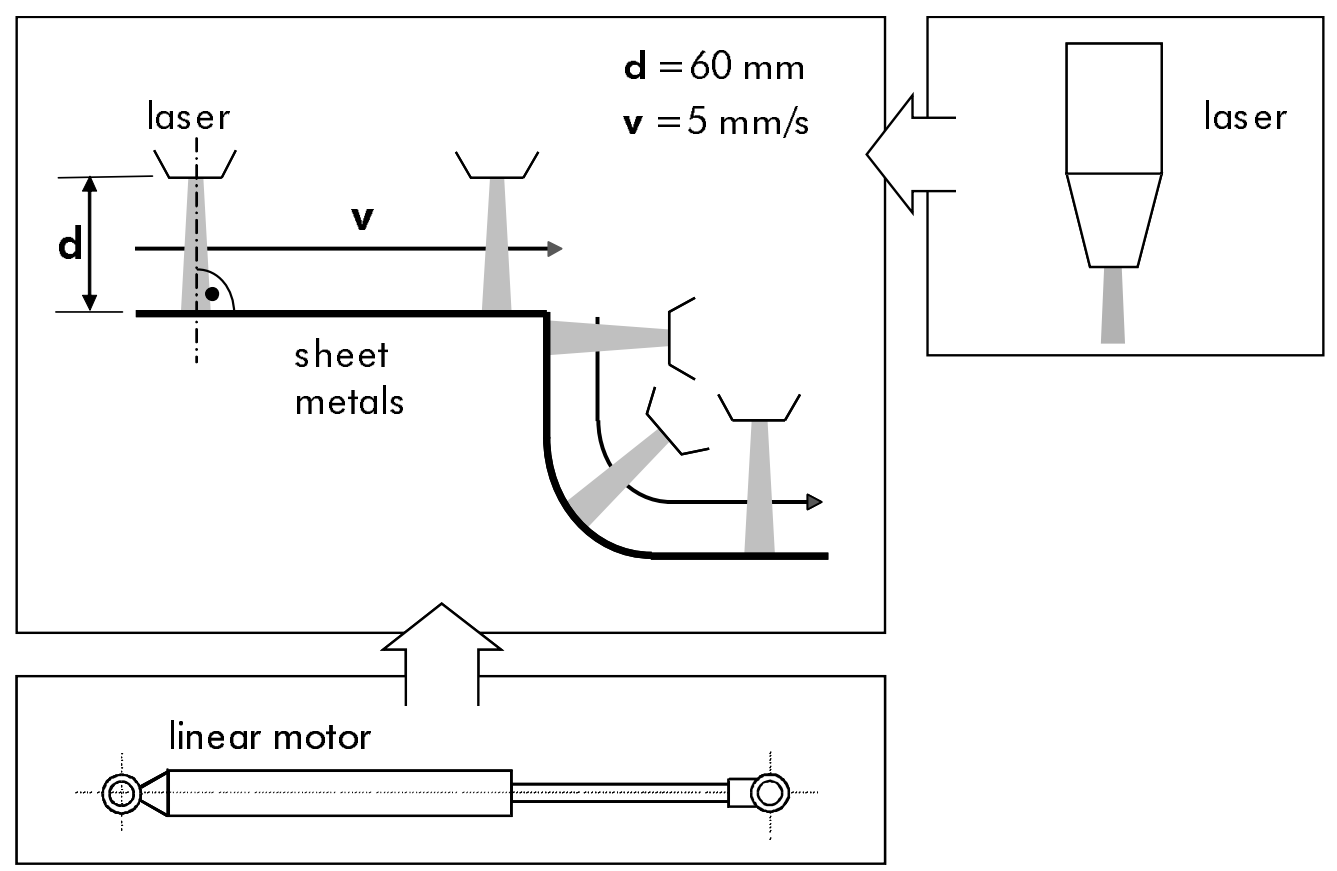

Figure 4-2: The design task "laser welding device"

The graphical information that was given to the test subject in addition to the verbal description is shown in Figure 4-3.

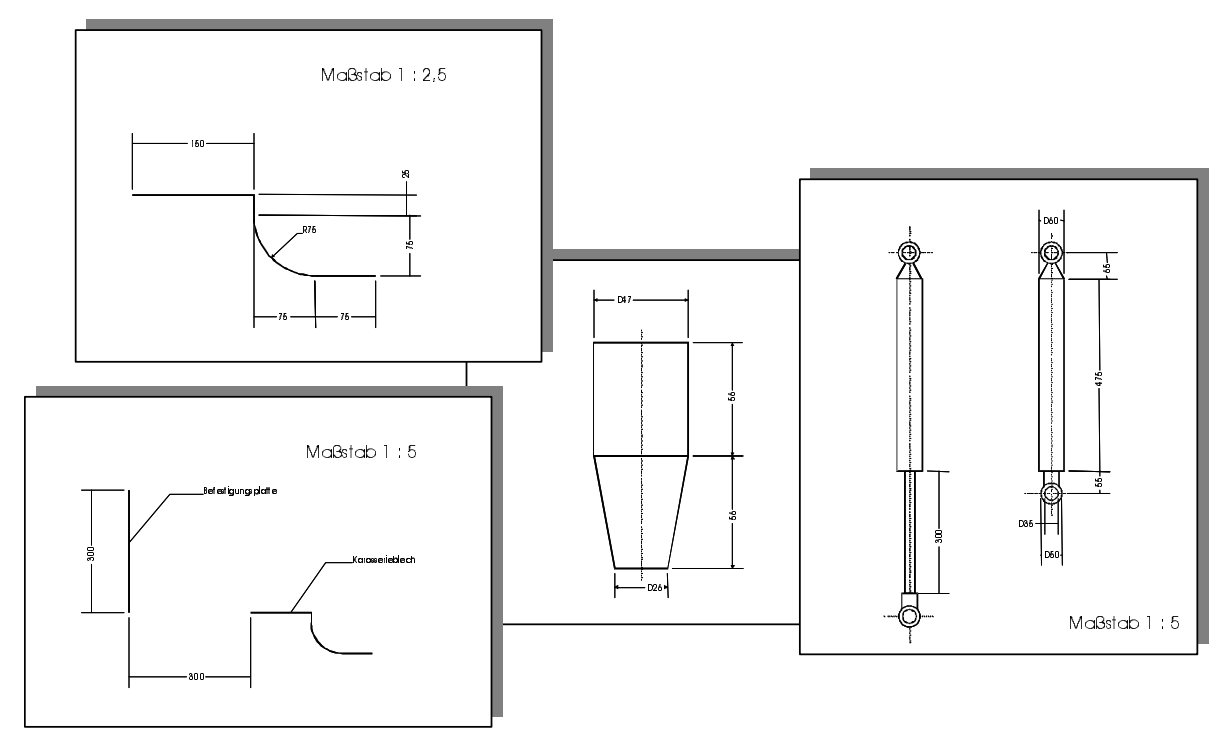

Figure 4-3: Graphical information given in the design task "laser welding device" 
The reader is invited to try to solve this problem himself. The complete design task is given in the appendix. We will address some of the major difficulties of the design task, which were frequently sources of error. Furthermore, some of the standard solution approaches developed by the test subjects will be presented. So those readers that want to challenge themselves by working on the design task without any hint at solution possibilities, should go on reading on page 76 .

One major difficulty of the laser welding task consists of the fact that - as evident in Figure 4-4 a) - the sheet metal consists of straight edges as well as of curved edges. Therefore, the linear motion from the drive has to be transformed into a nonlinear motion of the laser. There is also a right-angled corner that needs to be passed (see Figure 4-4 b). At this point, the laser needs to be switched off for the time it is put from the end of the previous straight edge to the following edge. Otherwise the corner might get burned during that time. Gravity cannot be used directly to actuate the laser (e. g. at the vertical segment of the sheet, see Figure 4-4 c) because than the laser will accelerate although it is supposed to move with a constant speed. Additionally, the distance covered by the motion of the laser is longer than the motion range of the linear drive (see Figure 4-4 d) and thus, a solution that overcomes this discrepancy needs to be found. The laser needs to be returned to the starting point after the welding process in order to weld the next sheet (see Figure 4-4 e). The latter requirement is not given explicitly in the task, although this information may be gathered indirectly from the task description. Furthermore, some common requirements (e. g. maximum weight and dimension) are stated.

a) transmission of a linear motion into a non-linear motion
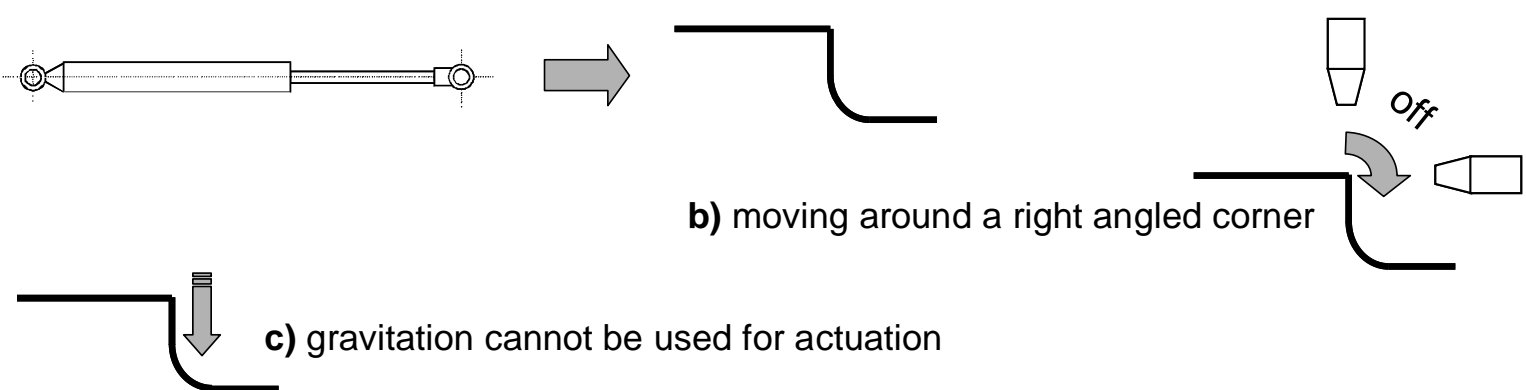

d) length of the laser's way is longer than the actuation way of the linear motor

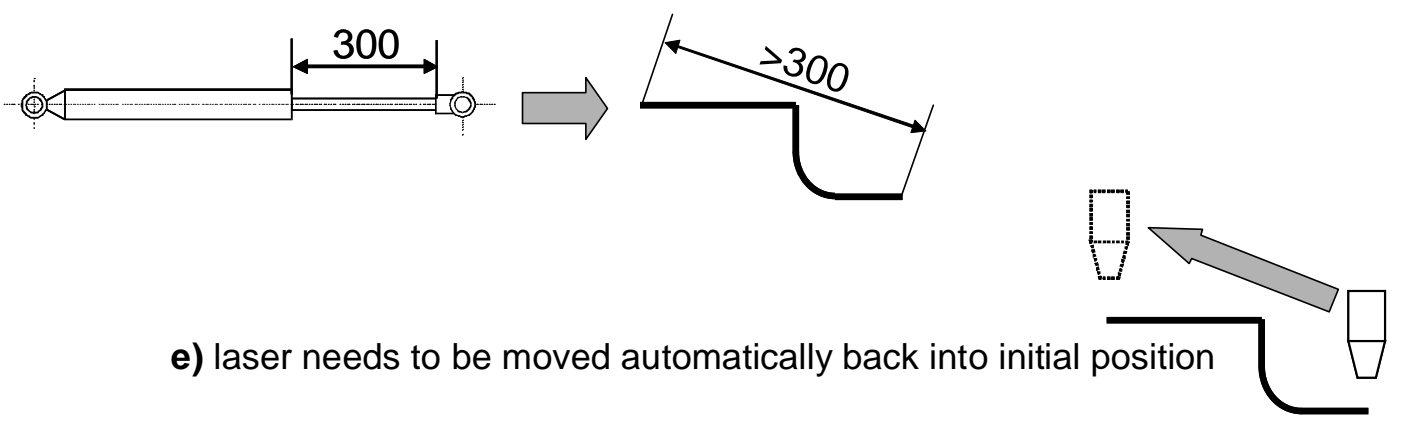

Figure 4-4: Specific difficulties in designing the laser welding device

This book is available at Verlag Dr. Hut, München, www.dr.hut-verlag.de (ISBN 3-89963-176-5) 
One solution approach that has proven to be rather successful in some of the experiments is shown in Figure 4-5. The desired direction of the laser's motion is achieved by a track that is shaped in the way the laser is supposed to move. The laser is connected to a "crab" that runs in these tracks. Thereby the laser perforce has to move along the shape of the tracks. The crab has two wheels so it always runs parallel to the tangent of the track's shape. The laser is fixed to the crab at a right angle, so the laser beam hits the sheet metal (that is located underneath the track) at a right-angle as well. Still, the portion that is covered by the laser is longer than the motion range of the linear drive. This problem can be solved by attaching the drive to any kind of fixture above the track with the help of a joint. During actuation, the laser rotates around the joint. In the upper right corner of Figure 4-5, the principle of transformation is schematically illustrated. When the linear drive extends by a certain length $\left(d_{\text {actuation }}\right)$, it accordingly rotates around the joint for a certain angle $(\alpha)$. The resulting length of the laser's motion $\left(\mathrm{d}_{\text {laser }}\right)$ is longer than the extension of the linear drive.

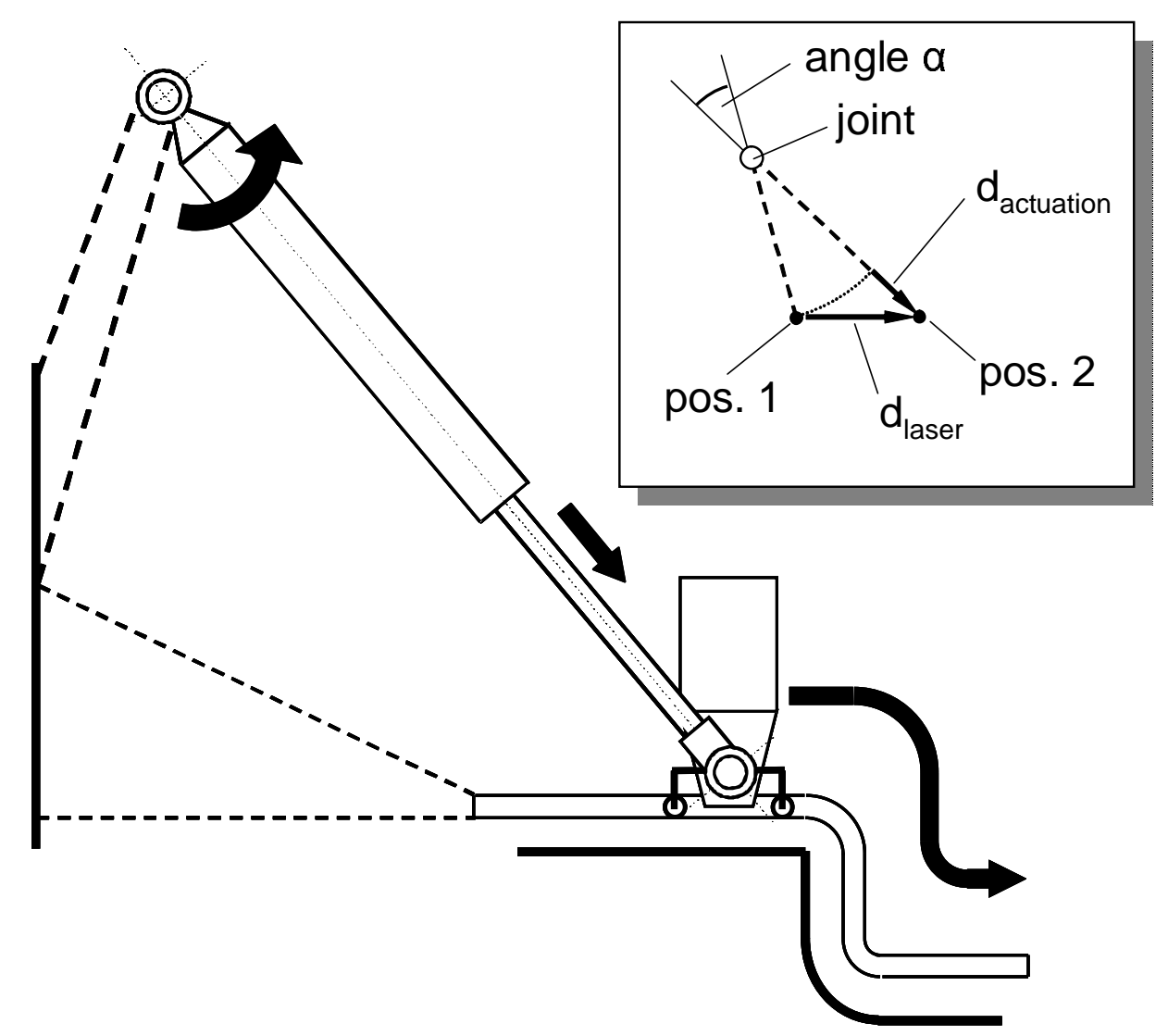

Figure 4-5: Solution approach fort he laser welding task using a crab that runs in a track

Another useful solution approach uses a belt to guide the laser (see Figure 4-6). The belt is circulating around several coils. Since the desired motion of the laser consists of straight sections and circle segments, the belt can be forced into a shape that matches the desired 
laser's motion. The laser is attached at a right angle to the belt and as soon as the belt circulates, the laser moves along the sheet metal. In order to overcome the discrepancy between the distance covered by the laser's motion and the range of the linear drive, the belt can be actuated by a wheel. This wheel is actuated by the linear drive by means of a crank mechanism, similar to the actuation of the wheels of a steam train.

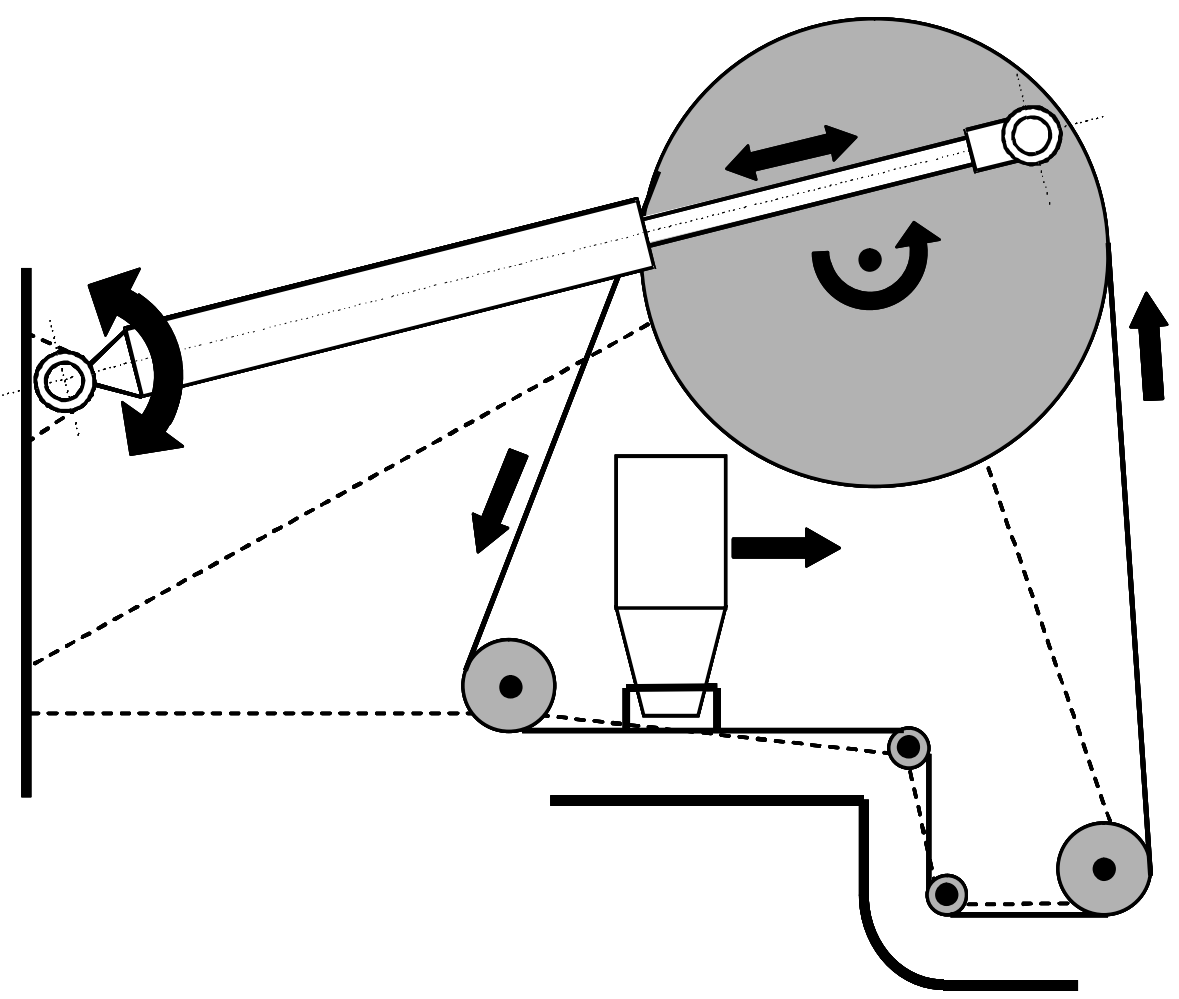

Figure 4-6: Solution approach for the laser welding task using a belt that is actuated by a crank mechanism

The experiments were designed to clarify a number of questions concerning the use of sketches for design. First of all, it should be clarified, if sketches are really used to such an extent that was indicated by the surveys. Therefore, the sample of test subjects was divided in two different experimental groups: one group that was requested to use sketches (and models ${ }^{1}$ ) and another group without such a request for sketching (and modeling). In the group with no request for sketching or modeling, no material was presented. If required, material was provided (e.g. paper, pencils, pasteboard, wire, a model kit, plasticine etc.). The test subjects requested to sketch or model were explicitly invited to use the materials that were made available. The groups consisted of 30 and 31 persons respectively and the persons were randomly assigned to each group. The experimental design is shown in Table 4-1.

\footnotetext{
${ }^{1}$ By these experiments, the use of simple physical models was investigated as well. Still, in the following this issue is not elaborated further. For results on the use of models, see RöMER (2002).
} 
Table 4-1: Experimental Design

\begin{tabular}{|c|c|c|c|}
\hline & \multicolumn{2}{|c|}{ design task } \\
\hline & & barbecue grill & laser welding \\
\hline \multirow{2}{*}{$\begin{array}{r}\text { request for } \\
\text { sketching }\end{array}$} & with & \multicolumn{2}{|c|}{23 students and 7 designers } \\
\hline & without & \multicolumn{2}{|c|}{23 students and 8 designers } \\
\hline
\end{tabular}

The design process was recorded by audio and video equipment and the test subject's actions were on-line-documented by means of a computer-based protocol. The protocol differentiated between the following categories of action (among others):

- writing,

- reading,

- sketching,

- erasing,

- regarding (the sketch),

- talking,

- gesturing and

- "staring into space ${ }^{1 "}$.

Personal data were gathered, i.e. experience in design, design education, ability for spatial imagination (GITTLER 1990) and heuristic competence (STÄUDEL 1988). After the experiment, the test subjects were interviewed about the run of the design process and the functional principle of the solution they developed. The video recording allowed additional case study of solution progress, problem solving steps and the solution generated.

\footnotetext{
${ }^{1}$ This category includes those actions that could not unequivocally be assigned any one of the other categories.
} 


\subsection{The General Characteristics of a Sketching Process in Conceptual Design}

First of all, the experiments show that the use of sketches de facto is a common media for conceptual design, especially in the case of difficult and demanding design tasks. All test subjects used sketches to work on the laser welding task and only three persons from the group that was not been requested to sketch did not sketch (see Table 4-2). In fact, it could be argued that there was no solution development by way of sketches in these three cases; the test subjects stated that they developed an entire conceptual solution in their head and then sketched the solution afterwards in order to document it. Due to the experimental setting, it could not be verified if the solution was either developed further or modified during that documentation.

Table 4-2: Numbers of test subjects that used sketches

\begin{tabular}{|r|l|l|}
\cline { 2 - 3 } \multicolumn{1}{c|}{} & \multicolumn{2}{c|}{ design task } \\
\hline \multirow{2}{*}{$\begin{array}{r}\text { request for } \\
\text { sketching with }\end{array}$} & $\begin{array}{l}30 \text { persons } \\
\text { used sketches }\end{array}$ & $\begin{array}{l}\text { 30 persons } \\
\text { used sketches }\end{array}$ \\
\cline { 2 - 3 } without & $\begin{array}{l}28 \text { persons } \\
\text { used sketches, } \\
\text { 3 persons } \\
\text { used no media }\end{array}$ & $\begin{array}{l}31 \text { persons } \\
\text { used sketches }\end{array}$ \\
\hline
\end{tabular}

Through analysis of the test subject's actions, it has shown that the rates of sketching activity in relation to the overall process strongly differ for different test subjects. For sake of clarity, only the results for the professional designers are charted in Figure 4-7. For the designers, the average rate of sketching activity (referring to both design tasks) ranges from about $17 \%$ to $44 \%$. The results for the students are similar (in that case, the lowest average value is about $14 \%$ and the highest value is $42 \%$ ). So it seems that there is not one common procedure of conceptual solution development according to the intensity of sketching activity. Moreover, no correlation could be found between the rates of sketching activity and the kind of task according to each person. Thus, there seems to be no individual style of sketching intensity that is independent from the task. 


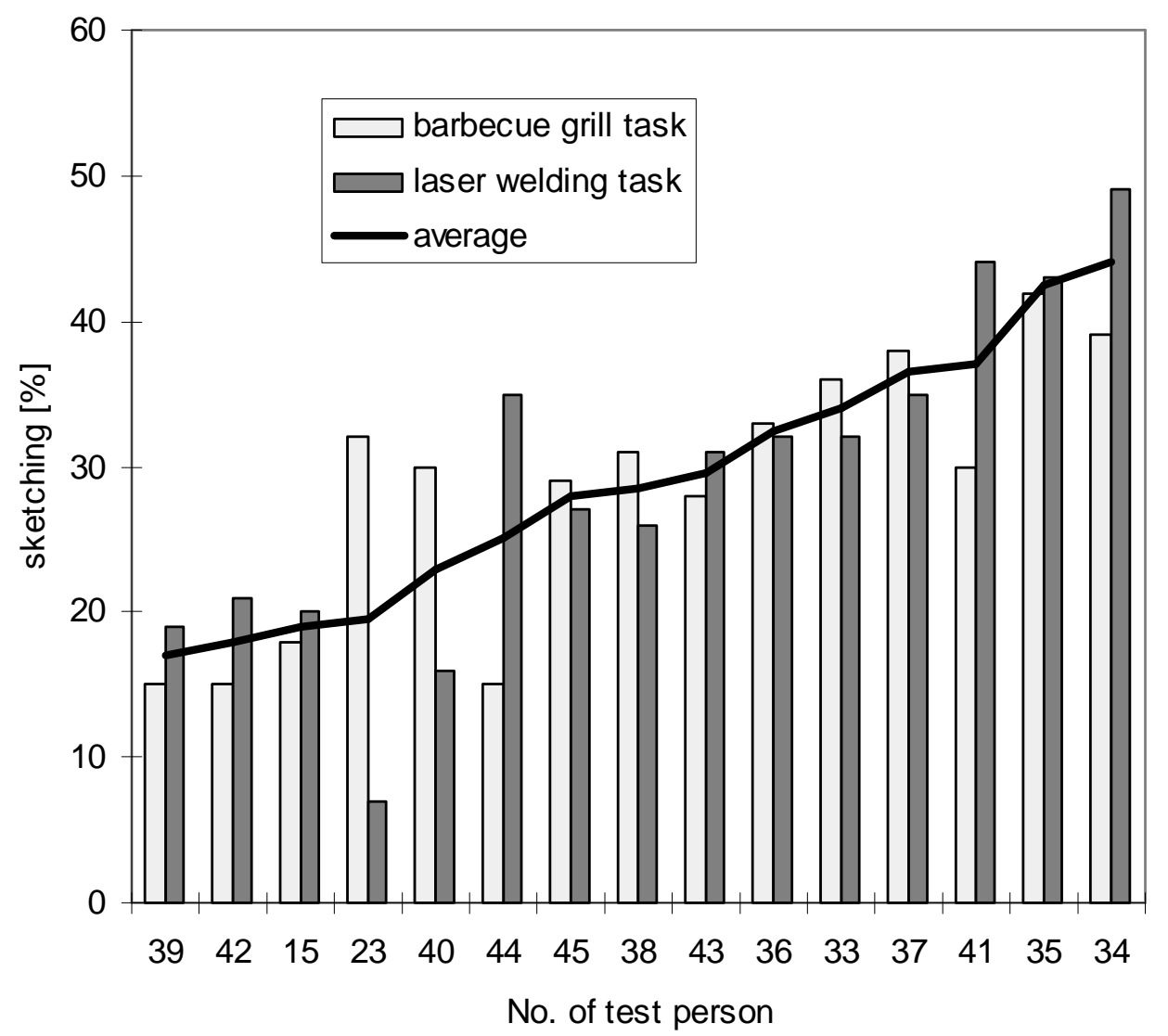

Figure 4-7: Designers' rate of sketching action referred to the overall time

Regarding the results shown in Figure 4-7, the test subjects no. 39 (average sketching activity $17 \%$ ) and no. 34 (average sketching activity $44 \%$ ) appear to be corner cases among the designers according to their sketching intensity. Considering the distribution of all actions over the whole duration of work on the laser welding task, it shows that the activity profiles for these corner cases strongly differ (see Figure 4-8). Such a basic activity as erasing does not even occur at all in the case of no. 34. This person has not used an eraser throughout the whole process of solution development although it was available - this points out, that there may be individual sketching styles that differ fundamentally for different persons. Still, both test subjects work with the sketch for about $75 \%$ of the time ${ }^{1}$. Therefore it should not be supposed that test subject no. 39 did benefit less from his sketches during the solution development process, just because he sketched only for $17 \%$ of the overall time.

\footnotetext{
${ }^{1}$ In that case, the actions sketching, erasing and watching the sketch are summarized to the action work with the sketch.
} 


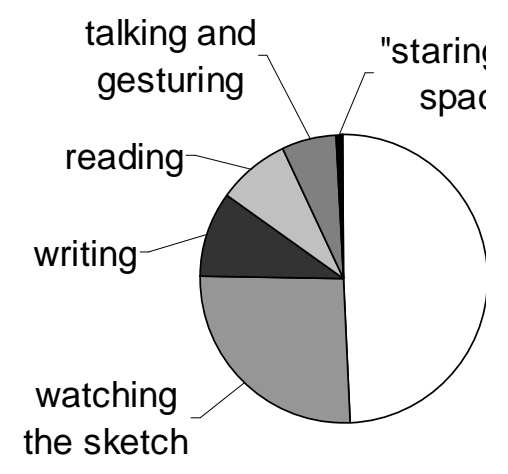

test person no. 3

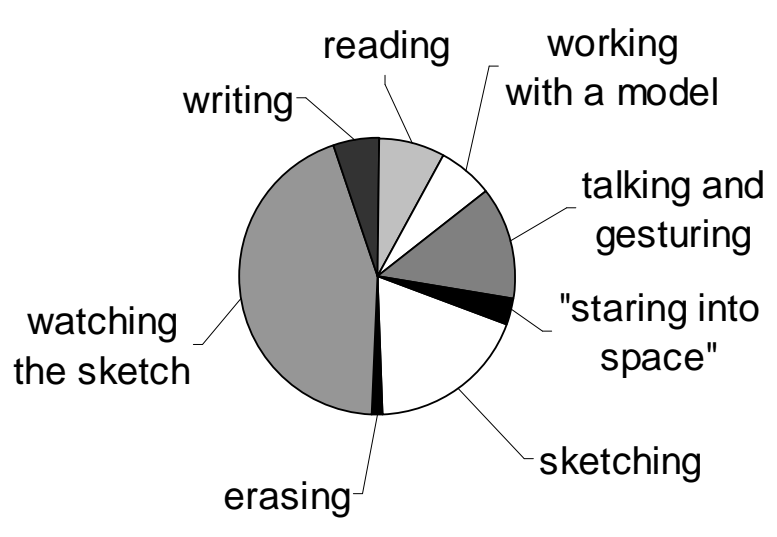

test person no. 39

Figure 4-8: Actions performed by test subjects no. 34 and 39 (corner cases) during the work on the laser welding task

Let us now take a closer look at the design activities that are carried out with the help of sketches. The observed phases of work with the sketch can roughly be divided into three different ones:

- problem analysis:

Only information given in the design task is depicted or interpreted, no solution approach is represented.

- solution development:

Conceptual solutions are depicted and altered.

- documentation of the solution:

The actual conceptual solution is no longer altered or supplemented, no more new conceptual solution elements are depicted.

In case of the laser welding task, $39 \%$ of the test subjects used a sketch to clarify the problem. Those persons depicted geometrical information that contained no new component. In the following chapter, we will see if this information mainly represents components given as boundary conditions or other information. 
For the laser welding task, the sketch was used significantly longer for solution development than in case of the barbecue grill task ( $\mathrm{p}<0.001$ ). Moreover the sketch was used significantly longer for problem analysis in case of the laser task than in case of the grill task $(\mathrm{p}<0.01)$. Figure 4-9 illustrates these findings. The more demanding a design task is, the greater the extent in which sketching is used for problem clarification and solution development.

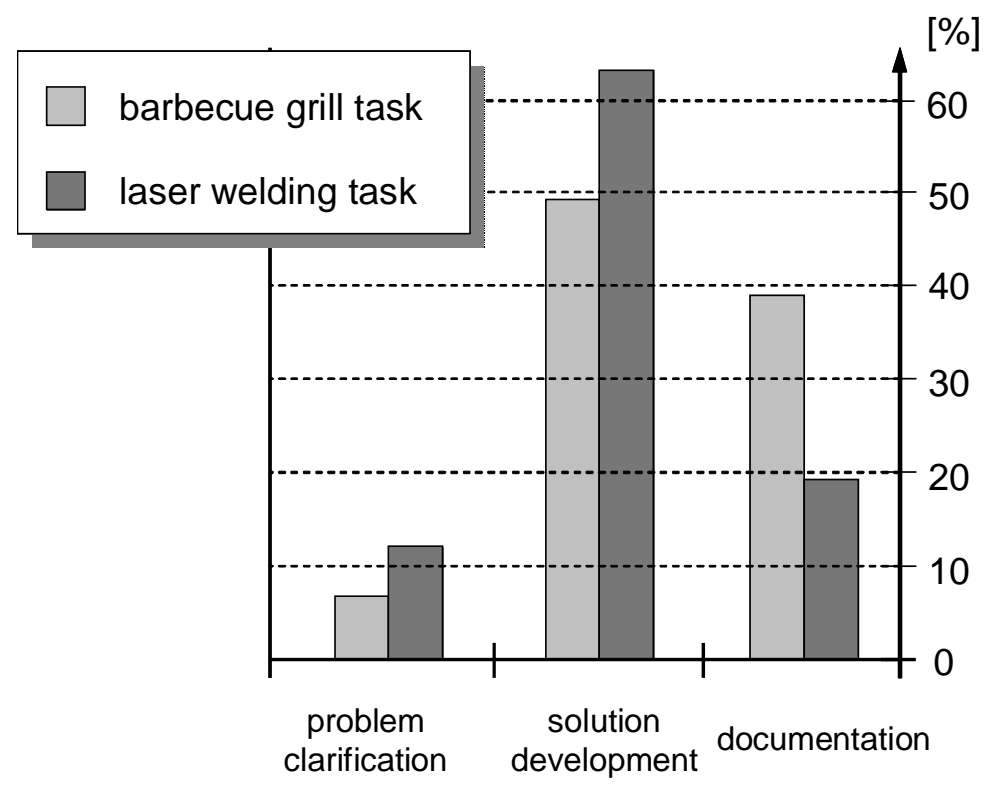

Figure 4-9: Observed phases of sketch work (average time shares referred to the total solution time)

Keeping in mind the description of problem solving processes by DÖRNER (1999B, "ARASKAM"-process, see page 22), problem solving processes may contain changes in the degree of abstraction. So predefinitions in a sketch, which are more abstract than visualgraphical depictions, might support the design process. Different modalities of depictions may be:

- visual-graphical

(illustrations that match the actual view on the object, which means that e.g. all visible edges are depicted, laws of perspective are applied)

- schematical

(reduction of actual shapes to more simple geometries, e. g. spatial objects are reduced to lines, joints are reduced to dots)

- symbolical

(e. g. arrows to symbolize motion, dotted lines to indicate symmetry)

- verbal

(words that are integrated into the geometrical context of the sketch) 
We will discuss these modalities in great detail in the next chapter. Figure 4-10 shows examples for such depictions from sketches that have been made by the test subjects during the experiment.

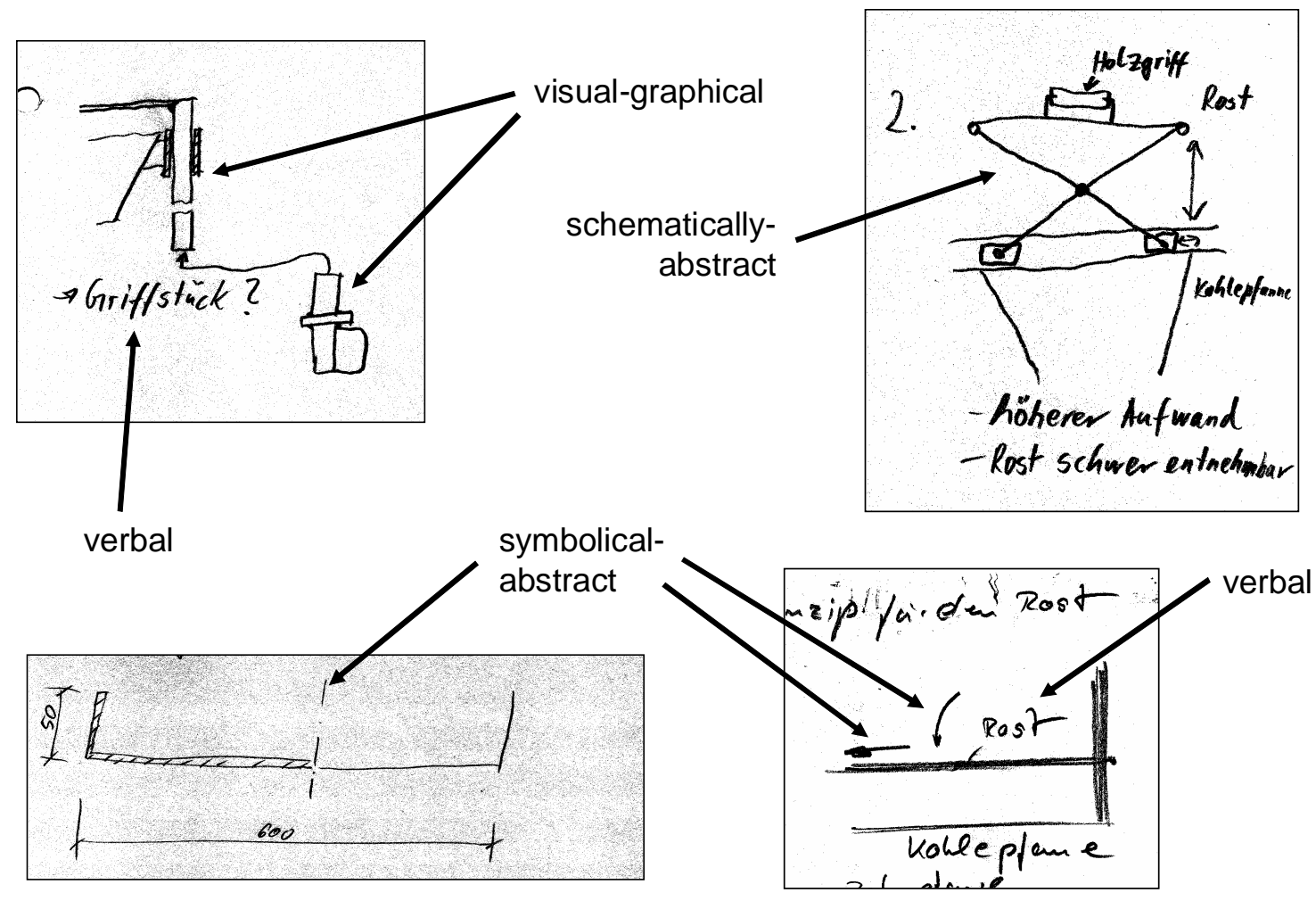

Figure 4-10: Exemplification of different depiction modalities

The sketches of all test subjects were analyzed with regard to the modality of depictions. Table 4-3 (upper four rows) shows the percentage of test subjects that used a specific depiction modality at least once during the design process. In case of the laser welding task, all test subjects used symbolical depictions. Most persons used at least three different depiction modalities (78\% in case of the barbecue grill task and $97 \%$ in case of the laser welding task). 
Table 4-3: Rate of test subjects that used certain depiction modalities and information categories at least once

\begin{tabular}{|c|c|c|}
\hline & barbecue grill task & laser welding task \\
\hline visual-graphical & $98 \%$ & $95 \%$ \\
\hline schematically-abstract & $48 \%$ & $69 \%$ \\
\hline symbolical-abstract & $82 \%$ & $100 \%$ \\
\hline verbal & $83 \%$ & $92 \%$ \\
\hline geometry & $100 \%$ & $100 \%$ \\
\hline motion & $41 \%$ & $93 \%$ \\
\hline
\end{tabular}

It has shown that for the laser weld task, about $73 \%$ of the test subjects used schematical depictions during solution development. Moreover, $7 \%$ did even use only schematical depictions to complete a conceptual solution for the task. All test subjects used symbolic elements within the sketch and $89 \%$ used written terms.

Furthermore, Table 4-3 (lower two rows) shows how many test subjects depicted certain information categories. Both design tasks implied kinematic problems, so the geometry of components and the kinematic behavior of components stood to reason as information categories. It is not surprising that in case of both tasks, all test subjects depicted geometry. In case of the laser welding task, about $93 \%$ of the test subjects depicted kinematic properties of the solution concept at least once. In case of the barbecue grill task, the rate was lower, but still came to $41 \%$. The motion of parts was depicted for example by arrows and curves.

In the following case study, we will further examine the depiction modalities, as well as the information categories within sketches. From these findings, we will make an attempt to conclude their meaning for the creative sketching process.

\subsection{Depiction Modalities and Information Categories in Sketches}

We have seen that designers do not sketch for conceptual design in order to represent technical solutions as photorealistically as possible. The results presented in the previous chapter show, that schematical depictions are used rather often. Moreover, words are used in a sketch to represent information that may be as abstract as language permits. The same applies 
to symbolical depictions: Again, all levels of abstraction may be used that are provided by the symbolical system. In the following paragraphs, we will carry out a case study to investigate the information modalities in more detail.

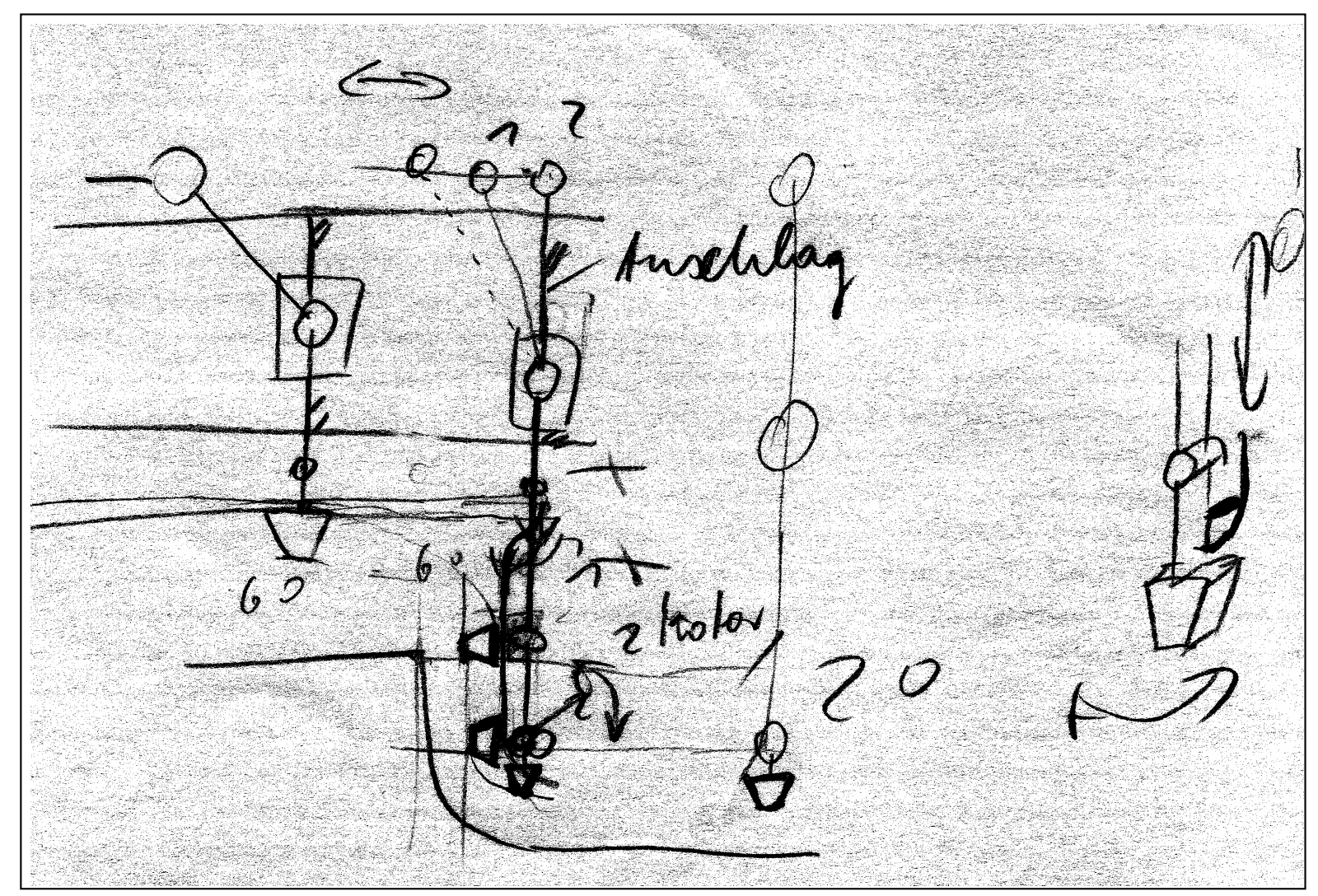

Figure 4-11: Overall sketch (left) and detail sketch (right) from test subject no. 17

Let us regard sketches from test subject no. 17, a student of mechanical engineering. Figure 4-11 shows the whole piece of paper with an overall sketch on the left side and a detailed sketch on the right side. The sketches were made during the phase of solution development. They present the approach to a conceptual solution that already enables a part of the desired motion of the laser. 


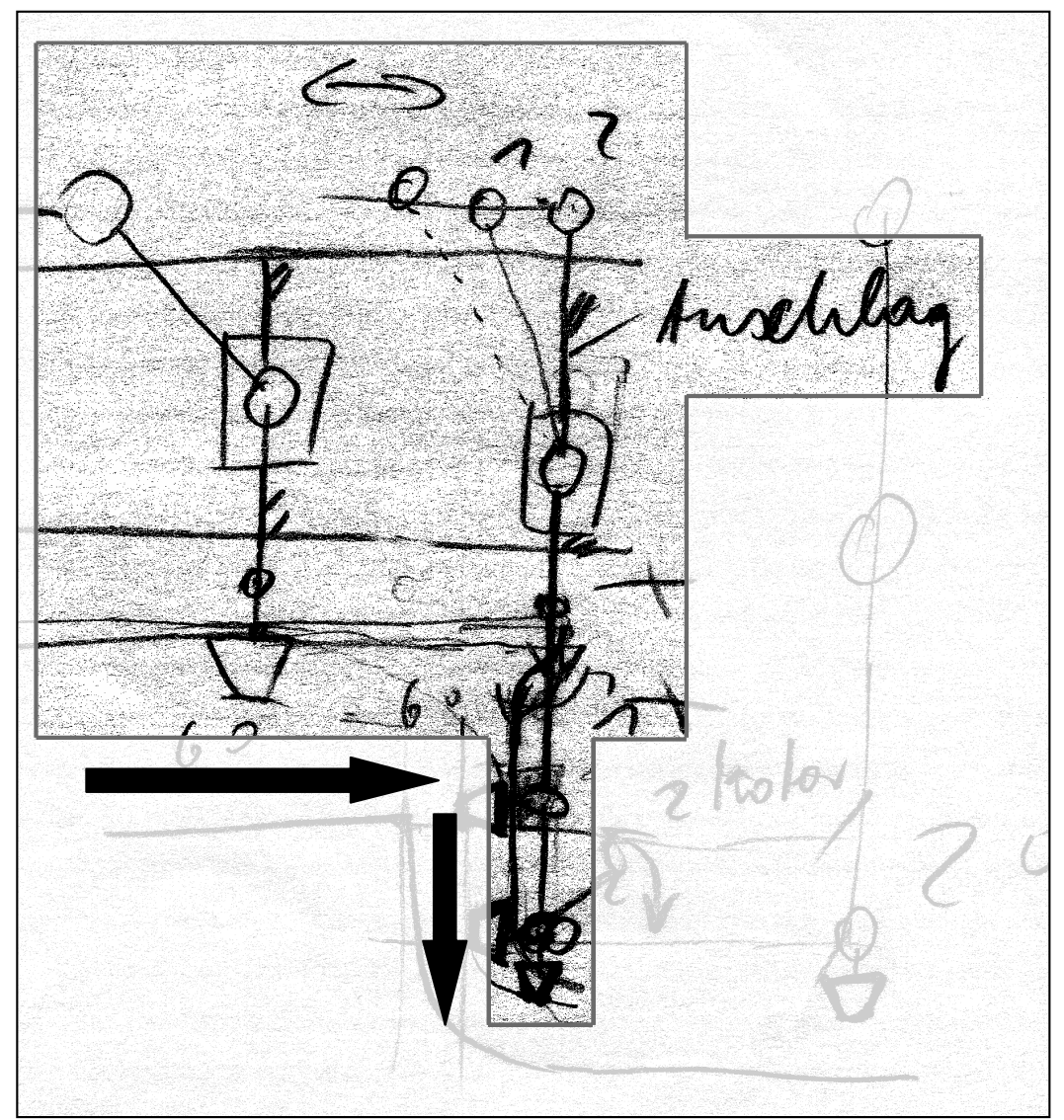

Figure 4-12: Section of the sketch that represents the device for horizontal and vertical motion

At first, we will explain the solution principle that was chosen by the student ${ }^{1}$. Figure 4-12 highlights the representation of that part of the device, which enables a horizontal and a subsequent vertical motion of the laser (represented by the black arrows in Figure 4-12). The functional principle that underlies the solution approach is illustrated in Figure 4-13. From state 1) to state $2 \mathrm{a}$ ), the whole device moves horizontally because it is guided by two rails (represented by two horizontal double-lines) in which the device rolls with the help of two wheels (two black dots). The device is actuated horizontally at the upper left bar (the actuation is represented by the grey arrow A) and the resulting motion of the laser (grey arrow $\mathrm{M})$ is horizontal likewise. This motion phase is completed as soon as the device bumps against the limit stop ("Anschlag" in German). It is interesting that this element is only represented in the sketch by the word "Anschlag". Yet, is not depicted - we will get back to

\footnotetext{
${ }^{1}$ The explanation was extracted from the interview with the test subject that was carried out directly subsequent to the experiment. The person was asked to describe the design process in own words and explain all technical solutions that arose along the process.
} 
this subject soon. The limit stop inhibits the horizontal motion of the device, but the actuation continues. So in state $2 b$ ), the vertical slide bearing is activated (represented by two short horizontal lines on both sides of the long horizontal line). The laser moves downwards (grey arrow M) and the two joints (circles) transmit the horizontal actuation (grey arrow A) until it reaches state 3 .
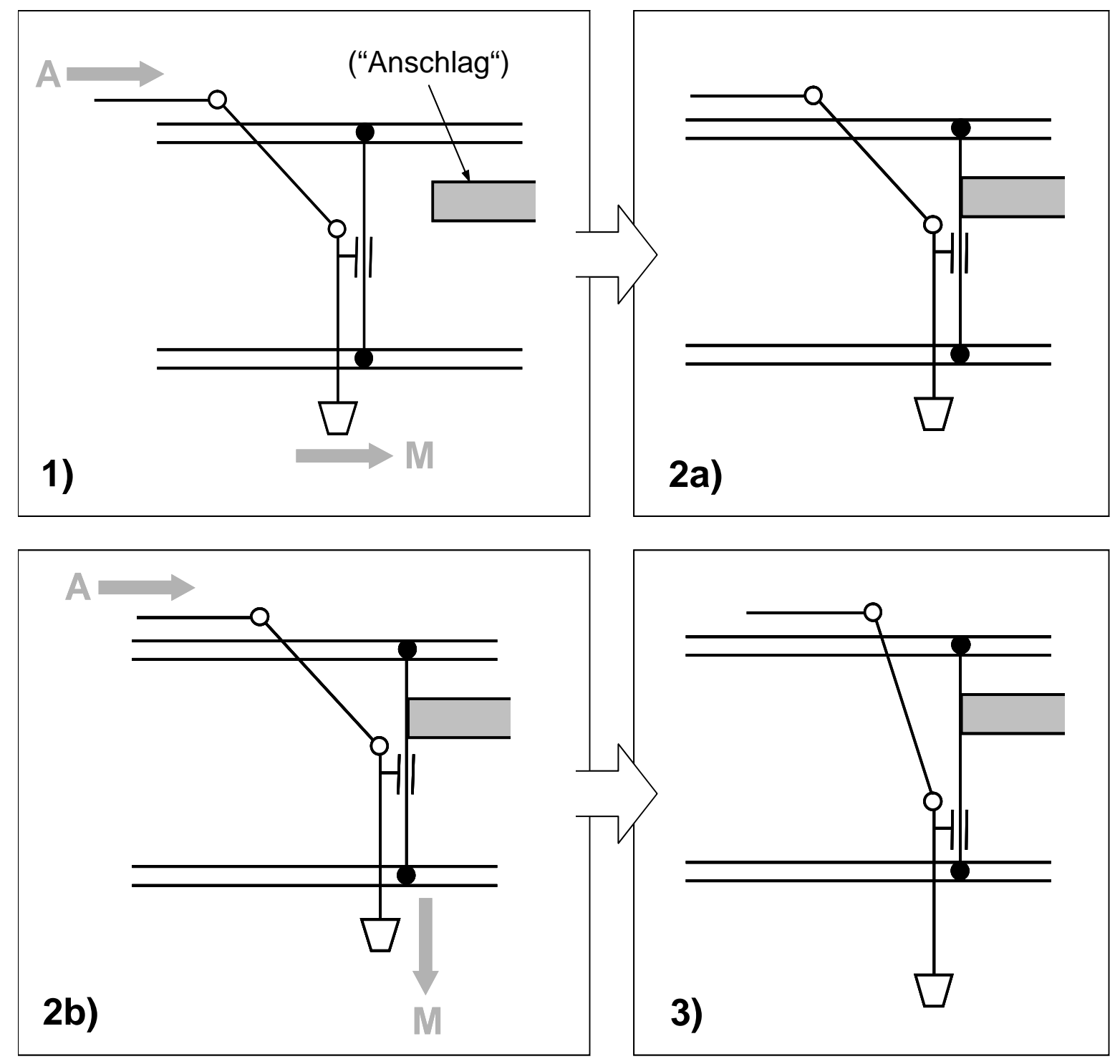

Figure 4-13: Functional principle that enables the horizontal and vertical motion of the laser 
In the first instance, the description above shows that very complex technical systems can be represented in sketches by rather few lines. Considering the overall sketch once again (see Figure 4-14), it shows that not only technical components are represented. The processes resulting from the behavior of the components, such as motions, are represented as well. Components are drawn repeatedly to represent different states of a motion (one example is labeled by 1, 2 and 3). Symbols, such as arrows, indicate that certain components move in a certain direction (A). In mechanical engineering, the design of a technical device includes designing a technical process since providing a desired behavior (indirect attribute) is the superordinated goal of design. In chapter 3.2.1, we have seen that complex kinematics are characteristically for many design task in reality (see page 47). It seems as if the student has dealt with the complexity by depicting not only the technical components, but their kinematic behavior as well. In the previous chapter, we have seen that (at least in case of the laser welding task) motion was depicted rather often (see page 83).

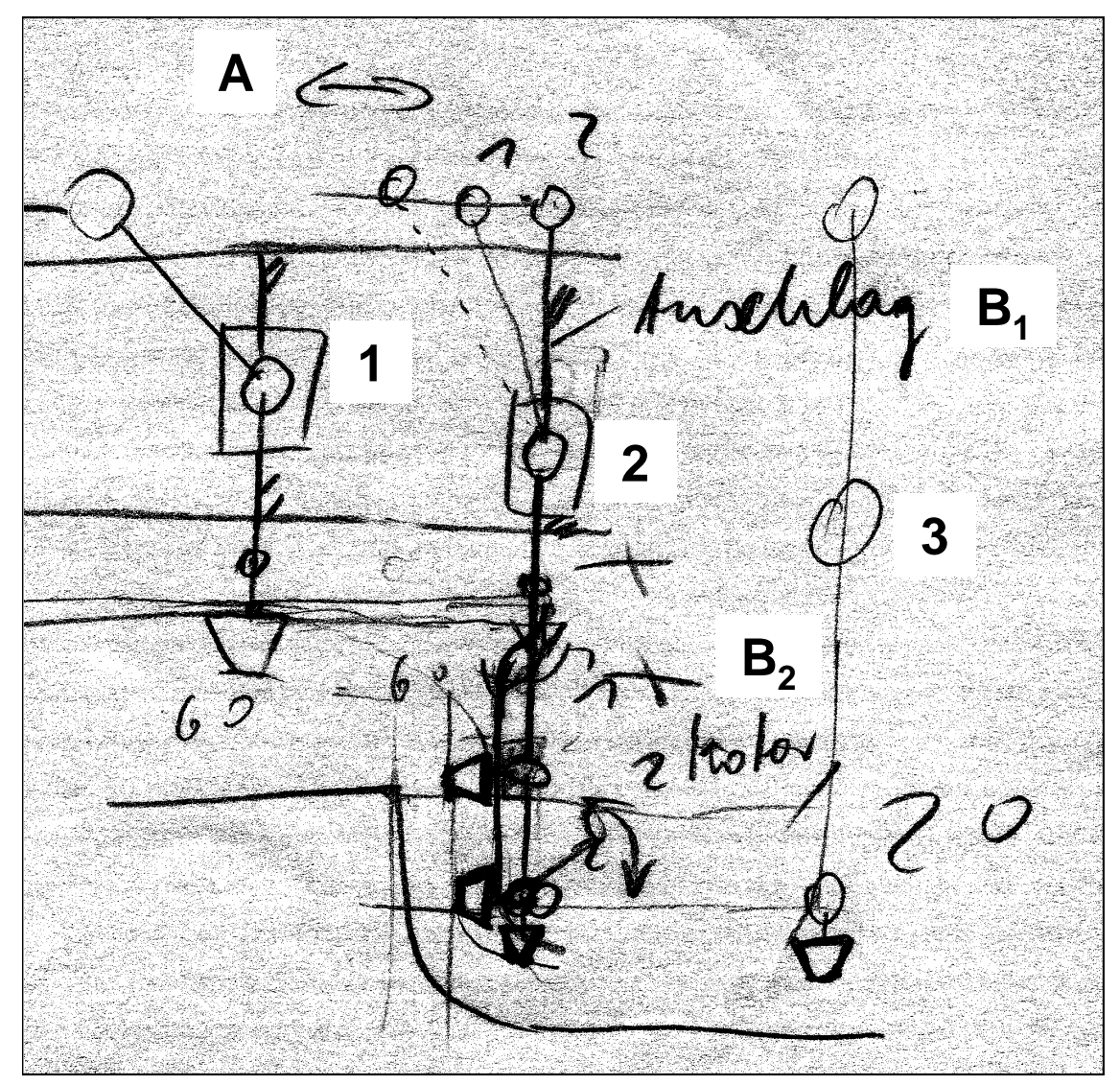

Figure 4-14: Different categories of elements used in the conceptual design sketch

However, in the previous chapter we have also seen that the representation of components is not limited to the use of depictions, but verbal and symbolical representations are used, too. Words do not only label geometrical elements that are already depicted, but they may 
represent a component entirely. In this sketch, "Anschlag" $\left(\mathrm{B}_{1}\right)$, as well as "Motor" $\left(\mathrm{B}_{2}\right)$ represent components that are not depicted explicitly. By using textual predefinitions, a designer can take advantage of the whole variety of different levels of abstraction that are provided by language. The designer does not yet need to specify a component geometrically. By using symbols, a designer can represent components on similar levels of abstraction (i.e. circles represent joints without further specification of the special kind of joint). Still, symbols, in contradiction to words, often give a slight visual impression of the component's shape.

Besides geometrical elements, a sketch can provide a designer with symbols and written terms representing components, as well as their functional attributes on different levels of abstraction. Furthermore, the elements in a sketch have relations to each other; a number of lines may form a shape and a number of components may represent an assembly. DÖRNER (1999B) points out the meaning of abstraction and concretion for problem solving in conceptual design, but he assigns these processes mainly to verbal information. A sketch provides external representations for information chunks on different levels of abstraction that a designer might have in his mind during design. In this regard, the sketch can provide memory relief for the designer's mental process. Still, in this case the sketch is a rather "simple" memory relief, similar to notes put down on a memo pad. DöRNER (1999A) describes problem solving as an inner dialogue with oneself. In this regard, the sketch would only be the intermediate storage for some single terms from that dialogue. The "questions" and "answers" that actually make up such a dialogue would be formed in the mind. We suppose that the sketch is capable of even more than just representing single terms in a clear and easily accessible way.

Even those elements that show the specific shape of a component are not purely concrete representations, they provide a certain level of abstraction referred to the future component. In the previous chapter, we have labeled those elements schematical (see page 81) and we have seen that they appear rather often in sketches (remember that $7 \%$ used no visual-graphical, but only schematical elements to depict the geometry of their barbecue grill solution). In the sketch above, almost every element is depicted as a line, which is a one-dimensional depiction, since there are only few closed shapes in the sketch. In reality, all these components will be spatial objects, but in the sketch, this spatiality is not determined. The elements in the sketch only define the extension of the components in one main dimension; the other dimensions are left open. The bars that are represented in the sketch may have any kind of profile in future - it is just not specified yet. So elements in a sketch may be abstract in a sense that they omit certain classes of geometric attributes, such as certain spatial dimensions. It may be argued that a single line is a symbol for a bar, but the author does not agree to this argumentation. Still, a line contains aspects of symbolism as well, but the symbolic meaning of a line depends on more than just the line itself. In the next chapter, we will see that the same line may once represent a track (which can still be seen as a kind of bar) and then represent a belt (which is certainly no bar). 
The detail sketch (see Figure 4-15) shows that this abstraction can be abrogated stepwise. In Figure 4-13, we have seen the functional principle that causes the horizontal and the vertical motion of the laser. Yet, the laser also has to rotate between those motion phases in order to enable the welding process during the horizontal motion. This problem was solved by a stud that is connected to the laser. The laser is connected to the rest of the device by a joint and may rotate therefore. The stud glides along a guide slot and the shape of the slot causes the stud to move and therefore the whole laser rotates around the joint. This solution was only possible by specifying the shape of device in the third dimension. Likewise, the detail sketch is drawn 3-dimensionally to reveal the spatiality of the device.

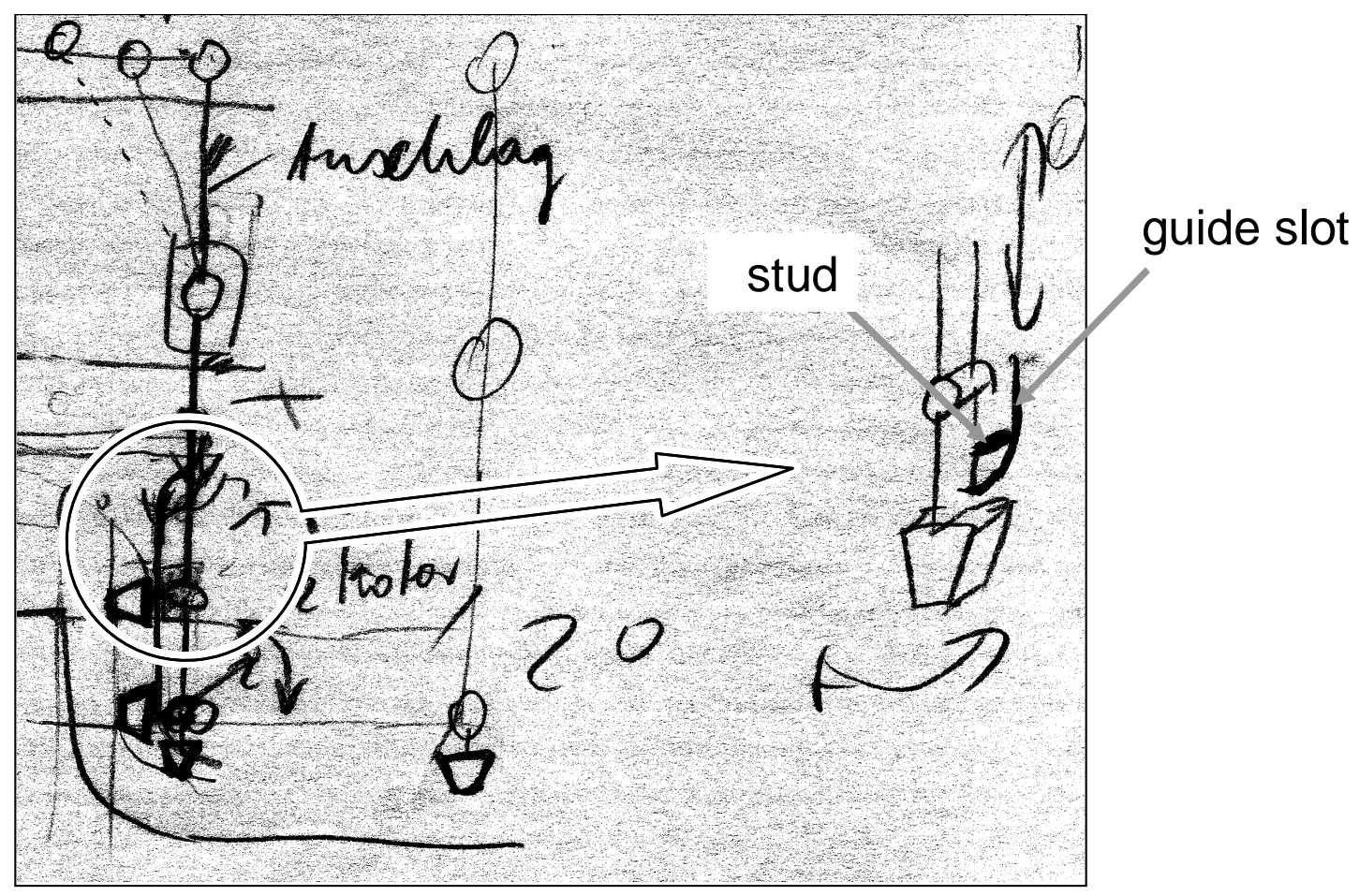

Figure 4-15: Detail sketch drawn in perspective

The abstraction discussed above consists in omitting certain classes of attributes. Such abstract predefinitions represent a variety of concrete predefinitions since the attributes that were left open may be specified in any way. There is another, different kind of abstraction that does not omit certain geometric attributes completely. It rather narrows down the range of possible specification for a certain attribute, yet it still leaves a scope. This is the case, when the shape of a component is specified vaguely. Roughly drawn shapes can represent a variety of specific shapes. The recognition of this variety depends on the contextual knowledge of the viewer. A viewer unknown to the context might interpret a roughly drawn, wavy line, as a concrete shape that is meant to be exactly that wavy. 
Figure 4-16 shows the element of the sketch that represents the sheet metal given in the design task. It may be interpreted as the representation of a component that is as wavy as the original line in the sketch. However, the element may as well be interpreted as shape A), which is clearer and sharper than the sketched line. This conclusion might be drawn if one regards the waviness as a lack of accuracy in sketching. Still, in this case, it was actually shape B) that was represented by this element. So not only the line was drawn wavier than the actual shape, but also the partial elements were drawn in different proportions than in case of the actual shape. In this case, there are at least three different ways to reasonably interpret the shape of the line.
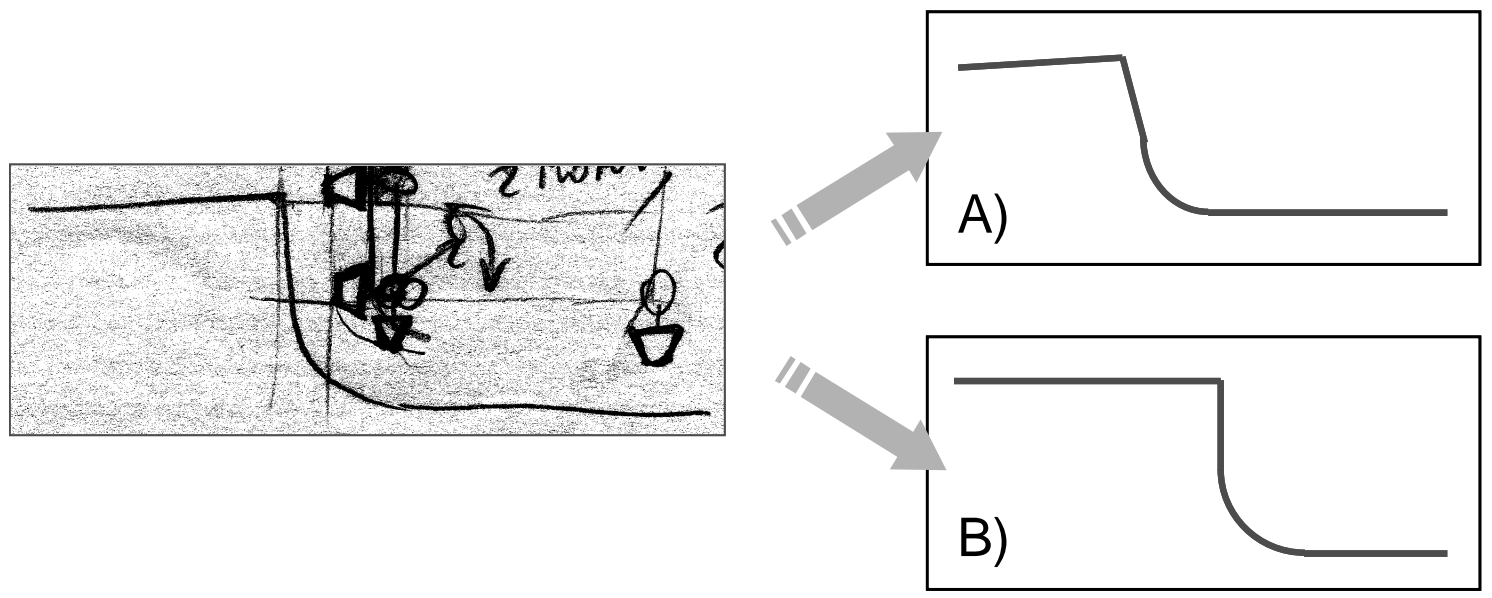

Figure 4-16: Roughly drawn line and possible ways to interpret it

This kind of vague predefinition described before may be seen as an abstract elements in certain aspects. Such an element may be interpreted by a viewer as a representation of one certain concrete shape at one time and as a different one at another time. So this element contains a variety of possible concrete shapes. It may be that a viewer does not only switch between different interpretations, but that he is even able to see this variety. Keeping in mind the definition of abstraction ${ }^{1}$, the representation of a variety of concrete terms is a characteristic of abstract, superordinated terms in general. Although in the case of graphical elements, the attributes that are omitted (respectively narrowed down) can hardly be named by words, the effect may be just the same. If one tries to describe such a shape in words, this person will probably end up in either describing it exactly, which results in an extensive text, or in using terms such as "somehow", "sort of" and "thereabout". Still, the shape cannot be named by using one term and simultaneously reflect its superordinated character. Visually perceiving such a shape may achieve just that: one can perceive the whole shape at once and still "see" the variety of specific shapes represented by it. Thereby, an abstract graphical

\footnotetext{
1 DOERNER (1975) defines abstraction (similar to the definitions used in engineering design research) as "omitting inessential attributes respectively accentuating essential attributes" (page 7 in this thesis).
} 
element may function in the same way as an abstract verbal term, although such a graphical element does not have a direct verbal counterpart. It may be possible that designers use such a abstract graphical predefinition in a calculated way just when they are not certain about the future shape yet. Still, this does not necessarily need to be a conscious behavior. Due to the experimental setting used in this analysis, it is not possible to derive any statement whether designers intend to represent abstract, vague element or if they just sketch inaccurate. Still, the verification of this question would provide insight into the creative generation of shapes. Even if it shows that designers do not use abstract, vague elements on purpose, it may be worth it to think about teaching such procedures in order to improve creative sketching. Likewise, it might be necessary to practice the recognition of vague shapes as abstract predefinitions.

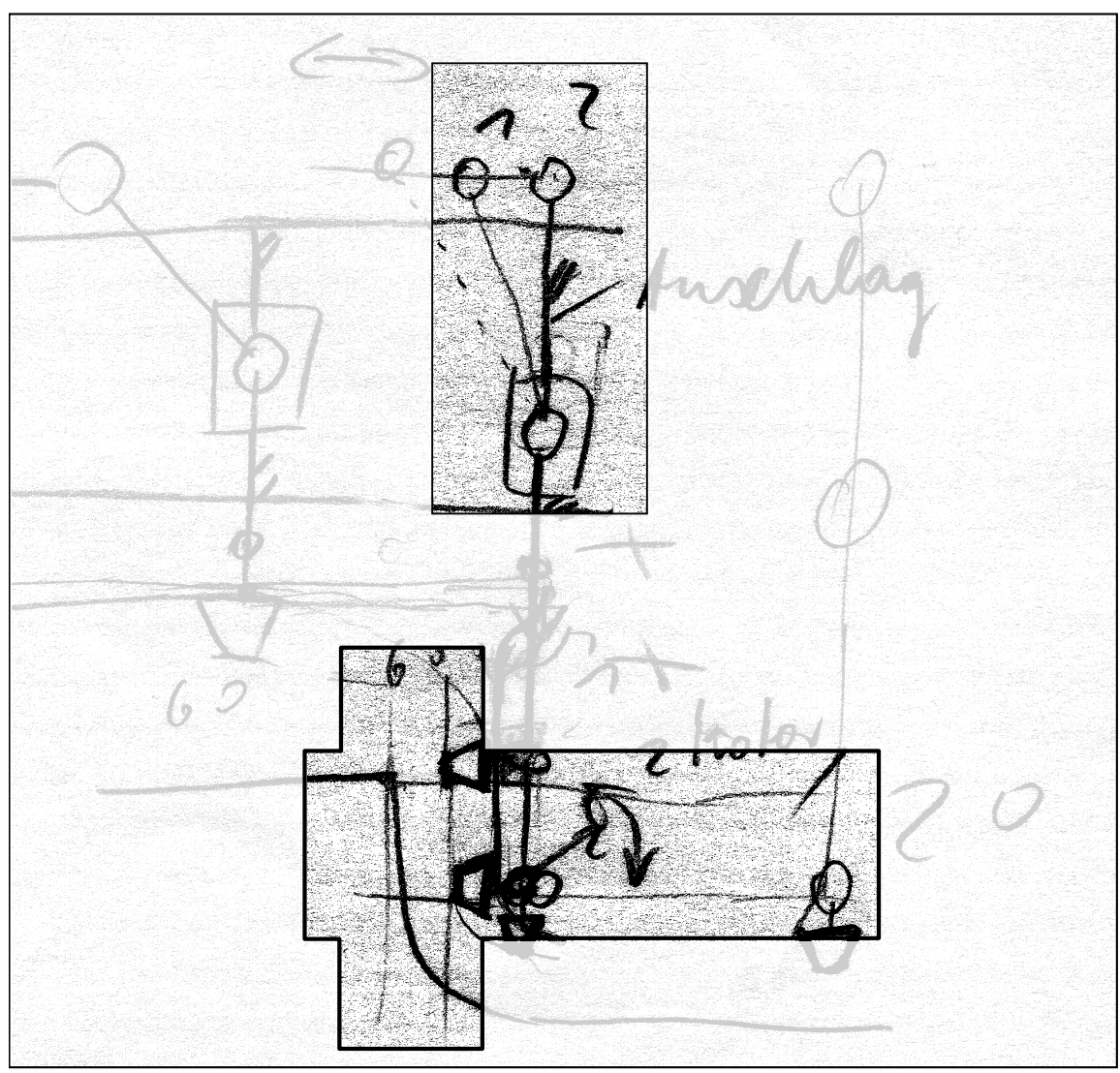

Figure 4-17: Auxiliary elements and numberings to express spatial and temporal relations

Generally considered, a sketch puts graphical, symbolical and verbal terms on different levels of abstraction into a spatial-relational context. This context is more diverse than the context that language can provide. Terms, such as "above", "below", "left from" and "right from" can be specified by the position of elements on a sketch. Still, these relations can even be represented by special elements. In the sketch, the student uses ancillary elements, such as 
thin lines (see Figure 4-17, lower section), that indicate that certain elements are on the same horizontal or vertical level with others. These lines do not represent objects that will be manufactured when the device is produced in the end. These lines also do not represent indirect attributes such as the behavior of a technical system. They "explain" or even "emphasize" the direct geometrical attributes of the device. So besides the geometrical relations between the elements on a sketch that emerge automatically, a designer can use special elements to express such relations. When processes are represented, then even the temporal sequence of process steps can be depicted. Different states of a motion process can be annotated by numbers that indicate the order of the states (see Figure 4-17, upper section).

Let us now recall the consideration of problem solving as a dialogue with oneself (DÖRNER 1999A, BERTAU 1999). We have already applied the communication model from SHANNON \& WEAVER (1949) to such a soliloquy via external media. In the following paragraphs, we will analyze the "language" that may be used for such a dialogue. We will indeed apply some basic concepts from linguistics to a sketch, yet we will not go into detail according to this complex domain. In linguistics, phrases are small groups of words which are the basic elements of sentences. Linguists differentiate between different kinds of phrases (e. g. CRYSTAL 1995, pp. 94), such as:

- nominal phrases

A nominal phrase is a part of a sentence that refers to a person, object or event, which may exist in reality (e. g: "George", "the black dog", "they")

- verb phrases

A verb phrase refers to actions and processes. It usually appears together with at least one nominal phrase to indicate the object of the action or process respectively. Some verb phrases only need one nominal phrase (e. g. "is standing", "went fast"). Thereby, those phrases express something about the object, such as its behavior. Other verb phrases need more than one nominal phrases (e. g. "carries", "is giving") and thereby the action/process implies a relation between different objects and subjects.

- prepositional phrases

A prepositional phrase expresses spatial or temporal relations between objects (e. g. "above", "under", "besides", "on the same level as", "before", "after"). These relations may be symmetrical, which means that the nominal phrases can be exchanged and the meaning stays the same. Such a prepositional phrase does not have a specific direction ("A is on the same level as B" means the same as "B is on the same level as A"). Others are asymmetrical, so they indicate a directional relation (" $\mathrm{A}$ is above $\mathrm{B}$ " is different than " $\mathrm{B}$ is above $\mathrm{A}$ ").

If one might consider the elements in a sketch as a sort of graphical language, then functional symbols (such as arrows) would be "graphical" verb phrases. Those elements representing components would be "graphical" nominal phrases then. We have already discovered prepositional phrases such as the thin lines used as auxiliary elements. Especially these elements urge to consider sketches as a graphical language, since these elements are actually not necessary to represent geometric information. Spatial relations, such as above, below, left 
from and right from are provided automatically by the position of elements within a sketch. Such ancillary elements are the expressions of relational information that is given anyway, yet they emphasize this information. Even directional relations can be represented, such as the indication of temporal relations by numbers. Taking graphical abstraction by rough shapes and contextual abstraction by words and symbols into consideration, the information content of a technical sketch can be enormous and on a high level of abstraction. Complex sketches can literally tell "technical stories" to a viewer who is familiar with the technical context of the sketch.
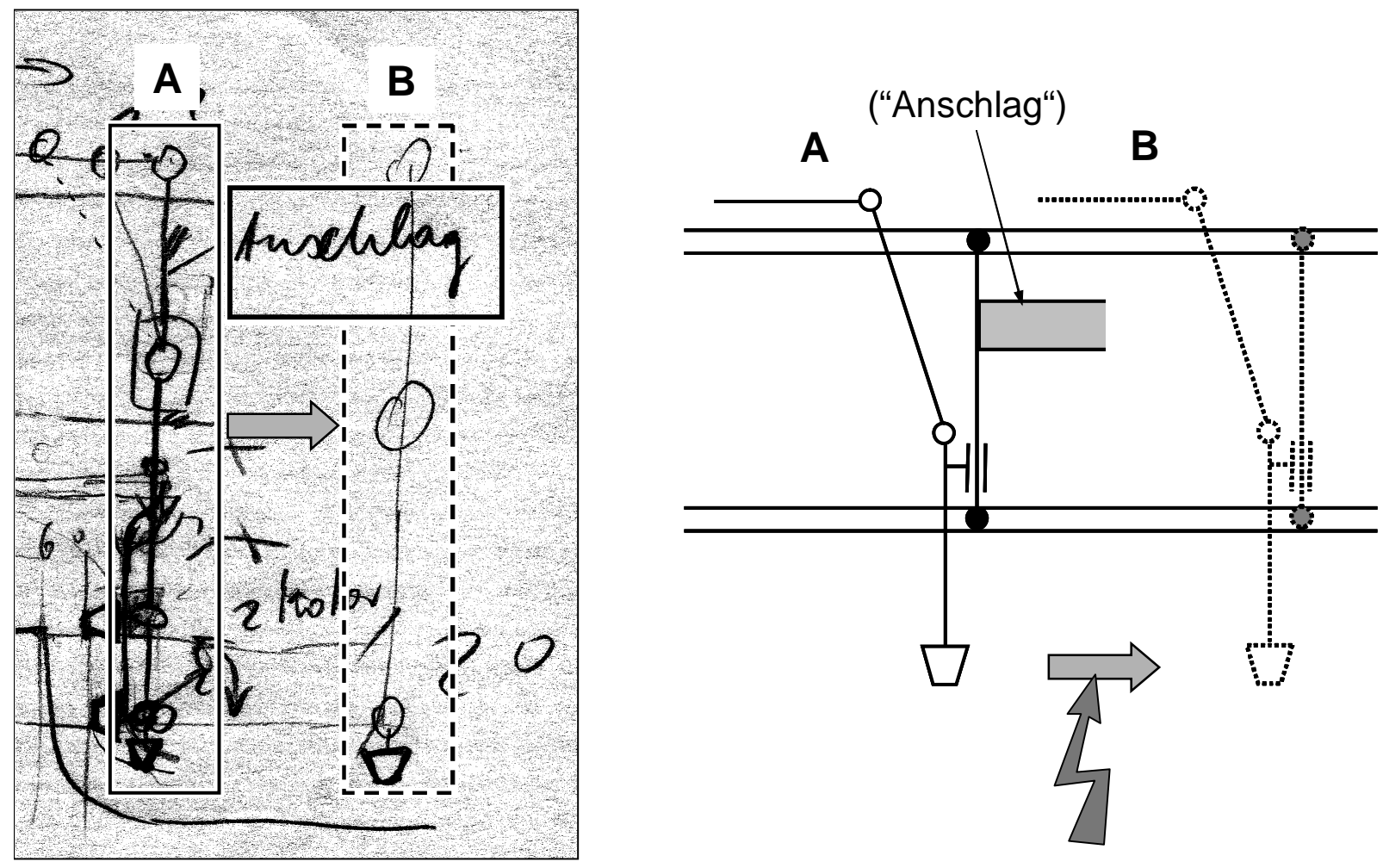

Figure 4-18: Representation of a contradiction in the conceptual sketch

The stories that are told by sketches can even be contradictory and irrational as shown in Figure 4-18 shows. The sketch contains a contradiction since state A cannot be transformed into the terminal state B because of the "Anschlag" (limit stop) in-between. Still, this obstacle is necessary in order to translate the horizontal actuation into a vertical motion during the process step before. Still, the student has depicted the terminal state, although it cannot be achieved (yet). It may be supposed that the student has been aware of this contradiction. The "impossible" terminal state is depicted very different from the other states. It is represented by thin lines, which may be regarded as an indication of a provisional predefinition. The contradiction may not be seen as a faulty solution approach, but as the representation of the problem. Such a contradiction can be on a high level of abstraction and may generate tenseness for the viewer, being the equivalent to a question, such as "How can that ever 
work?" This way, a graphical dialogue, conducted by the designer with "himself" via sketches can be provoked, initiated and even enforced.

Furthermore, this example suggests that the line thickness is a significant characteristic of a depiction. The last motion state, which is actually irregular in the technical context of the sketch, is drawn by thin lines. The use of thin lines may be an indication for a provisional predefinition.

In the last chapter, we have seen that in case of the laser welding task, about $40 \%$ of the test subjects used sketches for clarification of the design problem. Let us now briefly view such a sketch in order to find out which kind of information is depicted for problem clarification. Figure 4-19 shows the very first sketch that was drawn during the laser welding experiment by test subject no. 34, a designer from industry with 21 years working experience. The designer depicted the sheet metal that was supposed to be welded (which was given in the task description) and then developed the necessary motion of the laser. He sketched the laser in several characteristic positions and determined the curve that the laser had to move along.

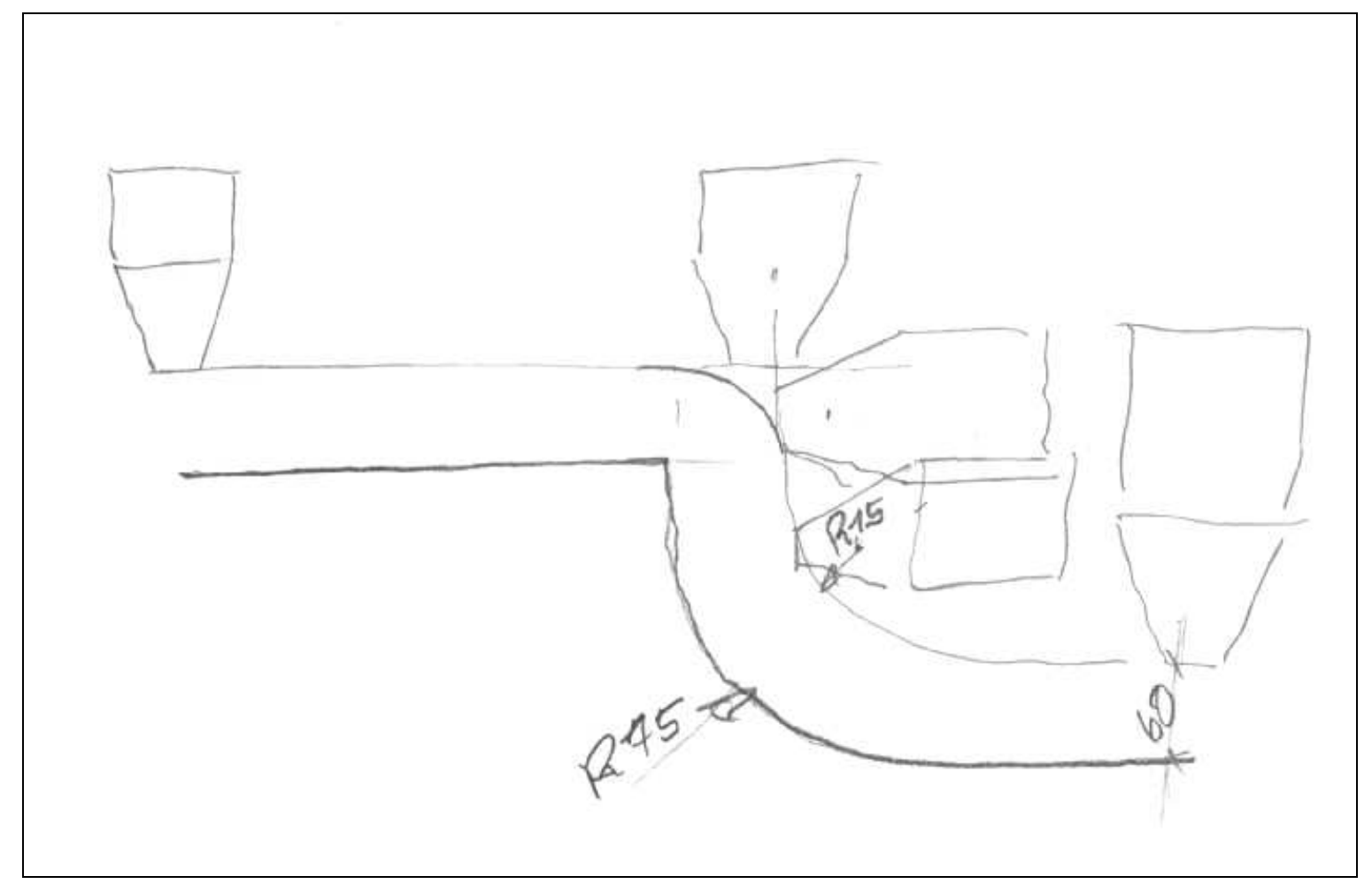

Figure 4-19: Sketch that was drawn during problem clarification by test subject no. 34

In this case, the designer developed and depicted the behavior of the future device even before he depicted the first idea about how the device might look like. It may be supposed that he did not even have an idea about the functional principle of the future device yet. So in this case, kinematic information is not represented as the result of the analysis of some existing solution's behavior - it rather represents the starting point of the search for such a solution. 
Figure 4-20 outlines this matter. The indirect attributes of the future device are depicted at first and then the direct attributes are deduced, so the succession of depiction can be headed from indirect to direct. This procedure corresponds to the "two-sided problem solving" approach (LEHMANN 1972, p. 51) which is suggested for the reduction of the search space (see page 21).

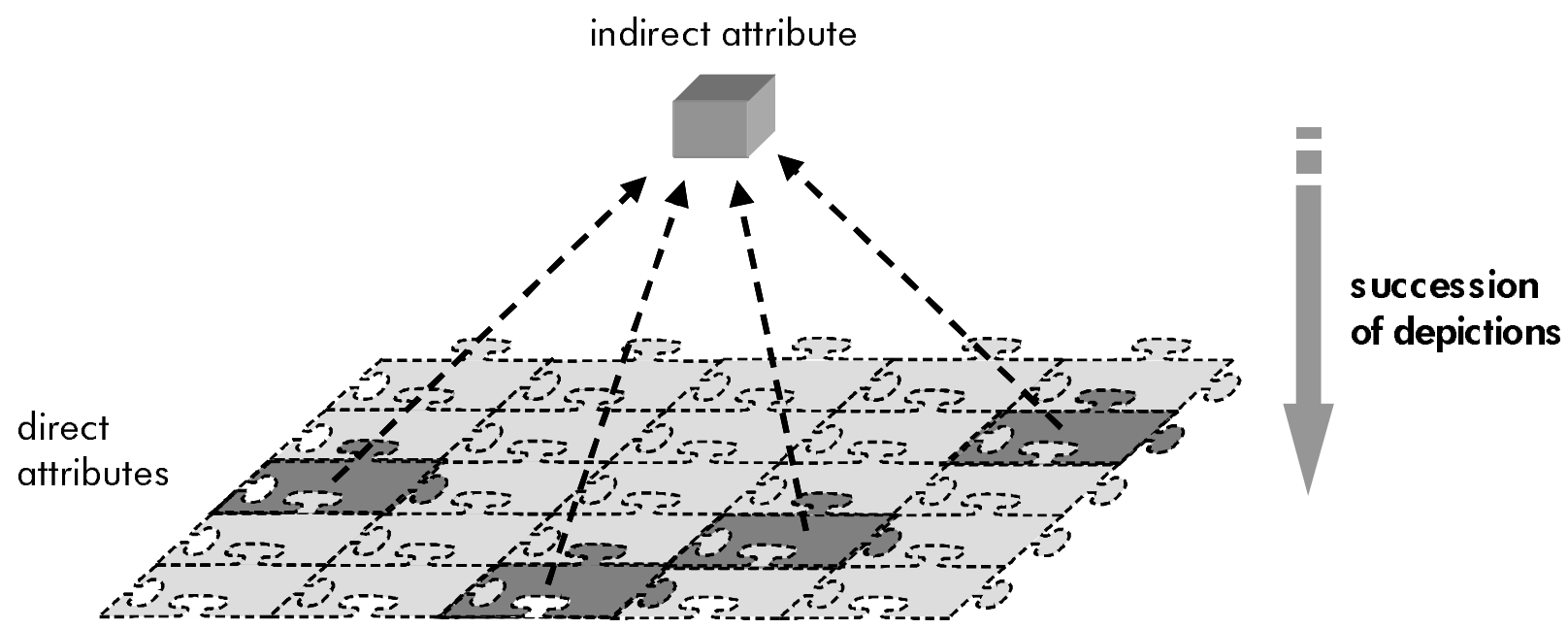

Figure 4-20: Schematic illustration of the development of direct attributes starting from the depiction of indirect attributes

\subsection{Perceiving Conceptual Design Sketches}

Previously, we have mainly discussed the content of a conceptual design sketch. Regarding the vagueness of shapes, we have already pointed out that the perception of shapes may imply an interpretation process that determines the information that is actually received from the sketch. Furthermore, we have seen that a sketch may contain a broad variety of information categories and depiction modalities at once. Geometry and motion is depicted with the help of graphical, verbal and symbolical elements on different levels of abstraction. We will now analyze in which way the perception of an emerging sketch over a period of time may alter the information that was initially depicted.

The following sequence has happened during the experiment of the student test subject no. 17 (the same person whose sketch was considered in the previous chapter). It occurred early within the experiment, shortly after a first solution approach was developed and just before the sketch we discussed in the previous chapter. 
The desired motion of the laser was given by the shape of the sheet metal as described in the formulation of the task. The student decided to achieve the motion of the laser by two tracks that were shaped just like the curve which the laser had to move along, as shown in Figure 4-21. The laser was connected to a "crab" that ran in these tracks. Thereby the laser perforce had to move along the shape of the tracks. This solution is comparable with the principle of a monorail or a roller coaster. The student sketched this approach by using simple curves that represented the tracks and the depiction of a "cart" with wheels, which represented the crab (drawn twice to point out its motion). The laser, connected to the crab, was represented by a simplified shape.

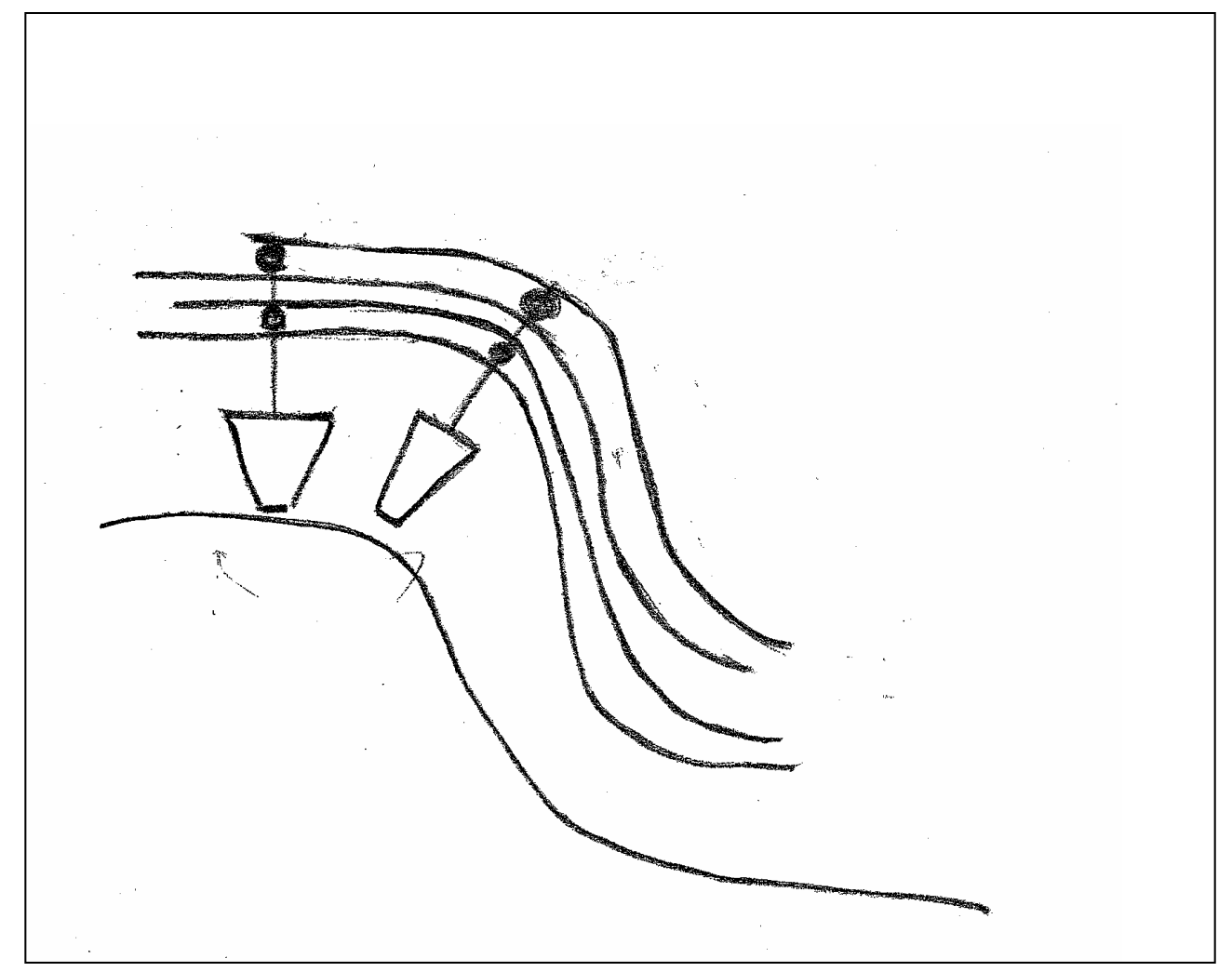

Figure 4-21: Sketch that represents the solution principle to realize a defined motion of the laser

Still, this approach only covered the problem of guidance for the laser. It did not cover the problem of actuation since the laser had to move with a certain speed along the tracks. To actuate the crab, only the linear drive was available and it provided a linear actuation. Therefore, this linear actuation needed to be transmitted into the rather complex motion of the crab. The student then clarified the actuation problem by sketching the speed vector of the crab's motion, represented by an arrow attached to the crab pointing in the direction of the motion. He then added the horizontal and vertical portion of the speed vector, forming a vector triangle (see Figure 4-22). 


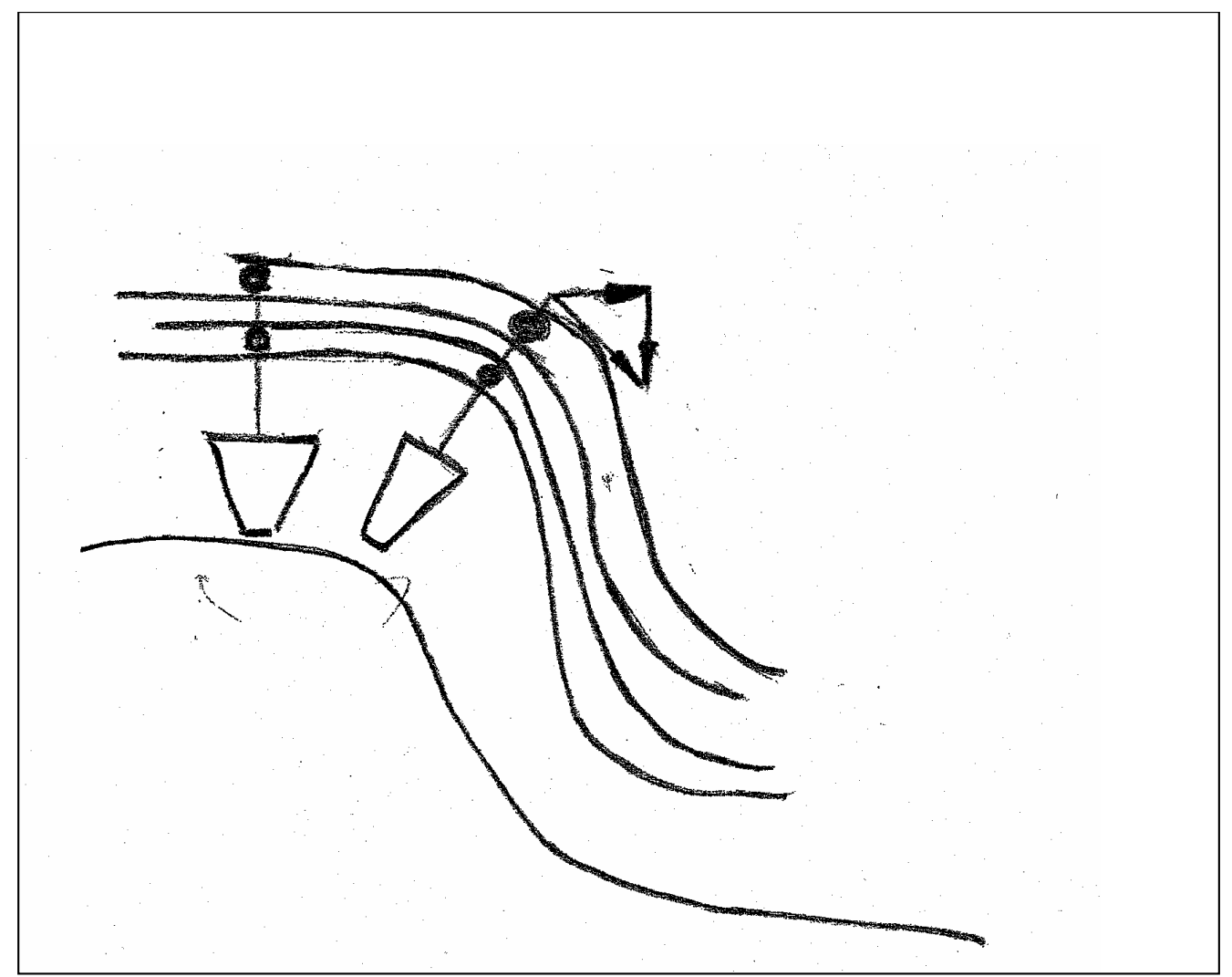

Figure 4-22: Sketch after the speed vector triangle of the crab was added

After considering the sketch (for a repeated time), he drew one of the track curves (the one that was closest to the vector of the crab) again right over the existing one and thereby intensified it, but did not alter its shape (At this point, it is important to mention that the motion of the student's hand along the track curve matched the direction in which the crab was supposed to move along the tracks). Afterwards, he intensified the track curve once again in a slow manner. Then, without removing the pencil from the sheet, he drew beyond the existing shape and completed the curve to a loop, as shown in Figure 4-23. He added pulleys, represented by simple circles, to some corners of the loop and attached the laser directly to the loop. 


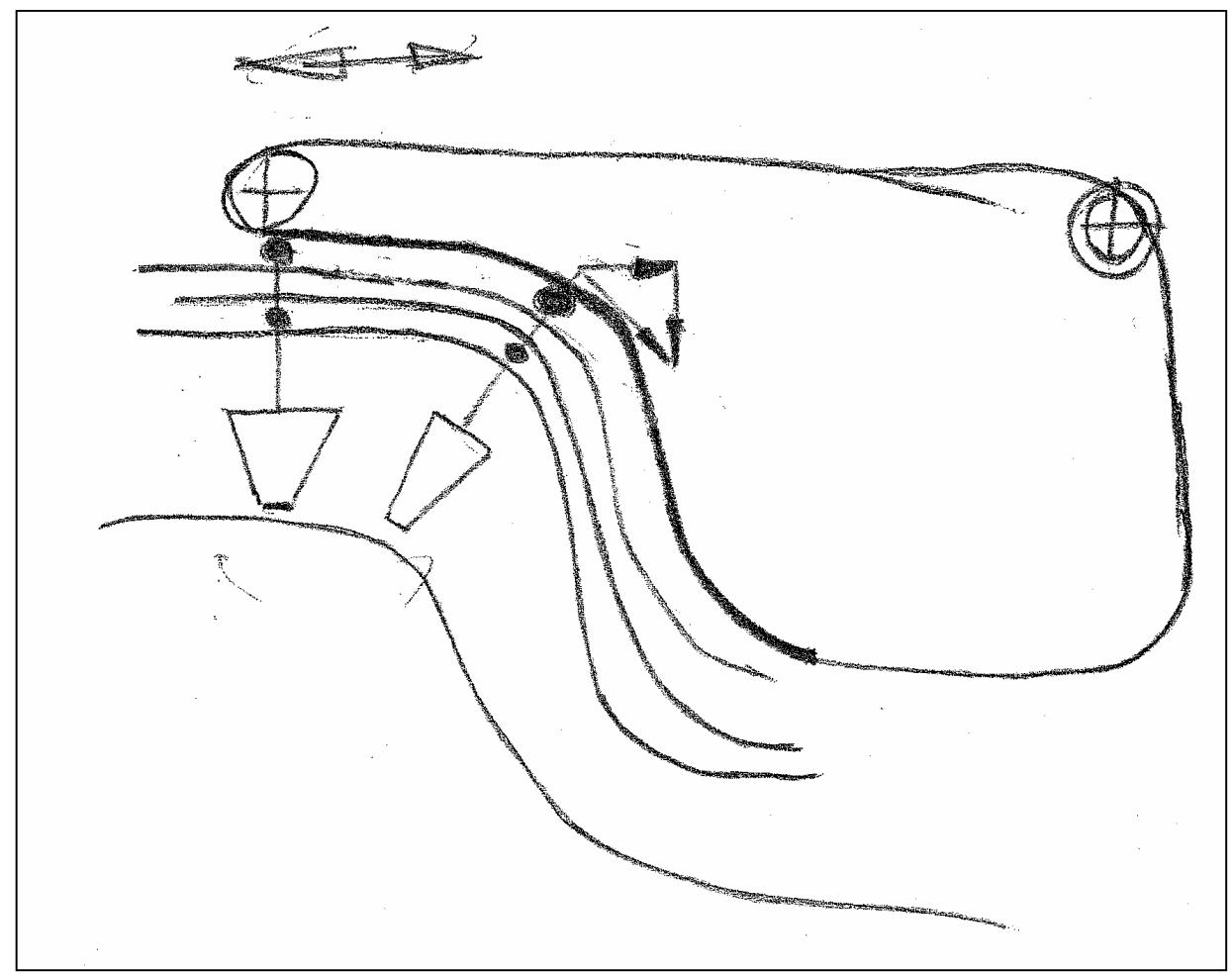

Figure 4-23: Sketch after the solution principle has been changed into a circulating belt

Therefore, just by adding few lines, the student altered the solution principle from a crab running in tracks into a circulating belt with the laser attached to it. Since a belt is not stable in shape, it is forced by the pulleys to move along the desired curve. Within the described sequence, the whole design concept was changed significantly. So what might have happened within these minutes?

Premising that the change of concept has been initialized by the sketching process, there are two approaches to explain this process which correspond with findings from design literature. One approach is to assume that the speed vector of the crab and the track curve have been attended together and have been correlated. Each element has been drawn independently and without a direct, contextual relation to each other since the vector represented the motion of the crab. The student may have referred this vector to the track and interpreted the combination of both elements as a curved shape that moves "in itself". Such an object may be a belt or a chain. Furthermore, this assumption is corroborated by the fact that from all curves representing the tracks, the one that is closest to the vector has been chosen for modification (although all curves have the same shape). It is probable that a viewer can correlate elements more easily when they are spatially close to each other, since they may be interpreted as being just one shape. In fact, the vector almost touches the curve and is actually closer to the curve than to the crab. The discovery of this relation may have been actively supported by the 
student himself in intensifying the track curve. Thereby, he has highlighted this element since its contrast to the surrounding has been increased.

This approach to explain this sketching sequence corresponds with the "unexpected discovery" of relations between elements described by SuwA et al. (1999). Still, Suwa and his co-authors do not specify any alteration of a conceptual design solution, but only refer to the discovery of new shapes or of geometric attributes of shapes that were not noticed before. In the case we just discussed, the discovered relation has altered the conceptual meaning of the sketch radically. This might be due to what has been represented in this sketch. Even the few elements in this sketch contain enormous information beyond sheer representation of shape. The track curve, a simple line, has geometric and symbolic character. Its shape roughly determines the geometry of the track. But why is it recognized as a track? Only because of the existence of the depiction of the crab, the curve gains a contextual meaning and is recognized as a track. In correlation with the vector, the curve may gain a completely new contextual meaning. The discovery of this sort of relation between elements, therefore, can result in a new interpretation of the contextual meaning of the elements themselves. The assumed process of reinterpretation could not be verified (we will see later on that there is another reasonable approach for explanation). Still, the fact that the depiction of the belt was developed directly out of the track curve by completing the loop shows that the element has changed its meaning. Figure 4-24 shows that a part of the former track (grey line on the left) has become a part the belt (grey line on the right).
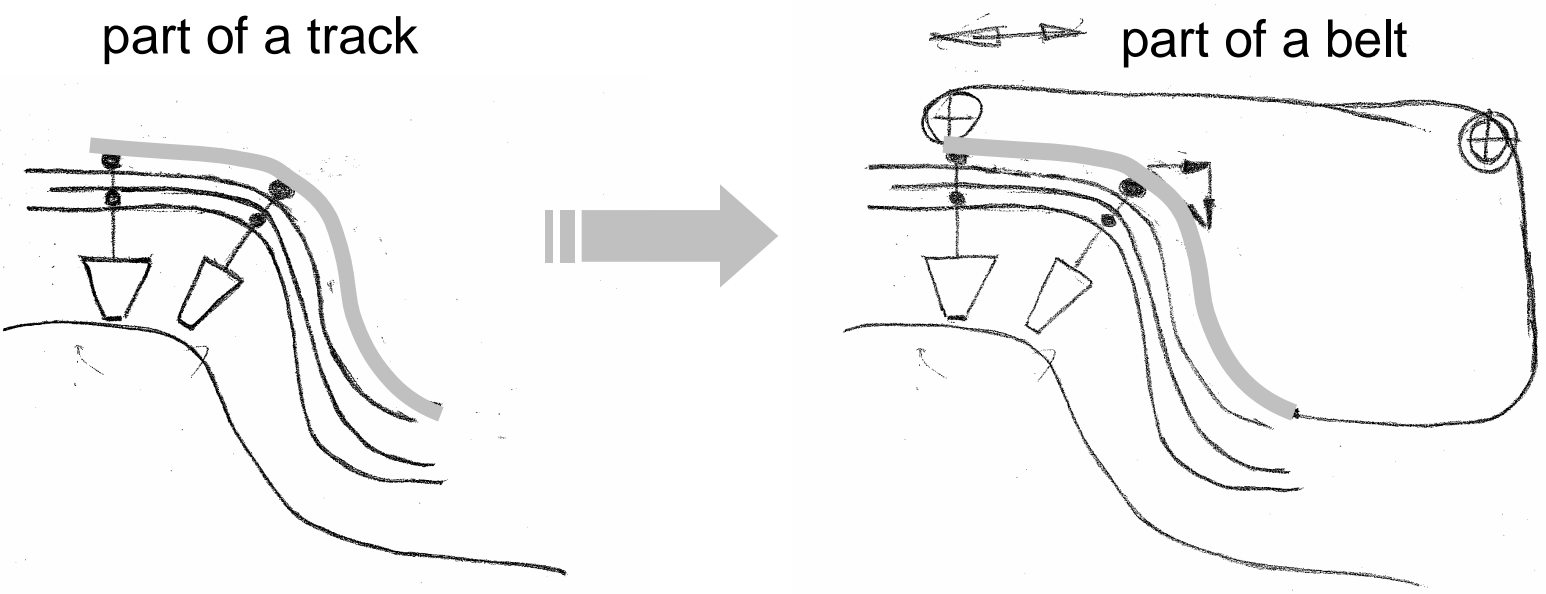

Figure 4-24: Direct development of the depiction of the track into the depiction of the belt

In this context, the sketch shows "linguistic" character. Words gain a more specific meaning when they are arranged to sentences, since then they form phrases and these phrases are correlated to each other. We have seen before that the elements in a sketch gain contextual meaning by the way they are correlated to their surrounding elements. Elements of a sketch can be considered as graphical nominal phrases, verb phrases, etc. They provide 
superordinated information when they are arranged to graphical sentences and thereby may literally tell complex technical "stories".

Throughout the whole sketching process, the actual state of the sketch is constantly visually perceived and interpreted. When elements that were depicted independently from each other are attended simultaneously, they may be correlated with each other. Since the contextual meaning of a single element depends on its relation to the other elements, this new interpretation can change an element's meaning completely. Establishing a new relation is comparable to "mixing up" the order of nouns and verbs in the technical story that is told by the sketch, so new sentences are formed. In "common" language, the syntax (which is, roughly spoken, the order of phrases within a sentence) specifies the relations between the phrases. The syntax of sketch-language is different: although the elements of a sketch are drawn sequentially, there is no one-dimensional order of elements within a sketch. Manifold directions to "read" through a sketch are possible and once the direction is changed, the new interpretation of a sketch can result in significant changes to the concept.

As mentioned before, there is another approach to explain the radical change of the conceptual solution that occurred in the design sequence described before. This approach takes into account that the track curve has been intensified before its modification to a loop. We will discuss it only briefly. When the student intensified the track curve, the motion of his hand matched the direction in which the laser was supposed to move. It is possible that the perception of the motion has been correlated to the curve, again resulting in a shape that moves in itself. This perception may not only have been visual, a kinaesthetic perception of the hand's motion is possible as well. Since the design task processed by the student is mostly a kinematic problem, this approach should be taken into account. However, such a mechanism of reinterpretation is supposed to occur less frequently since the motion is only perceptible right at the moment it takes place. It may leave a "slight trace" of remembrance, but this probably will fade soon after the event. In contrast to the perception of motion, symbols such as the arrow representing the speed vector are permanently perceptible. ARNHEIM (1977) vividly points out that especially the visual sense tends to reinterpret the perceived picture permanently. The more monotonous the picture is, the more it is reinterpreted and modified by visual perception. This effect probably even will enforce the discovery of relations between depicted elements.

In order to generalize the explanation approaches proposed above, the application of the model for soliloquy (based on SHANNON \& WEAVER 1949, see page 66) proves to be helpful. The first explanation approach, which takes visual recombination into account, is schematically represented in Figure 4-25. The sketch, considered as a communication channel, shows fundamental difference from classical communication channels (such as a telephone circuit). The information that is fed into the sketch remains in this channel. The transmission of information into the channel is a sequential process, but receiving the information is a simultaneous process. The amount of information contained in the sketch increases and due to the nature of the perception process, the designer is forced to receive the whole amount of information. Thus, information interferes and "noise" occurs (we probably use the idea of noise in a broader, less literal sense than Shannon \& Weaver suggest) and the information is altered. 


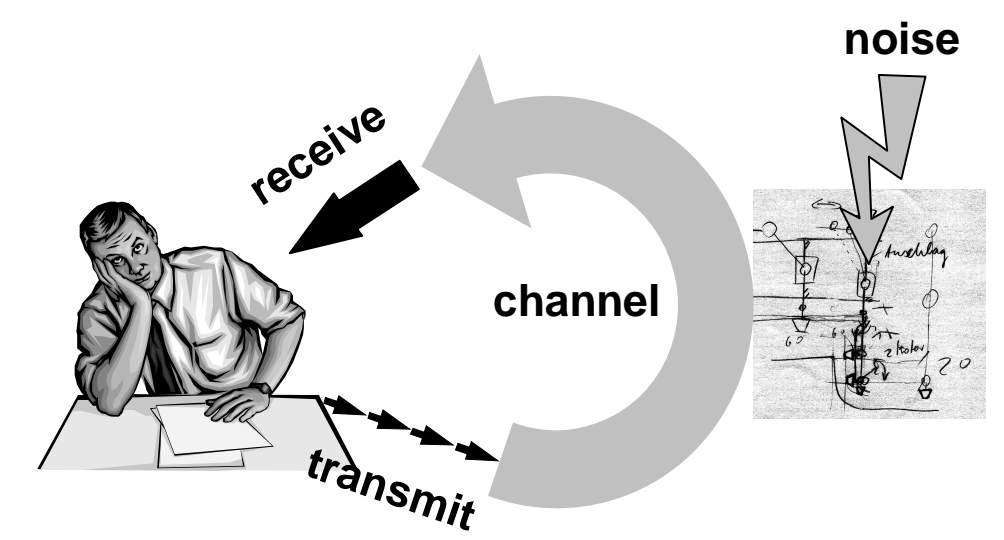

Figure 4-25: Application of the soliloquy model to the explanation approach that considers visual reinterpretation

The second approach takes the motion of the hand into account, which matches the direction of the laser's motion. The soliloquy model suggests considering the hand's motion as a message as well. The "motion information" is externalized and by means of visual and kinaesthetic action regulation it is internalized again. The medium for this communication is the hand itself, so another communication channel besides the sketch is introduced. Since the hand's motion is perceived right within the sketch, these channels may interfere with each other and the actual motion may be related to the element in the sketch (although the element actually does not move). Figure 4-26 schematically shows the application of the soliloquy model to this case.

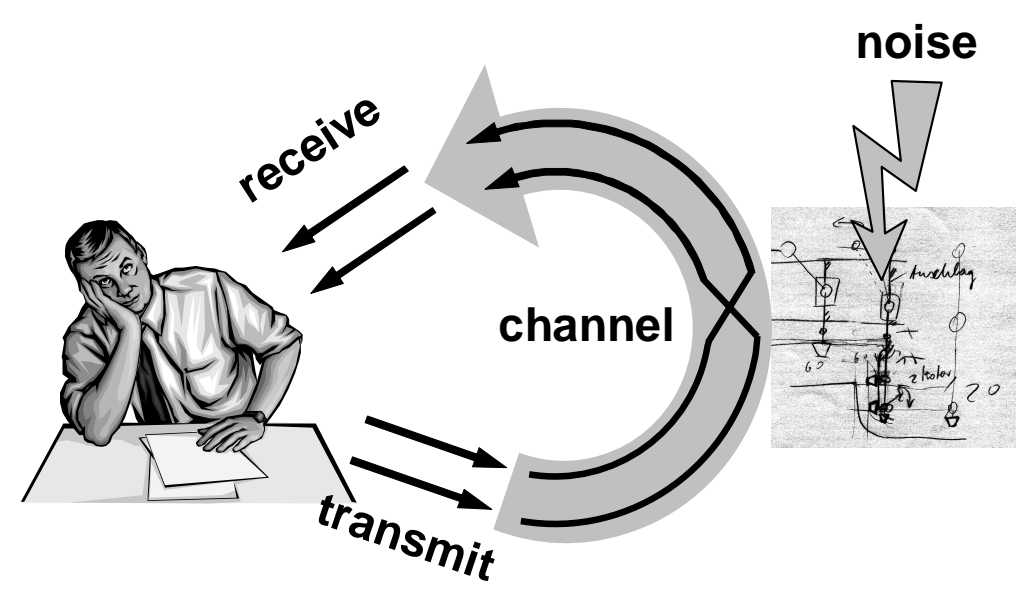

Figure 4-26: Application of the soliloquy model to the solution approach that considers the simultaneous perception of the sketch and the hand's motion 
A major advantage of the soliloquy model is that it thoroughly differentiates between the processes of externalization and the processes of internalization. Furthermore, it takes into account that the message is altered (by noise) while it is "transferred" by the sketch, although the pieces of information themselves do not change. SHANNON \& WEAVER (1949, p. 12) define different levels of communication problems and the alteration of the message that we discussed above is a semantic alteration - the meaning of the message is "misunderstood" ("level B" communication problem - see page 65). The soliloquy model allows for the integration of processes of "pure" action into an information processing model. Thus, this model combines action theory and problem solving theory for the study of design processes.

The example above shows that in creative processes, action and cognition may be interlocked closely indeed. Let us regard the first explanation approach: the sequential character of the action "depict shapes on a piece of paper" in combination with the simultaneous character of visual perception provides a new combination of depicted elements. Due to the linguistic character of such elements, the new combination expresses a completely new "statement." By cognition, a technical component is found, which matches this new statement. Such a process may bring about a completely new conceptual solution - according to classical scientific frameworks, such a result would probably be assigned only to processes of pure cognition. In this case, action, perception and cognition work hand in hand - and cognition probably plays the least important role among those three.

Unfortunately, the mechanisms proposed before could not be verified in this experimental setting. The student was not told to "think aloud", therefore design events can only be identified by observation of the sketching process. Unequivocal conceptual changes have occurred rarely and they mostly eluded from well-founded interpretation. Still, the proposed mechanisms do not contradict the findings from other design domains. Due to the domainspecific modality of sketching in mechanical engineering design, the occurrence of such mechanisms may have an impact on the solution development process that is different from the impact within other design domains (see GERO \& TVERSKY 1999 for examples from architecture). In mechanical engineering, reinterpretations on a "rather simple" geometrical level may have a major impact on the underlying contextual meaning of the sketch and, thereupon, may result in new conceptual solutions.

\subsection{The Meaning of the Pencil's Motion in Sketching}

Apart from the processes of cognition and perception that are underlying and accompanying the sketching process, the depiction of lines with the help of a pencil is action. It requires the application and regulation of physical, muscular work in order to achieve the motion of the pencil's tip. As long as the tip touches the piece of paper thereby, a line appears that is shaped in accordance with the direction of the motion. Not without reason, we have chosen the depiction of a square to exemplify the TOTE-cycle as a basic model of action regulation (MILLER et al. 1960) in chapter 15. As we have seen before, the depiction of a line may be a part of regulation cycles on a high level of complexity, such as the representation of a shape. The regulation cycle may again be part of a cycle on an even higher level, such as the representation of a complex technical system (see page 16). A hierarchical structure arises 
that describes the execution of complex tasks. Still, on the bottom level of this structure, there is a sequence of observable actions (HACKER 1998, pp. 203).

Besides sketches, there is another, very basic representational medium that allows the "depiction" of lines and shapes but does not require any action, which is imagery. BADDELEY (1997, pp. 71) proposes a working memory model that includes a system for the mental manipulation of images and, revealingly enough, calls this system the visuo-spatial sketchpad. According to the vision system by KossLYN (1994, pp. 70), mental images may be generated from memory without an actual stimulus from perception. Likewise the mental image of a sketch may be generated and manipulated on the mental sketchpad. Still, the vision system may as well generate a mental image from actual perception and then process it, so an existent sketch may be viewed, stored and then manipulated in mind.

From theoretical consideration, three different ways to generate, manipulate and process any sketch-like information may be proposed (see Figure 4-27). First of all, a designer may draw "real" lines within an actual sketch. These depictions are perceptible and lasting. Secondly, he may watch an actual sketch and thereby imagine its alteration. Thereby, a mental image is generated from perception which is manipulated then. The existent part of this mental sketch is refreshed constantly, but the manipulated part is non-durable. Lastly, the designer may generate and manipulate a mental sketch without any actual perception. This may happen while he closes his eyes, watches an empty piece of paper or stares into space. Such a mental sketch is entirely exposed to the process of fading, blurring and forgetting that is characteristic for imagery (KOSSLYN 1994, pp. 74).

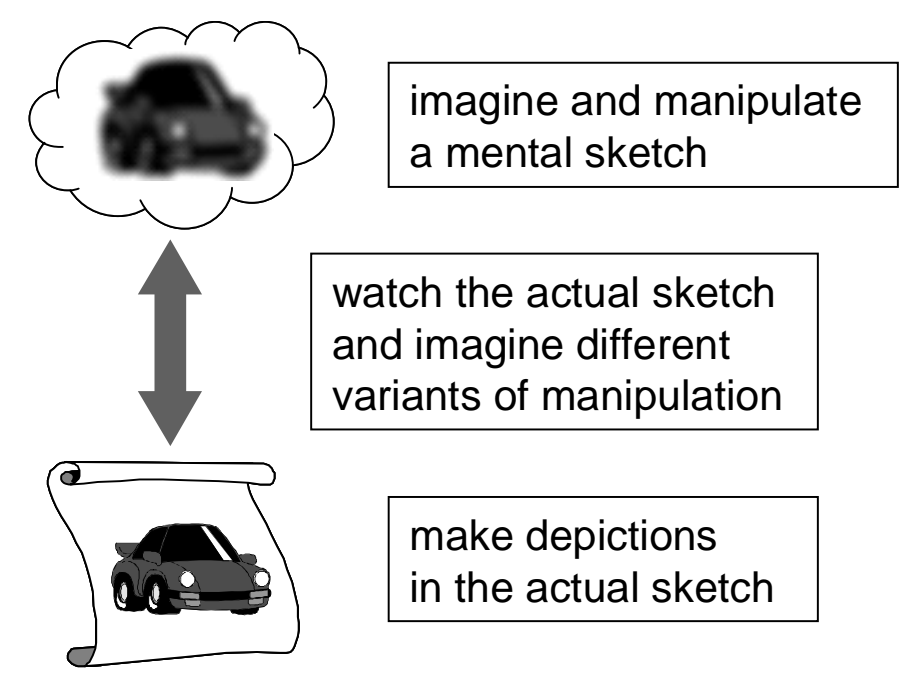

Figure 4-27: Three levels of depiction for sketch-like information

Going back to statistical analyses on the general run of a sketching process (chapter 4.2), we see that watching the sketch holds a considerable share of conceptual design activities. Those test subjects that were considered as corner cases watched the sketch for $26 \%$ and $44 \%$ of the overall time respectively. We must admit that watching a sketch does not necessarily 
imply that it is mentally manipulated at the same time. The sketch may be regarded for purpose of analysis as well. However, there is a considerable period of time when mental manipulation could happen. By contrast, the corner case designers stared into space fairly seldom $\left(1 \%\right.$ and $3 \%$ respectively $\left.^{1}\right)$. Likewise, this activity may not be equated with processing a mental image, since verbal reasoning may take place during this time as well. Under the experimental conditions used in this study (no request to "think aloud"), the processing of mental images could not be analyzed directly, anyhow.

In order to find an approach to depiction and manipulation of sketch-like information that goes beyond actual lines on a piece of paper, we will view another case study. Again, video data from the experiments was used to investigate all observable actions that are carried out during the sketching process in great detail.

For that reason, the behavior of the designer with the highest rate of sketching activity per time is investigated (test subject no. 34, which has been regarded as a corner case in chapter 4.2). The following design sequence is taken from the barbecue grill experiment. The analysis starts immediately after problem clarification when the first element has been depicted that has not been given in the task description. It ends when all elements necessary for the function of the grill have been developed and thereby, the first conceptual solution approach is completed. All in all, this phase just lasts some minutes.

The following categories of action are distinguished by the protocol analysis ${ }^{2}$ :

- pointing at a free spot

(touching the paper's surface with the pencil tip or bring the tip near to the surface at a spot where no depiction or no characteristic point of a depiction ${ }^{3}$ is - remaining motionless at this spot for a noticeable while - lifting up the tip again without having depicted anything)

- pointing at a given element

(same procedure as pointing at a free spot, but pointing at a characteristic point of a given depiction)

\footnotetext{
${ }^{1}$ These figures even cover the recording of activities that could not be assigned to any other protocol category.

${ }^{2}$ These categories were developed by explorative spot checks that were carried out randomly among all video data. They fairly follow the physical actions proposed by SuWA et al. (1998, pp. 460), which are distinguished in drawing actions (D), moves (M) and looking at depictions (L). In this case, L-actions were not noticed due to the experimental setting. The categories used in this analysis may be regarded as differentiations and integrations of the sub-categories of M- and D-actions by Suwa et al.

${ }^{3}$ End-points, corner-points or centre-points of depictions were regarded as characteristic points. Points that were indistinctly located within a depiction were regarded as "free spots" as well.
} 
- gesture the depiction of an element ${ }^{1}$

(bringing the pencil tip near to the paper's surface without actually touching it - moving the pencil in a drawing manner without actually making any depiction)

- actually depict a new element

(touch the paper's surface with the pencil tip - actually draw a line that has not been depicted before at this position)

- actually re-depict a given element

(same as actually depict a new element, but thereby follow the shape of a line that was already depicted right at this position)

- actually write down information

(depict a word, a sentence or a paragraph)

The resulting protocol features those single actions that cannot be subdivided any further (according to the description above) as the smallest entity and scale unit as well. The time elapsed was taken down roughly, but the actions were not correlated with time, so the duration of actions did not matter for the protocol ${ }^{1}$. Figure 4-28 shows the protocol that emerged from observing the actions of test subject no. 34. A black mark indicates an action that refers to empty spots and new elements in the sketch; a grey mark indicates actions that refer to given elements and their characteristic points respectively. The test subject worked on three sketches as indicated in the protocol.

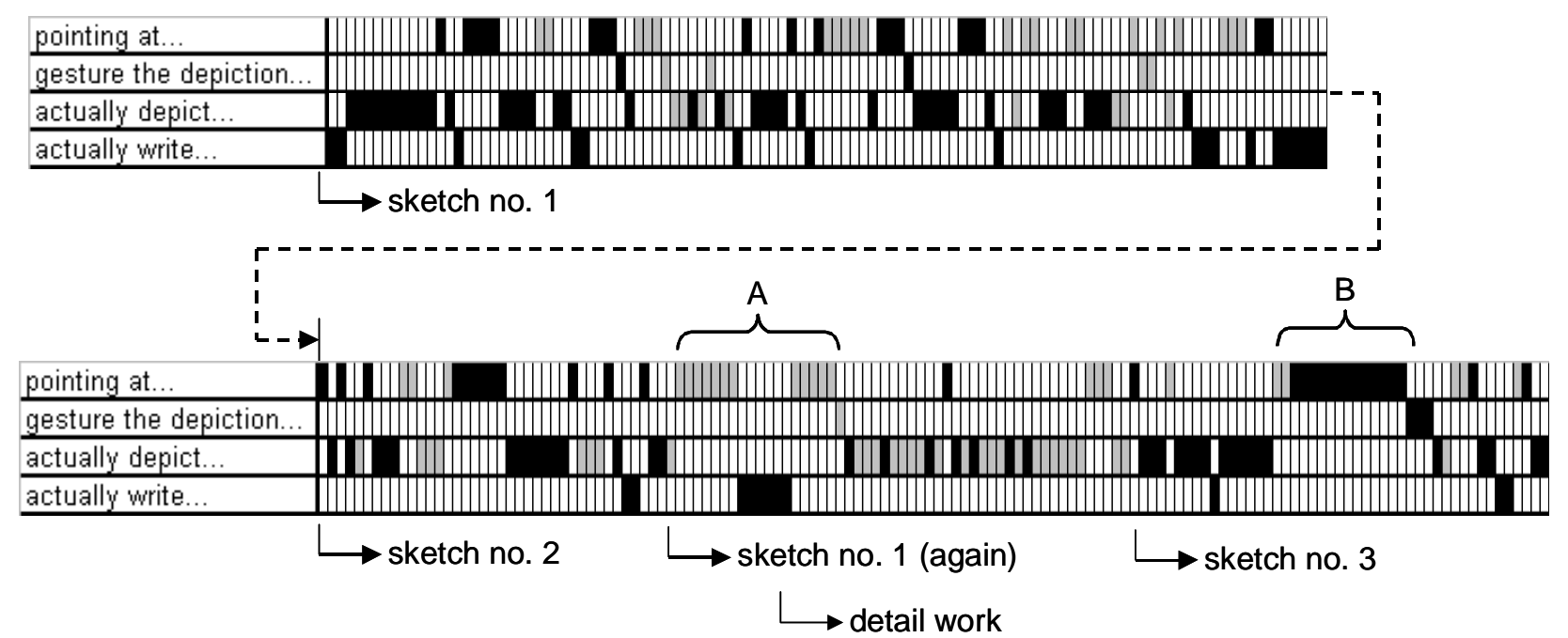

Figure 4-28: Protocol of actions for test subject no. 34

\footnotetext{
${ }^{1}$ In the protocol, gesturing the depiction of a new and a given element was distinguished, but for most analyses made in this study, both these categories were summed up.
} 
When the frequency of the occurrence of certain action categories is referred to the total number of actions, an interesting picture emerges. Figure 4-29 presents the distribution of action categories for the whole duration of observation. It shows that the depiction of new elements $(32 \%)$ is the action category that occurs most frequently. Summing up all kinds of depiction action (new elements and given elements), depiction forms about the half of all actions (48\%). Still, a number of other categories hold a distinct share in the overall process as well. Pointing in the sketch with the tip of the pencil takes up more than a third of all actions (38\%), while this action is rather evenly distributed among pointing at free spots $(20 \%)$ and pointing at the endpoints of given elements $(18 \%)$.

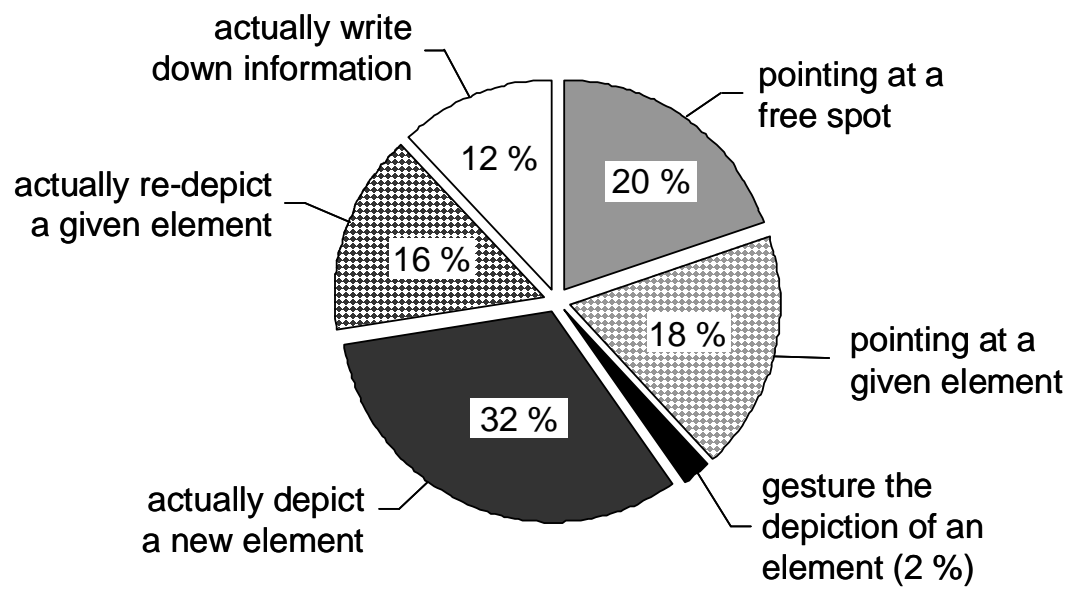

Figure 4-29: Distribution of action categories for test subject no. 34

In other words, one might state that "the process of sketching does not consist of sketching exclusively." Pointing at elements and spots is an active occupation with the sketch taking place within the sketching process. Going back to the overall action protocol, it seems that the process of pointing may partially be seen as a sub-process that can fairly be differentiated from depiction. Sequence A (see Figure 4-28) shows a sub-process that seems to be secluded from the overall process and mainly consists of pointing at given elements. Furthermore, sequence B shows a similar sub-process that consists of pointing at free spots in the sketch, though. Later on, we will further analyze both these sequences. Apart from both these sequences, the action of pointing is evenly distributed among the rest of the process and seems to be an integrative part of depiction, too.

In order to further investigate the contextual meaning of certain actions and sequences of action respectively, each action was related to its object, which is the respective element or spot in the sketch. Figure 4-30 exemplifies the detailed protocol that covers the designer's first occupation with sketch no. 1, in which he developed the basic configuration of the

\footnotetext{
${ }^{1}$ According to the subjective impression of the observing person, the duration of actions ranges from about ten seconds (writing down words) down to the tenth of a second (pointing at spots).
} 
barbecue. Figure 4-31 shows the sketch at the end of this sequence and a schematic representation of the sketch. This schematic representation illustrates the order of depiction of lines (small letters) and pointing at spots (capital letters). The actions are indicated by the same letters in the protocol (Figure 4-30) as well. The solution consists in a three-legged rack $^{1}$, a grate that is hung up on the rack and a coal tub that is placed underneath the grate. The cable or chain that holds the grate is guided over a coil underneath the top of the rack and is wound up around a winch that is attached to one pole of the rack.

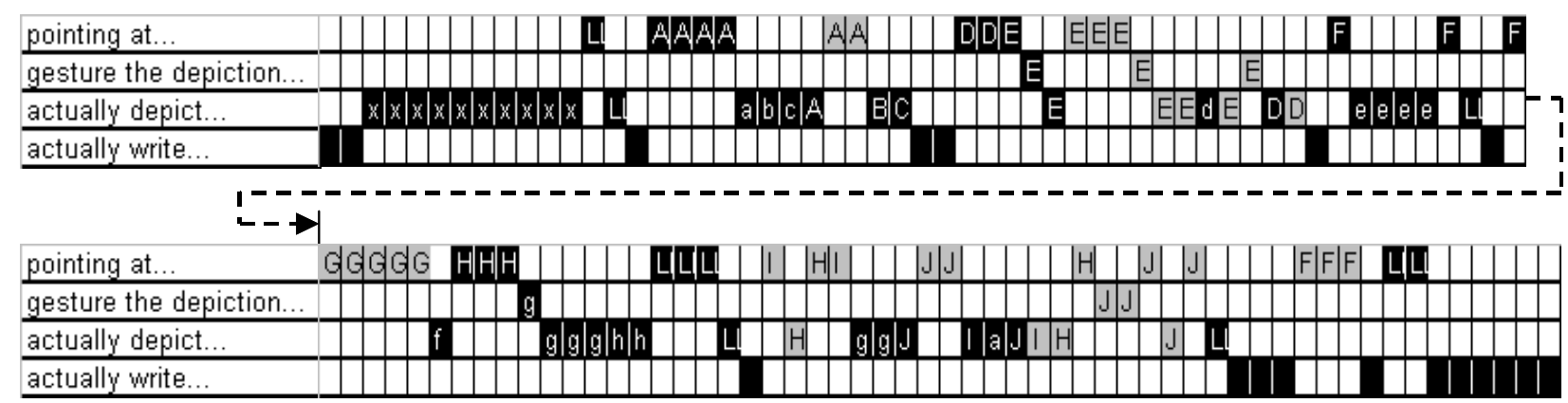

Figure 4-30: Detailed protocol of actions for the designer's occupation with sketch no. 1

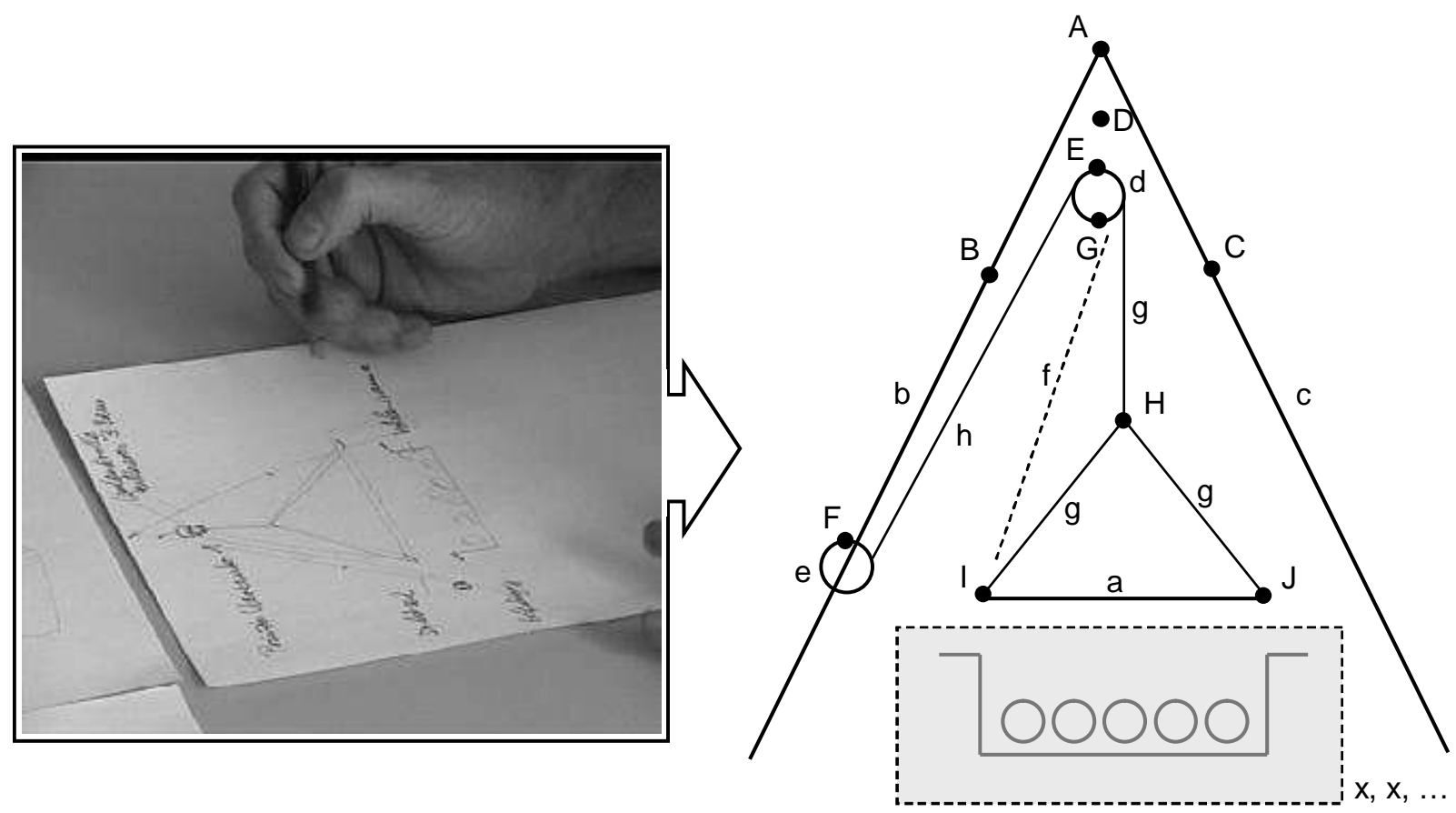

Figure 4-31: Schematic representation of sketch no. 1

\footnotetext{
${ }^{1}$ Only two legs are depicted due to 2-dimensional representation.

This book is available at Verlag Dr. Hut, München, www.dr.hut-verlag.de (ISBN 3-89963-176-5)
} 
From those action categories that occur rather frequently, depiction is probably the category that is most ordinary for a sketching process. Still, pointing at elements or spots in the sketch, using the pencil's tip as a pointer, seems to be a distinct part of the sketching process. Both sequences show pointing action almost exclusively (A and B in Figure 4-28) and they allow for assumptions on the meaning of pointing.

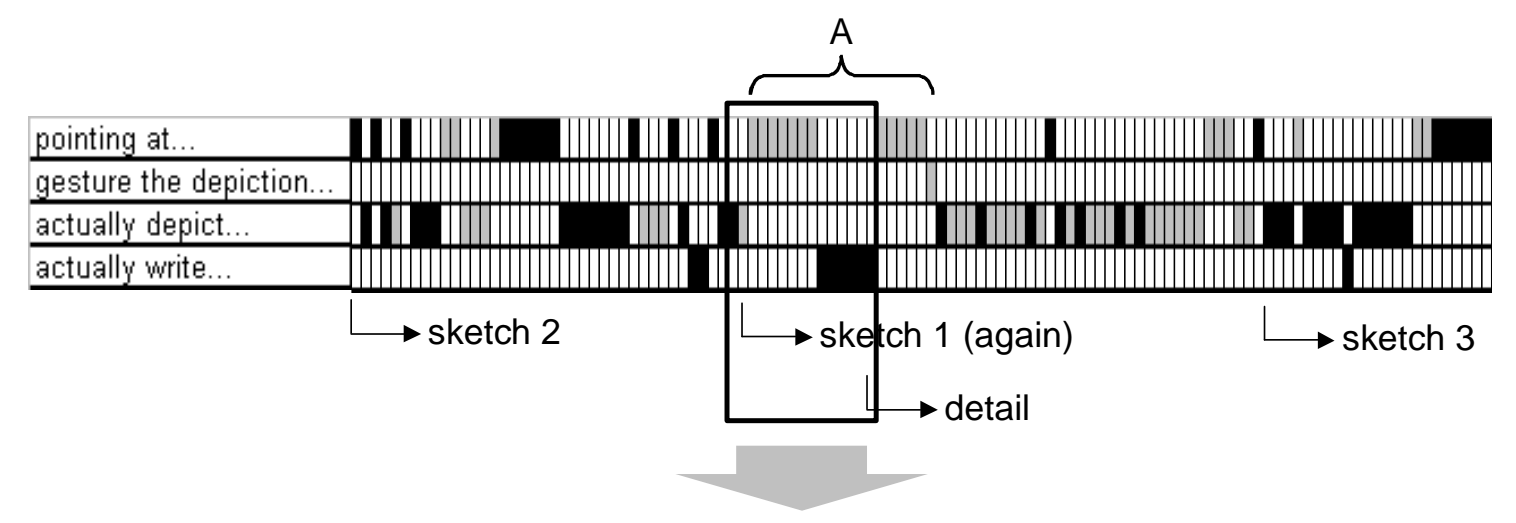

pointing at...

\begin{tabular}{l}
\hline gesture the depiction... \\
\hline actually depict... \\
\hline actually write... \\
\hline
\end{tabular}

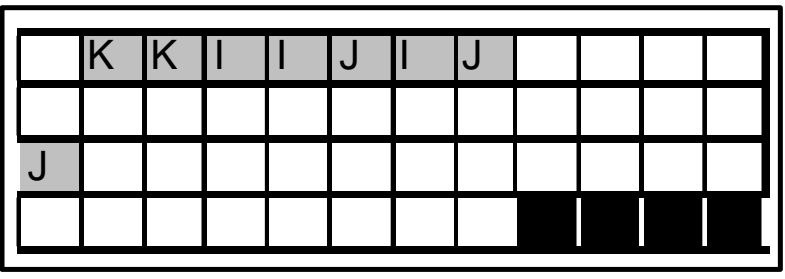

Figure 4-32: Detailed protocol of actions in sequence A

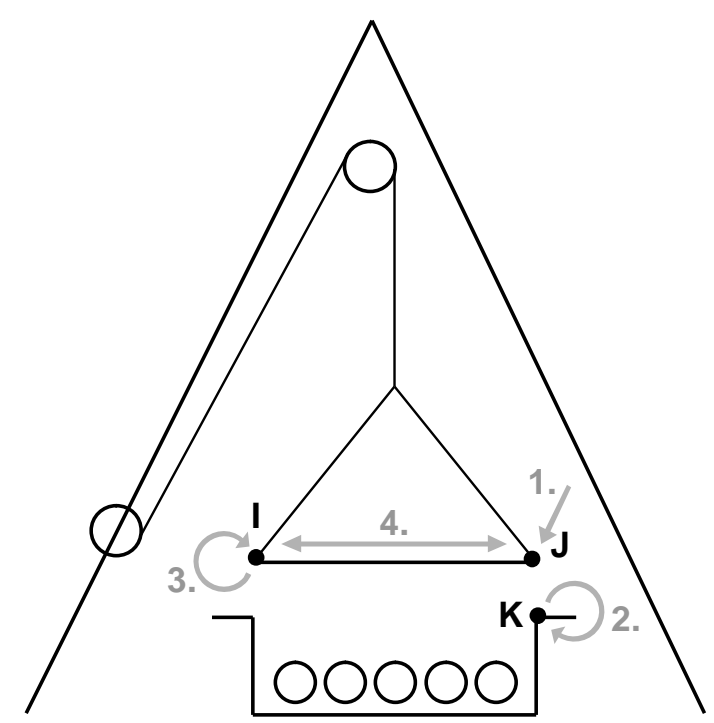

Figure 4-33: Pointing action in sequence A

This book is available at Verlag Dr. Hut, München, www.dr.hut-verlag.de (ISBN 3-89963-176-5) 
In sequence A, the tip of the pencil wanders across elements that have been depicted already, as shown in the protocol of this sequence in Figure 4-32. Immediately before, the designer has worked on the second sketch, in which he has specified the winch for winding up the cable or chain. Now he returns to the sketch he has drawn at first (see Figure 4-33) and redraws the endpoint $(\mathrm{J})$ of the grate. Afterwards he points twice at the corner point $(\mathrm{K})$ of the coal tub, then points twice at the other end (I) of the grate and finally switches between pointing at both ends of the grate (I and J).

We can only speculate on the meaning of this sequence and on its underlying cognitive process. But it may be supposed that this series of pointing action was not part of an intended depiction process. On a conceptual level, the solution is complete at this point and the sketch has not been altered, supplemented or extended in this area then or later. Before this sequence, the designer has been engaged with a different sketch and a different aspect of the solution. Immediately afterwards, he writes down information according to the way in which the poles of the rack may be put together ${ }^{1}$. Contextually, the sequence is isolated from the preceding as well as from the subsequent action sequence and it does not relate to any depiction made in this sketch afterwards. Accordingly, it may be supposed that the series of pointing action was not part of synthesis via depiction, but served to analyze, assess or check the actual solution. The pencil tip may be used as a marker to guide the eye through the sketch. When the pencil tip points at a certain spot in the sketch, it increases the contrast of that spot to the area that surrounds it. The "emphasized" element stands out against the other elements and therefore the eye does not stray. Due to the experimental setting, there is no way to consider what kind of analysis, assessment or verification has been carried out by the designer. Since the designer is engaged with the grate and with the upper corner of the coal tub alternately, we may suppose that the designer deals with some relation between both these solution elements. Such a relation may be the proportions of the parts or the relative motion of the parts according to each other. Yet there is another interesting sequence in which the designer is occupied with the grate and the coal tub, which is sequence B. The proposed process of analysis in sequence A may have been the preparation for this sequence.

\footnotetext{
${ }^{1}$ After the pointing sequence, the designer writes down "Stangen wie Zeltstangen zusammenstecken", which means in English "poles are fit into each other like tent poles".
} 
Sequence B (see Figure 4-34) also mainly consists of pointing actions, but in this case the designer points at empty spots and at non-characteristic points of elements respectively. Again, it can only be speculated on the underlying cognitive process. Directly before this sequence, the designer has drawn the coal tub on a new piece of paper in the same configuration he already depicted it in a previous sketch. Afterwards (as shown in Figure 4-35), the designer points at indistinct points on both side walls of the tub (A and B). This possibly happens in order to judge the distance between the walls or to imagine a future element that may be located between those walls. Then a remarkable part of this sequence follows. The designer points eight times in a row at a spot above the coal tub (C) where there is no actual depiction (yet). Afterwards, he points twice at a point near the tub's side wall (D). He then moves the pencil tip back to point $\mathrm{C}$ and without actually touching the paper's surface. At this level, he "twitches" several times (just above the spot where the latter depiction of the grate will be located), just as if he would start to draw a line. Directly afterwards, he finally lowers the pencil down to the paper's surface and finally draws a line that represents the grate above the tub. What may have happened within the few seconds described above?
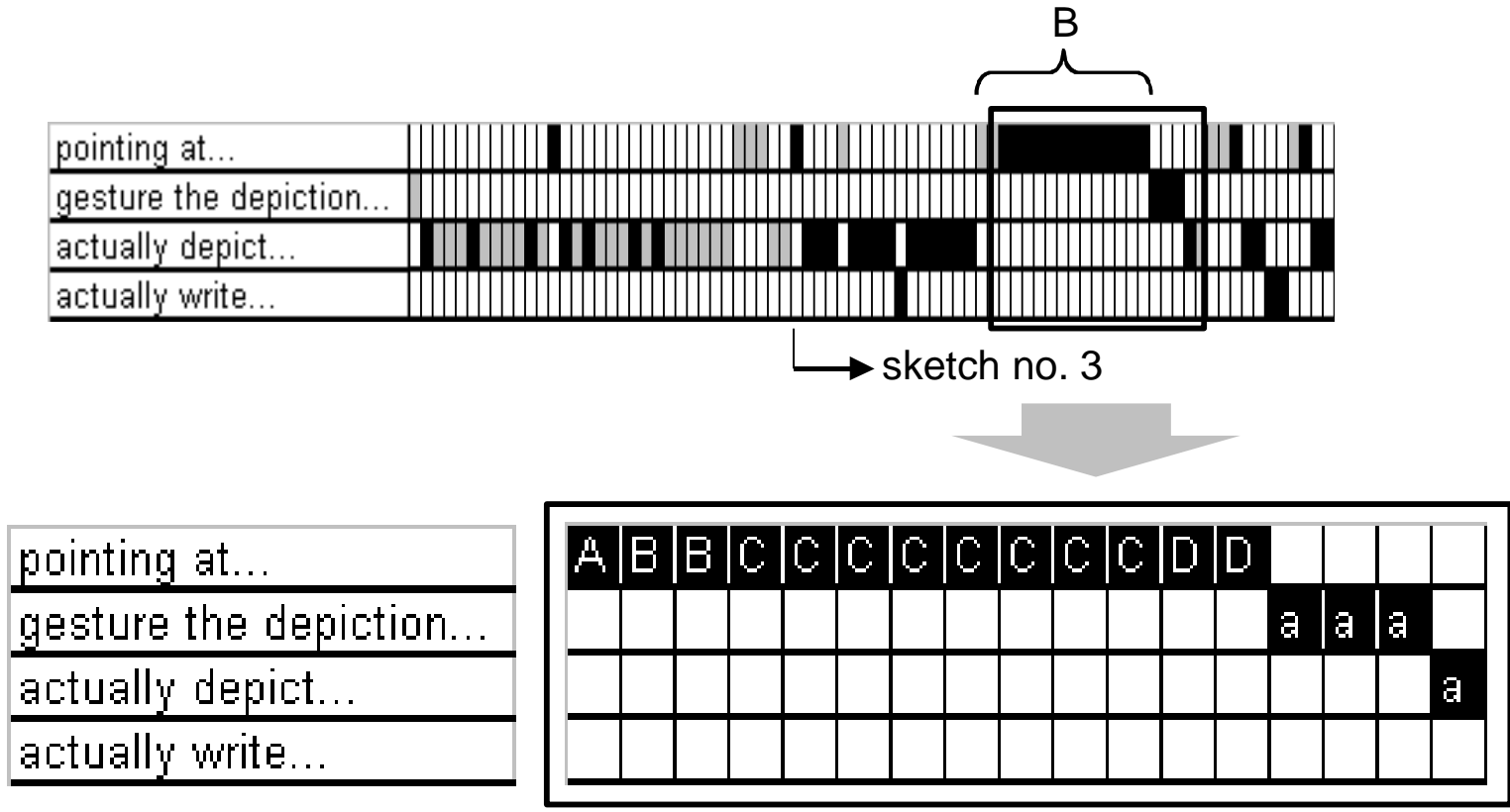

Figure 4-34: Detailed protocol of actions in sequence B 


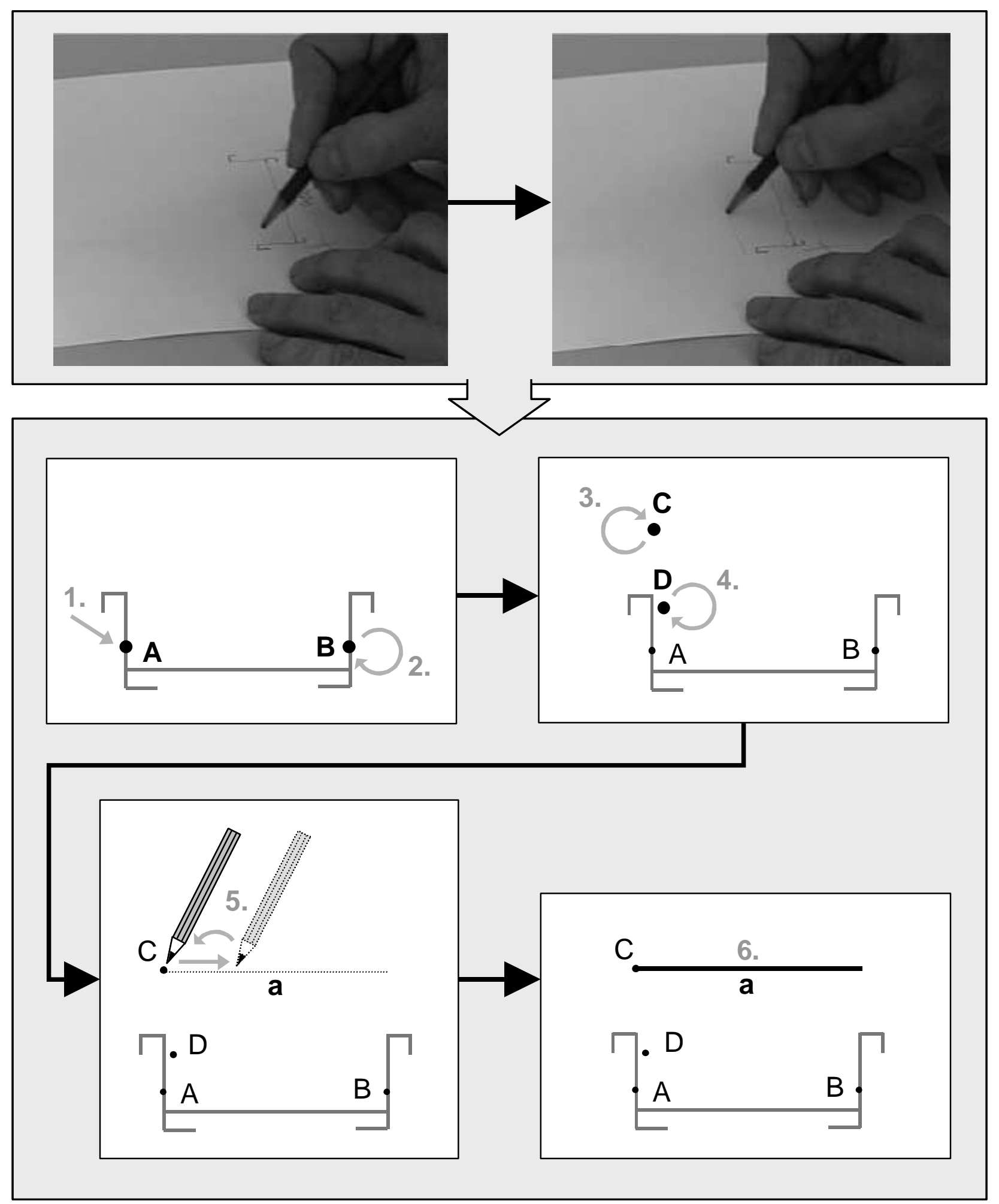

Figure 4-35: Pointing action and gesturing in sequence B 
The designer points at $\mathrm{C}$ at a moment when there is no depiction yet. Still, this spot gains meaning after this event, since at this spot there will be the depiction of the grate. Obviously, the designer assigned relevance to this spot even before the depiction, since he pointed right at this spot. When he pointed at $\mathrm{C}$, he probably thought ahead and already imagined the grate at this spot. We already discussed that pointing at a depiction with the pencil tip increases the depiction's contrast to its surrounding elements. This applies to empty spots to an even greater degree, since without the pencil tip there is no contrast at all. It is hard to fixate a particular empty spot on a piece of paper when there is no visual clue to hold on to. This is even more difficult, when there are contrasting elements in the surrounding space ${ }^{1}$. The reader himself may try to fixate a random empty spot on a sketch for some time without straying - this sounds like a simple exercise, but in fact it is rather hard to do!

In this case, the assumption is that the designer imagines the depiction of the grate being within the sketch while fixating the empty spot with the help of the pencil tip.

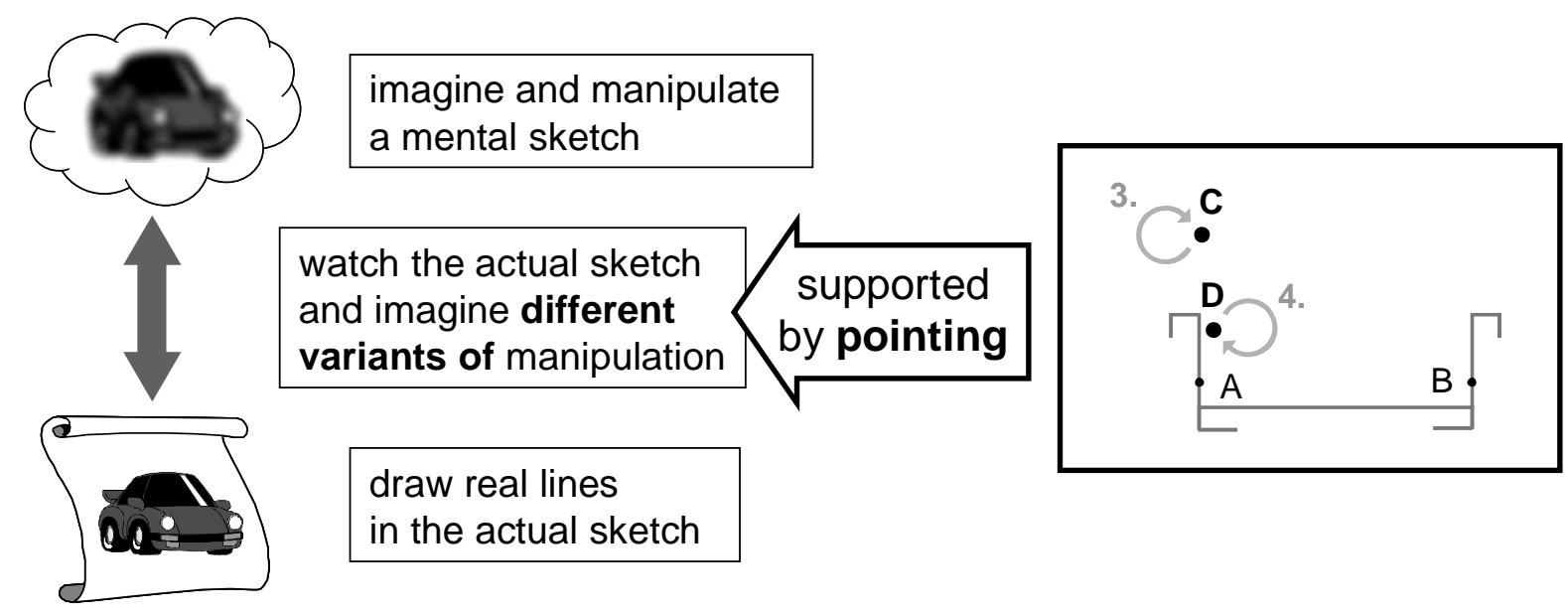

Figure 4-36: Three levels of depiction for sketch-like information

Going back to the theoretical levels of processing sketch-like information (they are shown in Figure 4-36 in an adapted way), the level of "watching the sketch and imagining its alteration" takes on a wider complexity then. It seems to be supported or even complemented by the process of pointing on spots and elements within the sketch. Moreover, this level actually seems to be used for the generation of different solution variants for a design problem. The designer points at spot $\mathrm{C}$ (8 times), points at $\mathrm{D}$ (twice) and then depicts the line to represent the grate at the location of $\mathrm{C}$. So why does he point at $\mathrm{D}$, which - at first glance seems to be an interruption of his process of imagination? It may be supposed that the designer tested several different positions of the grate. One variant would be on the level of

\footnotetext{
${ }^{1}$ Arnheim (1969, pp. 23) vividly describes the mechanism of fixation that urges the eye to bring outstanding objects into the centre of the field of vision. This reaction is determined to a greater extent by the stimulus than by the person's conscious intention.
} 
point $\mathrm{D}$, right between the side walls of the coal tub. This position would have the advantage that the hanging grate could not swing during grilling, so the meat could not fall into the embers. However, the grate could get stuck during the adjustment of its height and then abruptly bounce up, so the operating person might even get hurt. The designer might have carried out such a consideration during this sequence and then decided to dismiss a grate that hangs between the coal tub's walls. Instead he chose to locate the grate at $\mathrm{C}$ above the tub Figure 4-37 shows both solution variants, which clearly differ from a conceptual point of view. The level of "watching the sketch and imagining its alteration" is possibly not only used to "pre-think" a following depiction in order to (re)call the sequence of actions. It may rather be used for the cognitive process of generation and evaluation of different solution variants and decision for one of those variants ${ }^{1}$.

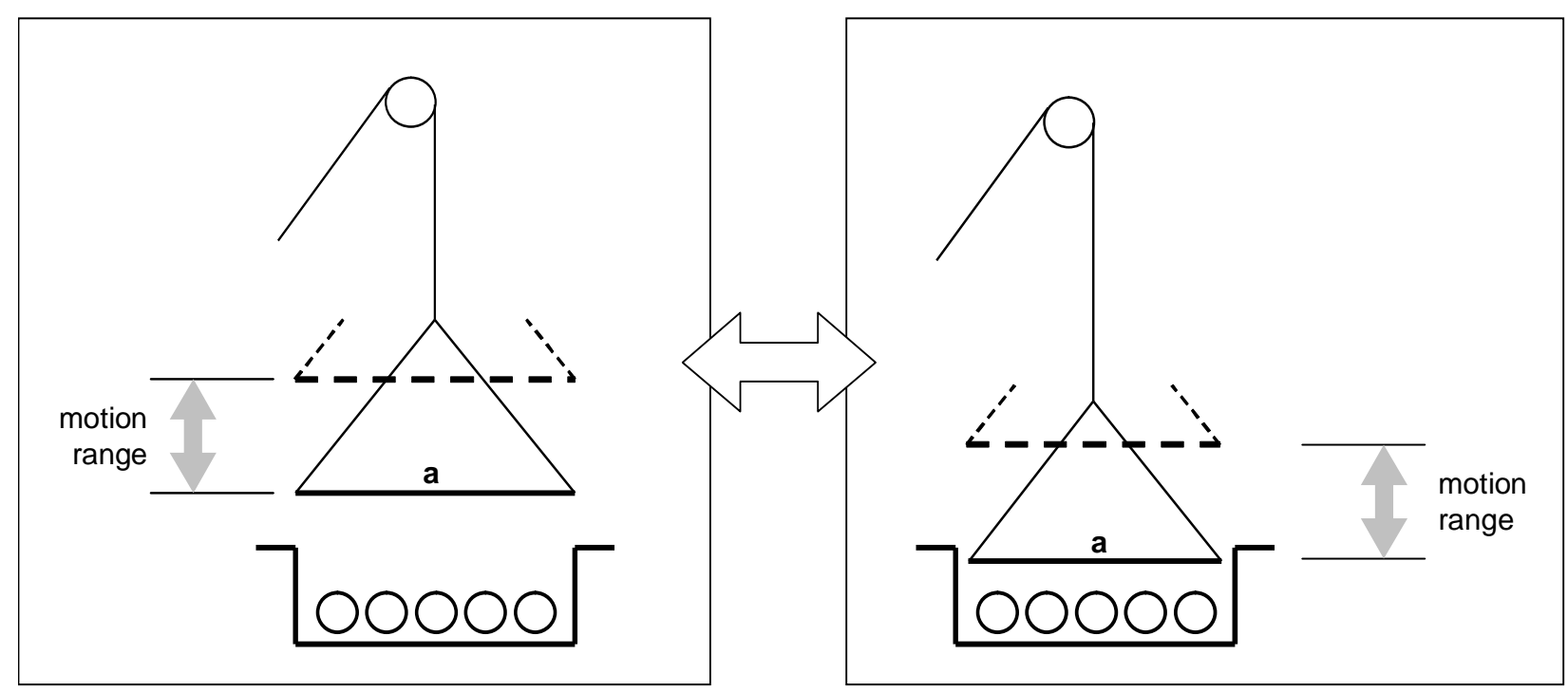

Figure 4-37: Both solution variants for the hanging grate

Still, one interesting detail of sequence B remains to be discussed. Near the end of the sequence described above, the designer twitches with the pencil's tip as if he would draw a line. But since the tip does not touch the paper, no real line emerges. Still, the motion is just where the actual line will be depicted subsequently (see Figure 4-35 once again to recapitulate this scene). Alleging that the designer does not twitch for non-contextual reasons (such as nervousness), we may suppose that the designer "imitates" the real depiction by this gesture. The gesture may be a trial-action without a permanent consequence - it is a "test drive" for the real depiction that follows then. While gesturing, the designer performs an action that is basically similar to the real depiction. Hence, he receives a similar sensory-motor feedback from the gesture. Moreover, the designer receives a visual clue to the future depiction,

\footnotetext{
${ }^{1}$ Generation and evaluation of and decision for solution variants is reminiscent of distinct stages of the problem solving cycle suggested by Ehrlenspiel (2003, p. 81).
} 
although it does not exist yet. The designer can see the direction of the pencils motion which matches the future line. Kim \& Francis (1998) argue that when moving objects are watched, there is a sensual stimulus of the motion line ${ }^{1}$ (somewhat comparable to the lines in the comic picture shown in Figure $4-38^{2}$ ). This sensual impression is not lasting though - it fades away after a short time. So the designer's gesture may generate a perceptible representation of the line and the shape of the depicted element, respectively. Yet, this representation vanishes after a while - in a figurative sense, the line erases itself.

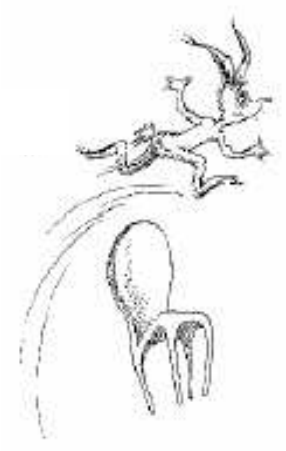

Figure 4-38: Comic picture showing motion lines

(reprinted from Seuss, 1968 by Kim \& Francis, 1998)

On condition that the twitching of the pencil is applied in the manner that has been proposed before, such a behavior would create a new level of "depiction." Compared to the levels described before, gestures differ substantially from the other kinds of depictions as shown in Figure 4-39. Just as real lines, gestures provide the representation of an element in a perceptible way. Yet, the perception of the gesture is not permanent - it is momentary. Hence the depiction is not lasting, but fades and blurs just as the imagined manipulation of an actual sketch. In contrast to pointing action, gestures make the shape of the depiction itself perceptible.

\footnotetext{
${ }^{1}$ Kim \& Francis (1998) show this effect theoretically by computer simulation of a neural network. The network model generates trails of different orientation depending on the speed and the length of the motion.

${ }^{2}$ Friedmann and Stevenson (1975) found that motion lines influenced childrens' judgements of movement in still pictures. Motion lines can even be found in $11^{\text {th }}$ century Japanese drawings ((Friedmann \& Stevenson 1980).
} 


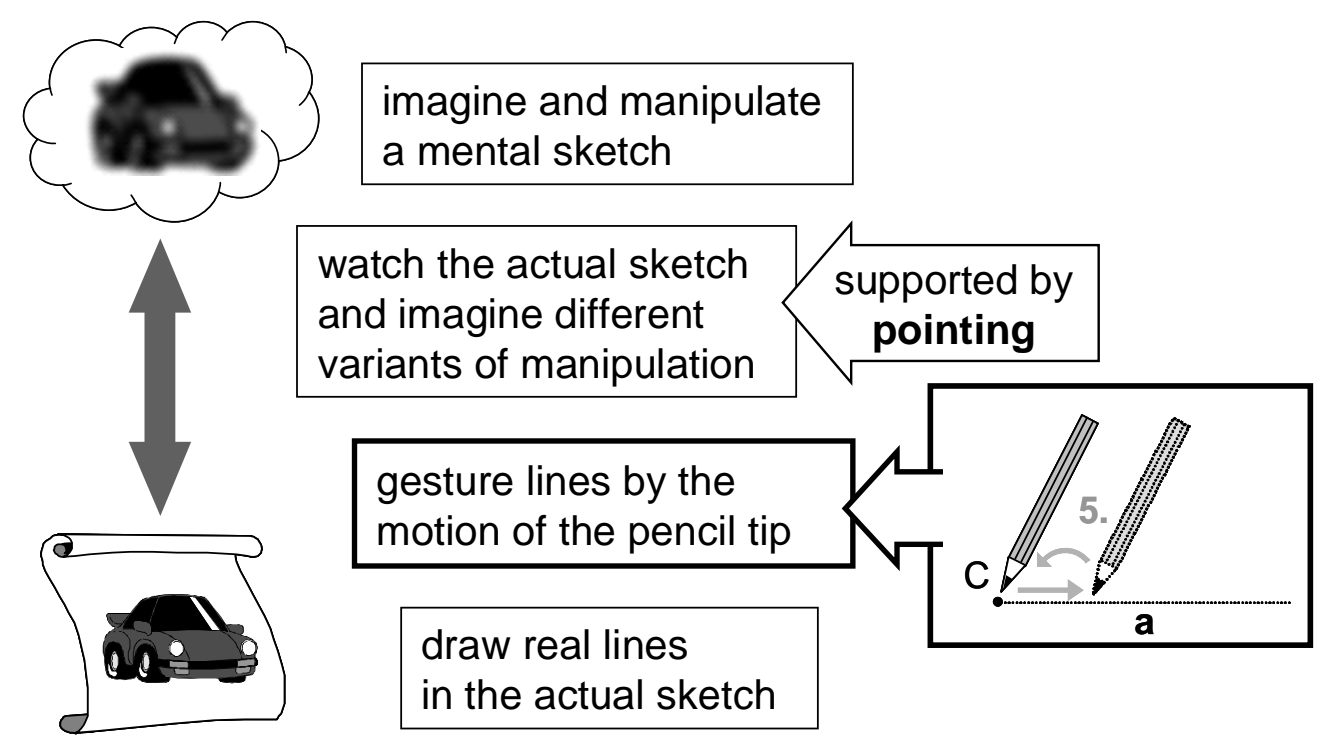

Figure 4-39: Four levels of depiction for sketch-like information

From the observations described above, it may not be safe to conclude that designers perceive gestures as representations of potential future depiction. Furthermore, we do not know if designers use gestures in a cognitive way, for example in order to analyze the behavior of the object that is represented by the gesture. Although gestures have occurred less often than pointing actions and "real" depictions, this matter should be investigated with the help of an appropriate experimental design. Due to the chance of providing a unique level of depiction, it should be considered if the use of gestures as depictions may be taught in design education, even if gestures do not prove to be relevant in designer's "natural sketching."

From sheer consideration of the frequency of occurrence, pointing seems to be an integral part of a design sketching process just as drawing lines itself. It suggests the relevance of a level of depiction that combines the perception of the existent sketch with elements in imagination. Gestures may even expand the bandwidth of levels of depiction by a perceptible line that erases itself after a while (unless it is actually drawn afterwards!). A fluent change between those levels is possible, since no change of medium or tool is necessary. These levels have in common that they are all active occupation with the sketch and within the sketch. 


\subsection{A General View on the Sketching Process}

In the preceding chapters, we have considered sketching as a process that comprises...

- problem solving,

- creativity,

- action regulation,

- perception,

- communication and

- linguistic aspects.

Each one of these characteristics makes contributions to the development of conceptual design solutions by means of sketching. Thus, conceptual design sketching shall be seen as a highly complex process that combines a number of mechanisms that interact with each other.

First of all, sketching offers the possibility to externalize provisional and partial results of a mental design process. By using sketches as intermediate information storage, the designer can overcome limitations of the working memory in order to handle complex technical systems. The designer may use specific geometric depictions to show the shape of objects and their geometrical interrelations. Likewise, he may use words and symbols that are conceptually abstract in order to represent a variety of objects at once. Thus, concrete and abstract information may be externalized in sketches to support a problem solving process that is based on switching between different levels of abstraction (DÖRNER 1999A). Moreover, concrete and abstract predefinitions may be combined in one single sketch. Geometrical relations between parts of a solution may be represented in this way, even when those parts differ in their level of concretion and completion respectively (see Figure 4-14 on page 87).

Elements of a sketch may also be abstract with reference to their shape; we have labeled such elements schematic. In chapter 4.4, we have considered a sketch that features a curved line to represent a track in which a crab runs (see Figure 4-21 on page 96). This element provides geometrical information about the track, since its curvature shows the direction of the track in the tangential dimension. Still, the curve leaves open the extension in the other dimensions. So the depiction contains certain geometrical information and at the same time, it omits other information that would be necessary in order to entirely specify the represented object. Thereby, this line may be used as an abstract predefinition since it represents a variety of objects, which have the same shape, but may have completely different cross sections. Moreover, such an unspecific element may gain symbolic character in a sketch through the surrounding elements. Omitting certain geometric information also enables a variety of functional interpretations. In case of the track, the depiction of a cart nearby actually classifies the line as a track (the same applies vice versa - the depiction of the cart is nothing but just a dot without the track next to it). Similar to a sentence, elements gain meaning by their combination and this meaning may go beyond the sum of the elements' meanings. One may 
argue that the same applies to any kind of perception, such as viewing real objects or photorealistic representations of objects. Even then, expectations that result from experiences make us see more than there actually is. But a sketch is not only the representation of (future) reality; it is the conceptualization of reality. Thus, the relations between elements are not just "read into" the sketch by perception, but they are explicitly depicted. This is clearly shown in the use of elements that only represent relations, such as auxiliary lines that indicate that certain elements are aligned on the same level (see Figure 4-17 on page 91). These elements have no direct equivalent in reality and may be compared to prepositional phrases in language. Conceptual sketches show syntactical characteristics. Similar to language, sketches may tell technical stories due to the combination of their elements. However, in contradiction to spoken or written words, the syntax of sketches does not refer to the succession of elements, but to the nearness of elements to each other. Still, even distant elements may be referred to each other with the help of prepositional elements.

We have seen that sketches offer the possibility to make predefinitions that combine symbolic and schematic characteristics within one element. Besides schematizing, a designer may use another kind of geometrically oriented abstraction, which is vagueness. A wavy, roughly drawn line may be interpreted in several ways - there is more than one concrete shape that may be represented by such a shape (see Figure 4-16 on page 90). Therefore, such a shape may be used and interpreted as a placeholder for a variety of shapes. Unlike schematic depictions, vagueness does not omit certain characteristics, but it blurs them. This is a different principle of abstraction, since vagueness provides a margin for the specification of a shape. This kind of abstraction may again be combined with both other kinds of abstraction discussed before, which are schematic and symbolic predefinition. So sketches may offer various possibilities for geometric and conceptual abstraction - this abstraction may range from completely omitting characteristics to setting margins of various bandwidths for possible specification.

Not only it needs to be considered how information is represented in a sketch, we also have to pay attention to the way this sketch is created. Sketching with paper and pencil is a process of action regulation. The depiction of each element in a sketch requires motor activity in the form of movement of the hand. The hand's motions are performed sequentially and likewise, the elements of a sketch emerge sequentially. If we examine the depiction of an element in detail, we see that shapes emerge continuously in accordance to this motion. Therefore, a sketch alters its configuration constantly during sketching activity. The designer needs to visually perceive the sketch throughout the whole process of alteration in order to regulate the distinct motion of the hand. Thus, the configuration at sight may be interpreted in a different way over and over again. Moreover, visual perception tends to reinterpret, even when the configuration does not alter (ARNHEIM 1977). In contradiction to the process of depiction, perception should not be regarded as a sequential, but as a more comprehensive activity. The application of the communication model from SHANNON \& WEAVER (1949) takes this distinction in account. For visual perception, the whole sketch is on display and there is no primary direction of "reading" provided by the sketch. Relations between elements can be established in every direction. Due to the syntactical characteristics of sketches, the conceptual meaning of a sketch can be changed significantly through reinterpretation. 
Another aspect of sketching as a process of action is the frequent occurrence of pointing into the sketch with the help of the pencil. Thereby, both the levels of real and imagined depictions may be connected to each other. The pencil's tip creates a focal point for the eye so that imagination can be integrated in the sketch and related to the configuration that is depicted already. Depiction and imagination can form a consistent, joint picture that may be estimated and further processed. Gesturing within the sketch may add yet another level of predefinition. It uses the "afterglow" of the motion as perceptible depictions that fade afterwards. Motor activity within the sketch opens up different levels of predefinition within one and the same medium and switching between those levels may be achieved without any delay.

Moreover, activity within the sketch adds another communication channel ${ }^{1}$ to the sketching process. The kinesthetic perception of one's own motion may be seen as a communication channel as well. It may interfere with the other channels, so the hand's motion and the pencil's motion respectively may be associated with depicted elements. Action within the sketch may evoke reinterpretation of the symbolic meaning of elements and thereby add clues for new conceptual ideas.

We see that the sketching process features a unique combination of characteristics that supports and enables creative processes of conceptual design. It is questionable whether these processes are initiated if not all of these characteristics are given. A future conceptual design medium should aim at providing similar advantages as paper and pencil while compensating for their deficits - such as the incompatibility with subsequent digital product models. In the next chapter, we will see how this claim may be settled by a digital design medium that links CAD and sketching: the 3D-sketcher.

\footnotetext{
${ }^{1}$ in terms of a soliloquy process in the style of communication according to Shannon \& Weaver (1949)
} 


\section{Future Media and Tools for Conceptual Design}

Sketches may be powerful devices in support of conceptual design in mechanical engineering. This unique design medium may even enable creative processes that lie beyond conscious control. Likewise, the moment of the occurrence of ideas for conceptual design solutions may elude from the designer's control. Incubation time is commonly quoted as a major characteristic of idea generation. Ideas seem to emerge only after an unsuccessful occupation with the problem - just when the person does not count on the idea anymore. However, conceptual ideas in mechanical engineering are usually no conceptual solutions yet, but they may be a clue or a starting point for the development of such a solution. In order to develop a solution through sketching, the designer only needs a piece of paper and a pencil -there is probably no design medium that is as freely disposable as a conventional sketch.

The heading of this chapter shows that we will consider media that differ from conventional sketches. Keeping in mind the subject matter of the previous chapter, the question comes up whether or not new media for conceptual design are necessary at all. At first, we will consider the processes and media that are subsequent to conceptual design. Thereby, we will find out that there are distinct arguments for new media. $\mathrm{CAD}$, as the provider of the universal product model, has the most important influence on a future conceptual design medium. It represents the interface to all other design tools and production processes. Therefore we will derive some basic requirements on future conceptual design media from CAD. Yet, the essential demands on the operational mode of future media arise from the consideration of conventional sketches. We will discuss these requirements and demands, which will be on a rather abstract level. In order to show that these demands can practically be realized through a new design medium, we will finally present the concept of the $3 D$-sketcher. This digital sketching device has already been set up as a prototype at the Institute of Product Development at the Technical University of Munich.

\subsection{CAx - A Necessity in Product Development}

Typical design processes in mechanical engineering as well as in kindred branches of engineering are supposed to result in the production of a spatial object. The input information for this production needs to describe processes of manufacturing and assembling that are capable of bringing a 3-dimensional object into being. There are two basic technological trends in engineering design, which have been employed for years already and which result directly from this demand. On one hand, the information that initiates production tends to be digital data that is fed into the production process directly. Automation in production has led to computer-aided manufacturing (CAM), which requires digital control data. With the help of appropriate interfaces, a digital product model (if existent) can be transferred into manufacturing data. Thus, the consistent application of CAD may rationalize the initiation, as well as the execution of production in mechanical engineering. On the other hand, CADmodels tend to be 3-dimensional in order to represent spatial products. Certain shapes, especially free-form surfaces, can hardly be represented just by 2-dimensional views and 
sections. In some branches, such as automotive and aeronautical engineering, 3D-CADsystems are certainly common.

The results from the surveys presented in chapter 3.2 estimate both these trends. The second survey showed that $87 \%$ of the designers (see page 46) use a 3D-CAD-system, nevertheless, $48 \%$ (also) use a 2D-CAD-system. So there still seem to be applications for 2-dimensional representations of products or at least parts of them. The surveys have also shown that sketches are often used before and during work with CAD and that they seem to fulfill different functions than CAD. The development of ideas was named as a major purpose of sketches, while the concretion of solution was stated as a major purpose of CAD (besides communication and presentation, see page 52). In the preceding chapter, we have comprehensively studied reasons for the unique suitability of sketches for the development of conceptual solutions.

Going back to the beginning of this thesis, we may recapitulate three characteristics of preliminary, conceptual solutions (see page 6). The preliminary character of concepts may consist in its incompleteness, irrationality and/or abstraction. As a typical example, we may once again consider a sketch that has been generated during the experiments and compare this sketch with a CAD-model.

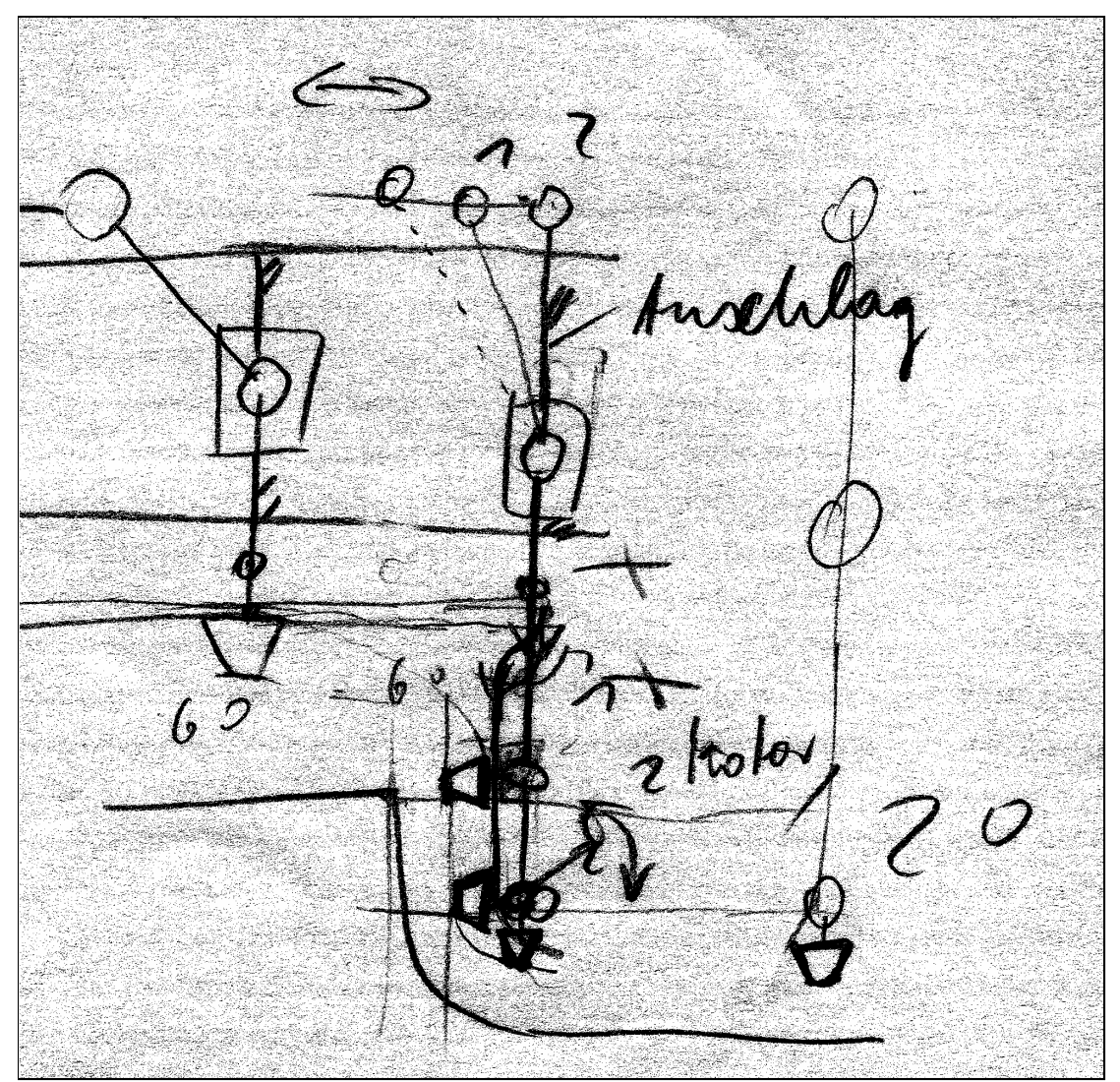

Figure 5-1: Characteristic design sketch 
The element on the right in Figure 5-1 is represented rather slightly with the help of thin lines and this element reflects all the characteristics of preliminarity we just mentioned. It is "floating in space" and not specifically attached to rest of the assembly. This representation is incomplete, because some relations within the product model are left out - the model is inconsistent therefore, yet the sketch can still represent it. In contradiction, typical CADmodels need to be consistent within themselves. Gaps within the model of an assembly need to be specified and some gaps within the model of a part even cannot be modeled at all (such as missing edges or surfaces within a solid body).

Moreover, the element in the sketch is irrational from a functional point of view. The part cannot reach any position to the right of the element that is represented by the word "Anschlag". We may suppose that this irrationality is introduced on purpose by the designer in order to represent the problem he works on. Such an irrationality differs from a simple mistake because the provisional character of this problem is indicated by the thin lines used for its sparse depiction. While using paper and pencil, the designer can vary the line thickness and (what is more important) the contrast of lines by applying different pressure to the paper. CAD-systems do not allow for such a direct representation of the provisional character of predefinitions.

Finally, sketches can represent abstraction in a far greater bandwidth than CAD can do. The sketch in Figure 5-1 shows elements that are abstract since they are schematic, symbolic/verbal or vague. Single lines represent voluminous objects, but omit shape and dimensions of its cross-section - this kind of schematic depiction is even predominant in the sketch. Schematic predefinitions may be the preliminary stage of more specific voluminous elements that follow in the course of the design process. CAD-systems allow for the depiction of lines as well, although these lines are usually not meant to be equivalent to volumes, but only to serve as auxiliary elements. Yet, those lines may be used as schematic predefinitions, from which more specific volumes are developed. CAD-systems do not provide the use of verbal elements such as the word "Anschlag", which serves as an independent element within the sketch in Figure 5-1. Likewise, the use of symbols in CAD is usually restricted to a limited variety of draft symbols; the application of an individual symbolic language usually is not supported. Yet, the representation of vague shapes through the use of wavy lines is extremely time-consuming when vector-based systems are used. Hence, it is not reasonable to work with geometric abstraction in CAD.

Sketches are seemingly much more suitable for the representation of conceptual design information than CAD-systems. However, sketches in engineering design may not be seen as conceptual design media which are independent from CAD. An empirical study by GÜNTHER (1998) investigated the change in levels of complexion and abstraction of the product definition during design processes. It has shown that there are designers that tend to complete the definition of a product as a whole on an abstract level before moving on to a more concrete level. On the other hand, it also turned out that other designers strive for the concrete specification of a part of the product before moving on to the next part, again starting on an abstract level. Figure 5-2 visualizes both these general approaches in a distinct way, although the differences between both these procedures may be blurred in reality. The temporary state of a product definition may be a mixture between different levels of abstraction. Switching 
between different levels of abstraction may be proceeded in both ways during design and it may refer only to a part of the product definition. After all, cognitive psychology also suggests moving up and down in abstraction in order to develop new solutions. Hence, an interface between sketches and CAD that works both directions would be necessary. Moreover, a medium that allows for the combination of conceptual design information and specific CAD-information within one product representation would be desirable.

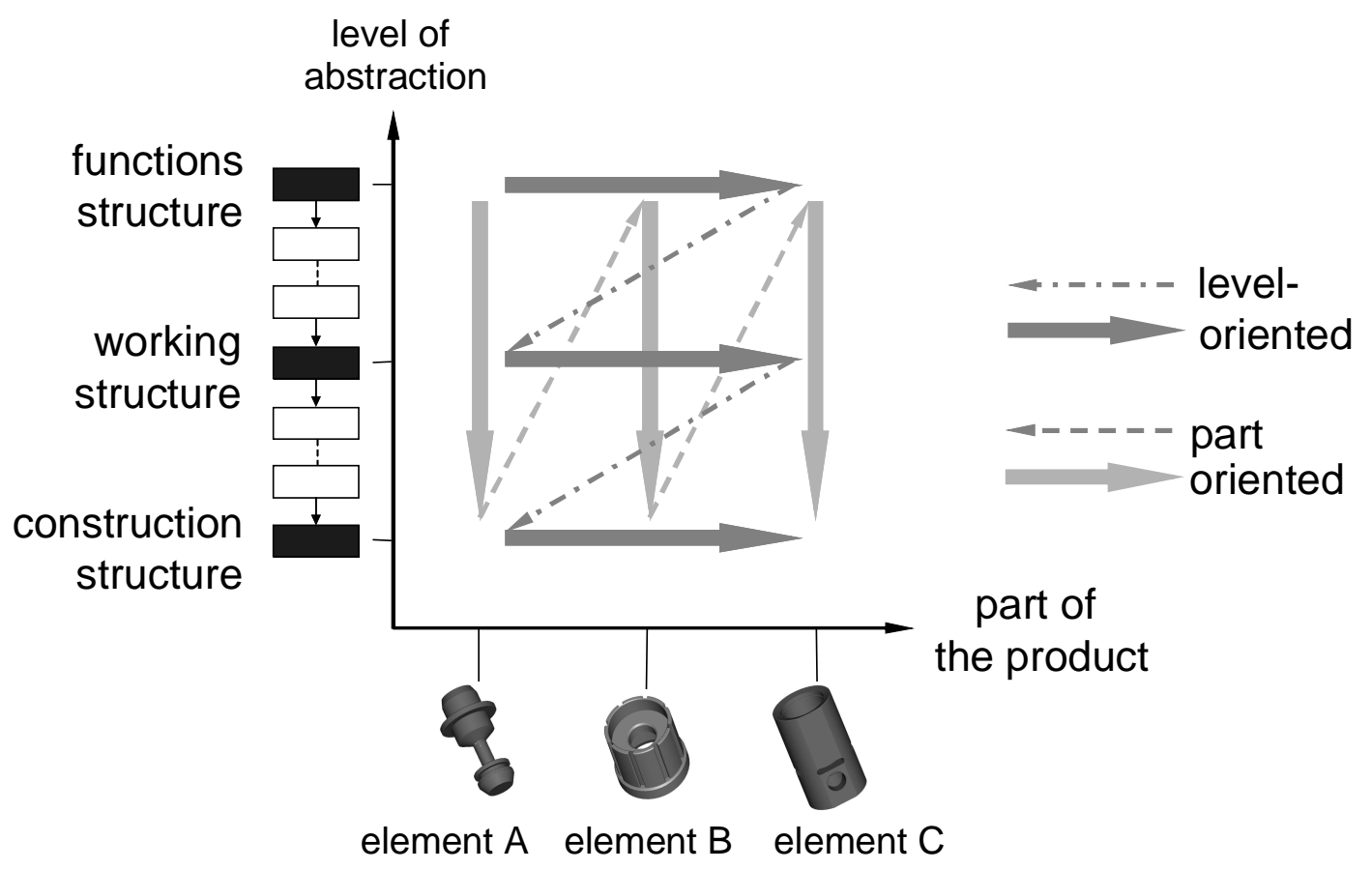

Figure 5-2: Different design procedures according to levels of abstraction and complexion

In case of CAM-based production, design data needs to be digital in the end of the design process. A design medium for mechanical engineering, which provides a mixture between conceptual and specific design information at least needs to provide such a digital output. An obvious idea for the realization of such a medium is the integration of the conceptual design sketch into CAD. Hence, sketched information will be digital and therefore accessible to digital product data management (PDM). This allows for establishing a product model that covers the whole lifecycle of a product. Moreover, 3D-CAD-systems provide spatial product models that are defined via coordinates in 3 dimensions. Likewise, 2-dimensional information from sketches has to be transferred into 3D sometime while being specified. However, if the sketch is fully integrated into 3D-Cad, then conceptual information could be 3-dimensional as well right from the start of conceptual design. A future sketching tool should combine the creativity-supporting characteristics of sketches with the advantages of digital system environments. In the following chapter, we will gather requirements on future conceptual design media, which come from the analogous and from the digital world. 


\subsection{Characteristics of Future Sketching Media}

On the basis of the insights into the use of free-hand sketches, several requirements on future sketching tools may be formulated. These requirements aim to enable the occurrence of the mechanisms that were worked out in the preceding chapter 4 .

1. A future sketching tool should not only enable the designer to use shapes of any kind, but also letters, numbers, words and individual symbols.

This is the basic requirement for achieving a degree of abstraction, which is as high as the degree that is provided by language. Such abstract elements can hardly be portrayed, yet they can be put into a spatial-relational context together with other specific elements.

2. It should be possible to combine geometrical and symbolical meaning in one element (e. g. a curved arrow to indicate motion along a certain direction).

Thereby, not only technical components may be represented but also technical processes. By combining shapes and symbols, "graphical verbs" may be formed and highly complex "graphical sentences" may be made up.

3. The designer should be able to depict ambiguous, vague shapes that may represent a variety of different concrete shapes.

Vague depictions blur the shape, as well as the position of elements. One possibility to achieve this kind of geometric abstraction may be the use of wavy and roughly drawn lines that allow for various interpretations. Another possibility is to draw multiple lines, which then cover an area of the design space, although they are meant to represent only one element. Somewhere within the indicated space, the future element will be located, but it is not yet specified where exactly this will be.

4. Schematic representations, which omit certain kinds of attributes, should be practicable (such as omitting shape and dimension of the cross section of a body by using only single lines).

This kind of abstraction is frequently used during sketching for conceptual design and seems to be a central form of representation. It may easily be combined with vagueness through the use of wavy lines or multiple lines.

5. The depiction of elements and parts of elements, respectively, is to be made sequentially.

In order to increase the chance upon creative re-interpretation by visual perception, the configuration of a sketch needs to change as often as possible. When all entities of a sketch are created sequentially, then each new element can be associated with the others one-byone. Due to the linguistic character of sketches that contain symbols, words and shapes, new contextual meanings can be read into sketches. 
6. Single elements should be created continuously under constant control of the designer.

Ambiguous shapes are not made up of regular standard shapes; otherwise there would probably be no ambiguity about their interpretation. When elements are created continuously, then the designer can access a sheer infinite variety of non-standard shapes. This way, ambiguous shapes and various individual symbols may be created freely. When the creation of shapes is controlled by motor-activity, then there is also a haptic feedback and a sensory-motor feedback from the motion. That feedback may be associated with the sketch and may also be a source of creative re-interpretation.

7. The area of perception and the area of action should be congruent with each other.

Action within the perception space allows for pointing at elements of the sketch and thereby supports the combination of imagination and real depictions. Gestures within the perception space may provide a separate level of preliminary depictions. When action and perception space are one, then the designer can change from pointing and gesturing to drawing directly without any translational effort and delay.

Beyond these demands from the insights into classical sketching, there are requirements that result from the subsequent $\mathrm{CAD}$-processes and data management processes. Digital sketching especially makes sense when all opportunities, which are offered by the digital world, are used.

8. The future sketching tool should create information that corresponds with 3dimensional product and process models.

Products in engineering design are mostly spatial objects and the processes that are necessary to produce them usually have 3-dimensional characteristics as well. CAD supports the creation of 3-dimensional product models. Yet, the definition of 3-dimensional geometries with the help of sketches can be complicating. Standard-views and cross sections are not capable of representing all imaginable 3D-shapes, but sketching in perspective requires special drawing skills. Still, a sketch in perspective only represents one view, while CAD-models can be rotated and provide all possible views on the spatial object.

9. The sketching process should provide a digital outcome.

A paper sketch that has been scanned and saved as a file generally meets this requirement; hence it may be managed by computer-based product data management. Yet, when the sketch information itself is digital, then subsequent processes based on CAD- and CAMtechnologies may access the conceptual information in the sketch directly.

10. It should be possible to contextually interrelate elements of the sketch with elements of a CAD-model.

When abstract elements of a sketch and the specific elements of the subsequent 3D-CADmodel are linked together, then it is possible to switch back into the conceptual state, even only for parts of the model. A digital sketch would even allow the mixture of abstract and specific information in one medium. 
11. Manipulation of a digital sketch should be possible in all those ways that are usually provided by digital design tools.

An entirely digital sketch would provide advanced processes of manipulation, such as rotating, shifting and up or down scaling. If the sketch features an data structure, then this holds for the sketch as a whole as well as for elements of the sketch.

We will see that the uncompromising transfer of the medium sketch and all its characteristics into a 3-dimensional, digital environment may provide an entirely new representational medium. It may not only enable completely new and more efficient design processes in mechanical engineering, but it may also be applicable to many other industrial, scientific and even artistic disciplines.

\subsection{The 3D-Sketcher - An Example of a Digital Medium for Conceptual Design}

The 3D-sketcher is the prototype of a digital sketching device using Virtual Reality (VR) that aims to fulfill the requirements on a future media for conceptual design (as discussed in the previous chapter). It may be regarded as a stand-alone medium (comparable to the classical paper sketch) but also as a module or even a feature of a future CAD system. We will discuss the possibilities of application, starting from the 3D-sketcher as an independent stand-alone system. This configuration has actually been realized as a prototype at the Institute of Product Development at the Technical University Munich.

\subsubsection{Sketching Lines in Space - The Basic Idea}

The very basic idea of the sketcher consists in the creation of 3-dimensional free-hand lines by the continuous motion of an input device through design space. The 3D-line is displayed within the design space, depicting the device's motion curve at the very location where that motion has taken place. It is displayed in real-time according to the device's motion. The device can be activated and deactivated by an appropriate control mechanism (such as a simple button). Figure 5-3 illustrates this principle - in this case, the device is actually the user's fingertip (we will see later on that this is possible). The 3D-sketch that emerges from this process appears to be floating in space and may be shifted or rotated in space with the help of an (additional) 3D-input device. 


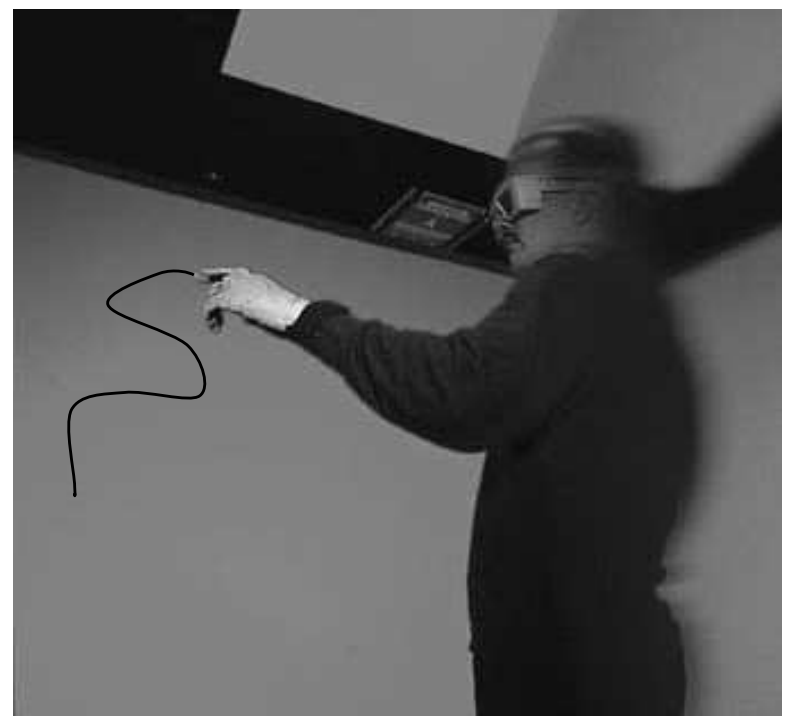

Figure 5-3: Drawing lines in space - the basic idea of the 3D-sketcher

The visual perception of such a sketch is truly 3-dimensional due to VR and is not comparable with the perception of lines within a normal 3D-CAD system. 3D-CAD may display objects in space according to the laws of perspective representation, but especially in the case of simple lines, CAD does not provide any practicable view. Just imagine two lines in space that do neither intersect nor they are parallel. In 3D-CAD without VR, these lines will always appear as a cross. It is neither perceptible which line is in front and which is in the back, nor what is the distance between them. So in case of 3D-objects that only consist of lines, truly 3dimensional representation is indispensable.

The principle of the 3D-sketcher provides the user with the free-hand line as the infinitesimal element of depiction. Thereby, a designer gains the most freedom and flexibility in representing any kind of context. The designer can depict shapes, illustrate symbols and even write words in space and put them into a spatial-relational context within the 3D-sketch. The continuous depiction process that is fully controlled by the user's motor activity allows for roughly drawn shapes that may represent a variety of concrete elements. Still, going far beyond the possibilities provided by paper-sketches, the design space is truly spatial. Making 3-dimensional depictions in that design space does not require special skills in perspective drawing; the user does not need to transform the angle and length of elements to the perspective view. He can draw a line in space as it actually appears. Figure 5-4 shows a 3dimensional sketch that was drawn by means of the 3D-sketcher. It illustrates a stand designed for the presentation of the 3D-sketcher at an industrial exhibition. The sketch is deliberately different from surface or volume-based models in order to retain the specific characteristics of the paper sketch in 3D. Still, it shows that surfaces and volumes can be represented by the 3D-sketcher. 


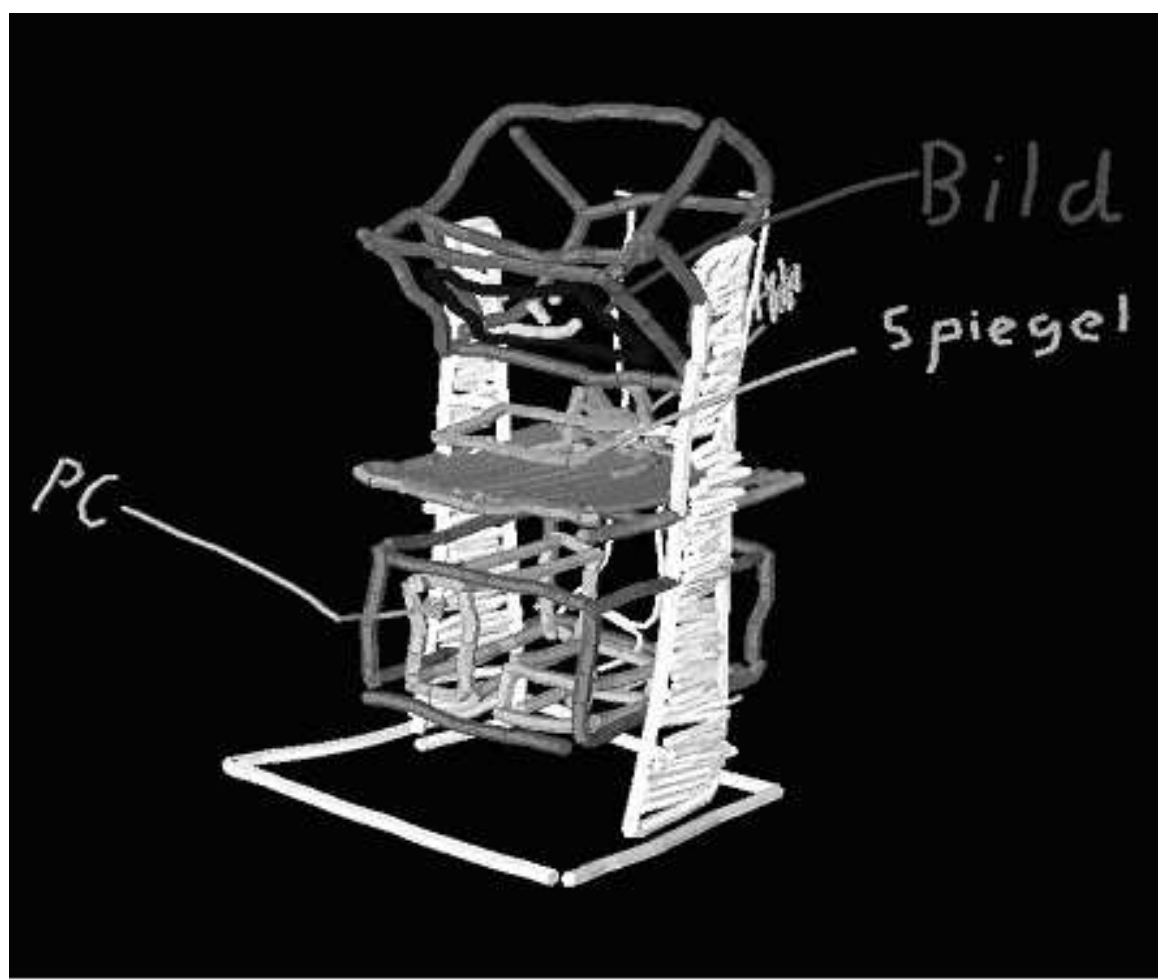

Figure 5-4: 3D-sketch that represents the stand for representation of the 3Dsketcher on an industrial exhibition

A structure or mesh made out of bended and buckled wires probably is the only equivalent to a 3D-sketch in reality. The wire frame model in CAD is somehow comparable to a 3D-sketch, although the creation process differs completely. The only metaphor to the creation principle of the 3D-sketch in the "real world" is the quick motion of a brightly shining object in darkness. In that case the motion line of this object lingers as an after-image on the retina of the eye and a line in space seems to be perceived. ${ }^{1}$

When considered as a conceptual design tool in mechanical engineering, it is essential to consider the way in which the sketcher may be linked up with subsequent CAD-systems. We will discuss this subject later on; to simplify matters, we will regard the 3D-sketcher as a stand-alone design media at first. Furthermore, this point of view broadens the horizon to think about the use of such a tool for other disciplines. Generally, every domain that designs 3-dimensional objects (e. g. architecture, electrical engineering, industrial design) or develops abstract contexts that may be represented 3-dimensionally (such as 3D-diagramms) may be taken into consideration. Artists may use such a media with no claim to represent any real

\footnotetext{
${ }^{1}$ Author's remark:

Sparklers, which just have gone out, but still glow, shine quite brightly in darkness. When glowing sparklers are waved in darkness, the resulting after-image remains rather long, so one could even write short words in space.
} 
object. The reader is invited to think about the possible use of the 3D-sketcher and the author would be glad to discuss any suggestion with the reader.

Several configurations that basically differ from each other may be used to realize the basic idea of the 3D-sketcher. At first, we will consider a low-cost version that aims to be applicable on a designer's workspace. From there, we will explore further possibility to realize the 3D-sketcher. Principally, the following operations and processes need to be provided by any 3D-sketcher:

1. The position of an input device is tracked within the design space.

2. It is ascertained whether the device is activated or not.

3. If the device is activated, an infinitesimal line element in space is determined or an existing line is extended by a line increment respectively.

4. The line is displayed in space in relation to the position of the input device (under consideration of the user's view angle).

5. The sequence is started over again with 1 .

The first prototype of the 3D-sketcher was developed at the Institute of Product Development of the Technical University Munich with the help of a Virtual Reality system. Although the prototype has been further developed since then, we will consider its initial state as the most simple and flexible configuration of this medium. This system is desktop-based so it made use of existing working environments of designers. A PC-based graphics workstation ${ }^{1}$ with a high-performace monitor is used. 3-dimensional visualization is generated by stereo view principle. The monitor provides both the views for the left and the right eye in an alternating manner with a frequency of $120 \mathrm{~Hz}$. Shutter goggles ${ }^{2}$, which are controlled by an infrared emitter, turn both eyes blind (and seeing respectively) in the same alternating manner. Therefore, each eye only perceives a view with a resulting frequency of $60 \mathrm{~Hz}$ (which is hardly sensed) and the represented object appears to be truly 3-dimensional. Software-base for the prototype of the 3D-sketching tool is a Virtual Reality programming platform ${ }^{3}$. For input, the force-feedback device Phantom Desktop ${ }^{4}$ is used.

The Phantom Desktop is basically a pencil-like handle with a button that is attached to the main device by a system of levers. The handle can be moved in space and is localized according to six degrees of freedom by means of sensors that are integrated in the lever joints. Thereby, the Phantom may be used as a very simple tracking system for the pencil-like handle. In case of the 3D-sketcher, this "space pencil" is used to draw the lines of the 3D-

\footnotetext{
${ }^{1}$ Fujitsu-Siemens Celsius 460

${ }^{2}$ Crystal Eyes by StereoGraphics Corporation (www.stereographics.com)

${ }^{3}$ Sense8 World Tool Kit 9 (this software works with Microsoft Windows NT 4.0). Microsoft Visual C++ 6.0 developer studio was used to implement the 3D-sketcher into this platform.

${ }^{4}$ Phantom Desktop by Sensable Technologies (www.sensable.com). Ghost SDK, a developer kit that is shipped with the Phantom, was used to implement this force-feedback device.
} 
sketch. As long as its button is pressed, a 3-dimensional line appears right at the current position of pencil's tip. This effect is achieved by a special configuration of hardware elements ${ }^{1}$. The configuration of the 3D-sketcher is presented schematically in Figure 5-5. The user watches the display of the monitor via a semi-transparent mirror that is placed horizontally over the designer's worktable. Thereby, the 3D-sketch that is displayed on the monitor in stereo-view seems to be floating in space underneath the mirror. At this location (which is actually the design space), the Phantom is placed, which can be seen through the mirror as well due to its semi-transparency. All components of the system are adjusted to each other in a way that an emerging 3D-line is perceived in real-time at the very tip of the pencil so action-space and perception-space are congruent.

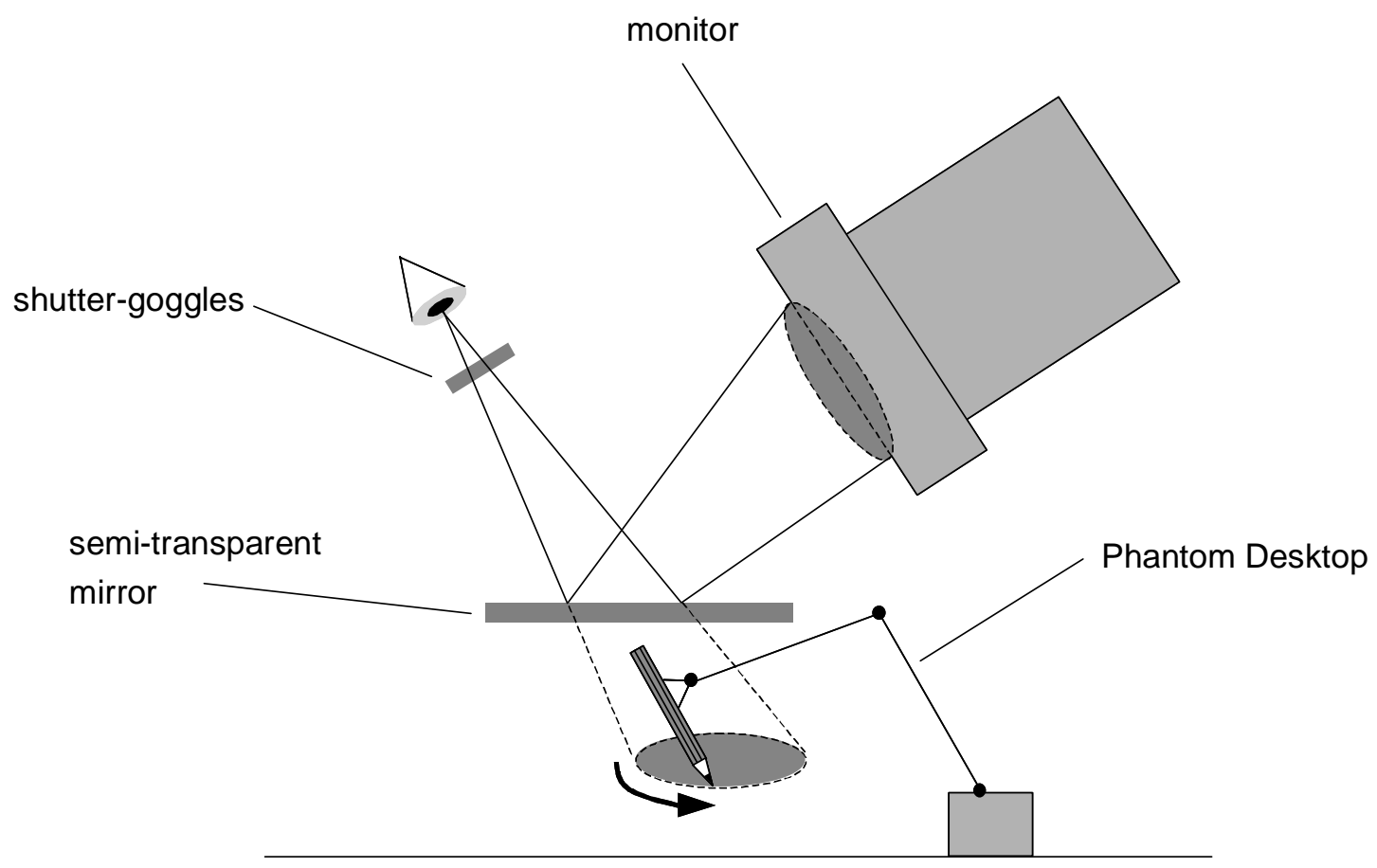

Figure 5-5: Configuration of the 3D-seketcher (similar to the Reach-In-Display ${ }^{l}$ )

The stereo view that is provided by the system is adjusted to the position of the user's head and to the user's angle of view as well. With the help of a tracking system, position and direction of the shutter goggle are ascertained and the represented views are adapted accordingly $^{2}$. So the user can regard the sketch from different angles and explore its 3dimensional structure by altering his view, which should meet natural habits.

\footnotetext{
${ }^{1}$ The configuration is similar to the Reach-In-Display by Reachin Technologies AB (www.reachin.se)

2 The author has to admit that at the present time, this functionality is provided only theoretically. The magnetic tracking system is unfortunately interfered by the magnetic field from the monitor. Therefore, the adaptation of
} 
In comparison to a direct view on the monitor, the indirect display of the 3D sketch via semitransparent mirror provides two major advantages. Due to this configuration, the user's hand is visible in the design space but still does not obstruct the view on the sketch. The second advantage arises from the fact, that the best impression of spatiality is achieved when the virtual object seems to be located right in the monitor plane. In case of direct display, the perception-space and action-space could not be congruent, because then the user would knock against the monitor. With the help of the mirror, the area of best 3D-impression is transferred to the free space underneath the mirror.

The lines that emerge while sketching are stored internally as a "voxel ${ }^{1}$ cloud." This corresponds to a pixel graphic used for example by software for photo processing. The line consists of a number of cylinders that are lined up in space according to the motion of the pencil. In this aspect, the sketch deliberately differs from CAD-models, which are usually vector-based. At first, the voxel cloud does not contain any semantic information concerning lines or shapes that might be formed by related voxels. Still, all the voxels that evolve from one continuous sketching motion (when the button is constantly pressed) can be annotated internally as being related to each other. Such an accumulation of related elements is then regarded as being one line.

The sketch can be shifted and rotated in space as a whole with the help of any 3D-navigation tool (such as a space mouse for example). Thereby, the sketch can be inspected from different sides. The area in which the user actually wants to sketch can be turned up front. Experiences with the 3D-sketcher showed that certain directions of the hand's motion were less likely to depict accurate lines than others. For that matter, the sketch can be rotated in a way that the favorable direction is always at hand. This corresponds to the possibility to move and turn the piece of paper on the desk in case of normal sketching. These functions can be operated by one hand while the other hand is used to sketch. With the help of a space mouse, the sketch can also be zoomed, which means that its size is scaled up or down ${ }^{2}$. The same functions (moving, rotating and zooming) can also be applied to a single line within the sketch only. Furthermore, single lines may be deleted. All these functionalities may be activated through keyboard commands. In analogy to the paper sketch, eraser functionality is implemented in the 3D-sketcher. When the sketching mode is switched over to the eraser mode, then single voxels of the 3D-sketch are deleted as soon as they are touched by the pencil tip while the activation button is pressed.

the stereo views to the user's position works in an unstable manner. In future, this problem will be solved with the help of an optical tracking system.

\footnotetext{
${ }^{1}$ The word „Voxel“" contains ,,volume“ and ,pixel“"

2 "Zooming into and out of a model", as it is known as a function from CAD, corresponds with two different functions in case of the 3D-sketcher. A 3D-sketch can be moved to the front of the design space so it is closer to the user. Still, the sketch does not alter in size. Furthermore, the sketch can be scaled up and down without being moved within the design space. In case of CAD, both these functions cannot be distinguished.
} 


\subsubsection{Further Functionalities and Features of the 3D-Sketcher}

The initial prototype allows for changing the characteristics of the line that is displayed, such as color and thickness, through a menu. However, it may be supposed that the line thickness is an essential kind of expression that is enclosed in the lines of a sketch. "Weak" lines may imply a provisional character of the predefinition that is represented by the line (see page 93). By contrast, "strong" lines may emphasize depicted elements. Therefore, it is desirable to vary the strength of a line intuitively and simultaneously during the sketching process. In "real" sketching, this possibility is given through the variation of the physical strength used to press the pencil-tip on the paper. Depending on the pencil that is used, the harder it is pressed on the surface, the darker and thicker the line becomes. In case of the 3D-sketcher, the pressure principle cannot directly be applied since in the design space, there is no surface that may prop up the pressure from the pencil (however, a virtual one may be created - we will see later on). It is conceivable to provide the pencil with a pressure-sensitive button for sketching activation; the harder the button is pressed, the stronger the line emerges. A special slide control knob may fulfill that purpose, too; the strength of the line would relate to how far the slide is pushed.

In general, it may be supposed that the "strength" of a line should correspond to the perception of the intensity of the contrast between the line and the background. For that matter, a digital sketching tool may offer two ways to vary the line's contrast: thickness and color intensity. In case of any sketching tool, the color intensity should be varied in the first place. Thick lines automatically reduce the achievable precision of depiction, but the variation of several shades of grey up to deep black varies the intensity of perception while still providing the same line thickness. The author supposes that the proportional variation of color intensity along with a slight variation of thickness may bring about the most "natural" feeling of contrast between line and background. Figure 5-6 shows three ways to vary the contrast of a line. The designer can still achieve a certain line thickness by drawing a line repeatedly over itself. Slight divergences in position and direction result in a thick line - we have already observed such a process for classical paper sketching during experiments (see page 97).

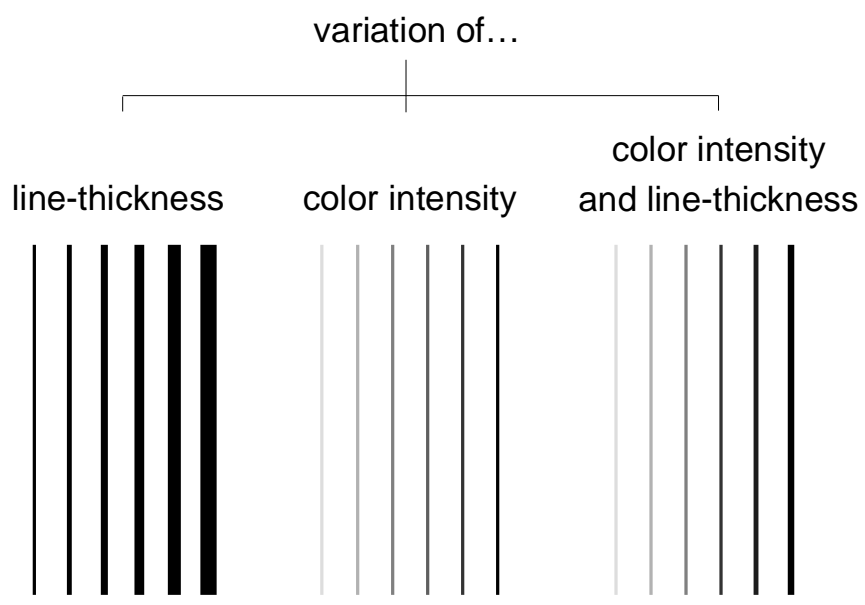

Figure 5-6: Variation of the contrast of lines 
The prototype of the 3D-sketcher, as it is described in the previous chapter, does not take advantage of the force-feedback functionality of the Phantom device. Still, there are certain possibilities to use this opportunity for the sketcher in a practical way. It might for example be useful to create a virtual plane within the design space on which the designer can sketch. Force-feedback can prevent the pencil-tip from moving through the plane, so for the user, this plane appears to be there physically. He can sketch on the surface of the plane as if there is a drawing board in the design space. Figure 5-7 illustrates the principle of the virtual drawing board. Such a semi-transparent board may be switched "on and off" by the designer and may be placed freely in the design space with the help of the space mouse. If the designer anticipates that he will only depict elements within one plane for a while, then the virtual board may be a helpful feature. The board might also be an assisting feature for users that are not familiar with the 3D-sketcher. With the help of this board, a 3D-sketch can be created even though the user still sketches in the classical way. Instead of drawing freely in space, the user keeps on adjusting the virtual board within space and then sketches in that plane. Thereby, the procedure of drawing 3D-sketches may be explored stepwise without losing complete reference to classical sketching. In case of the virtual board, the common way to vary the contrast of a line (by variation of the pressure applied to the sketching surface) can be adapted. However, the force-feedback device needs to measure the forces applied by the user through sensors.

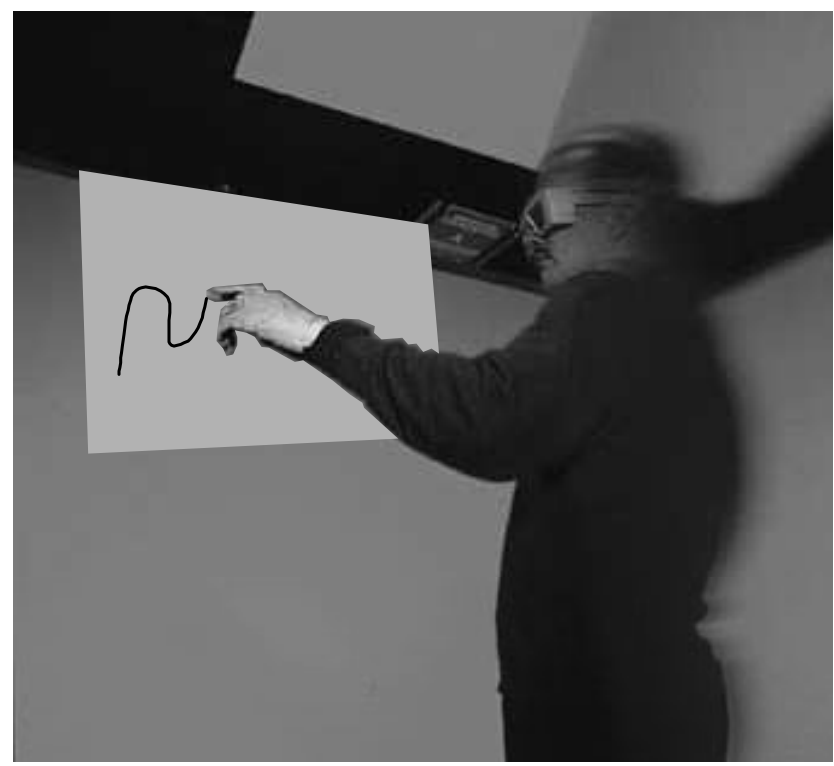

Figure 5-7: Virtual drawing board

Force-feedback can also be used for a "snap"-function that pulls the pencil-tip to the end of existing lines as soon as the tip gets close to them. This functionality may support the designer in meeting end points exactly in order to draw continuous sequences of lines and shapes. Such a snap-function may also support the designer in meeting the points of a virtual grid, which would be the equivalent to squared paper. 
Other virtual objects, consisting out of bodies and planes, may be displayed within the 3Dsketch and may be made tangible in the sketch due to force-feedback. Thereby, the designer can sketch within the space between these virtual objects. Force-feedback physically prevents him from sketching beyond that space, so he cannot penetrate those objects. It is possible to sketch on the outer (or even inner) surface of such virtual objects just as if they were real.

\subsubsection{Different Hardware Configuration of the 3D-Sketcher}

Pencil-like input devices for VR (with or without force-feedback) can usually be tracked in space according to their position and direction. For the use of such a device as 3D-pen, it is only necessary to localize the position of the pencil-tip regardless of its direction. Still, for activation and deactivation of the sketching mode, another input possibility is necessary. In case of the Phantom, this is a button that is integrated in the handle. However, VR technology offers further, more diverse possibilities for data input.

With the help of a dataglove, all gestures of the user's hand may be recorded along with its position and direction. Thereby, the glove can turn the user's fingertip into a 3D-pencil. Furthermore, it allows the user to control a number of different modes intuitively. Drawing lines and erasing lines can be distinguished by certain pointing-gestures (with one and two fingers respectively). Thereby, the posture also determines the "pencil-tip". A grasping-like gesture of the whole hand may indicate the intention to shift or rotate the whole sketch (so a dataglove might replace the space-mouse for the other hand as in case of the prototype described before). When only thumb and forefinger are used for a grasping-like posture, this may select and move just a single line. Zooming the whole sketch may intuitively be controlled by a "come closer!" gesture and a "back off!" gesture respectively. The virtual drawing board can be generated by a wiping-gesture, the board can then be moved in the same manner as a single line. Mixed use of different input device for each of the user's hand may be useful as well: e. g. depicting lines with the help of Phantom with one hand (in order to use force-feedback) and moving the sketch by means of a dataglove with the other hand (for most intuitive control). Gesture control can even be complemented by voice control.

For visual data output, there is a large variety of different devices that provide very distinct characteristics of use. Workbenches, Head-Mounted-Displays (HMD) and caves need to be considered besides desktop-VR systems (as used in case of the initial prototype). A workbench enlarges the design space many times over, but this device probably does not fit into a designer's workspace. All the more, a configuration with a semi-transparent mirror (as in case of the prototype) is nearly impossible to realize. Accordingly, perception space will need to be in front of the screen and visual distortion will be the consequence. Still, such an enlarged design space may allow for two people working on the same sketch simultaneously ${ }^{1}$. This configuration will be useful for collaborative idea generation or design reviews when sketching serves mainly for communication purposes, but not for accurate and detailed

\footnotetext{
${ }^{1}$ In that case, the monitor/screen needs to display four different pictures in an alternating manner with a very high frequency - two stereo views for each user. Each goggle turns either each of the user's eyes or both eyes blind in a certain sequence, so each eye only perceives its corresponding view.
} 
solution development. Different configurations of the 3D-sketcher (even the development of a mobile sketcher) are considered by MüLLER et al. (2003).

The characteristics of the use of workbenches for 3D-sketching apply to the use of caves as well. Moreover, the design space could be all around the users, so they could be immersed in the virtual scenario and still sketch within the scenario. This configuration requires very much space and cost, though. In case of the use of a HMD, less space is required and immersion within the scenario is given as well, but the application would be limited to only one person in the first place.

\subsubsection{Integrating the 3D-Sketcher in a CAx-Environment for Engineering Design}

Beyond technological improvement, it is essential for future application of the 3D-sketcher either to integrate this medium into existing working environments or to create new, practicable environments for it. In the case of design in mechanical engineering and kindred branches, the 3D-sketcher should be part of actual CAx-based development processes and IT environments. This concerns particularly the linkage to or even integration into existing CAD systems. Concepts that are represented in sketches may be concretized to CAD-information and abstract sketches may again be deduced from CAD-models. A bi-directional interface between both these media would be necessary for that purpose.

It is possible to convert a CAD-model into a VRML-file, which then may be loaded into a VR-scenario such as the 3D-sketcher. An appropriate interface is provided by most of today's 3D-CAD-systems and thereby, the way back from CAD into the sketch is realized. On the one hand, this transfer can be used to conceptualize a solution approach that has been specified in CAD before. The VRML-object can be used as a pattern or template for conceptualization within the 3D-sketcher-environment. Afterwards, the initial VRML-file may be deleted and the abstract sketch remains. On the other hand, existent boundary condition for a future conceptual solution can be loaded into the sketching environment. Thereby, a new concept may be fitted into the available design space right from the start. Objects that surround the design space may even be modeled physically with the help of force-feedback, so the pencil cannot penetrate those objects.

The transfer of information from the sketch into CAD is probably much more complicating than the reverse way. CAD-systems process vector-based data, but data from the 3D-sketcher is voxel-based. Though voxels may be represented as a multitude of small vector-based solids, the size of the CAD-model would extensively increase and it might be hard to handle by means of data processing. CAD-systems would need to be adapted in order to provide sketch-like information within a CAD-model. Another possibility to integrate information from sketches into CAD would be the conversion of sketch-like data prior to CAD. Fuzzy lines could be straightened within the 3D-sketcher before transferring them into CAD. The concretion of voxels into vectors could be supported by means of software assistants, but the appropriate procedures and algorithms need to be developed yet. Principles from so-called "fuzzy logic" could be adapted for that purpose. Approaches for "merging" the 3D-sketcher and CAD will be considered in MüLLER (2004). 
In the long run, it would be desirable to combine 3D-CAD and the 3D-sketcher within one medium. Transferring data from a sketch-like state into a specific CAD-model would not require a change of medium and format anymore - the data would only be processed contextually then. For this matter, CAD would need to become truly 3-dimensional with the help of VR-technologies. Since models from 3D-CAD are spatial objects anyway, this step would just be logical. The input-devices from 3D-sketching could be used for CAD-work as well. Moreover, if CAD becomes a VR-application, then such a working environment would need to allow hours of work without immoderate mental and physical fatigue. This requires

- the appropriate combination of VR-hardware for each particular situation (task, branch, user, etc.),

- the availability of high-performing components,

- the development of software that provides ergonomic procedures for the operation of CAD,

- the integration of the whole configuration into the common workplace of a designer and

- a price for such a configuration that is economically maintainable.

The initial prototype of the 3D-sketcher has not been suitable for industrial application yet, but it is the basis for further development in order to achieve this aim. Nevertheless, it is usable for other purposes than engineering design already. First of all, the 3D-sketcher is a representational medium that may freely be used for artistic matters. Future development of the 3D-sketcher will consider its application for other design related branches, such as architecture, civil engineering, industrial design and electrical engineering. 3-dimensional schematic diagrams may be used for communication about any subject in physics, chemistry and biology for instance. 
This book is available at Verlag Dr. Hut, München, www.dr.hut-verlag.de (ISBN 3-89963-176-5) 


\section{Conclusions and Consequences}

In this thesis, we have considered the use of paper sketches for conceptual design in mechanical engineering in order to create a future design medium. Conceptual design is a process that brings about the generation of any kind of technical concept. This process is an application of creativity, since in the end there may be a solution that has not been known to designer beforehand. To this day, that kind of creativity is only a human ability. Still, designers are under the necessity to receive external support in order to take advantage of this ability.

Limitation of memory capacity clearly urges the human mind to apply any kind of principle that relieves the crucial "working memory". Thereby, the structure of data storage within memory suggests several basic mechanisms of load reduction. The semantic net of the longterm memory provides the aggregation of information entities by abstraction: several specific elements that have certain features in common may be represented by only one abstract element. Beyond that, the separation into working-memory and long-term memory points to another mechanism of memory relief: outsourcing information into another medium that is used as an "overfall basin". Instead of learning information by heart (which corresponds to the transfer from working-memory to long-term memory), one may use an external medium as well, such as a sketch.

Abstraction does not only save memory space, it plays an important role in problem solving as well. By abstraction and subsequent specification of a concept, one may find a neighboring concept that differs from the original concept in certain essential features. By combining such concepts, one may find a new solution to a problem. Design may well be seen as problem solving. Changing levels of abstraction is regarded as a useful procedure in design reasoning. Such a procedure may be carried out in form of a dialogue with oneself - Dörner calls it "the inner conversation of the soul with itself'. Moreover, problem solving processes show such characteristics as recursion and iteration.

In case of goal-oriented work tasks (a design task in mechanical engineering surely is one), such problem solving processes need to result in actual objects. Hence, cognitive processes materialize themselves in processes of real action. So-called TOTE-units may be used to describe the execution of even complex tasks. The resulting series of actions is a gateway to the underlying structure of control processes, which in turn reflects cognitive processes.

Procedure plans from design methodology are supposed to support designers in processing complicated design tasks. Hence, such plans show similar characteristics as problem solving processes described in psychology. Still, such plans are rather generally applicable and provide an appropriate structure of sub-tasks to process. They provide only few hints on how to perform specific action in order to execute those tasks. However, design action is rather determined by the design media and tools that are used.

In this thesis, we have distinguished between media and tools for design. Tools facilitate the processing of product definitions, whereas media represent product definitions. Representing a definition may not be seen as just one specific way of processing it. Due to the limitation of 
human memory capacity, most product definitions in design would not even be achieved without an external medium. Hence, one may assert that the product representation may be equated with the product definition. Tools can only help to process the definition that is represented by the medium in the first place. CAD certainly provides numerous tool-like functions. Still, these functions access a product definition that is represented by CAD. CAD's characteristics as a medium strongly differ from the characteristics of sketches. Since CAD provides a digital outcome that may be used in subsequent processes of production, this medium is indispensable for today's design processes in mechanical engineering.

The surveys among designers from industry, which are presented in this thesis, point out that $\mathrm{CAD}$ and sketches are integral parts of design. Both these design media seem to complement one another according to certain functions. The use of sketches mainly centers on communication and idea development, while CAD is rather used for purposes of concretion, verification and documentation of given solutions (besides communication and presentation). However, sketches and CAD do not complement each other according to the product attributes that are represented with the help of these media. CAD clearly reveals a decisive deficiency in representing certain indirect attributes such as aspects of strength and deformation for instance. However, sketches are rarely used to represent these attributes either. Revealingly enough, these attributes are named by most of the designers as being decisive characteristics of typical design tasks. Even Rapid Prototyping and Virtual Reality are not used to represent these attributes.

The surveys also reveal certain dissatisfaction of the designers with their CAD-systems. There is a minority of designers (which is not negligible, though!) that judges CAD as being downright negative according to the time involved and the quality of results when working with CAD on conceptual solutions. Sketches are rated more positive in this regard. Moreover, $\mathrm{CAD}$ is mostly rated mediocre according to intuitive operation, effectiveness of operation and effort for changing a design solution.

An experimental study has been presented that investigated the sketching processes of more than 60 students and designers, each working on two different conceptual design tasks. Statistical analyses have shown that the individual sketching processes of different persons may strongly differ. Yet, at least some similarities could be identified. All persons sketched longer in case of the more difficult task. Moreover, not only components, but also processes were depicted by the test persons. Lastly, most of the persons used at least three different modalities of information representation, such as visual-graphical, schematical, symbolical and verbal depictions.

These findings could be specified by further analysis of particular sketches. It has shown that technical elements may entirely be represented within a sketch even by words only. Thereby, information on a very high level of verbal abstraction may be integrated into the geometrical context of a sketch. The same applies to symbolical depictions. Yet, there are other ways to represent abstraction within a sketch. Schematic depictions omit certain classes of geometrical attributes and represent a variety of different specific objects. Moreover, shapes may be represented vaguely with the help of wavy, roughly drawn lines. Such depictions "blur" the specific geometry of a shape and provide a range of possible attributes. Hence, such depictions may represent a variety of specific shapes, which is a characteristic of abstraction. 
Also, such a vague depiction does not have any verbal counterpart that reflects its superordinated character in one term. Hence, abstraction within a sketch may be more diverse than abstraction that is purely verbal. Similar to language, sketches can express irrational information and thereby provide the formulation of a problem - as the starting point of a subsequent solution process.

In sketches, various kinds of abstract and concrete information entities can be mixed up. Parts of a rather specific solution may be abstracted and conceptualized at any stage of the design process when sketches are used. Therefore, conceptual design does not need to occur at the beginning of a design process exclusively, nor does it need to happen within a comprehensive and clearly distinguishable phase.

Moreover, the geometrical elements within a sketch show characteristics of linguistics. Relations between elements are provided not only by their spatial configuration. There are auxiliary elements that may establish relations between elements that would not be interrelated otherwise (e. g. because they are distant from one another). Such relations may not only be spatial, but may also be temporal (such as the numbering that indicates different states of a process). Such auxiliary elements are somewhat comparable to prepositional phrases in language. Likewise, depictions that represent objects may be regarded as nominal phrases and depictions that represent processes (such as motion arrows) may be seen as verb phrases.

A sketch offers element types that are quite similar to the ones that are provided by verbal language. Yet, it is even more important to point out that sketches show syntactical ${ }^{l}$ characteristics as well. An element of a sketch provides information not only from within. Moreover, it holds meaning in combination with its surroundings by the way it is integrated in the context of the whole sketch. Sketches literally tell technical stories by combining graphical verbs, nouns and prepositions. The message that is conveyed by a sketch depends on the way in which those elements are correlated with each other by the viewer. The syntax of a sketch is not as unequivocal as it is in case of verbal language, though. However, there are manifold ways to "read through" a sketch. Thereby, the viewer of a sketch may cancel old relations and establish new relations. In that case, the "order of words" within the graphical sentence literally gets mixed up and a sketch may make totally different sense than it did before.

Visual perception tends to re-interpret the configuration of shapes at sight. This is a prehistoric mechanism of protection, which tries to make sense out of a given situation over and over again - in order to discover the predator in disguise, for instance. It applies constantly, running unconsciously, so it eludes from deliberate control. In the context of sketching for design, this mechanism may have an effect that is beyond protection from wild animals, though. Re-interpretation of the configuration in a sketch may establish links between elements that were not meant to be related beforehand. We have seen one example of a sketching sequence in which this mechanism may have led to a completely new solution

\footnotetext{
${ }^{1}$ As a reminder: Roughly spoken, the syntax is the set of rules that determines the order of words within a sentence. The relations between the phrases of a sentence are determined by the order of the words.
} 
principle. Such an enormous impact is made due to the syntactical characteristic of the "graphical phrases" in a sketch. In order to briefly recapitulate this sequence: An arrow, which initially referred to a moving cart, may have been correlated with a track, which initially was supposed to be immovable. Hence, the new relation may have been interpreted as a track that moves in itself and so the initial concept was changed into a circulating belt. The "graphical noun" track has been correlated to the "graphical verb" arrow, which resulted in a new meaning of the sketch. The change of concept could only be so radical due to the high level of abstraction that was inherent in the elements of this sketch. Unfortunately, our explanation approach for this process could not be verified due to the experimental setting.

The mechanism of re-interpretation is even reinforced by the fact that information is externalized sequentially in a sketch, but internalized comprehensively. Hence, the sketch is ever changing while it is constantly internalized. This process can be appropriately represented by the soliloquy-communication-model that has been introduced in this thesis. It considers the use of design media as a process of communication with oneself. In this case, the sketch is used as a communication channel. The model allows for the differentiation between the sub-processes of externalization, storage and internalization of information during sketching. Those sub-processes are certainly interrelated, but they show very different characteristics, though. The model takes into account that the information, which is being "exchanged" in the course of such a communication, may be altered during each of these subprocesses. Thereby, the context and even the meaning of sketches may change.

Another intense case study has been carried out that has investigated the actions of a designer during sketching in great detail. It has shown that predefinitions may be made on different levels of depiction. At first, lines may actually be sketched on the paper. By contrast, one can also imagine the line of a fictitious sketch in the mind's eye. The differentiation between both these kinds of predefinition is rather consistent, but not all predefinitions fall in either one of those categories. A designer looks at his sketches throughout the whole sketching process and while he does so, he points at spots in the sketch with the pencil tip. The tip may guide the eye through the sketch and prevent it from straying, since it increases the contrast at that spot of the paper. On the one hand, such spots are characteristic parts of given elements, which seems to suggest that the designer analyzes and reflects the actual state of the solution. Still, the designer points at free spots of the paper just as often. This lays ground for the assumption that the designer imagines a future depiction right at this spot of the sketch. So this is another level of depiction that combines characteristics from both the levels named above. It allows for the direct integration of mental imagery into the actual sketch on the basis of active occupation with the sketch. However, action within the sketch opens up yet another level of depiction, which is even closer to real sketching. The designer performs gestures, which simulate the drawing motion without the tip actually touching the paper's surface. Such a gesture may be a trial-action for a real depiction, whereby the corresponding motion is perceived in a visual and a sensory way. Hence, the designer can actually perceive the shape of the future depiction, although there is no permanent consequence from such a gesture. In fact, the trace from the gesture resembles a line that "erases itself" after a while (unless it is constituted by a "real" drawing action). Still, this kind of predefinition was observed much less frequently than pointing action. 
All these levels of depiction are provided by the paper sketch and each of those levels has different characteristics. Moreover, the designer can switch between them without delay. As we have seen in the case study, the designer does actually apply those levels and thereby realizes a very fluent and efficient process of testing, dismissing and constituting solution variants.

In order to summarize the findings from the extensive experimental study, one may point out that sketching is a highly complex process that involves aspects from...

...communication - sketching may be regarded as a dialogue of the designer with himself

...perception - creative processes of (re-)interpretation generate new solution ideas

...problem solving - sketches provide very different levels of abstraction

... action regulation - different levels of depiction are enabled by sketching motion

...language - elements of sketches form graphical sentences on a high conceptual level

A future sketching medium should adopt the advantages from paper and pencil while compensating for their deficits. Moreover, it should provide other useful features as long as they suit the overall principle of the new medium. Since product information in mechanical engineering tend to be digital, it stands to reason to create a digital sketching medium, which fits into a CAx-environment then. Above all, the medium needs to interact with CAD, in a similar way as classical sketches do. A bi-directional interface between both these media would be desirable at first. On top of that, the integration of the sketch into CAD would be the next logical stage of development. Hence, the future sketch would need to be 3-dimensional in order to suit modern 3D-CAD-systems.

From the analyses presented in this thesis, we have derived a number of requirements on a future sketching medium in order to preserve the advantages of "classical" paper sketches. For instance, the medium should enable the depiction of shapes, words and symbols on any level of abstraction. We have supplemented these requirements with demands coming from the digital working environment in mechanical engineering. Manipulation functions, such as rotating and shifting single elements of the sketch should be provided, for example.

The requirements are met by the 3D-sketcher, which is the functioning prototype of a digital and truly 3-dimensional sketching medium. The basic idea of the sketcher is to create 3dimensional free-hand lines by the continuous motion of an input device through design space. Due to the application of Virtual Reality technology, the emerging line appears to be floating in space at the very location where the motion has taken place. The designer uses the free-hand line as the infinitesimal element of depiction and gains the most freedom and flexibility in representing any kind of technical context.

The prototype is a desktop-based system that aims to fit into a designer's working environment. It uses a special monitor setup, shutter goggles, the phantom input device and a space mouse. The pencil-like grasp of the phantom is moved in space with one hand, while the other hand operates the mouse in order to rotate, shift or zoom the sketch or even parts of it. The force-feedback functionality of the phantom might be used to physically represent virtual objects or planes (which may serve as virtual drawing-boards). 
Different hardware-configurations allow for different operation modes and applications. The user's fingertip may be turned into a 3D-pencil with the help of a dataglove, for instance. It would also allow for a highly intuitive operation of the system by gestures. A workbench would provide a much larger design space, while a cave allows for the immersion of several persons into the sketch. Thereby, the sketcher may as well be a useful device for depiction in several disciplines other than engineering, such as architecture or even arts.

In the long run, the sketcher should be fused with CAD in one single medium. As a consequence, $\mathrm{CAD}$ needs to become truly 3-dimensional, too. Thereby, operational devices and principles from the sketcher could also be used to operate CAD in a more intuitive way. Nevertheless, the future configuration would need to meet certain economic and ergonomic requirements. The prototype that has been discussed in this thesis might be the starting point of research towards this objective. 


\section{References}

AMFT, M.:

Phasenübergreifende bidirektionale Integration von Gestaltung und Berechnung.

München: TU, Diss. 2002.

ARNHEIM, R.:

Visual Thinking.

Los Angeles: University of California Press 1969.

ATKINSON, R. C.; SHIFFRIN, R. M.:

Human Memory: A Proposed system and its Control Processes. In: Spence, K. W.; Spence, J. T. (Eds.): The Psychology of Motivation and Learning. Vol. 2

New York: Academic Press 1968.

\section{BACH, M.:}

Hering Illusion

Date of access: 01.01.2003, URL: http://www.michaelbach.de/ot/ang_hering/index.html

BAILEY, R. W.:

Human Performance Engineering.

New Jersey: Prentice Hall 1989.

BARTL, C., DÖRNER, D.:

Die Öko-Käfer. Zum Einfluss von Sprache auf die Problemlöse- und Gedächtnisleistung bei der Bearbeitung eines nicht-sprachlichen Problems.

Memorandum No. 31

Bamberg: Otto-Friedrich-Universität 1998

BERNARD, R.:

Early Evaluation of product properties within the Integrated Product Development.

Aachen: Shaker 1999. (Konstruktionstechnik München, Volume 35)

München: TU, Diss. 1999

\section{BERTAU, M.-C.:}

Spuren des Gedächtnisses in innerer Sprache. Versuch einer Analyse der dialogischen Anteile des lauten Denkens.

Sprache \& Kognition 1/2 (1999) pp. 4-19.

BOLTE, A.:

Beim CAD geht das Konstruieren langsamer als das Denken - Zum Einfluß des Einsatzes von CAD-Systemen auf das Arbeitshandeln von Planern.

Arbeit 4 (1998) 7, pp. 362-379. 
COLlins, A. M.; QUILLIAN, M. R.:

Retrieval Time from Semantic Memory.

Journal of Verbal Learning and Verbal Behavior 8 (1969) pp. 240-247.

CONAWAY, J.:

Integrated Product Development: The Technology.

PDM Information Center 1995.

Date of access: 01.01.2003, URL: http://www.pdmic.com/articles/artIPD1.html

CRYSTAL, D.:

Die Cambridge-Enzyklopädie der Sprache.

Frankfurt am Main: Campus 1995.

DÖRNER, D.:

Problemlösen als Informationsverarbeitung. 3rd Edition

Stuttgart: Kohlhammer 1987.

DÖRNER, D.: (1999A)

Bauplan für eine Seele.

Reinbek: Rowohlt 1999.

DÖRNER, D.: (1999B)

Das Denken beim Konstruieren. In: Pahl, G. (Ed.): Prof. Dr.-Ing. E.h. Dr.-Ing. Wolfgang Beitz zum Gedenken - Sein Wirken und Schaffen.

Berlin: Springer 1999, pp. 217-224.

DORSCH, F. (ED.):

Psychologisches Wörterbuch.

Bern: Hans Huber 1982.

DREISTADT, R.:

An Analysis of the use of Analogies and Metaphors in Science.

Journal of Psychology 68 (1968) pp. 97-116.

EHRLENSPIEL, K.; KIEWERT, A.; LINDEMANN, U.:

Kostengünstig Entwickeln und Konstruieren: Kostenmanagement bei der integrierten

Produktentwicklung.

Berlin: Springer 1998.

EHRLENSPIEL, K.:

Integrierte Produktentwicklung. 2nd Edition

München: Hanser 2003.

ENCARNAÇAO, J. L.:

Virtual Environments / Virtual Reality. In: Zilahi-Szabó, M. G. (Ed.): Kleines Lexikon der Informatik und Wirtschaftsinformatik.

München: R. Oldenbourg 1995, pp. 590-594. 
EVERSHEIM, W. ET AL. (ED.):

Schnelle Produktentwicklung - 3D-Modellierung und Rapid Prototyping. In:

Wettbewerbsfaktor Produktionstechnik, Tagungsband des Aachener Werkzeugmaschinen-

Kolloquiums AWK '96, Aachen.

Düsseldorf: VDI-Verlag 1996, pp. 3-3 - 3-41.

FÄHRER, J.:

Ganzheitliche Optimierung des indirekten Metall-Lasersinterprozesses.

München: Herbert Utz 2002.

München: TU, Diss 2002.

FISH, J. C.:

Why Do Designers Sketch?: Visual Cognition and Computer Assisted Visualisation. In: Trappl, R. (Ed.): Proceedings of the Twelth European Meeting on Cybernetics and Systems Research, Vienna, Austria, April 5-8, 1994.

Singapore: World Scientific 1994, pp. 499-506.

Friedmann, S. \& SteVEnson, M.:

Developmental Changes in the Understanding of Implied Motion in Two-dimensional Pictures.

Child Development 46, 1975, pp. 773-778.

Friedmann, S. \& SteVEnSON, M.:

Perception of Movement in Pictures. In: Hagen, M. A. (ed.): The Perception of Pictures.

Volume 1

New York: Academic Press 1980.

GERO, J., TVERSKY, B. (EDS.):

Visual and Spatial Reasoning in Design.

Key Centre of Design Computing and Cognition, University of Sydney: Sydney 1999

GIAPOULIS, A.; SCHLÜTER, A.; EHRLENSPIEL, K.; GÜNTHER, J.:

Effizientes Konstruieren durch generierendes und korrigierendes Vorgehen. In: Hubka, V. et al. (Eds.): Proceedings of ICED 95, Volume 2, Praha.

Zürich: Edition Heurista 1995, pp. 477-483. (Schriftenreihe WDK 23)

GITTLER, B.:

3-D-Würfeltest: Verfahren zur Erfassung des räumlichen Vorstellungsvermögens.

Weilheim: Beltz 1990.

GORDON, W. J.:

Synectics.

New York: Harper \& Row 1961. 


\section{GÜNTHER, J.:}

Individuelle Einflüsse auf den Konstruktionsprozess.

Aachen: Shaker 1998. (Konstruktionstechnik München, Volume 30)

München: TU, Diss. 1998

\section{HACKER, W.:}

Allgemeine Arbeitspsychologie: Psychische Regulation von Arbeitstätigkeiten.

Bern: Hans Huber 1998.

Hacker, W.; SAChSE, P.; WetzStein, A.; Winkelmann, C.:

Action Theory - A Generic Approach to Design Activity. In: Lindemann, U. (Ed.): Human

Behaviour in Design.

Berlin: Springer 2003.

HuSsY, W. (1983):

Komplexe menschliche Informationsverarbeitung: Das SPIV-Modell.

Sprache und Kognition 2(1983), pp. 47-62.

HuSSY, W. (1984A):

Denkpsychologie. Ein Lehrbuch. Vol. 1

Stuttgart: Kohlhammer 1982.

HuSSY, W. (1984B):

Denkpsychologie. Ein Lehrbuch. Vol. 2

Stuttgart: Kohlhammer 1982.

ISO 9241-1:

Ergonomic requirements for office work with visual display terminals (VDTs). Part 1: General introduction.

International Organization for Standardization 1997.

KIM, H.; FRANCIS, G.:

A Computational and Perceptual Account of Motion Lines.

Perception 27, 1998, pp. 785-797.

KOFFKA, K.:

Principles of Gestalt Psychology.

New York: Harcourt-Brace 1935.

Kosslyn, S. M.; PINKER, S.; SMITH, G. E.; SwarTZ, S. P.:

On the demystification of mental imagery.

The Behavioral and Brain Sciences (2), 1979, pp. 535-581.

KOSSLYN, S. M.:

Image and Brain: The Resolution of the Imagery Debate.

Cambridge: MIT Press 1994. 
LEHMANN, E.:

Automatisches Problemlösen. In: Klix, F.; Krause, W.; Sydow, H.: Kybernetik-Forschung. Berlin: 2. Deutscher Verlag der Wissenschaft 1972.

LEONTJEW, A. N.:

Tätigkeit, Bewusstsein, Persönlichkeit.

Berlin: Volk und Wissen 1979.

LEWIN, K.:

Untersuchung zur Handlungs- und Affektpsychologie.

Psychologische Forschung 7 (1926), pp.295-385.

LINDEMANN, U.; KLEEDÖRFER, R.:

Erfolgreiche Produkte durch integrierte Produktentwicklung. In: Reinhard, G.; Milberg, J.

(Eds.): Mit Schwung zum Aufschwung, Münchner Kolloquium '97.

Landberg/Lech: Moderne Industrie 1997, pp. 115-136.

LINDEMANN, U. (2003A):

CAD in der Produktentwicklung. Lecture script.

München: Lehrstuhl für Produktentwicklung 2003.

LINDEMANN, U. (2003B):

Produktentwicklung und Konstruktion. Lecture script.

München: Lehrstuhl für Produktentwicklung 2003.

LINDEMANN, U. (2003C):

Methods are Networks of Methods.

In: Folkeson, A.; Gralén, K.; Norell, M.; Sellgren, U. (Eds.): Proceedings of the 14th International Conference on Engineering Design 2003 (ICED03), Stockholm, 19.-21.08.2003.

Design Society 2003.

LINDEMANN, U.:

Methodische Entwicklung technischer Produkte: Methoden flexible und situationsgerecht anwenden.

Berlin: Springer 2004.

MCCARTHY, J.:

The Inversion of Functions Defined by Turing Machines.

Annals of Mathematical Studies 34 (1956) pp. 177-181.

MERRIAM-WEBSTER:

WWWebster Dictionary 2003.

Date of access: 01.01.2003, URL: http://www.m-w.com 
MilgRAM, P.; KishinO, F.:

A Taxonomy of Mixed Virtual Reality Displays.

IECE Transactions on Information and Systems (Special Issue Networked Reality)

Vol. E77-D (12), pp. 1321-1329.

MILLER, W. R.:

The Definition of Design.

MethodJournal (Internet magazine) Date of access: 01.01.2003,

URL: http://www.methodjournal.com/artman/publish/article_14.shtml

MiLLER, G. A.:

The Magical Number Seven, Plus Minus Two. Some Limits on our Capacity for Processing Information.

Psychological Review 63 (1956) pp. 81-97.

Miller, G. A.; Galanter, E.; PriBram, K. H.:

Plans and the Structure of Behavior.

London: Holt, Rinehart and Winston 1960.

MÜLleR, F.; PACHE, M.; SCHNEIDER, S.; LindeMANN, U.:

Free-Hand Sketching for creative work in early design phases. In: Proceedings of the 1st International Conference on Advanced Research in Virtual and Rapid Prototyping, Leiria, Portugal, 1.-4.10.2003.

Leiria: Escola Superior de Tecnologia e Gestão de Leiria, 2003.

MÜLLER, F:

Digitale Modellierung Unscharfer Geometrien.

München: TU, Diss. 2004 (estimated completion).

Newell, A.; SHAw, J. C.; SimON, H. A.:

Report on a General Problem-Solving Program for a Computer. In: Proceedings of the International Conference on Information Processing, Paris.

Paris: UNESCO 1960, pp. 256-264.

OSBORN, A. F.:

Applied Imagination: Principles and Procedures of Creative Problem-Solving. 3rd Edition New York: Charles Scribner's Sons 1963.

PACHE, M.; LINDEMANN, U.; RÖMER, A.; HACKER, W.:

The Use of Sketches, Physical Models and CAD in the Engineering Design Process. In:

Proceedings of the 5th World Conference on Integrated Design and Process Technology (IDPT 2000), Dallas.

Dallas: Society for Design and Process Science (SDPS) 2000. (CD-ROM) 
PAChe, U.; Weißhahn, G.; RÖMER, A.; Lindemann, U.; HACKer, W.:

Effort-saving Modeling in Early Stages of the Design Process. In: Lindemann, U.; Birkhofer, H.; Meerkamm, H.; Vajna, S. (Eds.): Proceedings of ICED 99, München. Volume 2 München: Technische Universität München 1999, pp. 679-684.

PAHL, G.; BEITZ, W.:

Engineering Design: A Systematic Approach. 2nd Edition

London: Springer 1996.

POINCARÉ, H.:

Die mathematische Erfindung. In: Ulmann, G. (Ed.): Kreativitätsforschung.

Köln: Kiepenheuer \& Witsch 1973.

PYLYSHYN, Z. W.:

What the Mind's Eye Tells the Mind's Brain: A Critique of Mental Imagery.

Psychological Bulletin (80), 1973, pp. 1-24.

RÖMER, A.; PAChe, M.; Weißhahn, G.; Lindemann, U.; HACKer, W.:

Effort-Saving Product Representations in Design - Results of a Questionnaire Survey.

Design Studies 22 (2001) 6, pp. 473-491.

RÖMER, A.:

Unterstützung des Design Problem Solving: Einsatz und Nutzen einfacher externer Hilfsmittel in den frühen Phasen des konstruktiven Entwurfsprozesses.

Dresden: TU, Diss 2002.

RP-NET:

Your Market-Place for Rapid Prototyping / Rapid Tooling.

Date of access: 01.01.2003, URL: http://www.rp-net.de/englisch

SACHSE, P. \& HACKER, W.:

Unterstützung des Denkens und Handelns beim Konstruieren durch Prototyping.

Konstruktion 49 (1997), pp. 12-16.

SCHOEN, D. A.:

The Reflective Practicioner.

New York: Basic Books 1983.

SCHRODA, F.:

Über das Ende wird am Anfang entschieden: Zur Analyse der Anforderungen von

Konstruktionsaufträgen.

Date of access: 01.01.2003, URL: http://edocs.tu-berlin.de/diss/2000/schroda_frauke.htm

Berlin: TU, Diss 2000.

SEUSS, DR.:

The Foot Book.

New York: Random House 1968. 
SHANNON, C. E.; WEAVER, W.:

The Mathematical Theory of Communication.

Illinois: University of Illinois Press 1949.

SPUR, G.; KRAUSE, F.-L.:

Das virtuelle Produkt: Management der CAD-Technik.

München: Hanser 1997.

STACEY, M.; ECKERT, C.:

CAD System Bias in Engineering design. In: Lindemann, U.; Birkhofer, H.; Meerkamm, H.;

Vajna, S. (Eds.): Proceedings of ICED 99, München. Volume 3

München: Technische Universität München 1999, pp. 1413-1418.

STACEY, M.; ECKERT, C.; MCFADZEAN, J.:

Sketch Interpretation in Design Communication. In: Lindemann, U.; Birkhofer, H.; Meerkamm, H.; Vajna, S. (eds.): Proceedings of ICED 99, München. Volume 2

München: Technische Universität München 1999, pp. 923-928. (Schriftenreihe WDK 26)

STÄUDEL, T.:

Der Kompetenzfragebogen.

Diagnostica 34(2) 1988, pp. 136-148.

STETTER, R.; PACHE, M.:

Körperliche Modelle in der Produktentwicklung. In: Milberg, J.; Reinhart, G. (Eds.): Rapid Prototyping. Effizienter Einsatz von Modellen in der Produktentwicklung, München.

München: Herbert Utz 1998.

SuWA, M., Gero, J., PuRCELl, T.:

Macroscopic Analysis of Design Processes Based on a Scheme for Coding Designers' Cognitive Actions.

Design Studies 19 (1998) 4, pp. 455-483.

SuWA, M., GERO, J., PURCELl, T.:

Unexpected discoveries: How designers discover hidden features in sketches. In: Gero, J., Tversky, B. (eds.): Visual and Spatial Reasoning in Design.

Key Centre of Design Computing and Cognition, University of Sydney: Sydney 1999, pp. 145162.

\section{ULLMANN, D.:}

The Mechanical Design Process. 2nd Edition

Mc Graw-Hill 1997.

\section{VDI-NACHRICHTEN}

Auf die Handskizze können Konstrukteure nicht verzichten

VDI-Nachrichten, München 23.03.2001 
VDI-RICHTLINIE 2221:

Methodik zum Entwickeln und Konstruieren technischer Systeme und Produkte.

Düsseldorf: VDI-Verlag 1993.

VON DER WETH, R.:

Management der Komplexität: Ressourcenorientiertes Handeln in der Praxis.

Bern: Huber 2001.

WALLAS, G.:

The Art of Thought.

New York: Harcourt 1926.

WULF, J.:

Elementarmethoden zur Lösungssuche.

München: Dr. Hut 2002. (Produktentwicklung München, Volume 50)

München: TU, Diss. 2002 
This book is available at Verlag Dr. Hut, München, www.dr.hut-verlag.de (ISBN 3-89963-176-5) 


\section{List of Dissertations Lehrstuhl für Produktentwicklung}

Lehrstuhl für Produktentwicklung

Technische Universität München,

Boltzmannstraße 15,

85748 Garching

Dissertations under Supervision of

- $\quad$ Prof. Dr.-Ing. W. Rodenacker,

- $\quad$ Prof. Dr.-Ing. K. Ehrlenspiel and

- $\quad$ Prof. Dr.-Ing. U. Lindemann

D1 COLLIN, H.:

Entwicklung eines Einwalzenkalanders nach einer systematischen Konstruktionsmethode.

München: TU, Diss. 1969.

D2 OTT, J.:

Untersuchungen und Vorrichtungen zum Offen-End-Spinnen.

München: TU, Diss. 1971.

D3 STEINWACHS, H.:

Informationsgewinnung an bandförmigen Produkten für die Konstruktion der Produktmaschine.

München: TU, Diss. 1971.

D4 SCHMETTOW, D.:

Entwicklung eines Rehabilitationsgerätes für Schwerstkörperbehinderte.

München: TU, Diss. 1972.

D5 LUBITZSCH, W.:

Die Entwicklung eines Maschinensystems zur Verarbeitung von chemischen Endlosfasern.

München: TU, Diss. 1974.

D6 SCHEITENBERGER, H.:

Entwurf und Optimierung eines Getriebesystems für einen Rotationsquerschneider mit allgemeingültigen Methoden.

München: TU, Diss. 1974.

D7 BAUMGARTH, R.:

Die Vereinfachung von Geräten zur Konstanthaltung physikalischer Größen.

München: TU, Diss. 1976. 
D8 MAUDERER, E.:

Beitrag zum konstruktionsmethodischen Vorgehen durchgeführt am Beispiel eines

Hochleistungsschalter-Antriebs.

München: TU, Diss. 1976.

D9 SCHÄFER, J.:

Die Anwendung des methodischen Konstruierens auf verfahrenstechnische Aufgabenstellungen.

München: TU, Diss. 1977.

D10 WEBER, J.:

Extruder mit Feststoffpumpe - Ein Beitrag zum Methodischen Konstruieren.

München: TU, Diss. 1978.

D11 HEISIG, R.:

Längencodierer mit Hilfsbewegung.

München: TU, Diss. 1979.

D12 KIEWERT, A.:

Systematische Erarbeitung von Hilfsmitteln zum kostenarmen Konstruieren.

München: TU, Diss. 1979.

D13 LINDEMANN, U.:

Systemtechnische Betrachtung des Konstruktionsprozesses unter besonderer Berücksichtigung

der Herstellkostenbeeinflussung beim Festlegen der Gestalt.

Düsseldorf: VDI-Verlag 1980. (Fortschritt-Berichte der VDI-Zeitschriften Reihe 1, Nr. 60).

Zugl. München: TU, Diss. 1980.

D14 NJOYA, G.:

Untersuchungen zur Kinematik im Wälzlager bei synchron umlaufenden Innen- und

Außenringen.

Hannover: Universität, Diss. 1980.

D15 HENKEL, G.:

Theoretische und experimentelle Untersuchungen ebener konzentrisch gewellter

Kreisringmembranen.

Hannover: Universität, Diss. 1980.

D16 BALKEN, J.:

Systematische Entwicklung von Gleichlaufgelenken.

München: TU, Diss. 1981.

D17 PETRA, H.:

Systematik, Erweiterung und Einschränkung von Lastausgleichslösungen für Standgetriebe mit zwei Leistungswegen - Ein Beitrag zum methodischen Konstruieren.

München: TU, Diss. 1981. 
D18 BAUMANN, G.:

Ein Kosteninformationssystem für die Gestaltungsphase im Betriebsmittelbau.

München: TU, Diss. 1982.

D19 FISCHER, D.:

Kostenanalyse von Stirnzahnrädern. Erarbeitung und Vergleich von Hilfsmitteln zur

Kostenfrüherkennung.

München: TU, Diss. 1983.

D20 AUGUSTIN, W.:

Sicherheitstechnik und Konstruktionsmethodiken - Sicherheitsgerechtes Konstruieren.

Dortmund: Bundesanstalt für Arbeitsschutz 1985.

Zugl. München: TU, Diss. 1984.

D21 RUTZ, A.:

Konstruieren als gedanklicher Prozeß.

München: TU, Diss. 1985.

D22 SAUERMANN, H. J.:

Eine Produktkostenplanung für Unternehmen des Maschinenbaues.

München: TU, Diss. 1986.

D23 HAFNER, J.:

Entscheidungshilfen für das kostengünstige Konstruieren von Schweiß- und Gußgehäusen.

München: TU, Diss. 1987.

D24 JOHN, T.:

Systematische Entwicklung von homokinetischen Wellenkupplungen.

München: TU, Diss. 1987.

D25 FIGEL, K.:

Optimieren beim Konstruieren.

München: Hanser 1988.

Zugl. München: TU, Diss. 1988 u. d. T.: Figel, K.: Integration automatisierter

Optimierungsverfahren in den rechnerunterstützten Konstruktionsprozeß.

\section{Reihe Konstruktionstechnik München}

D26 TROPSCHUH, P. F.:

Rechnerunterstützung für das Projektieren mit Hilfe eines wissensbasierten Systems.

München: Hanser 1989. (Konstruktionstechnik München, Band 1).

Zugl. München: TU, Diss. 1988 u. d. T.: Tropschuh, P. F.: Rechnerunterstützung für das Projektieren am Beispiel Schiffsgetriebe. 


\section{D27 PICKEL, H.:}

Kostenmodelle als Hilfsmittel zum Kostengünstigen Konstruieren. München: Hanser 1989. (Konstruktionstechnik München, Band 2).

Zugl. München: TU, Diss. 1988.

\section{D28 KITTSTEINER, H.-J.:}

Die Auswahl und Gestaltung von kostengünstigen Welle-Nabe-Verbindungen.

München: Hanser 1990. (Konstruktionstechnik München, Band 3).

Zugl. München: TU, Diss. 1989.

D29 HILLEBRAND, A.:

Ein Kosteninformationssystem für die Neukonstruktion mit der Möglichkeit zum Anschluß an ein CAD-System.

München: Hanser 1991. (Konstruktionstechnik München, Band 4).

Zugl. München: TU, Diss. 1990.

\section{D30 DYLLA, N.:}

Denk- und Handlungsabläufe beim Konstruieren.

München: Hanser 1991. (Konstruktionstechnik München, Band 5).

Zugl. München: TU, Diss. 1990.

\section{D31 MÜLLER, R.}

Datenbankgestützte Teileverwaltung und Wiederholteilsuche.

München: Hanser 1991. (Konstruktionstechnik München, Band 6).

Zugl. München: TU, Diss. 1990.

\section{D32 NEESE, J.:}

Methodik einer wissensbasierten Schadenanalyse am Beispiel Wälzlagerungen.

München: Hanser 1991. (Konstruktionstechnik München, Band 7).

Zugl. München: TU, Diss. 1991.

D33 SCHAAL, S.:

Integrierte Wissensverarbeitung mit CAD - Am Beispiel der konstruktionsbegleitenden

Kalkulation.

München: Hanser 1992. (Konstruktionstechnik München, Band 8).

Zugl. München: TU, Diss. 1991.

\section{D34 BRAUNSPERGER, M.:}

Qualitätssicherung im Entwicklungsablauf - Konzept einer präventiven Qualitätssicherung für die Automobilindustrie.

München: Hanser 1993. (Konstruktionstechnik München, Band 9).

Zugl. München: TU, Diss. 1992. 
D35 FEICHTER, E.:

Systematischer Entwicklungsprozeß am Beispiel von elastischen Radialversatzkupplungen. München: Hanser 1994. (Konstruktionstechnik München, Band 10).

Zugl. München: TU, Diss. 1992.

D36 WEINBRENNER, V.:

Produktlogik als Hilfsmittel zum Automatisieren von Varianten $\square$ und Anpassungskonstruktionen.

München: Hanser 1994. (Konstruktionstechnik München, Band 11).

Zugl. München: TU, Diss. 1993.

D37 WACH, J. J.:

Problemspezifische Hilfsmittel für die Integrierte Produktentwicklung. München: Hanser 1994. (Konstruktionstechnik München, Band 12).

Zugl. München: TU, Diss. 1993.

D38 LENK, E.:

Zur Problematik der technischen Bewertung.

München: Hanser 1994. (Konstruktionstechnik München, Band 13).

Zugl. München: TU, Diss. 1993.

D39 STUFFER, R.:

Planung und Steuerung der Integrierten Produktentwicklung.

München: Hanser 1994. (Konstruktionstechnik München, Band 14).

Zugl. München: TU, Diss. 1993.

D40 SCHIEBELER, R.:

Kostengünstig Konstruieren mit einer rechnergestützten Konstruktionsberatung.

München: Hanser 1994. (Konstruktionstechnik München, Band 15).

Zugl. München: TU, Diss. 1993.

\section{D41 BRUCKNER, J.:}

Kostengünstige Wärmebehandlung durch Entscheidungsunterstützung in Konstruktion und Härterei.

München: Hanser 1994. (Konstruktionstechnik München, Band 16).

Zugl. München: TU, Diss. 1993.

D42 WELLNIAK, R.:

Das Produktmodell im rechnerintegrierten Konstruktionsarbeitsplatz. München: Hanser 1994. (Konstruktionstechnik München, Band 17).

Zugl. München: TU, Diss. 1994.

D43 SCHLÜTER, A.:

Gestaltung von Schnappverbindungen für montagegerechte Produkte. München: Hanser 1994. (Konstruktionstechnik München, Band 18).

Zugl. München: TU, Diss. 1994. 


\section{D44 WOLFRAM, M.:}

Feature-basiertes Konstruieren und Kalkulieren.

München: Hanser 1994. (Konstruktionstechnik München, Band 19).

Zugl. München: TU, Diss. 1994.

\section{D45 STOLZ, P.:}

Aufbau technischer Informationssysteme in Konstruktion und Entwicklung am Beispiel eines elektronischen Zeichnungsarchives.

München: Hanser 1994. (Konstruktionstechnik München, Band 20).

Zugl. München: TU, Diss. 1994.

\section{D46 STOLL, G.:}

Montagegerechte Produkte mit feature-basiertem CAD.

München: Hanser 1994. (Konstruktionstechnik München, Band 21).

Zugl. München: TU, Diss. 1994.

\section{D47 STEINER, J. M.:}

Rechnergestütztes Kostensenken im praktischen Einsatz.

Aachen: Shaker 1996. (Konstruktionstechnik München, Band 22).

Zugl. München: TU, Diss. 1995.

\section{D48 HUBER, T.:}

Senken von Montagezeiten und -kosten im Getriebebau.

München: Hanser 1995. (Konstruktionstechnik München, Band 23).

Zugl. München: TU, Diss. 1995.

D49 DANNER, S.:

Ganzheitliches Anforderungsmanagement für marktorientierte Entwicklungsprozesse.

Aachen: Shaker 1996. (Konstruktionstechnik München, Band 24).

Zugl. München: TU, Diss. 1996.

\section{D50 MERAT, P.:}

Rechnergestützte Auftragsabwicklung an einem Praxisbeispiel.

Aachen: Shaker 1996. (Konstruktionstechnik München, Band 25).

Zugl. München: TU, Diss. 1996 u. d. T.: MERAT, P.: Rechnergestütztes Produktleitsystem

\section{D51 AMBROSY, S.:}

Methoden und Werkzeuge für die integrierte Produktentwicklung.

Aachen: Shaker 1997. (Konstruktionstechnik München, Band 26).

Zugl. München: TU, Diss. 1996.

\section{D52 GIAPOULIS, A.:}

Modelle für effektive Konstruktionsprozesse.

Aachen: Shaker 1998. (Konstruktionstechnik München, Band 27).

Zugl. München: TU, Diss. 1996. 


\section{D53 STEINMEIER, E.:}

Realisierung eines systemtechnischen Produktmodells - Einsatz in der Pkw-Entwicklung.

Aachen: Shaker 1998. (Konstruktionstechnik München, Band 28).

Zugl. München: TU, Diss. 1998.

\section{D54 KLEEDÖRFER, R.:}

Prozeß- und Änderungsmanagement der Integrierten Produktentwicklung.

Aachen: Shaker 1998. (Konstruktionstechnik München, Band 29).

Zugl. München: TU, Diss. 1998.

\section{D55 GÜNTHER, J.:}

Individuelle Einflüsse auf den Konstruktionsprozeß.

Aachen: Shaker 1998. (Konstruktionstechnik München, Band 30).

Zugl. München: TU, Diss. 1998.

\section{D56 BIERSACK, H.:}

Methode für Kraftleinleitungsstellenkonstruktion in Blechstrukturen.

München: TU, Diss. 1998.

\section{D57 IRLINGER, R.:}

Methoden und Werkzeuge zur nachvollziehbaren Dokumentation in der Produktentwicklung. Aachen: Shaker 1998. (Konstruktionstechnik München, Band 31).

Zugl. München: TU, Diss. 1999.

\section{D58 EILETZ, R.:}

Zielkonfliktmanagement bei der Entwicklung komplexer Produkte - am Bsp. PKW-

Entwicklung.

Aachen: Shaker 1999. (Konstruktionstechnik München, Band 32).

Zugl. München: TU, Diss. 1999.

D59 STÖSSER, R.:

Zielkostenmanagement in integrierten Produkterstellungsprozessen.

Aachen: Shaker 1999. (Konstruktionstechnik München, Band 33).

Zugl. München: TU, Diss. 1999.

D60 PHLEPS, U.:

Recyclinggerechte Produktdefinition - Methodische Unterstützung für Upgrading und Verwertung.

Aachen: Shaker 1999. (Konstruktionstechnik München, Band 34).

Zugl. München: TU, Diss. 1999.

D61 BERNARD, R.:

Early Evaluation of Product Properties within the Integrated Product Development. Aachen: Shaker 1999. (Konstruktionstechnik München, Band 35).

Zugl. München: TU, Diss. 1999. 
D62 ZANKER, W.:

Situative Anpassung und Neukombination von Entwicklungsmethoden.

Aachen: Shaker 1999. (Konstruktionstechnik München, Band 36).

Zugl. München: TU, Diss. 1999.

\section{Reihe Produktentwicklung München}

D63 ALLMANSBERGER, G.:

Erweiterung der Konstruktionsmethodik zur Unterstützung von Änderungsprozessen in der

Produktentwicklung.

München: Dr. Hut 2001. (Produktentwicklung München, Band 37).

Zugl. München: TU, Diss. 2000.

\section{D64 ASSMANN, G.:}

Gestaltung von Änderungsprozessen in der Produktentwicklung.

München: Utz 2000. (Produktentwicklung München, Band 38).

Zugl. München: TU, Diss. 2000.

\section{D65 BICHLMAIER, C.:}

Methoden zur flexiblen Gestaltung von integrierten Entwicklungsprozessen.

München: Utz 2000. (Produktentwicklung München, Band 39).

Zugl. München: TU, Diss. 2000.

\section{D66 DEMERS, M. T.}

Methoden zur dynamischen Planung und Steuerung von Produktentwicklungsprozessen.

München: Dr. Hut 2000. (Produktentwicklung München, Band 40).

Zugl. München: TU, Diss. 2000.

\section{D67 STETTER, R.:}

Method Implementation in Integrated Product Development.

München: Dr. Hut 2000. (Produktentwicklung München, Band 41).

Zugl. München: TU, Diss. 2000.

\section{D68 VIERTLBÖCK, M.:}

Modell der Methoden- und Hilfsmitteleinführung im Bereich der Produktentwicklung.

München: Dr. Hut 2000. (Produktentwicklung München, Band 42).

Zugl. München: TU, Diss. 2000.

D69 COLLIN, H.:

Management von Produkt-Informationen in kleinen und mittelständischen Unternehmen.

München: Dr. Hut 2001. (Produktentwicklung München, Band 43).

Zugl. München: TU, Diss. 2001. 
D70 REISCHL, C.:

Simulation von Produktkosten in der Entwicklungsphase.

München: Dr. Hut 2001. (Produktentwicklung München, Band 44).

Zugl. München: TU, Diss. 2001.

D71 GAUL, H.-D.:

Verteilte Produktentwicklung - Perspektiven und Modell zur Optimierung.

München: Dr. Hut 2001. (Produktentwicklung München, Band 45).

Zugl. München: TU, Diss. 2001.

D72 GIERHARDT, H.:

Global verteilte Produktentwicklungsprojekte - Ein Vorgehensmodell auf der operativen Ebene.

München: Dr. Hut 2002. (Produktentwicklung München, Band 46).

Zugl. München: TU, Diss. 2001.

D73 SCHOEN, S.:

Gestaltung und Unterstützung von Community of Practice.

München: Utz 2000. (Produktentwicklung München, Band 47).

Zugl. München: TU, Diss. 2000.

D74 BENDER, B.:

Zielorientiertes Kooperationsmanagement.

München: Dr. Hut 2001. (Produktentwicklung München, Band 48).

Zugl. München: TU, Diss. 2001.

D75 SCHWANKL, L.:

Analyse und Dokumentation in den frühen Phasen der Produktentwicklung.

München: Dr. Hut 2002. (Produktentwicklung München, Band 49).

Zugl. München: TU, Diss. 2002.

D76 WULF, J.:

Elementarmethoden zur Lösungssuche.

München: Dr. Hut 2002. (Produktentwicklung München, Band 50).

Zugl. München: TU, Diss. 2002.

D77 MÖRTL, M.:

Entwicklungsmanagement für langlebige, upgradinggerechte Produkte.

München: Dr. Hut 2002. (Produktentwicklung München, Band 51).

Zugl. München: TU, Diss. 2002.

D78 GERST, M.:

Strategische Produktentscheidungen in der integrierten Produktentwicklung.

München: Dr. Hut 2002. (Produktentwicklung München, Band 52).

Zugl. München: TU, Diss. 2002. 
D79 AMFT, M.:

Phasenübergreifende bidirektionale Integration von Gestaltung und Berechnung.

München: Dr. Hut 2003. (Produktentwicklung München, Band 53).

Zugl. München: TU, Diss. 2002.

D80 FÖRSTER, M.:

Variantenmanagement nach Fusionen in Unternehmen des Anlagen- und Maschinenbaus.

München: Dr. Hut 2003. (Produktentwicklung München, Band 54).

Zugl. München: TU, Diss. 2003.

\section{D81 GRAMANN, J.:}

Problemmodelle und Bionik als Methode.

München: Dr. Hut 2004. (Produktentwicklung München, Band 55).

Zugl. München: TU, Diss. 2004.

\section{D82 PULM, U.:}

Eine systemtheoretische Betrachtung der Produktentwicklung.

München: Dr. Hut 2004. (Produktentwicklung München, Band 56).

Zugl. München: TU, Diss. 2004. 